Supporting Information for

\title{
Total Synthesis and Structural Revision of
}

\section{Greensporone F and Dechlorogreensporone F}

Janardhan Gaddam, ${ }^{\dagger, \S, \neq}$ Aedula Vishnu V. Reddy, ${ }^{\dagger, \S, \neq}$ AkellaV. S. Sarma, ${ }^{\ddagger}$ Jhillu S. Yadav ${ }^{\dagger, \S, ! !}$ and Debendra K. Mohapatra*,†,

${ }^{\dagger}$ Department of Organic Synthesis and Process Chemistry and ${ }^{\ddagger}$ Centre for NMR and Structural Chemistry, CSIR-Indian Institute of Chemical Technology, Hyderabad-500007, India; ${ }^{\S}$ Academy of Scientific and Innovative Research (AcSIR), Ghaziabad, Uttar Pradesh-201002, India, "School of Science, Indrashil University, Kadi, Gujarat, India. 
Table of Contents

\begin{tabular}{|c|c|c|}
\hline S. No & Description & Page No. \\
\hline 1 & Mosher's analysis of compound $\mathbf{1 8}$ and $\mathbf{1 8 a}$ & S06 \\
\hline 2 & $\begin{array}{l}\text { Table S1. Comparison of }{ }^{1} \mathrm{H} \text { and }{ }^{13} \mathrm{C} \text { chemical shifts } \\
\text { and } \mathrm{H}-\mathrm{H} \text { scalar couplings of the proposed structure } \\
\text { of Dechlorogreensporone } \mathrm{F} \text { with } \\
\text { the synthetic compound } \mathbf{4 a}\end{array}$ & S07 \\
\hline 3 & $\begin{array}{l}\text { Table S2. Comparison of }{ }^{1} \mathrm{H} \text { and }{ }^{13} \mathrm{C} \text { chemical shifts } \\
\text { and } \mathrm{H}-\mathrm{H} \text { scalar couplings of the proposed structure } \\
\text { of Greensporone } \mathrm{F} \text { with the synthetic compound } \mathbf{5 a}\end{array}$ & S8 \\
\hline 4 & $\begin{array}{l}\text { Table S3. Comparison of }{ }^{1} \mathrm{H} \text { and }{ }^{13} \mathrm{C} \text { chemical shifts } \\
\text { and } \mathrm{H}-\mathrm{H} \text { scalar couplings of the proposed structure } \\
\text { of Dechlorogreensporone } \mathrm{F} \text { with } \\
\text { the synthetic compound } \mathbf{4}\end{array}$ & S9 \\
\hline 5 & $\begin{array}{l}\text { Table S4. Comparison of }{ }^{1} \mathrm{H} \text { and }{ }^{13} \mathrm{C} \text { chemical shifts } \\
\text { and } \mathrm{H}-\mathrm{H} \text { scalar couplings of the proposed structure } \\
\text { of Greensporone } \mathrm{F} \text { with the synthetic compound } 5\end{array}$ & S10 \\
\hline 6 & ${ }^{1} \mathrm{H}$ and ${ }^{13} \mathrm{C}$ NMR Copy of $\mathbf{1 5}$ & $\mathrm{S} 11-\mathrm{S} 12$ \\
\hline 7 & ${ }^{1} \mathrm{H}$ and ${ }^{13} \mathrm{C}$ NMR Copy of $\mathbf{1 6}$ & S13-S14 \\
\hline 8 & ${ }^{1} \mathrm{H}$ and ${ }^{13} \mathrm{C}$ NMR Copy of $\mathbf{1 7}$ & S15-S16 \\
\hline 9 & ${ }^{1} \mathrm{H}$ and ${ }^{13} \mathrm{C}$ NMR Copy of $\mathbf{1 8}$ & S17-S18 \\
\hline 10 & ${ }^{1} \mathrm{H}$ NMR Copy of $\mathbf{1 8}$ & S19 \\
\hline 11 & ${ }^{1} \mathrm{H}$ NMR Copy of 18" & S20 \\
\hline 12 & ${ }^{1} \mathrm{H}$ and ${ }^{13} \mathrm{C}$ NMR Copy of 19 & $\mathrm{~S} 21-\mathrm{S} 22$ \\
\hline 13 & ${ }^{1} \mathrm{H}$ and ${ }^{13} \mathrm{C}$ NMR Copy of $\mathbf{1 1}$ & S-23-S24 \\
\hline
\end{tabular}




\begin{tabular}{|c|c|c|}
\hline 14 & ${ }^{1} \mathrm{H}$ and ${ }^{13} \mathrm{C}$ NMR Copy of 20a & $\mathrm{S} 25-\mathrm{S} 26$ \\
\hline 15 & ${ }^{1} \mathrm{H}$ and ${ }^{13} \mathrm{C}$ NMR Copy of $\mathbf{2 0 b}$ & $\mathrm{S} 27-\mathrm{S} 28$ \\
\hline 16 & ${ }^{1} \mathrm{H}$ and ${ }^{13} \mathrm{C}$ NMR Copy of 20c & $\mathrm{S} 29-\mathrm{S} 30$ \\
\hline 17 & ${ }^{1} \mathrm{H}$ and ${ }^{13} \mathrm{C}$ NMR Copy of $\mathbf{1 2}$ & S31-S32 \\
\hline 18 & ${ }^{1} \mathrm{H}$ and ${ }^{13} \mathrm{C}$ NMR Copy of $\mathbf{2 1}$ & S33-S34 \\
\hline 19 & ${ }^{1} \mathrm{H}$ and ${ }^{13} \mathrm{C}$ NMR Copy of $\mathbf{1 0}$ & S35-S36 \\
\hline 20 & ${ }^{1} \mathrm{H}$ and ${ }^{13} \mathrm{C}$ NMR Copy of $\mathbf{2 2}$ & S37-S38 \\
\hline 21 & ${ }^{1} \mathrm{H}$ and ${ }^{13} \mathrm{C}$ NMR Copy of $\mathbf{2 3}$ & S39-S40 \\
\hline 22 & ${ }^{1} \mathrm{H}$ and ${ }^{13} \mathrm{C}$ NMR Copy of $\mathbf{2 4}$ & $\mathrm{S} 41-\mathrm{S} 42$ \\
\hline 23 & ${ }^{1} \mathrm{H}$ and ${ }^{13} \mathrm{C}$ NMR Copy of 9 & S42-S44 \\
\hline 24 & ${ }^{1} \mathrm{H}$ and ${ }^{13} \mathrm{C}$ NMR Copy of 7 & S45-S46 \\
\hline 25 & ${ }^{1} \mathrm{H}$ and ${ }^{13} \mathrm{C}$ NMR Copy of $\mathbf{4 a}$ & S47-S48 \\
\hline 26 & ${ }^{1} \mathrm{H}$ and ${ }^{13} \mathrm{C}$ NMR Copy of 8 & $\mathrm{~S} 49-\mathrm{S} 50$ \\
\hline 27 & ${ }^{1} \mathrm{H}$ and ${ }^{13} \mathrm{C}$ NMR Copy of $\mathbf{5 a}$ & S51-S52 \\
\hline 28 & DQF-COSY spectrum of compound $\mathbf{5 a}$ & S53-S56 \\
\hline 29 & ${ }^{13} \mathrm{C}-{ }^{1} \mathrm{H}$ HMBC spectrum of compound $\mathbf{5 a}$ & S57-S59 \\
\hline 30 & HSQC spectrum of compound $\mathbf{5 a}$ & S60-S62 \\
\hline 31 & NOESY spectrum of compound $\mathbf{5 a}$ & S63-S66 \\
\hline
\end{tabular}




\begin{tabular}{|c|c|c|}
\hline 32 & DQF-COSY spectrum of compound $\mathbf{4 a}$ & S67-S68 \\
\hline 33 & NOESY spectrum of compound $\mathbf{4 a}$ & S69-S70 \\
\hline 34 & ${ }^{1} \mathrm{H}$ and ${ }^{13} \mathrm{C}$ NMR Copy of $\mathbf{1 7 a}$ & S71-S72 \\
\hline 35 & ${ }^{1} \mathrm{H}$ and ${ }^{13} \mathrm{C}$ NMR Copy of $\mathbf{1 8 a}$ & S73-S74 \\
\hline 36 & ${ }^{1} \mathrm{H}$ NMR Copy of $\mathbf{1 8} \mathbf{a}^{\prime}$ & S75-S76 \\
\hline 37 & ${ }^{1} \mathrm{H}$ NMR Copy of $\mathbf{1 8 a}$ & S77-S78 \\
\hline 38 & ${ }^{1} \mathrm{H}$ and ${ }^{13} \mathrm{C}$ NMR Copy of $\mathbf{1 9 a}$ & S79 \\
\hline 39 & ${ }^{1} \mathrm{H}$ and ${ }^{13} \mathrm{C}$ NMR Copy of $\mathbf{1 1 a}$ & $\mathrm{S} 80$ \\
\hline 40 & ${ }^{1} \mathrm{H}$ and ${ }^{13} \mathrm{C}$ NMR Copy of $\mathbf{2 2 a}$ & S81-S82 \\
\hline 41 & ${ }^{1} \mathrm{H}$ and ${ }^{13} \mathrm{C}$ NMR Copy of $\mathbf{2 3 a}$ & S83-S84 \\
\hline 42 & ${ }^{1} \mathrm{H}$ and ${ }^{13} \mathrm{C}$ NMR Copy of $\mathbf{2 4 a}$ & S85-S86 \\
\hline 43 & ${ }^{1} \mathrm{H}$ and ${ }^{13} \mathrm{C}$ NMR Copy of $\mathbf{9 a}$ & S87-S88 \\
\hline 44 & ${ }^{1} \mathrm{H}$ and ${ }^{13} \mathrm{C}$ NMR Copy of $7 \mathbf{a}$ & S89-S90 \\
\hline 45 & ${ }^{1} \mathrm{H}$ and ${ }^{13} \mathrm{C}$ NMR Copy of 4 & S91-S92 \\
\hline 46 & ${ }^{1} \mathrm{H}$ and ${ }^{13} \mathrm{C}$ NMR Copy of $\mathbf{8 a}$ & S93-S94 \\
\hline 47 & ${ }^{1} \mathrm{H}$ and ${ }^{13} \mathrm{C}$ NMR Copy of 5 & S95-S96 \\
\hline 48 & DQF-COSY spectrum of compound $\mathbf{5}$ & S97-S100 \\
\hline 49 & ${ }^{13} \mathrm{C}-{ }^{1} \mathrm{H}$ HMBC spectrum of compound 5 & S101-S103 \\
\hline
\end{tabular}




\begin{tabular}{|c|c|c|}
\hline 50 & HSQC spectrum of compound 5 & S104-S106 \\
\hline 51 & NOESY spectrum of compound $\mathbf{5}$ & S107-S111 \\
\hline 52 & DQF-COSY spectrum of compound $\mathbf{4}$ & S112-S114 \\
\hline 53 & ${ }^{13} \mathrm{C}^{1}{ }^{1} \mathrm{H}$ HMBC spectrum of compound $\mathbf{4}$ & $\mathrm{S} 115-\mathrm{S} 118$ \\
\hline 54 & HSQC spectrum of compound $\mathbf{4}$ & $\mathrm{S} 119-\mathrm{S} 121$ \\
\hline 55 & NOESY spectrum of compound $\mathbf{4}$ & $\mathrm{S} 122-\mathrm{S} 126$ \\
\hline
\end{tabular}




\section{Mosher's Analysis:}

Esterification of the alcohol compound $\mathbf{1 8}$ with both $(S)$ - and $(R)$-methoxy(trifluoromethyl)phenylacetic acid (MTPA) showed negative chemical shift difference $\left[\Delta \delta=\left(\delta_{S}-\delta_{R}\right)\right.$ $\times 10^{3}$ ] for protons on $\mathrm{C} 1$ through $\mathrm{C} 4$ (Figure $\mathrm{S} 1$ ), while protons on $\mathrm{C} 9$ through $\mathrm{C} 6$ showed positive chemical shift differences, which is indicative of C5 bearing an $S$-configuration. Therefore, the absolute configuration of C5 was assigned as ' $S$ ' in compound $\mathbf{1 8 .}$

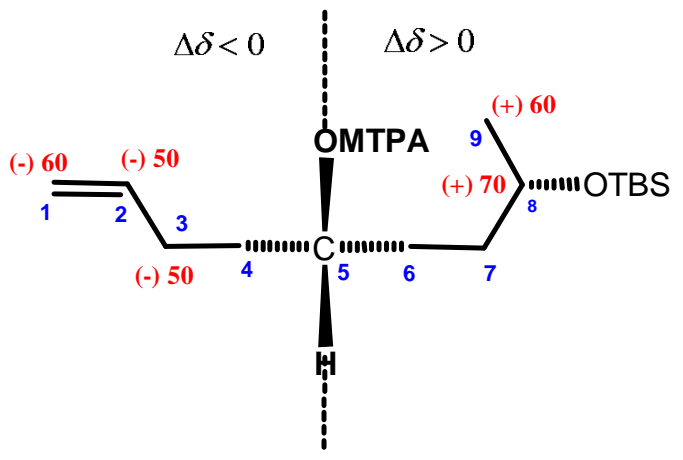

Figure S1. $(\Delta \delta)=\left(\delta_{S}-\delta_{R}\right) \times 10^{3}$ for $(S)$ - and $(R)$-MTPA ester of alcohol compound 18

Esterification of the alcohol compound 18a with both $(S)$ - and $(R)$-methoxy(trifluoromethyl)phenylacetic acid (MTPA) showed negative chemical shift difference $\left[\Delta \delta=\left(\delta_{S}-\delta_{R}\right)\right.$ $\times 10^{3}$ ] for protons on C9 through C6 (Figure S1), while protons on C1 through C4 showed positive chemical shift differences, which is indicative of C5 bearing an $R$-configuration. Therefore, the absolute configuration of $\mathrm{C} 5$ was assigned as ' $R$ ' in compound $\mathbf{1 8 a}$.

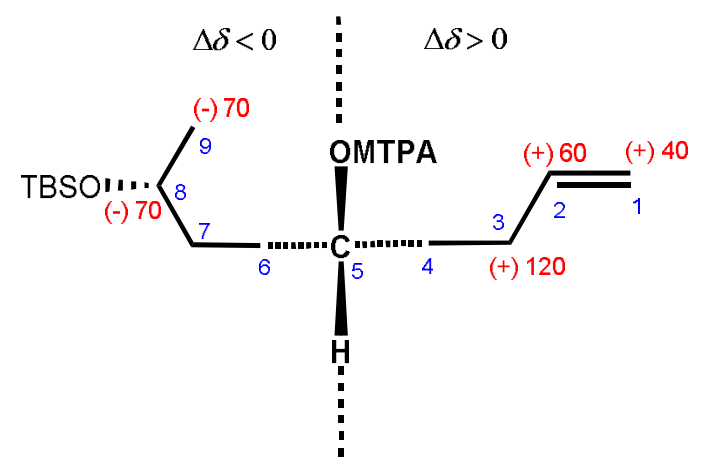

Figure S2. $(\Delta \delta)=\left(\delta_{S}-\delta_{R}\right) \times 10^{3}$ for $(S)$ - and $(R)$-MTPA ester of alcohol compound 18a. 
Table S1. Comparison of ${ }^{1} \mathrm{H}$ and ${ }^{13} \mathrm{C}$ NMR data of Dechlorogreensporone $\mathrm{F}$ with the Synthetic $4 a$

\begin{tabular}{|c|c|c|c|c|}
\hline Position & $\begin{array}{c}{ }^{1} \mathrm{HNMR} \\
\text { of } \\
\text { Dechlorogreensporone } \\
\text { F in ppm } \\
\text { (multiplicity, } J \text { in } \mathrm{Hz} \text { ) }\end{array}$ & $\begin{array}{c}{ }^{1} \text { H NMR } \\
\text { Synthetic Product } \\
\mathbf{4 a} \\
\text { in ppm } \\
\text { (multiplicity, } J \text { in } \\
\text { Hz) }\end{array}$ & $\begin{array}{c}{ }^{13} \mathrm{C} \text { NMR of } \\
\text { Dechlorogreensporone } \\
\text { F } \\
\text { in ppm }\end{array}$ & $\begin{array}{c}{ }^{13} \mathrm{C} \text { NMR } \\
\text { Synthetic Product } \\
\mathbf{4 a} \\
\text { in ppm }\end{array}$ \\
\hline 1 & $1.32(\mathrm{~d}, 6.3)$ & $1.32(\mathrm{~d}, 6.4)$ & 20.9 & 20.8 \\
\hline 2 & $5.26(\mathrm{~m})$ & $5.27(\mathrm{~m})$ & 72.7 & 72.6 \\
\hline 3 & $1.83(\mathrm{~m})$ & $1.84(\mathrm{~m})$ & 33.0 & 32.9 \\
\hline 4 & $\begin{array}{l}1.51(\mathrm{~m}) \\
1.96(\mathrm{~m})\end{array}$ & $\begin{array}{l}1.54(\mathrm{~m}) \\
1.98(\mathrm{~m})\end{array}$ & 31.3 & 31.3 \\
\hline 5 & $3.81(\mathrm{~m})$ & $3.82(\mathrm{~m})$ & 79.5 & 79.4 \\
\hline 6 & $1.50(\mathrm{~m})$ & $1.50(\mathrm{~m})$ & 33.5 & 33.3 \\
\hline 7 & $\begin{array}{l}1.65(\mathrm{~m}) \\
1.94(\mathrm{~m})\end{array}$ & $\begin{array}{l}1.65(\mathrm{~m}) \\
1.93(\mathrm{~m})\end{array}$ & 30.5 & 30.4 \\
\hline 8 & $4.14(\mathrm{~m})$ & $4.13(\mathrm{~m})$ & 76.1 & 76.1 \\
\hline 9 & $\begin{array}{l}2.55(\mathrm{dd}, 13.2,8.0) \\
2.62(\mathrm{dd}, 13.2,3.4)\end{array}$ & $\begin{array}{l}2.55(\mathrm{dd}, 13.7,8.3) \\
2.64(\mathrm{dd}, 13.7,3.6)\end{array}$ & 47.9 & 47.9 \\
\hline 10 & & & 207.7 & 207.7 \\
\hline 11 & $\begin{array}{l}3.90(\mathrm{~d}, 17.2) \\
3.99(\mathrm{~d}, 17.2)\end{array}$ & $\begin{array}{l}3.91(\mathrm{~d}, 16.9) \\
4.02(\mathrm{~d}, 16.9)\end{array}$ & 49.0 & 48.8 \\
\hline 12 & & & 134.2 & 134.1 \\
\hline 13 & $6.25(\mathrm{~d}, 2.3)$ & $6.25(\mathrm{~d}, 1.8)$ & 109.2 & 109.2 \\
\hline 14 & $5.62($ br s) $(\mathrm{OH})$ & $5.90($ br s $)(\mathrm{OH})$ & 157.7 & 157.7 \\
\hline 15 & $6.34(\mathrm{~d}, 2.3)$ & $6.33(\mathrm{~d}, 1.8)$ & 98.3 & 98.3 \\
\hline 16 & & & 159.0 & 159.0 \\
\hline 17 & & & 117.3 & 117.2 \\
\hline 18 & & & 167.7 & 167.6 \\
\hline 19 & $3.77(\mathrm{~s})$ & $3.77(\mathrm{~s})$ & 56.0 & 55.9 \\
\hline
\end{tabular}


Table S2. Comparison of ${ }^{1} \mathrm{H}$ and ${ }^{13} \mathrm{C}$ NMR data of Greensporone $\mathrm{F}$ with the synthetic 5a

\begin{tabular}{|c|c|c|c|c|}
\hline Position & $\begin{array}{c}{ }^{1} \mathrm{H} \text { NMR } \\
\text { of Greensporone } \mathrm{F} \\
\text { in ppm (multiplicity, } \\
J \text { in Hz) }\end{array}$ & $\begin{array}{l}{ }^{1} \mathrm{H} \text { NMR } \\
\text { Synthetic Product 5a } \\
\text { in ppm (multiplicity, } \\
\quad J \text { in Hz) }\end{array}$ & $\begin{array}{c}{ }^{13} \mathrm{C} \text { NMR } \\
\text { of } \\
\text { Greensporone } \mathrm{F} \\
\text { in ppm }\end{array}$ & $\begin{array}{c}{ }^{13} \mathrm{C} \text { NMR } \\
\text { Synthetic Product } \\
\mathbf{5 a} \\
\text { in ppm } \\
\end{array}$ \\
\hline 1 & $1.32(\mathrm{~d}, 6.2)$ & $1.32(\mathrm{~d}, 6.4)$ & 21.5 & 21.5 \\
\hline 2 & $5.21(\mathrm{~m})$ & $5.23(\mathrm{~m})$ & 73.4 & 73.4 \\
\hline 3 & $\begin{array}{l}1.72(\mathrm{~m}) \\
1.90(\mathrm{~m})\end{array}$ & $\begin{array}{l}1.71(\mathrm{~m}) \\
1.90(\mathrm{~m})\end{array}$ & 33.8 & 33.8 \\
\hline 4 & $\begin{array}{l}1.38(\mathrm{~m}) \\
1.79(\mathrm{~m}) \\
\end{array}$ & $\begin{array}{l}1.39(\mathrm{~m}) \\
1.80(\mathrm{~m})\end{array}$ & 34.7 & 34.7 \\
\hline 5 & $3.79(\mathrm{~m})$ & $3.81(\mathrm{~m})$ & 79.3 & 79.3 \\
\hline 6 & $\begin{array}{l}1.55(\mathrm{~m}) \\
2.02(\mathrm{~m})\end{array}$ & $\begin{array}{l}1.55(\mathrm{~m}) \\
2.02(\mathrm{~m})\end{array}$ & 31.6 & 31.5 \\
\hline 7 & $\begin{array}{l}1.75(\mathrm{~m}) \\
1.91(\mathrm{~m}) \\
\end{array}$ & $\begin{array}{l}1.75(\mathrm{~m}) \\
1.91(\mathrm{~m}) \\
\end{array}$ & 29.8 & 29.8 \\
\hline 8 & $4.33(\mathrm{~m})$ & $4.35(\mathrm{~m})$ & 76.3 & 76.3 \\
\hline 9 & $\begin{array}{l}2.53(\mathrm{dd}, 13.5,6.9) \\
2.81(\mathrm{dd}, 13.5,4.6)\end{array}$ & $\begin{array}{l}2.55(\mathrm{dd}, 13.5,7.0) \\
2.81(\mathrm{dd}, 13.4,4.6)\end{array}$ & 46.6 & 46.6 \\
\hline 10 & & & 203.9 & 204.0 \\
\hline 11 & $\begin{array}{l}3.99(\mathrm{~d}, 18.5) \\
4.13(\mathrm{~d}, 18.5) \\
\end{array}$ & $\begin{array}{c}4.0(\mathrm{~d}, 18.4) \\
4.13(\mathrm{~d}, 18.5)\end{array}$ & 48.6 & 48.6 \\
\hline 12 & & & 132.1 & 132.0 \\
\hline 13 & & & 113.1 & 113.1 \\
\hline 14 & $5.70(\mathrm{~s})(\mathrm{OH})$ & $5.87(\mathrm{br} \mathrm{s})(\mathrm{OH})$ & 153.3 & 153.3 \\
\hline 15 & $6.59(\mathrm{~s})$ & $6.57(\mathrm{~s})$ & 99.1 & 99.1 \\
\hline 16 & & & 157.0 & 157.0 \\
\hline 17 & & & 119.2 & 119.1 \\
\hline 18 & & & 166.9 & 166.9 \\
\hline 19 & $3.77(\mathrm{~s})$ & $3.78(\mathrm{~s})$ & 56.4 & 56.3 \\
\hline
\end{tabular}


Table S3. Comparison of ${ }^{1} \mathrm{H}$ and ${ }^{13} \mathrm{C}$ NMR data of Dechlorogreensporone $\mathrm{F}$ with the Synthetic 4

\begin{tabular}{|c|c|c|c|c|}
\hline Position & $\begin{array}{c}{ }^{1} \mathrm{HNMR} \text { data } \\
\text { of Dechloro- } \\
\text { greensporone } \mathrm{F} \text { in ppm } \\
\text { (multiplicity, } J \text { in } \mathrm{Hz} \text { ) }\end{array}$ & $\begin{array}{l}{ }^{1} \text { HNMR data } \\
\text { Synthetic Product } \\
\mathbf{4} \text { in ppm } \\
\text { (multiplicity, } J \text { in } \\
\text { Hz) }\end{array}$ & $\begin{array}{l}{ }^{13} \mathrm{C} \text { NMR data of } \\
\text { Dechloro- } \\
\text { greensporone F in } \\
\text { ppm }\end{array}$ & $\begin{array}{c}{ }^{13} \mathrm{C} \text { NMR data } \\
\text { Synthetic Product } \\
\mathbf{4} \\
\text { in ppm }\end{array}$ \\
\hline 1 & $1.32(\mathrm{~d}, 6.3)$ & $1.28(\mathrm{~d}, 6.5)$ & 20.9 & 18.6 \\
\hline 2 & $5.26(\mathrm{~m})$ & $5.38(\mathrm{~m})$ & 72.7 & 70.7 \\
\hline 3 & $1.83(\mathrm{~m})$ & & 33.0 & 31.6 \\
\hline 4 & $\begin{array}{l}1.51(\mathrm{~m}) \\
1.96(\mathrm{~m})\end{array}$ & $\begin{array}{l}1.97(\mathrm{~m}) \\
2.02(\mathrm{~m})\end{array}$ & 31.3 & 31.7 \\
\hline 5 & $3.81(\mathrm{~m})$ & $3.93(\mathrm{~m})$ & 79.5 & 79.4 \\
\hline 6 & $1.50(\mathrm{~m})$ & $1.53(\mathrm{~m})$ & 33.5 & 30.5 \\
\hline 7 & $\begin{array}{l}1.65(\mathrm{~m}) \\
1.94(\mathrm{~m})\end{array}$ & $\begin{array}{l}1.97(\mathrm{~m}) \\
1.62(\mathrm{~m}) \\
\end{array}$ & 30.5 & 30.3 \\
\hline 8 & $4.14(\mathrm{~m})$ & $\begin{array}{l}4.33(\mathrm{qd}, 7.2 ., \\
4.2)\end{array}$ & 76.1 & 77.1 \\
\hline 9 & $\begin{array}{l}2.55(\mathrm{dd}, 13.2,8.0) \\
2.62(\mathrm{dd}, 13.2,3.4)\end{array}$ & $2.53(\mathrm{qd}, 12.8,6.0)$ & 47.9 & 47.7 \\
\hline 10 & & & 207.7 & 208.5 \\
\hline 11 & $\begin{array}{l}3.90(\mathrm{~d}, 17.2) \\
3.99(\mathrm{~d}, 17.2)\end{array}$ & $\begin{array}{c}3.75(\mathrm{~d}, 17.1) \\
4.02(\mathrm{~d}, 17.1)\end{array}$ & 49.0 & 49.2 \\
\hline 12 & & & 134.2 & 133.5 \\
\hline 13 & $6.25(\mathrm{~d}, 2.3)$ & $6.12(\mathrm{~d}, 2.0)$ & 109.2 & 109.0 \\
\hline 14 & $5.62(\mathrm{br} \mathrm{s})(\mathrm{OH})$ & $6.53(\mathrm{br} \mathrm{s})(\mathrm{OH})$ & 157.7 & 157.8 \\
\hline 15 & $6.34(\mathrm{~d}, 2.3)$ & $6.31(\mathrm{~d}, 2.1)$ & 98.3 & 98.3 \\
\hline 16 & & & 159.0 & 158.3 \\
\hline 17 & & & 117.3 & 117.8 \\
\hline 18 & & & 167.7 & 167.7 \\
\hline 19 & $3.77(\mathrm{~s})$ & $3.76(\mathrm{~s})$ & 56.0 & 55.8 \\
\hline
\end{tabular}


Table S4. Comparison of ${ }^{1} \mathrm{H}$ and ${ }^{13} \mathrm{C}$ NMR data of Greensporone F with the Synthetic 5

\begin{tabular}{|c|c|c|c|c|}
\hline Position & $\begin{array}{l}{ }^{1} \mathrm{HNMR} \\
\text { of Greensporone F in } \\
\text { ppm (multiplicity, } J \\
\text { in Hz) }\end{array}$ & $\begin{array}{c}{ }^{1} \text { H NMR } \\
\text { Synthetic Product } \mathbf{5} \text { in } \\
\text { ppm (multiplicity, } J \text { in } \\
\text { Hz) }\end{array}$ & $\begin{array}{c}{ }^{13} \mathrm{C} \text { NMR of } \\
\text { Greensporone } \\
\mathrm{F} \text { in ppm }\end{array}$ & $\begin{array}{c}{ }^{13} \mathrm{C} \text { NMR } \\
\text { of Synthetic } \\
\text { Product } \mathbf{5} \\
\text { in ppm }\end{array}$ \\
\hline 1 & $1.32(\mathrm{~d}, 6.2)$ & $1.27(\mathrm{~d}, 6.5)$ & 21.5 & 18.5 \\
\hline 2 & $5.21(\mathrm{~m})$ & $5.36(\mathrm{~m})$ & 73.4 & 71.0 \\
\hline 3 & $\begin{array}{l}1.72(\mathrm{~m}) \\
1.90(\mathrm{~m})\end{array}$ & $\begin{array}{l}2.04(\mathrm{~m}), \\
1.95(\mathrm{~m})\end{array}$ & 33.8 & 31.7 \\
\hline 4 & $\begin{array}{l}1.38(\mathrm{~m}) \\
1.79(\mathrm{~m})\end{array}$ & $1.48(\mathrm{~m})$ & 34.7 & 31.7 \\
\hline 5 & $3.79(\mathrm{~m})$ & $3.91(\mathrm{~m})$ & 79.3 & 79.1 \\
\hline 6 & $\begin{array}{l}1.55(\mathrm{~m}) \\
2.02(\mathrm{~m})\end{array}$ & $1.66(\mathrm{~m})$ & 31.6 & 30.2 \\
\hline 7 & $\begin{array}{l}1.75(\mathrm{~m}) \\
1.91(\mathrm{~m})\end{array}$ & $\begin{array}{l}1.91(\mathrm{~m}) \\
1.95(\mathrm{~m})\end{array}$ & 29.8 & 29.6 \\
\hline 8 & $4.33(\mathrm{~m})$ & $4.38(\mathrm{~m})$ & 76.3 & 76.9 \\
\hline 9 & $\begin{array}{l}2.53(\mathrm{dd}, 13.5,6.9) \\
2.81(\mathrm{dd}, 13.5,4.6)\end{array}$ & $\begin{array}{l}2.46(\mathrm{dd}, 13.3,6.4) \\
2.84(\mathrm{dd}, 13.2,4.9)\end{array}$ & 46.6 & 46.3 \\
\hline 10 & & & 203.9 & 204.9 \\
\hline 11 & $\begin{array}{l}3.99(\mathrm{~d}, 18.5) \\
4.13(\mathrm{~d}, 18.5) \\
\end{array}$ & $4.04(\mathrm{~d}, 0.7)$ & 48.6 & 47.7 \\
\hline 12 & & & 132.1 & 131.3 \\
\hline 13 & & & 113.1 & 112.6 \\
\hline 14 & $5.70(\mathrm{~s})(\mathrm{OH})$ & $5.83(\mathrm{br} \mathrm{s})(\mathrm{OH})$ & 153.3 & 152.9 \\
\hline 15 & $6.59(\mathrm{~s})$ & $6.57(\mathrm{~s})$ & 99.1 & 98.8 \\
\hline 16 & & & 157.0 & 156.1 \\
\hline 17 & & & 119.2 & 119.4 \\
\hline 18 & & & 166.9 & 166.7 \\
\hline 19 & $3.77(\mathrm{~s})$ & $3.78(\mathrm{~s})$ & 56.4 & 56.0 \\
\hline
\end{tabular}




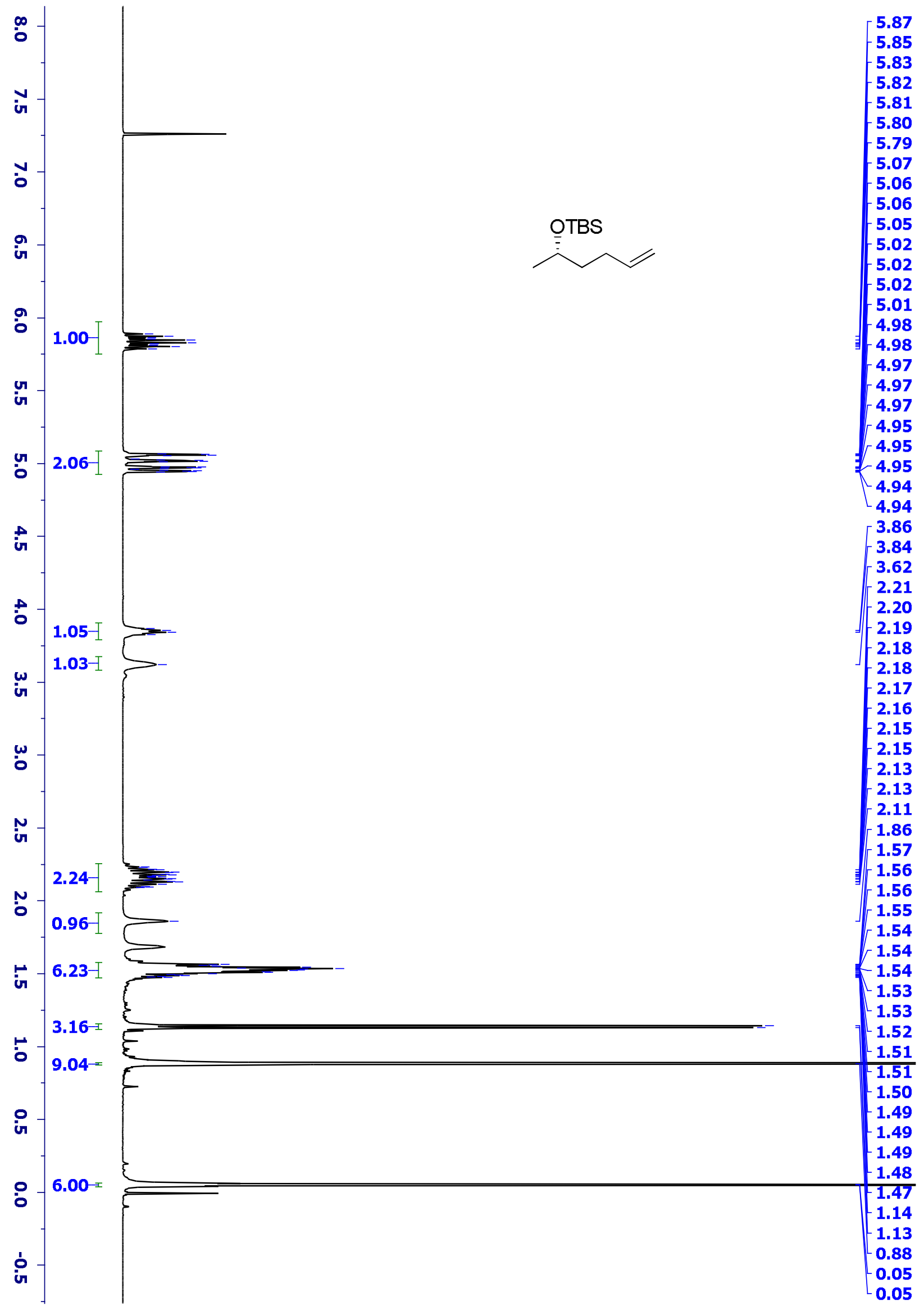

${ }^{1} \mathrm{H}$ NMR spectrum of 15 (400 MHz, $\mathrm{CDCl}_{3}$ ) 


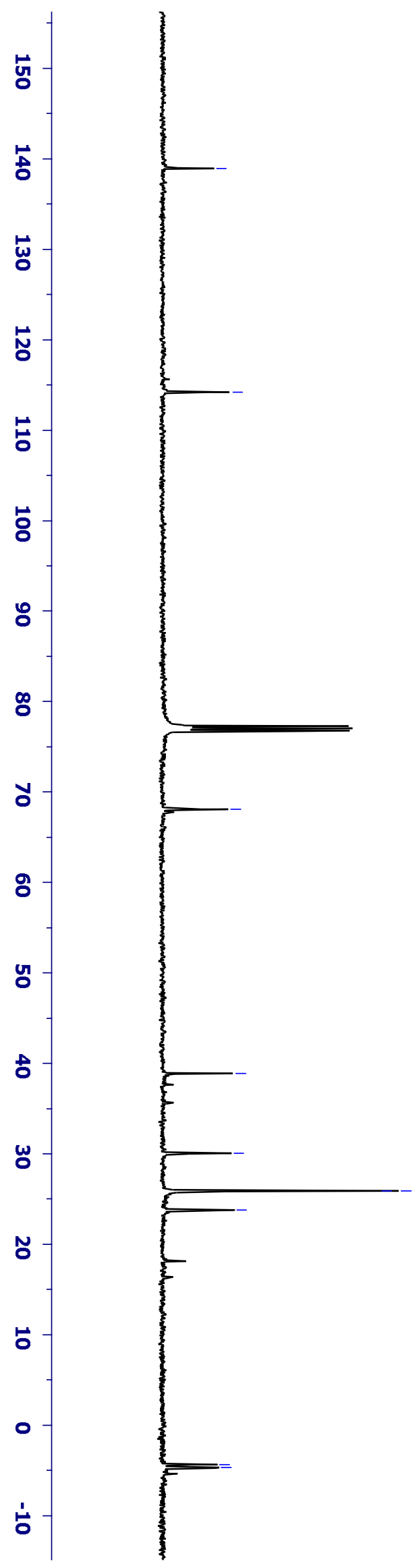

$-138.94$

$-114.21$

OTBS

$-38.89$

$-30.07$

25.92

25.91

23.78

${ }^{13} \mathrm{C}$ NMR spectrum of $\mathbf{1 5}\left(100 \mathrm{MHz}, \mathrm{CDCl}_{3}\right)$ 


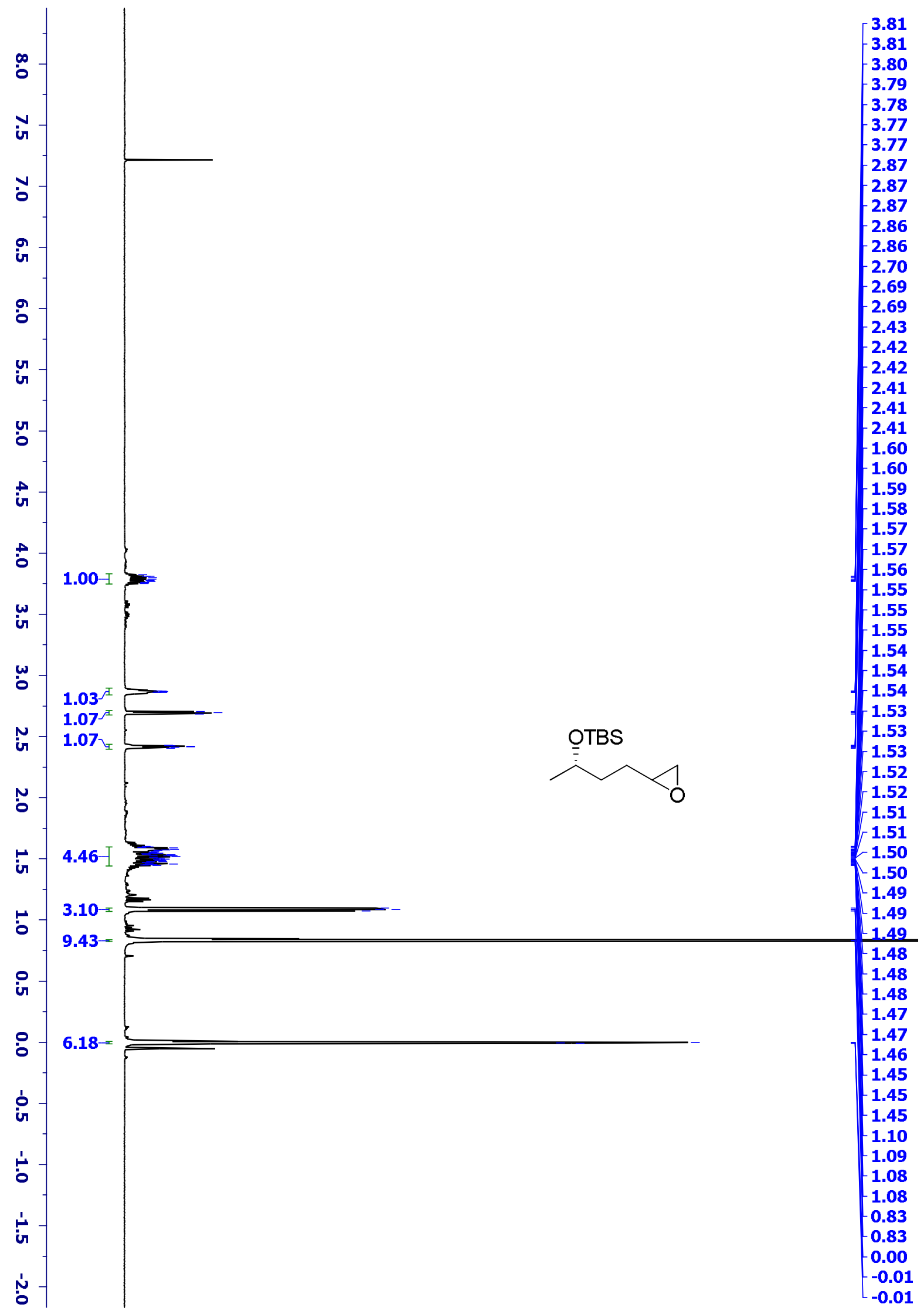

${ }^{1} \mathrm{H}$ NMR spectrum of $\mathbf{1 6}\left(500 \mathrm{MHz}, \mathrm{CDCl}_{3}\right)$ 


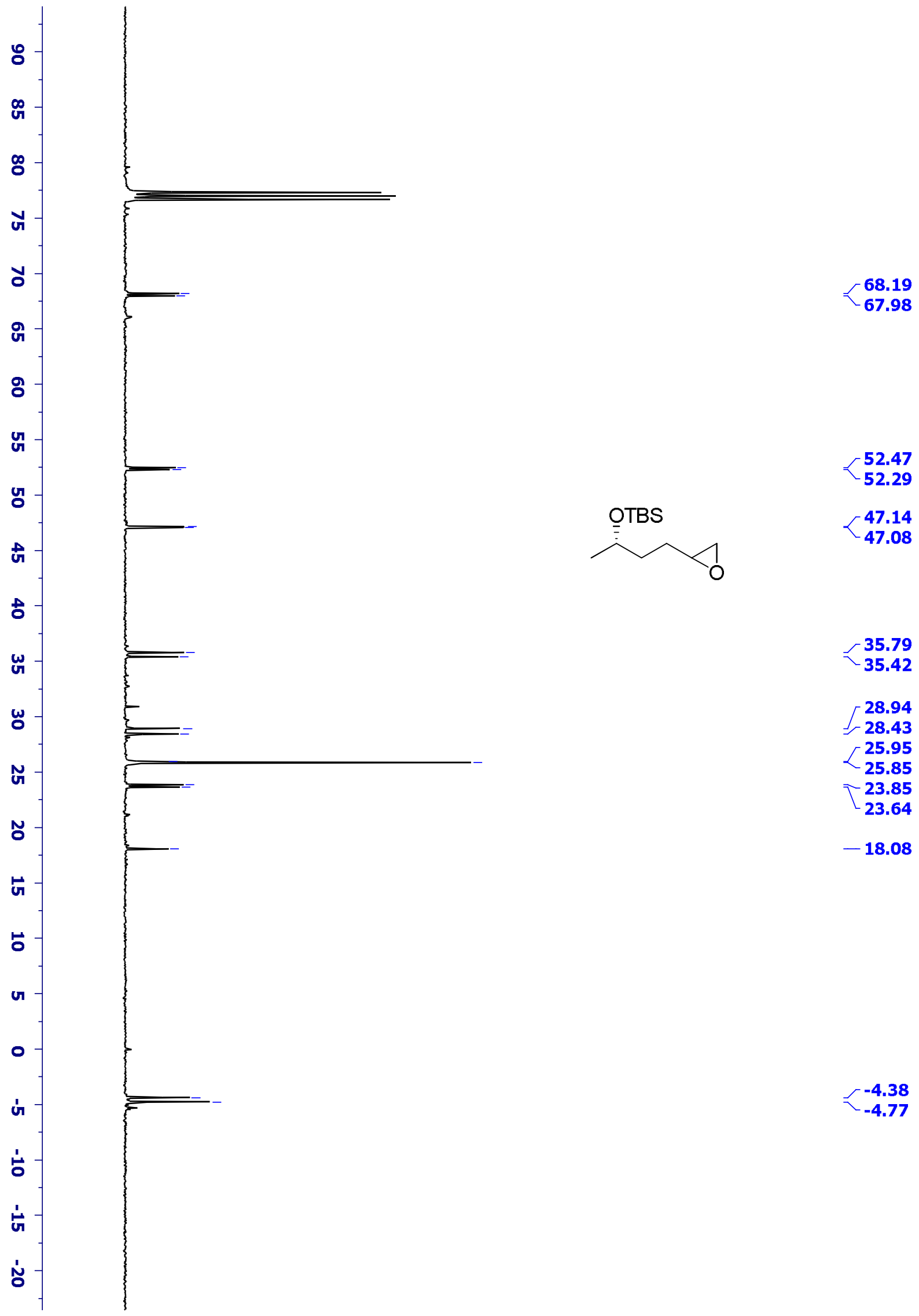

${ }^{13} \mathrm{C}$ NMR spectrum of $\mathbf{1 6}\left(100 \mathrm{MHz}, \mathrm{CDCl}_{3}\right)$ 


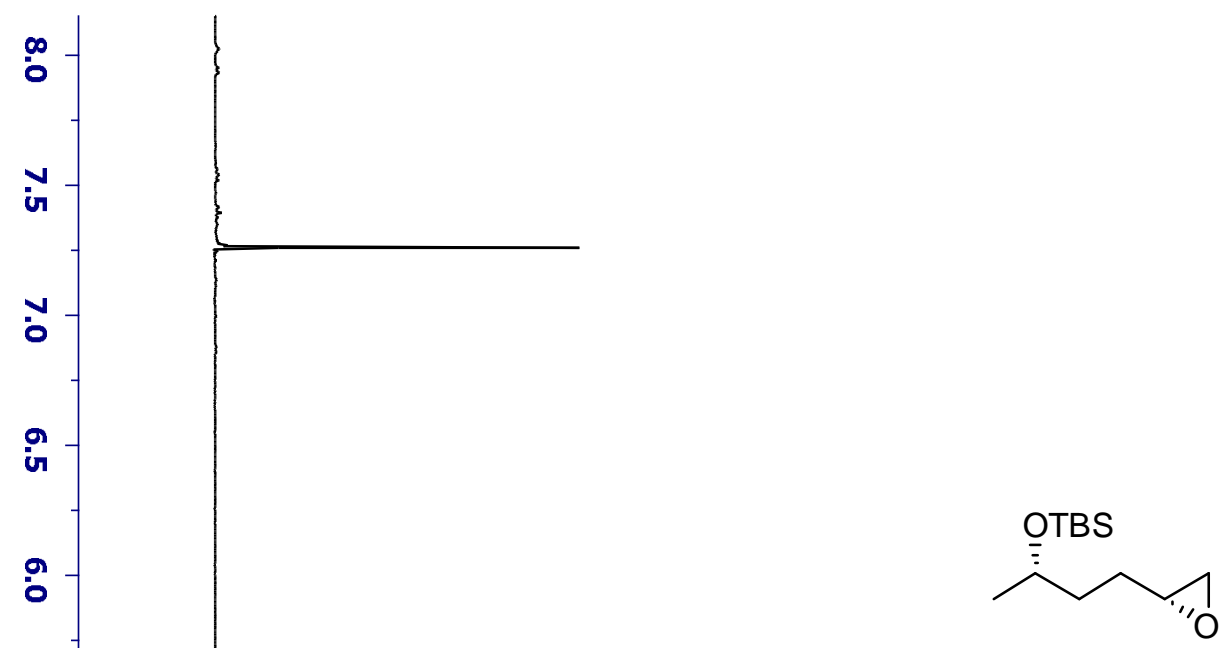

on

in

0

ir

$1.00-$

i

$\omega$

$0.86-\simeq$

in

0.89

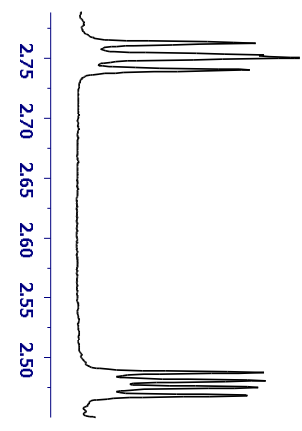

3.89

3.87

3.86

3.86

3.85

3.83

3.82

2.92

2.92

2.76

2.75

2.74

2.73

2.48

2.47

2.47

궁

它

4.13-

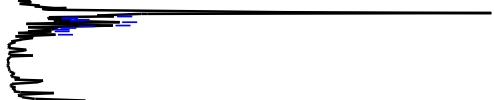

1.58

1.58

1.56

1.54

1:54

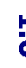

3.05

9.03

$E$

1.12

0.88

is

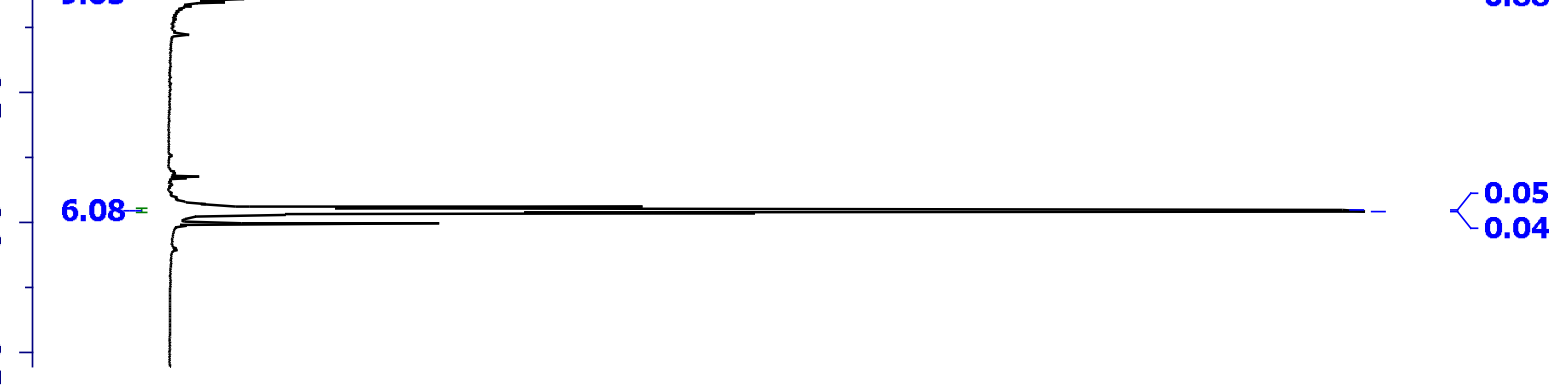

${ }^{1} \mathrm{H}$ NMR spectrum of $17\left(400 \mathrm{MHz}, \mathrm{CDCl}_{3}\right)$ 


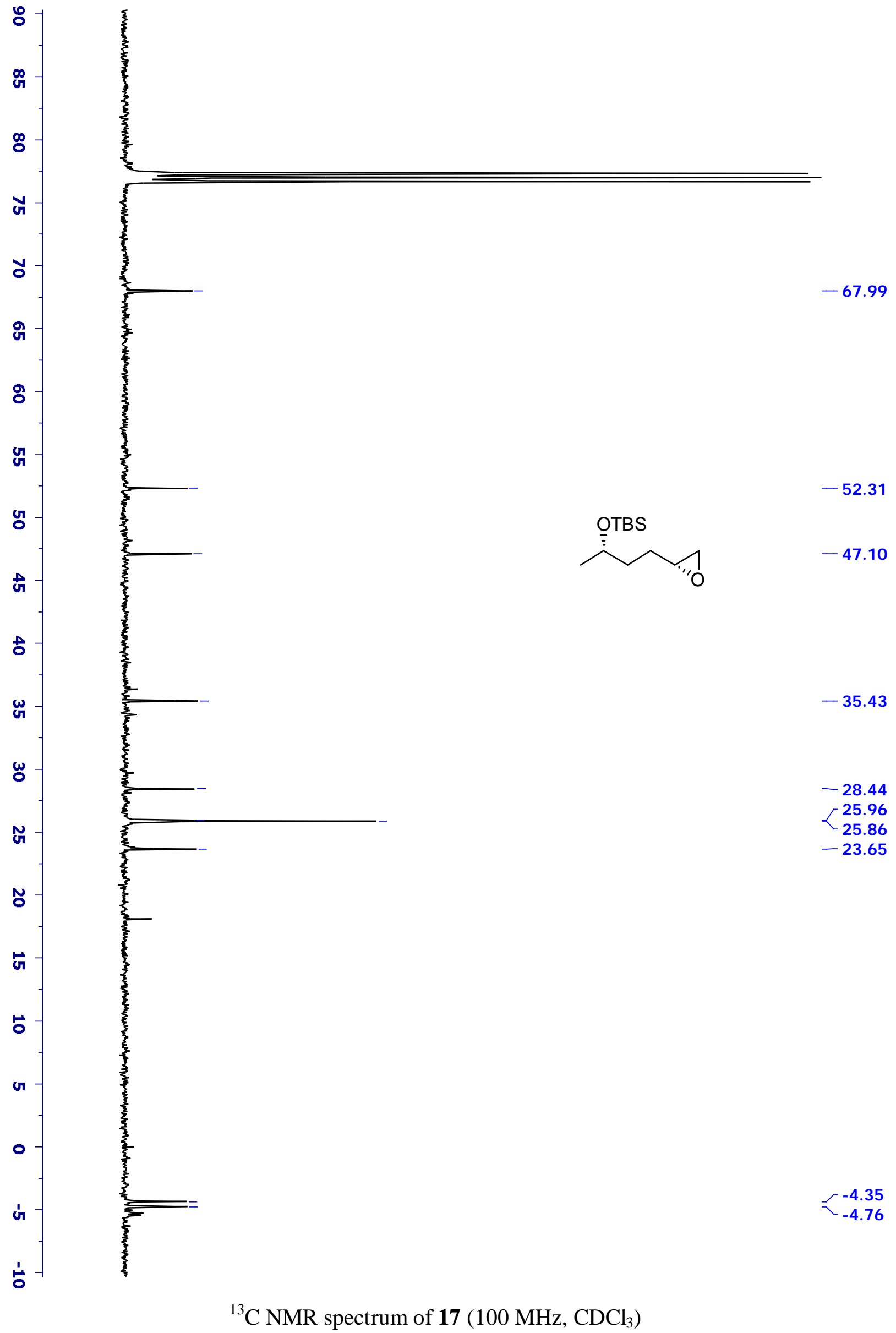




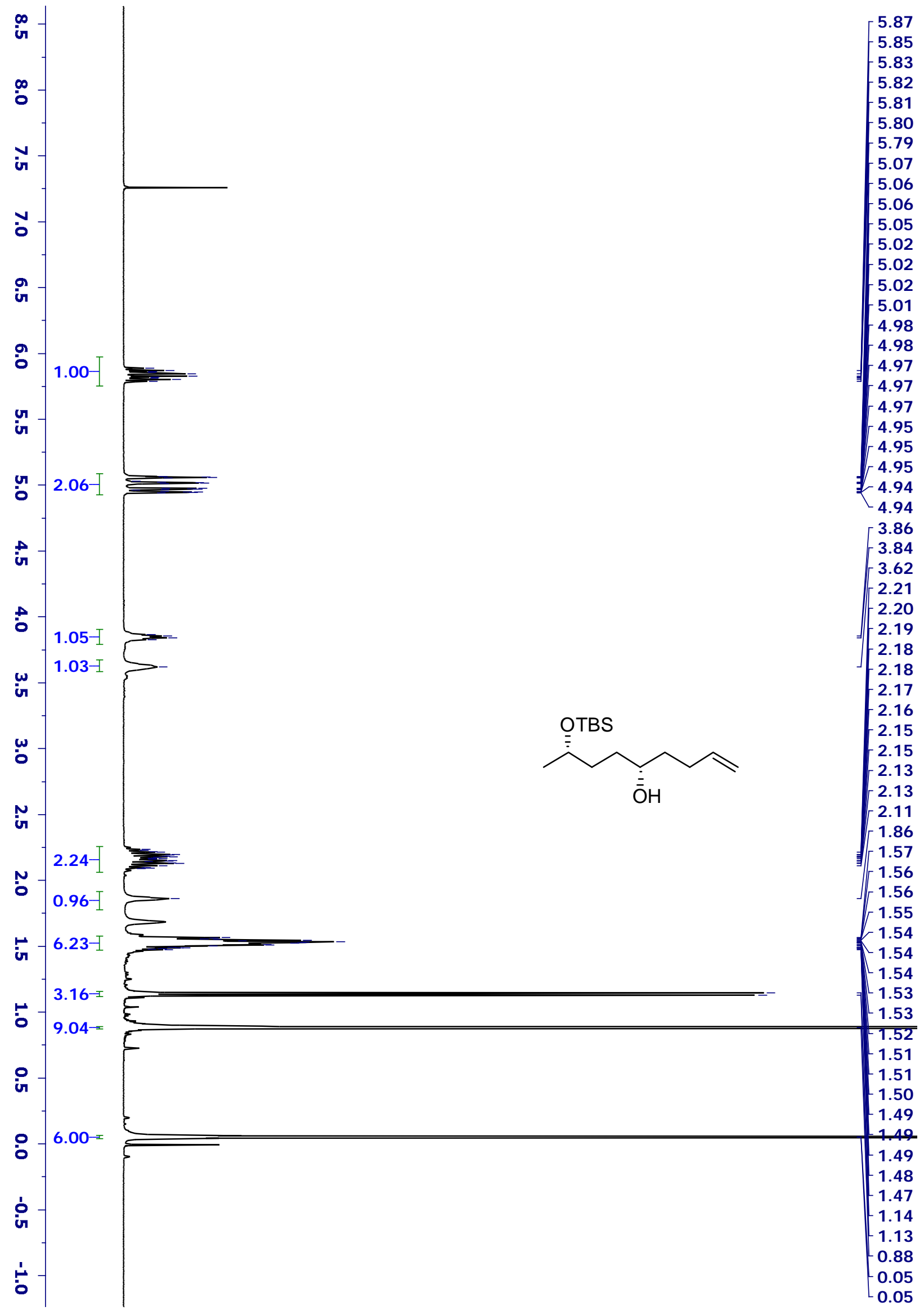

${ }^{1} \mathrm{H}$ NMR spectrum of $\mathbf{1 8}\left(400 \mathrm{MHz}, \mathrm{CDCl}_{3}\right)$ 


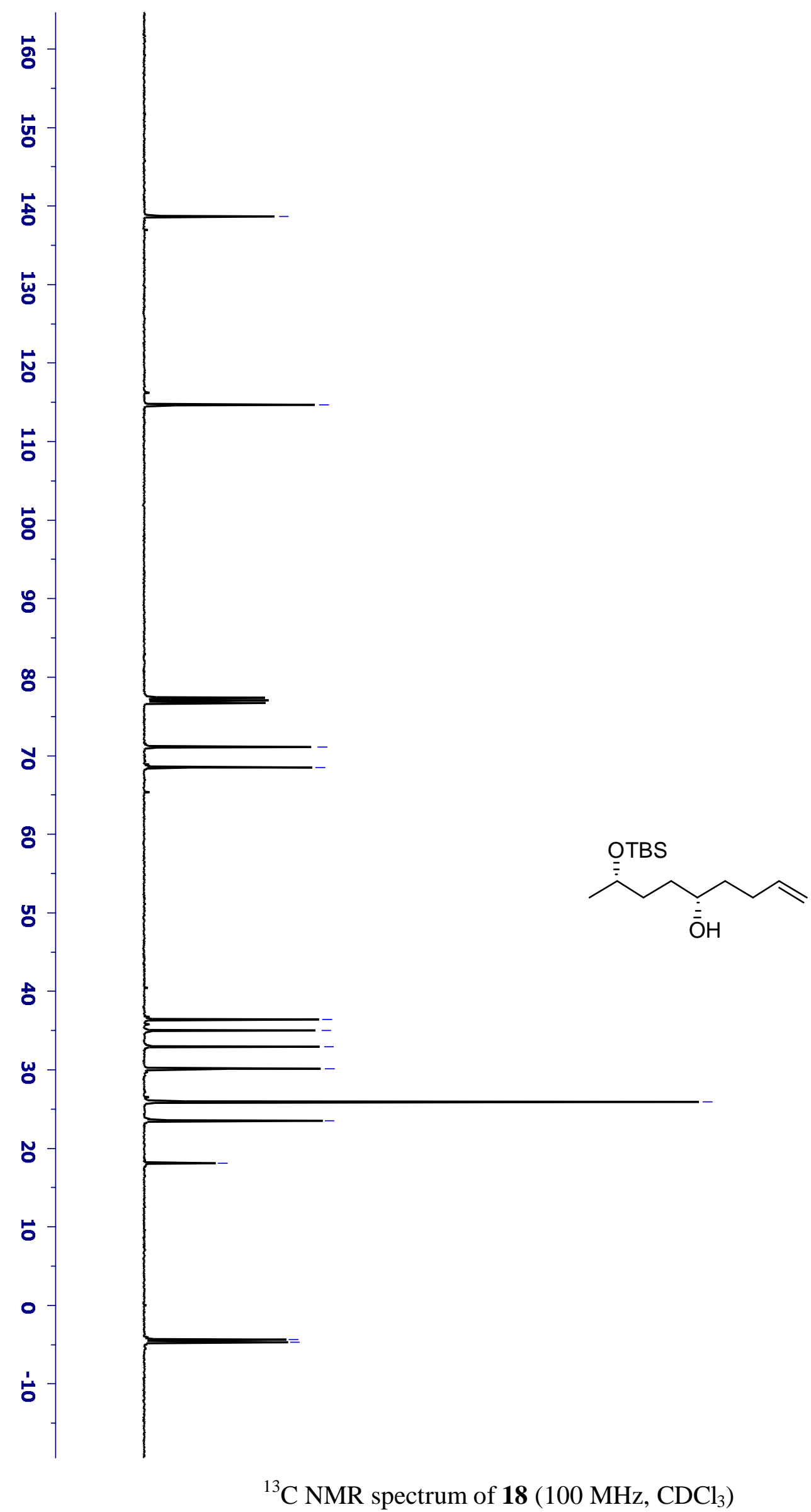

$-138.68$

$-114.65$

$-71.12$

$-68.51$

$-36.39$

34.99

$-32.95$

30.12

25.91

23.51

$-18.12$

$-4.39$

$-4.68$

${ }^{13} \mathrm{C}$ NMR spectrum of $\mathbf{1 8}\left(100 \mathrm{MHz}, \mathrm{CDCl}_{3}\right)$ 


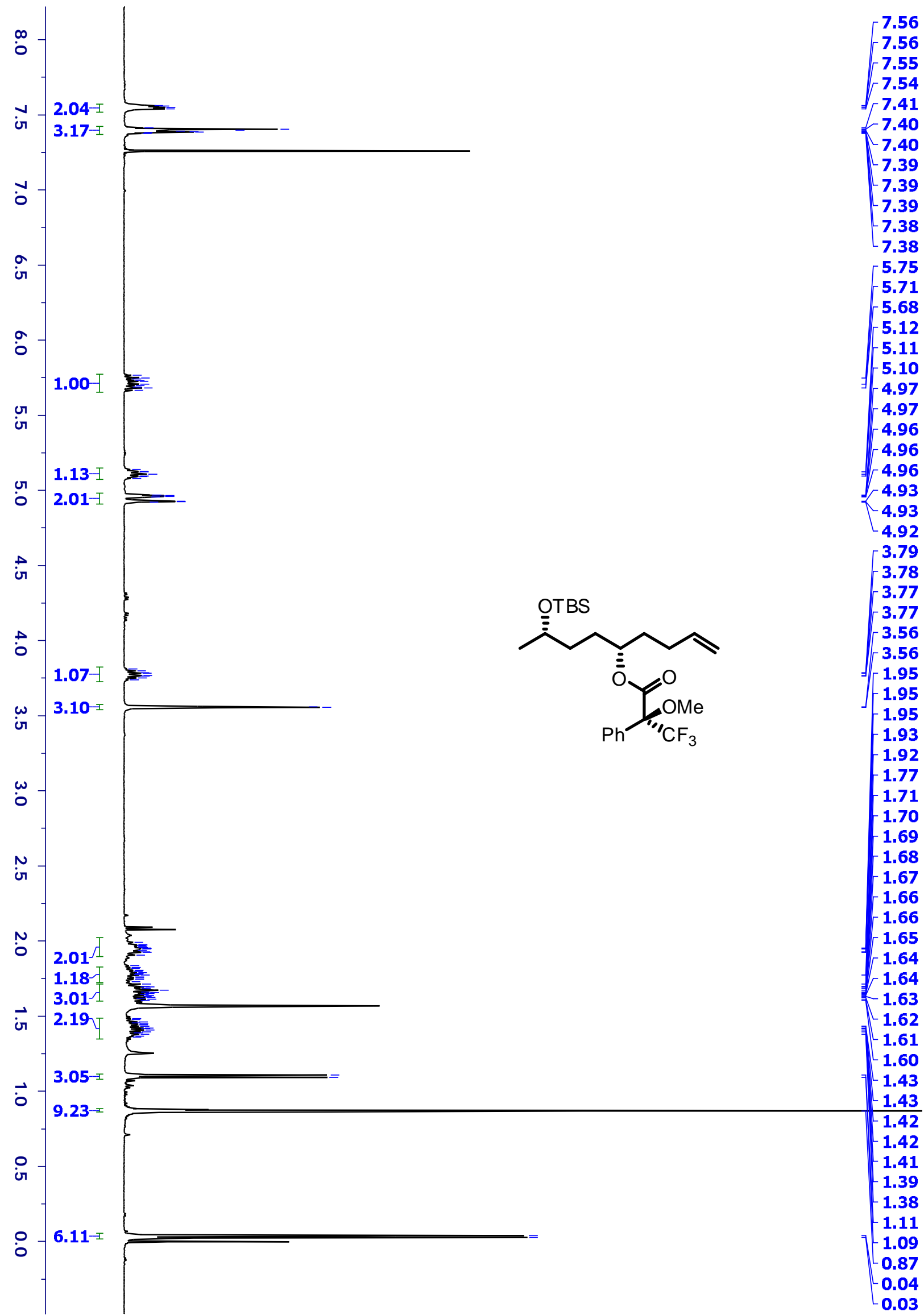

${ }^{1} \mathrm{H}$ NMR spectrum of $\mathbf{1 8}^{\prime}\left(400 \mathrm{MHz}, \mathrm{CDCl}_{3}\right.$ ) 


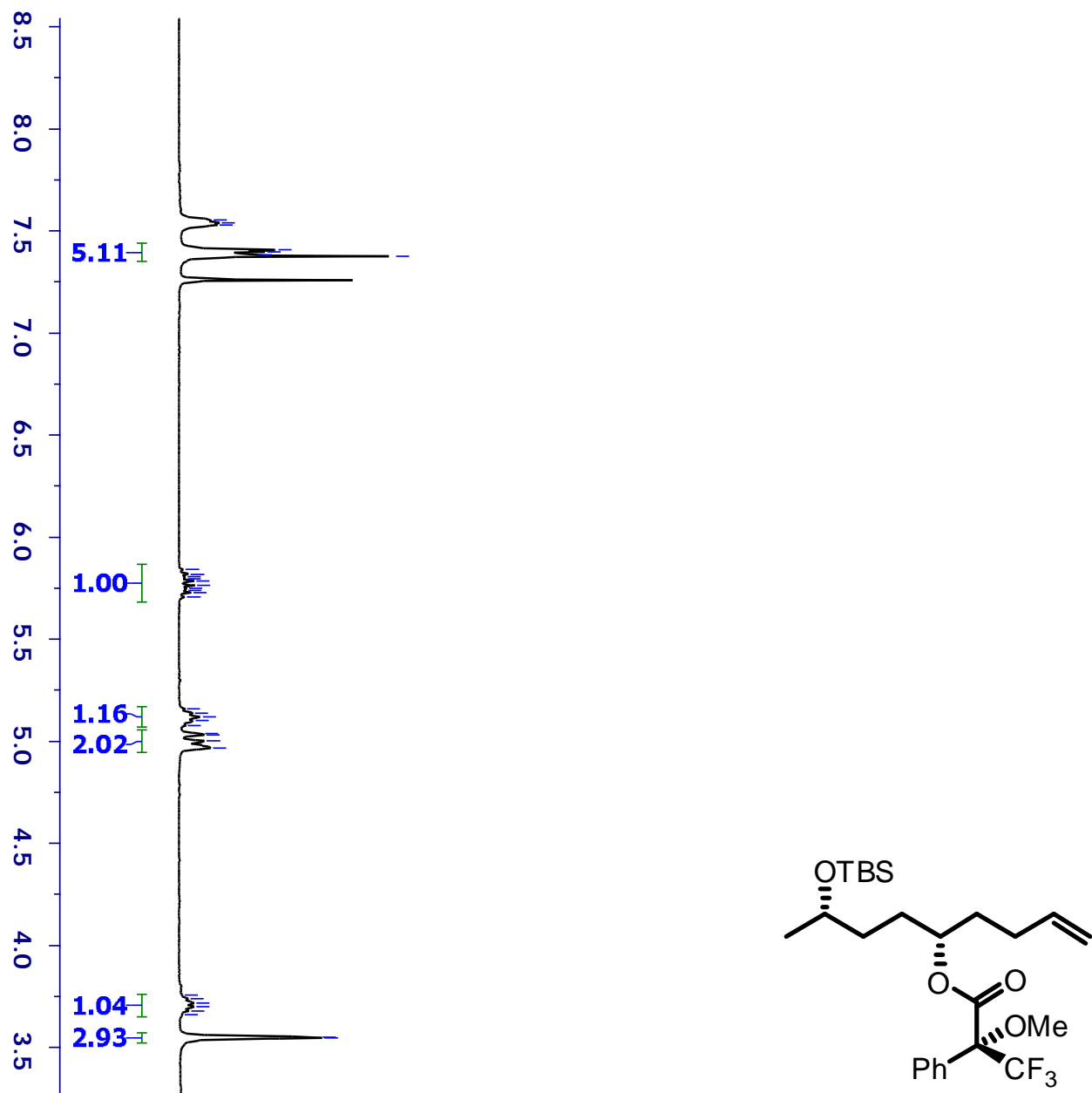

$\ddot{\omega}$

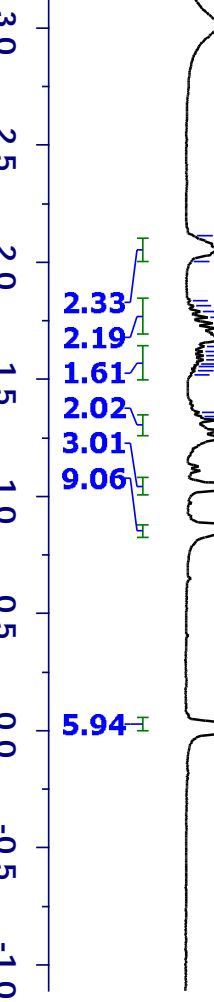

${ }^{1} \mathrm{H}$ NMR spectrum of 18" (300 MHz, $\mathrm{CDCl}_{3}$ ) 


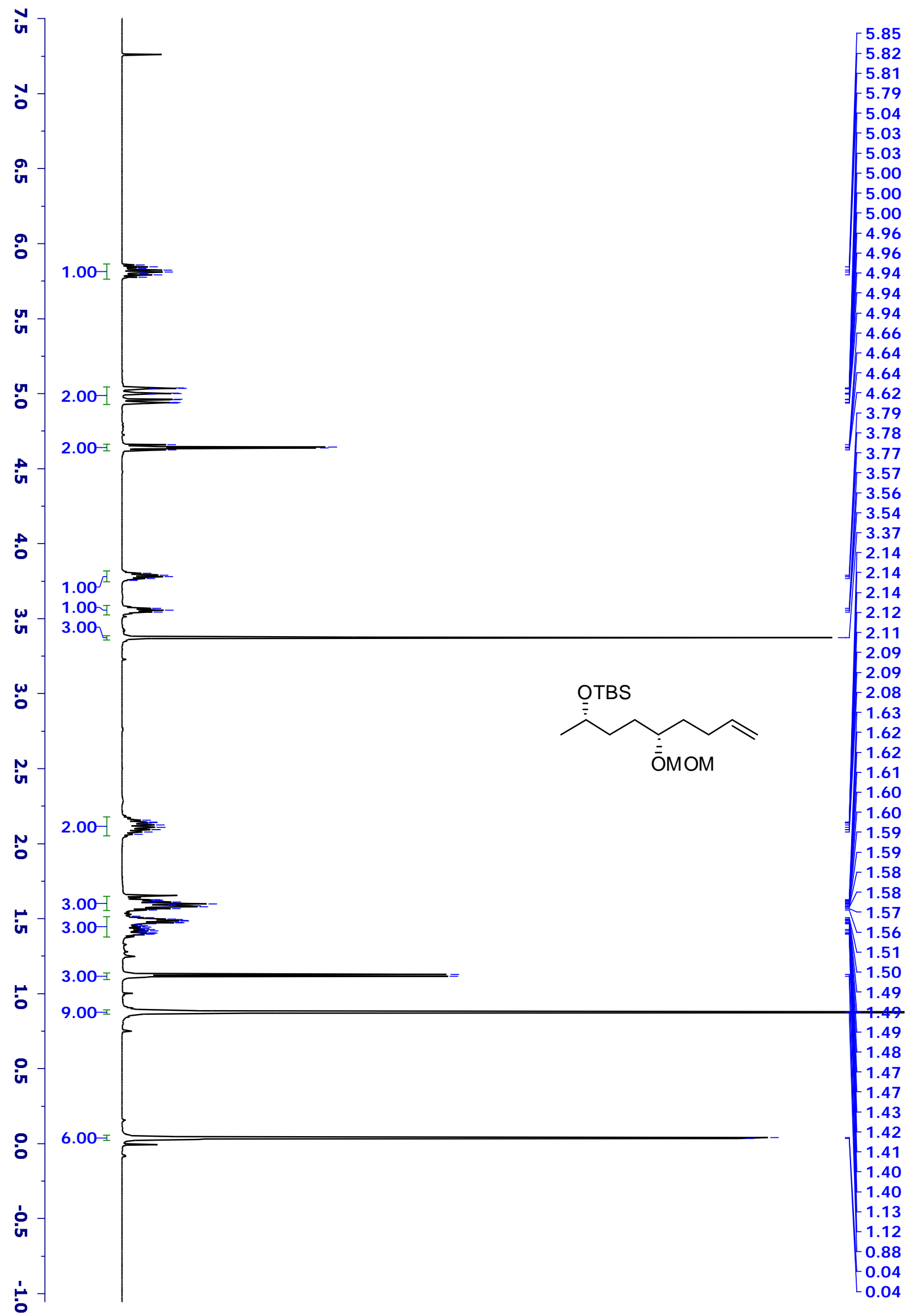

${ }^{1} \mathrm{H}$ NMR spectrum of $\mathbf{1 9}\left(300 \mathrm{MHz}, \mathrm{CDCl}_{3}\right)$ 


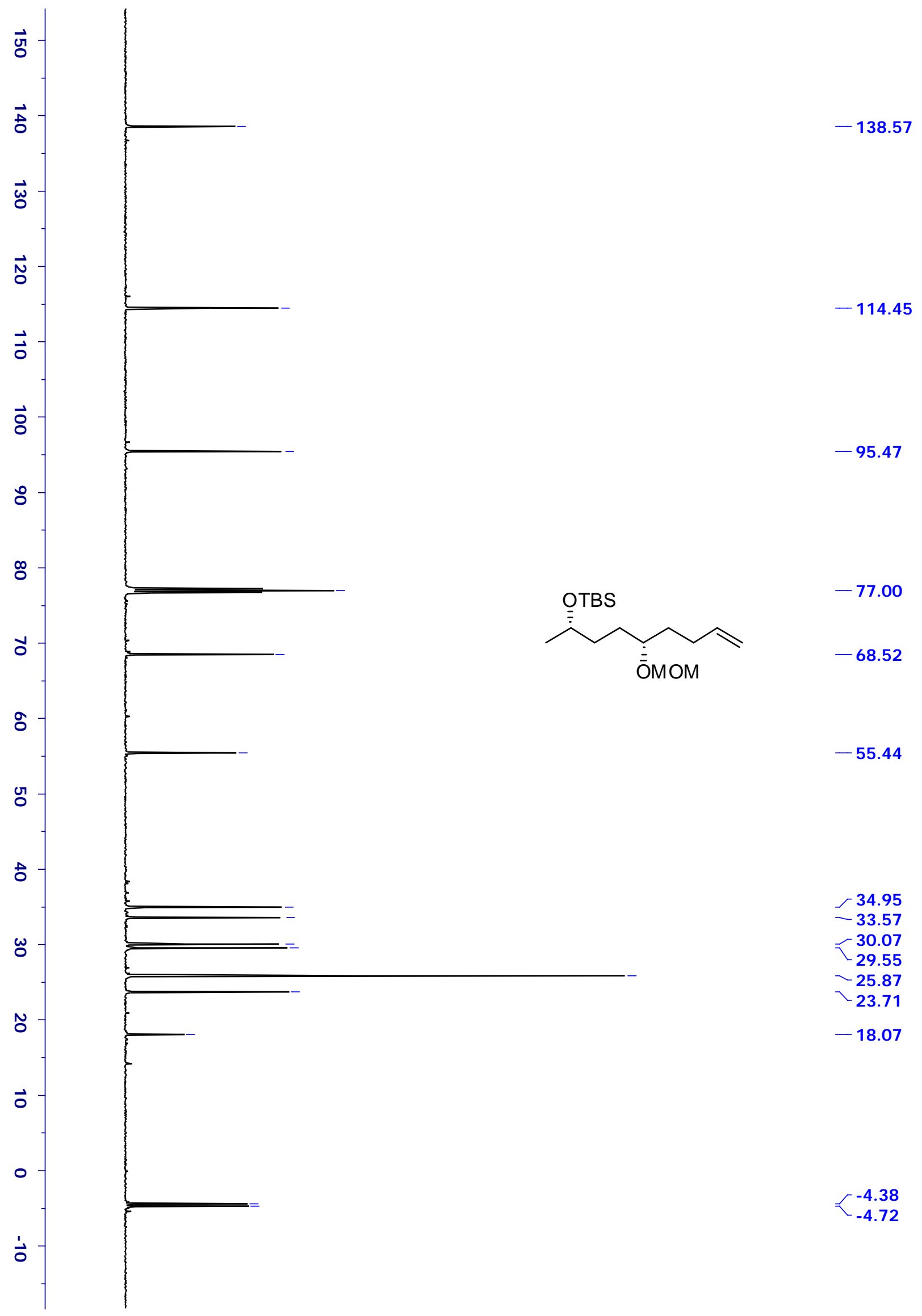

${ }^{13} \mathrm{C}$ NMR spectrum of $\mathbf{1 9}\left(125 \mathrm{MHz}, \mathrm{CDCl}_{3}\right)$ 


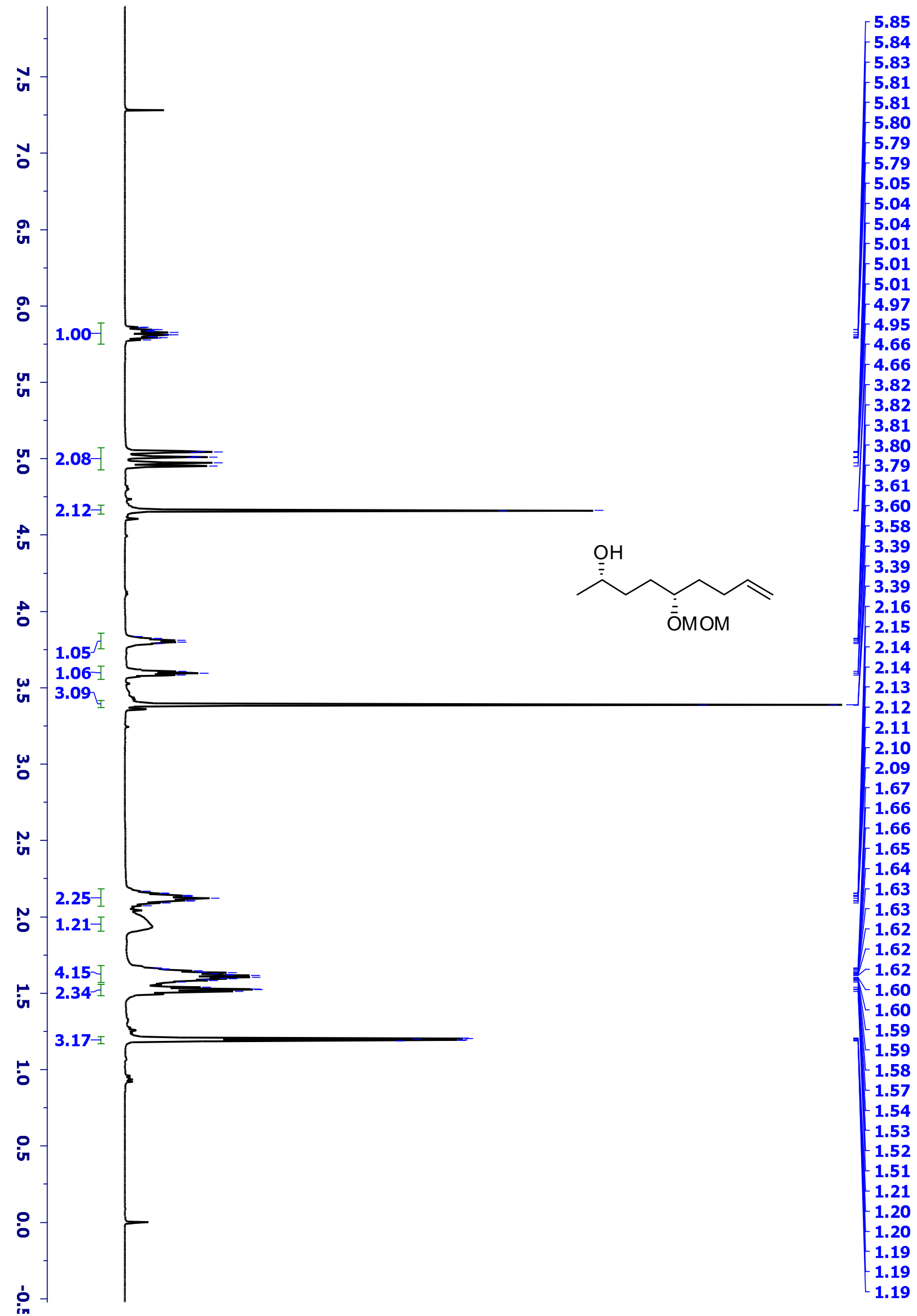

${ }^{1} \mathrm{H}$ NMR spectrum of $\mathbf{1 1}\left(500 \mathrm{MHz}, \mathrm{CDCl}_{3}\right)$ 
Pag e | S24

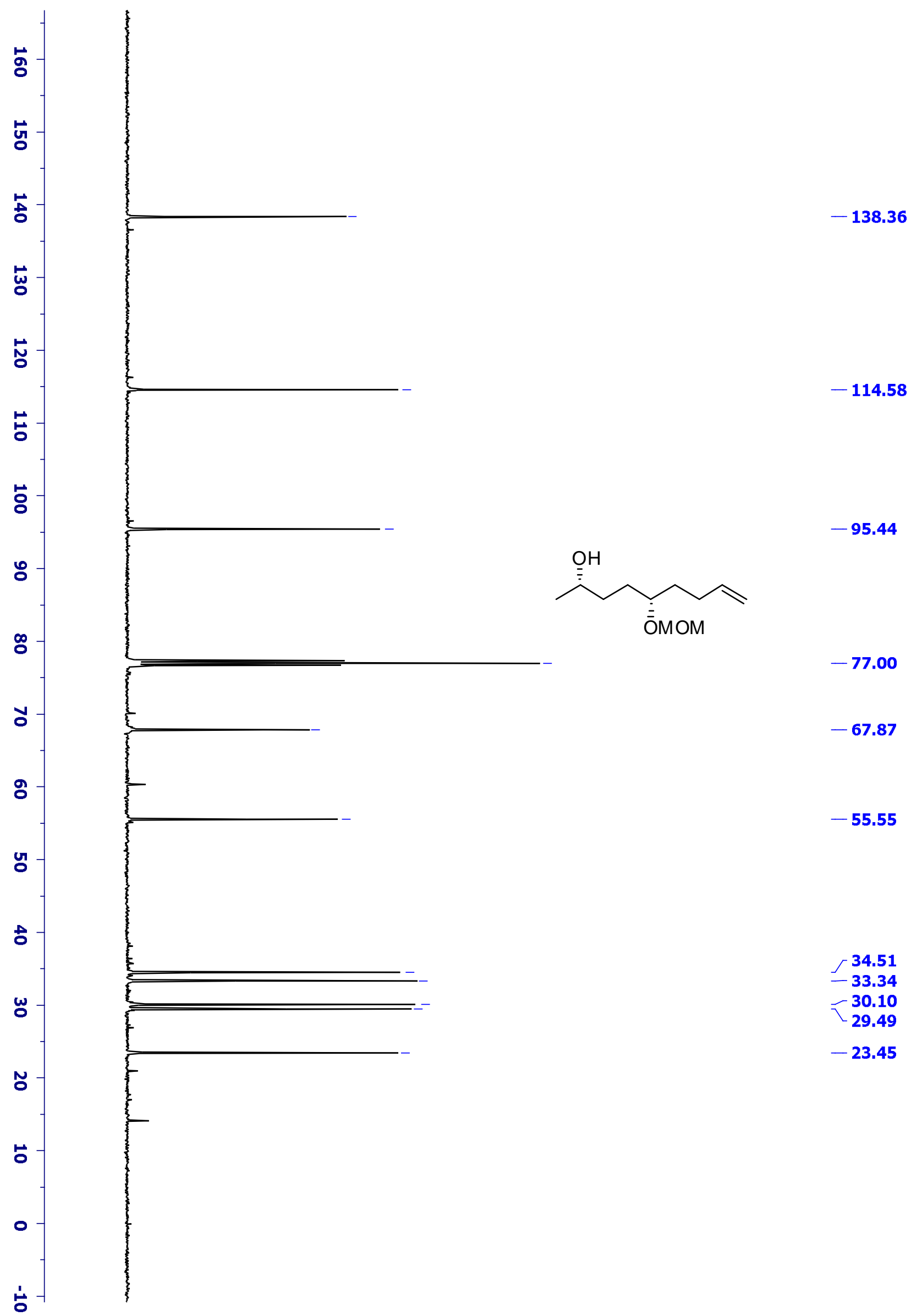

${ }^{13} \mathrm{C}$ NMR spectrum of $\mathbf{1 1}\left(100 \mathrm{MHz}, \mathrm{CDCl}_{3}\right)$ 


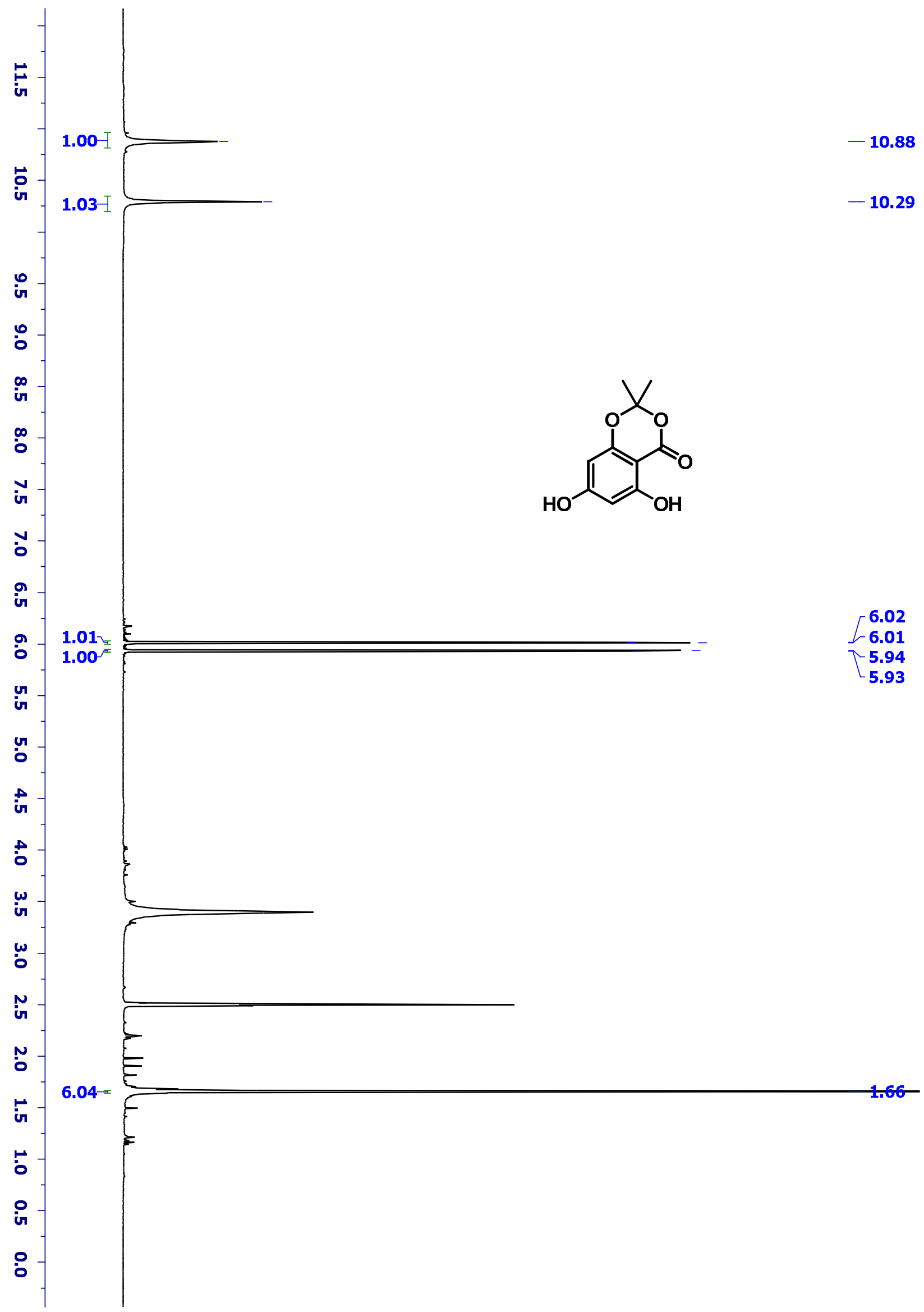

${ }^{1} \mathrm{H}$ NMR spectrum of 20a (400 MHz, DMSO- $d_{6}$ ) 


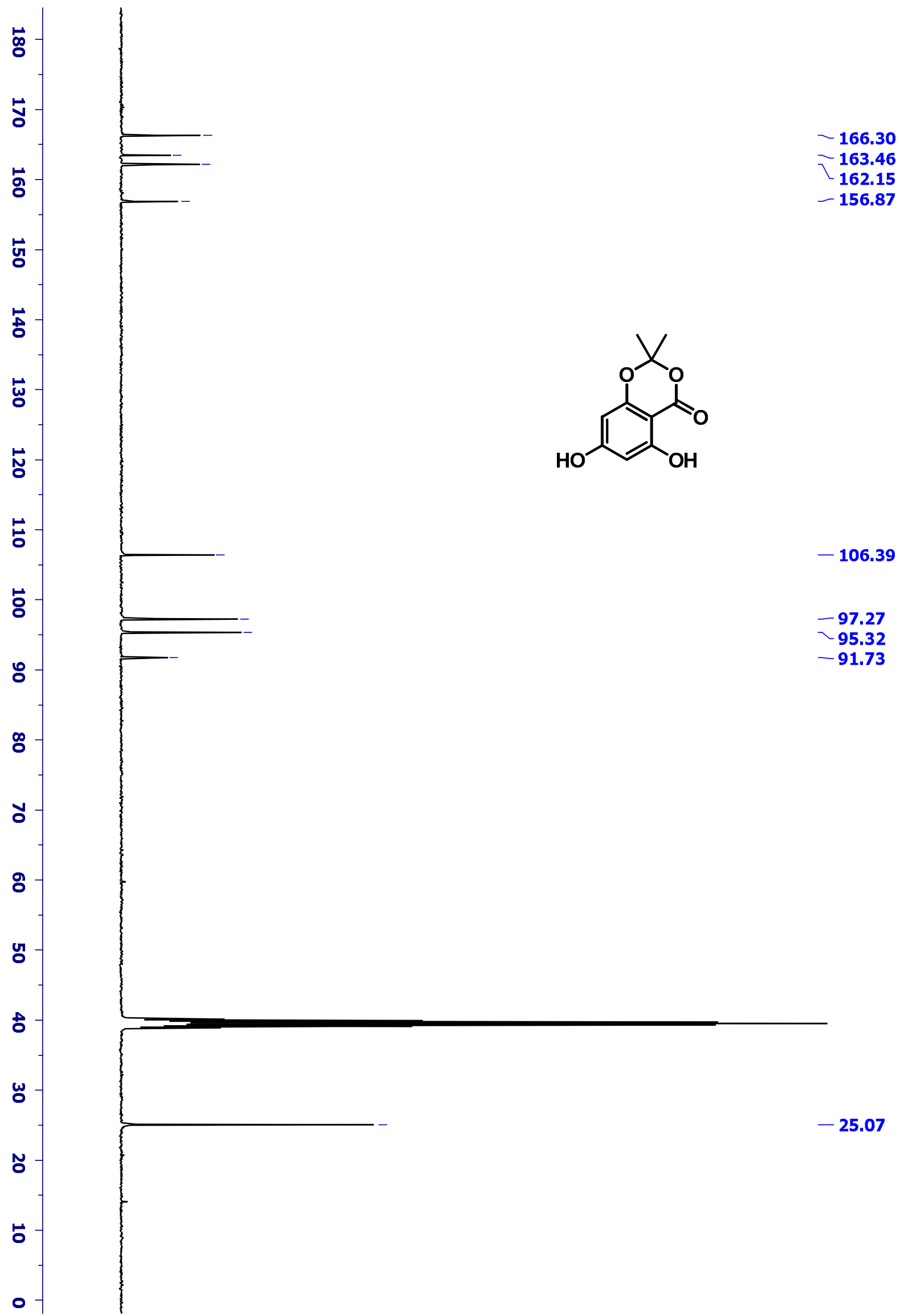

${ }^{13} \mathrm{C}$ NMR spectrum of 20a (100 MHz, DMSO- $\left.d_{6}\right)$ 


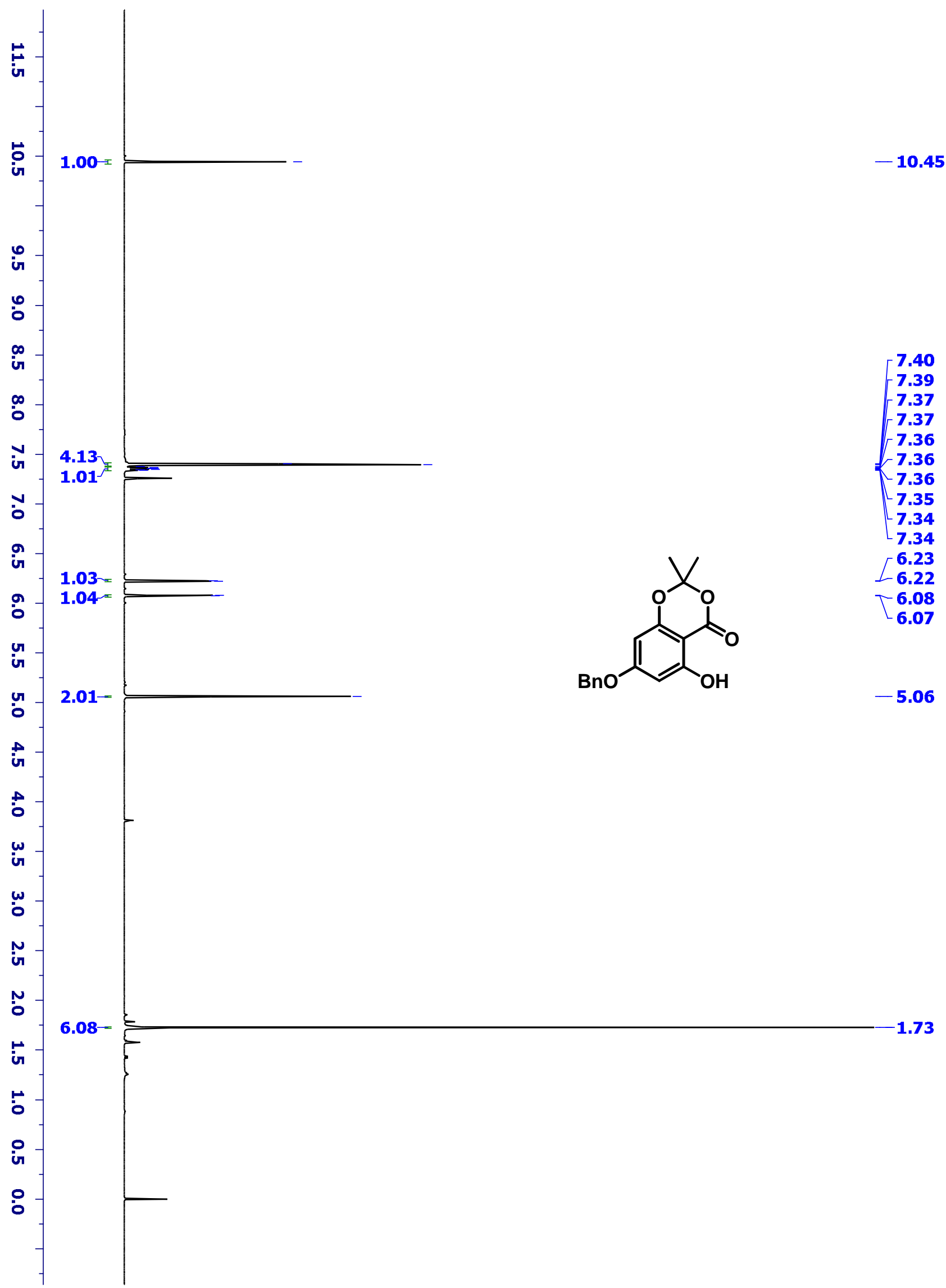

${ }^{1} \mathrm{H}$ NMR spectrum of $\mathbf{2 0 b}\left(500 \mathrm{MHz}, \mathrm{CDCl}_{3}\right.$ ) 


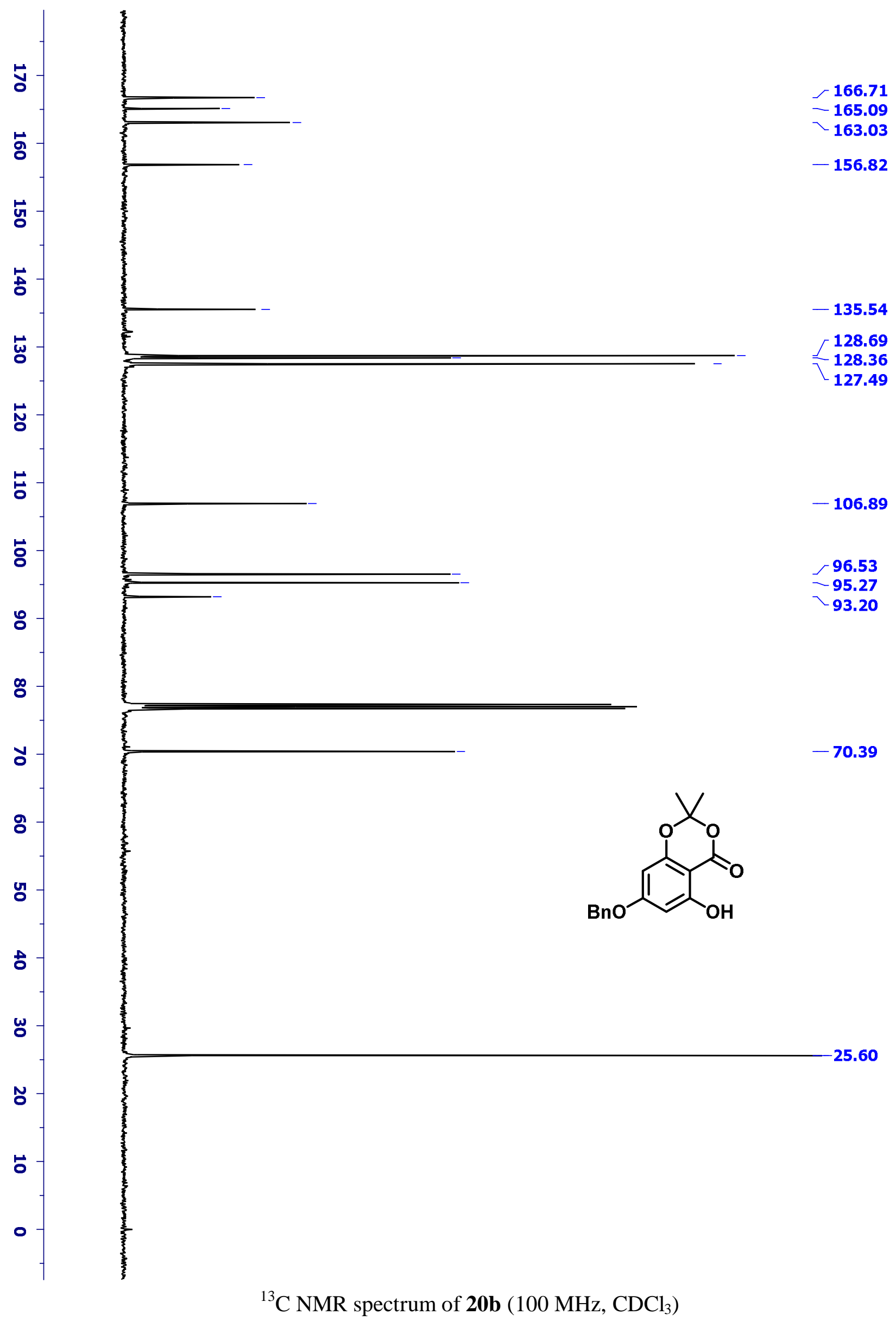




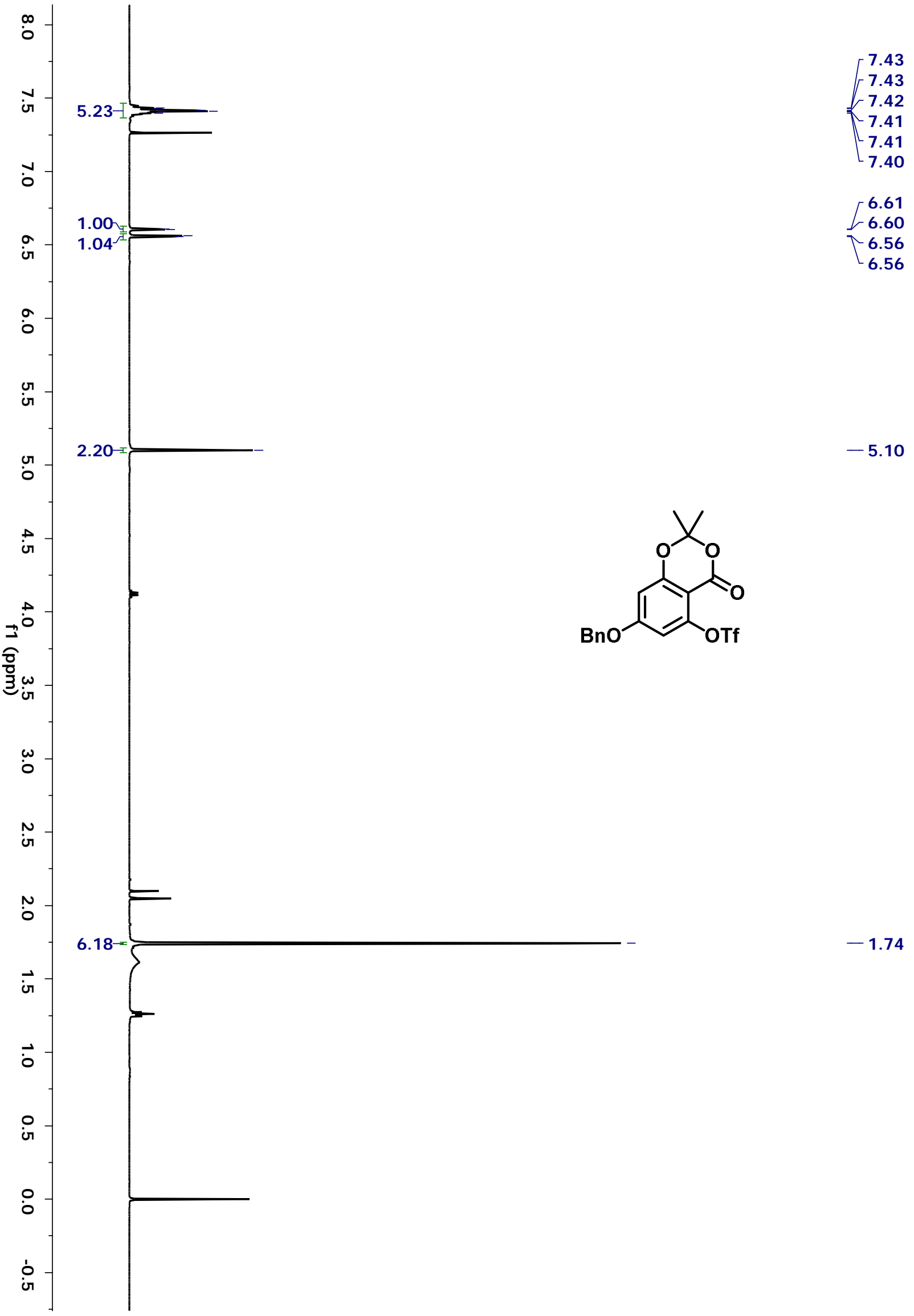

${ }^{1} \mathrm{H}$ NMR spectrum of 20c $\left(500 \mathrm{MHz}, \mathrm{CDCl}_{3}\right)$ 


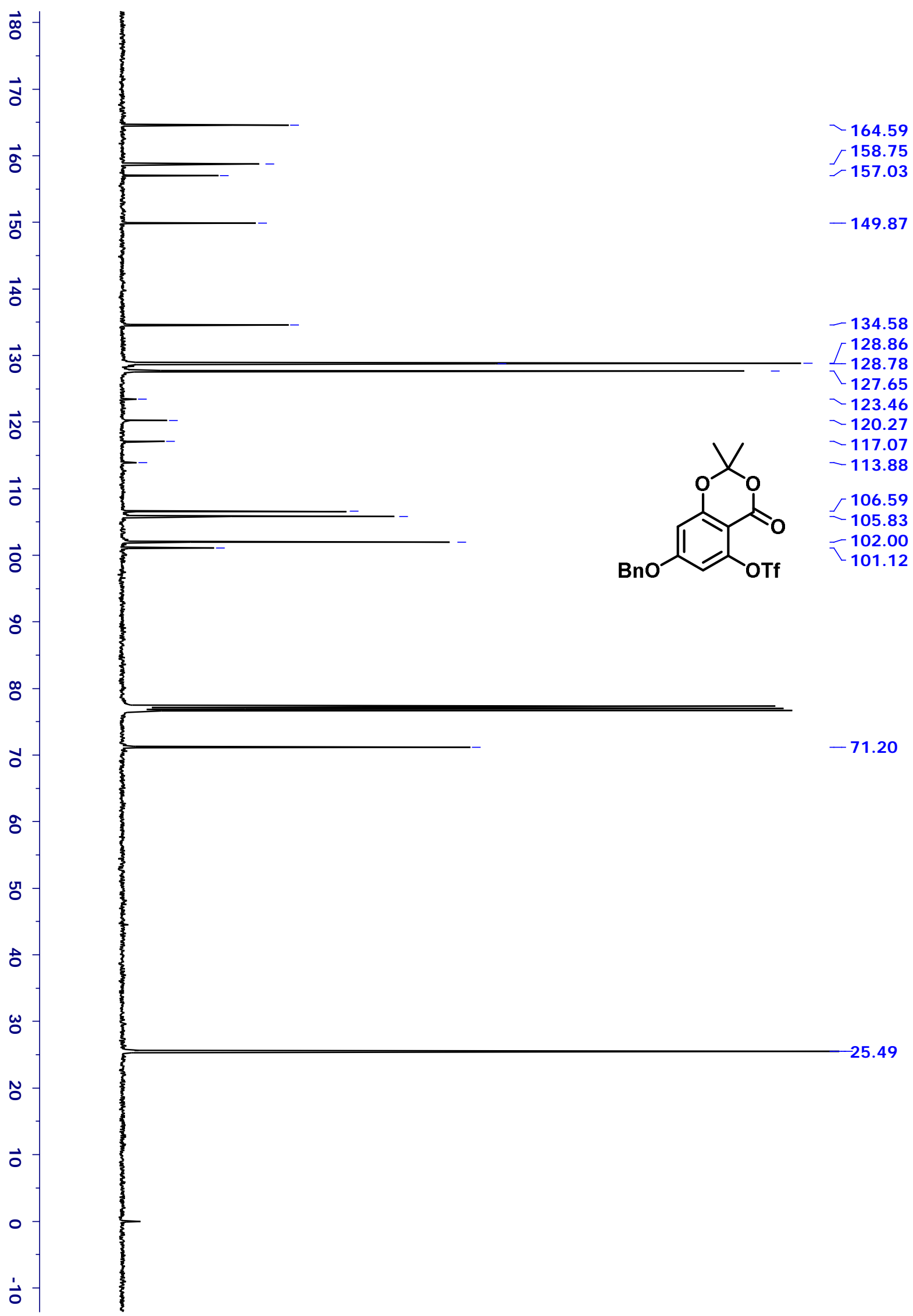

${ }^{13} \mathrm{C}$ NMR spectrum of $20 \mathrm{c}\left(100 \mathrm{MHz}, \mathrm{CDCl}_{3}\right)$ 


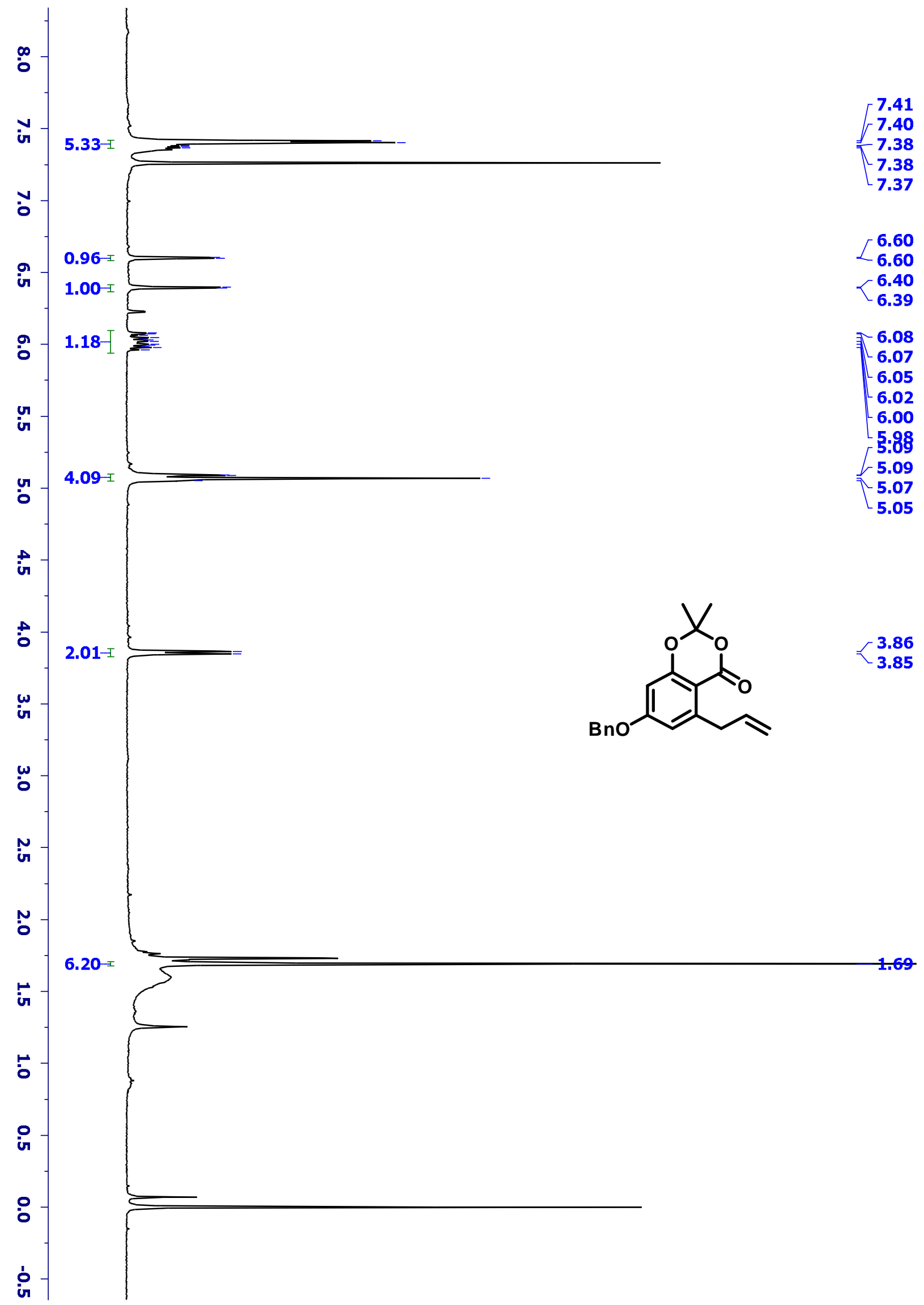

${ }^{1} \mathrm{H}$ NMR spectrum of $12\left(400 \mathrm{MHz}, \mathrm{CDCl}_{3}\right)$ 


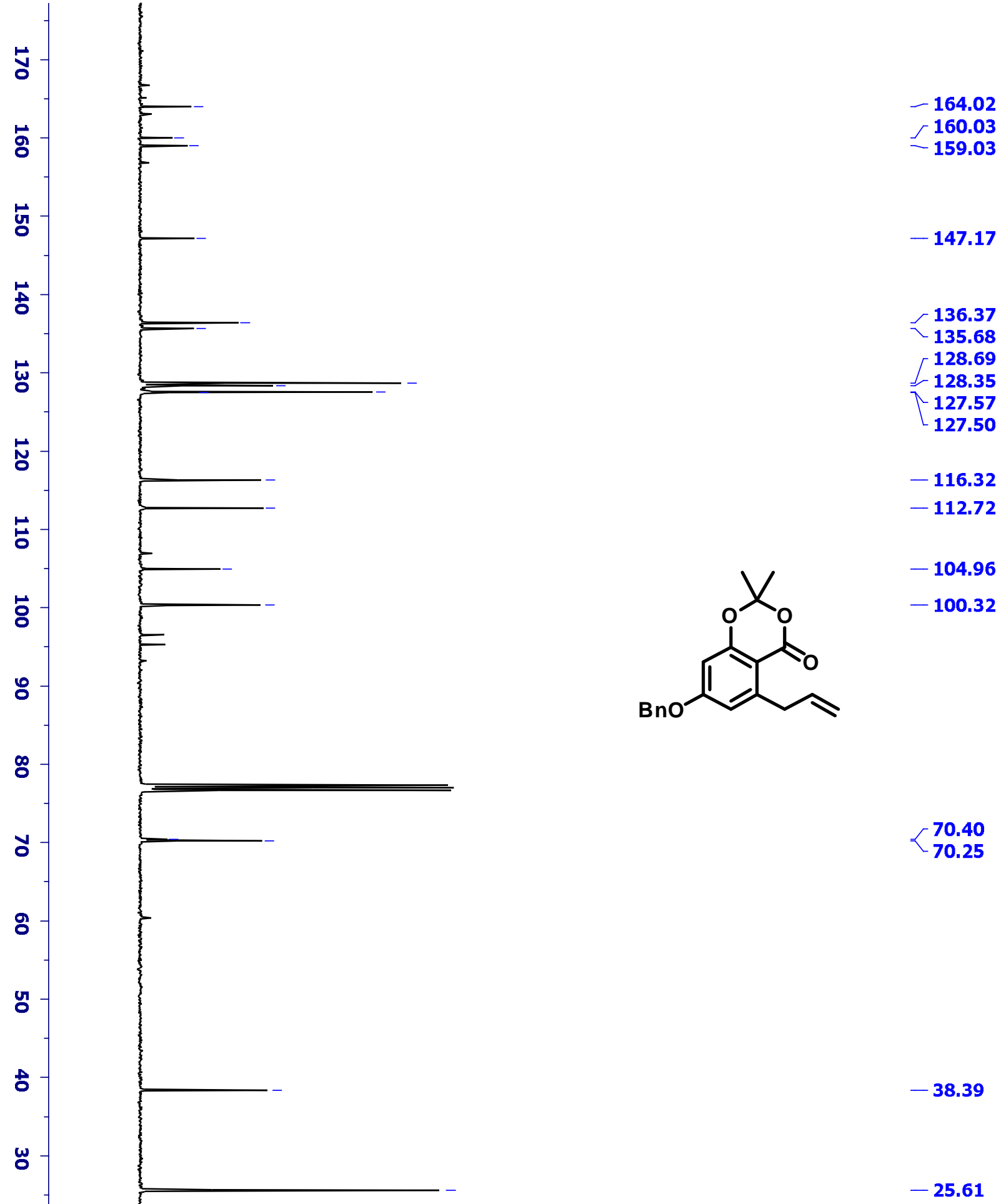

N

능

$\circ$ 


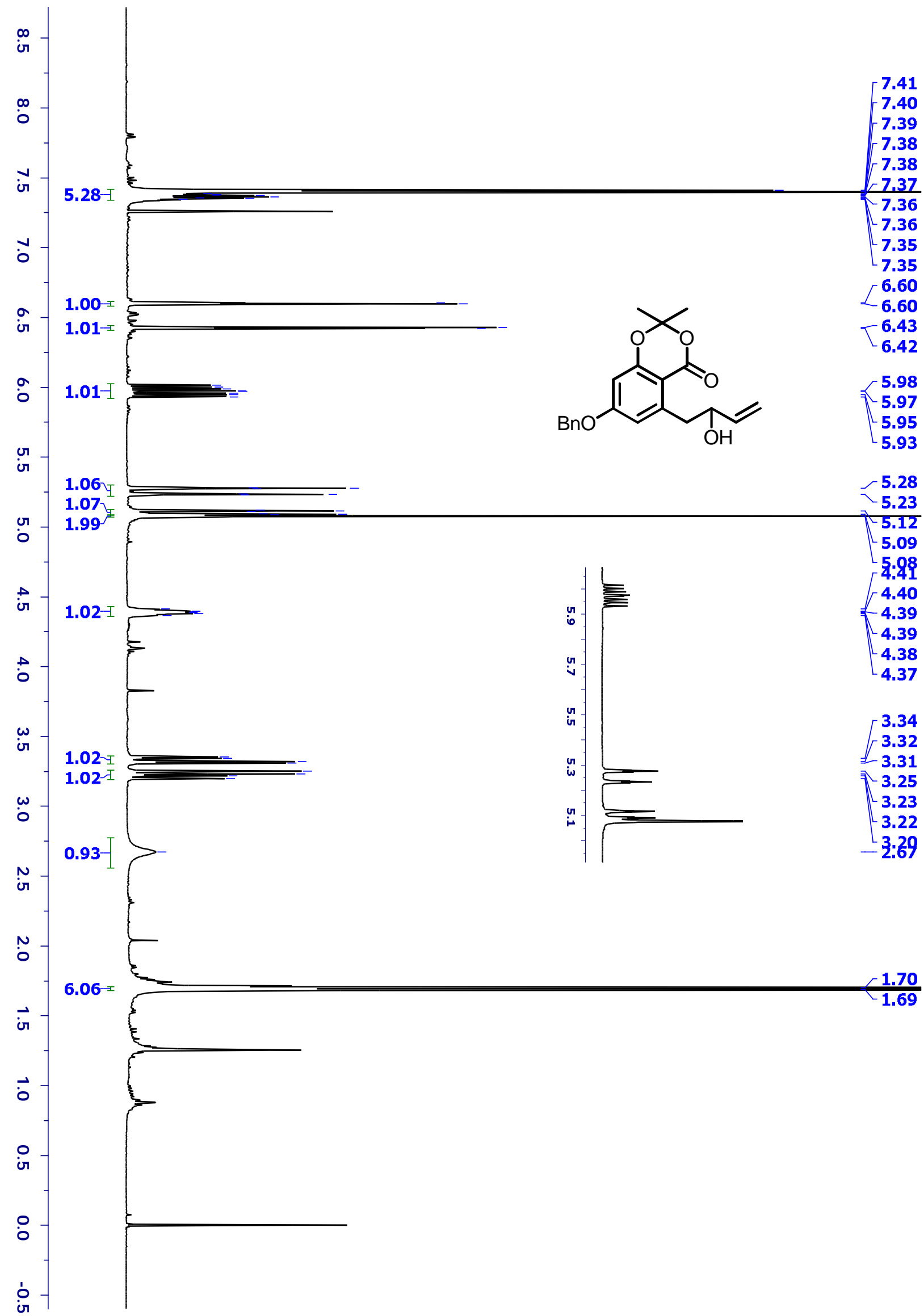

${ }^{1} \mathrm{H}$ NMR spectrum of $21\left(400 \mathrm{MHz}, \mathrm{CDCl}_{3}\right)$ 


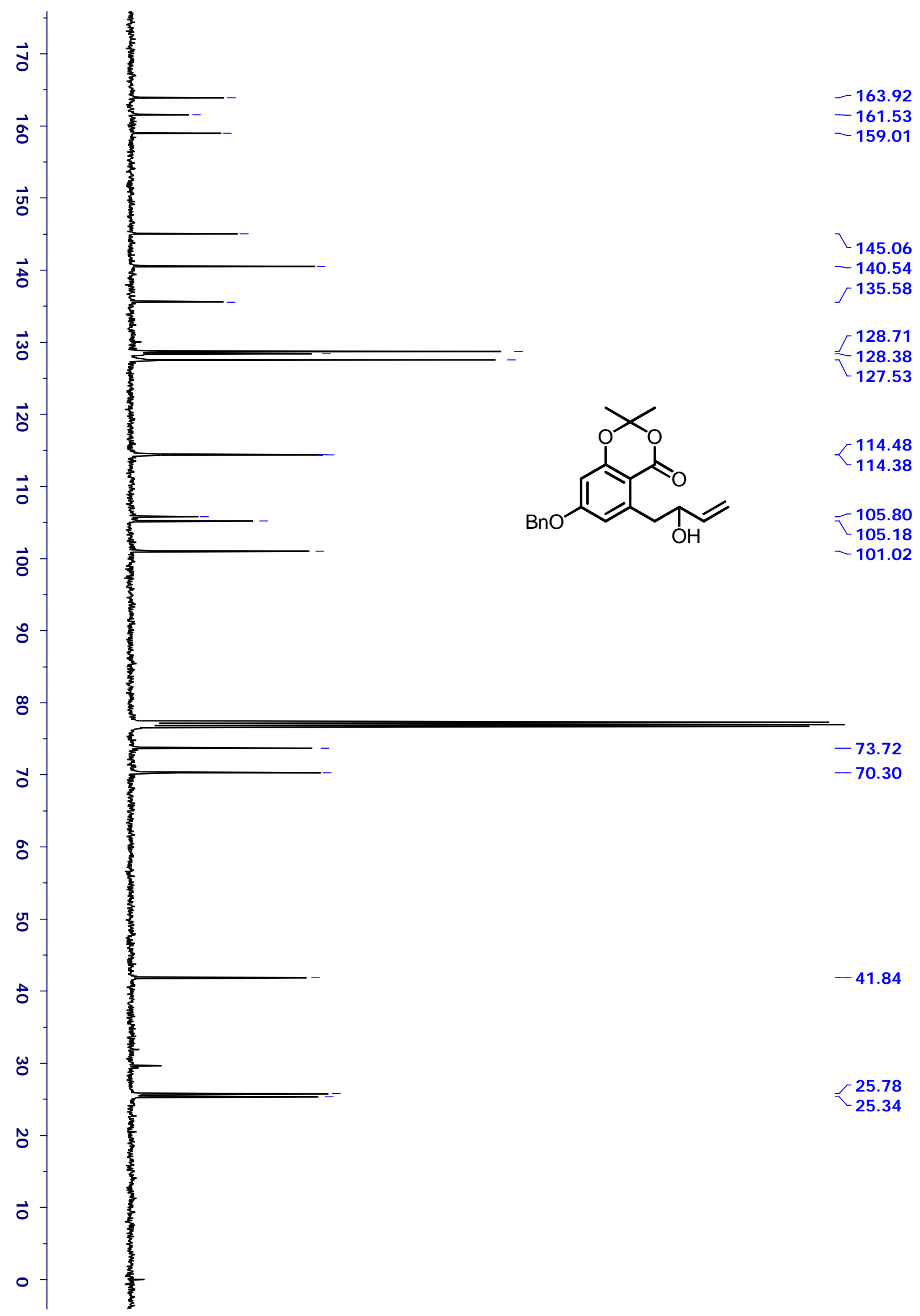

${ }^{13} \mathrm{C}$ NMR spectrum of $21\left(100 \mathrm{MHz}, \mathrm{CDCl}_{3}\right)$ 


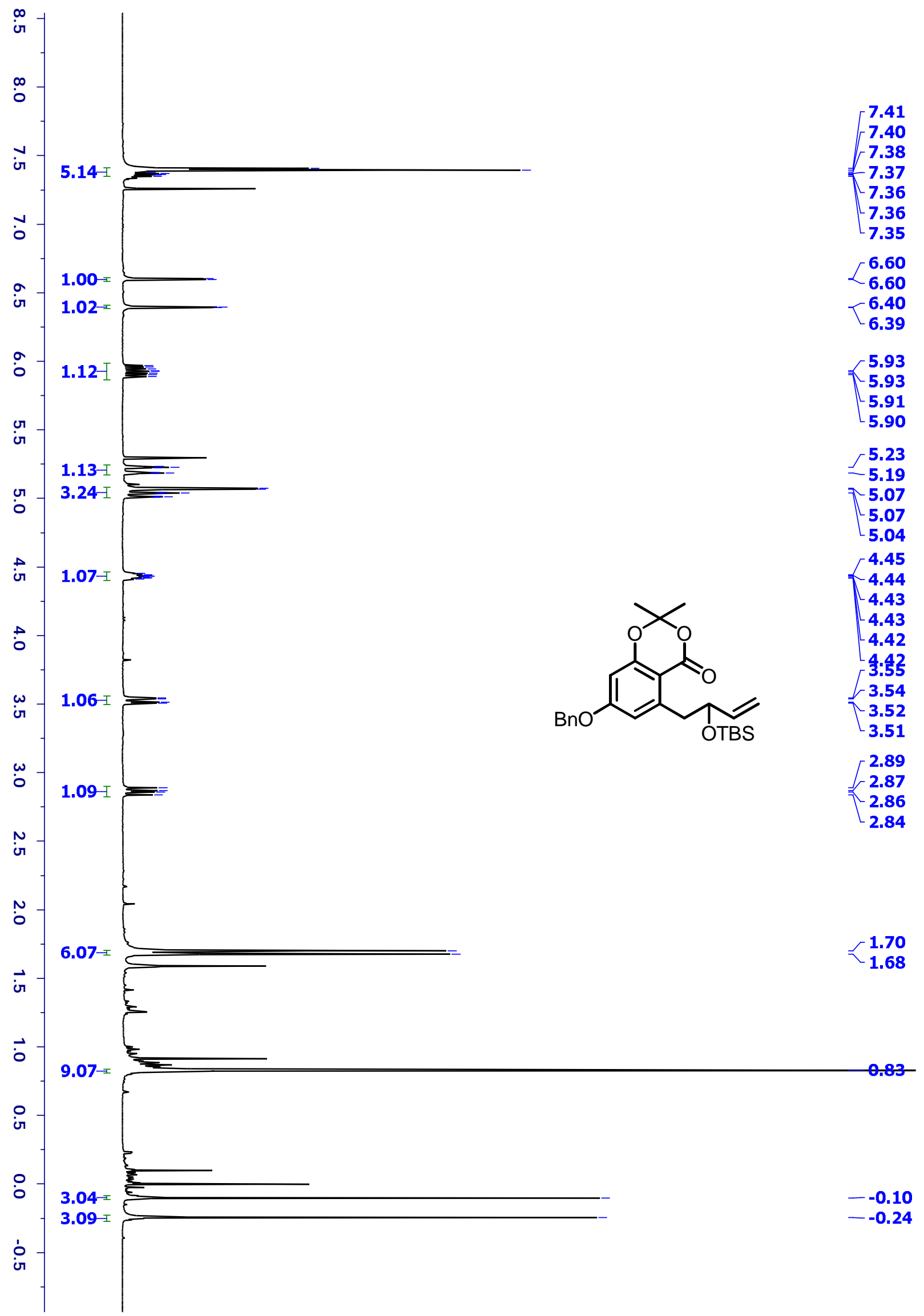

${ }^{1} \mathrm{H}$ NMR spectrum of $\mathbf{1 0}\left(400 \mathrm{MHz}, \mathrm{CDCl}_{3}\right)$ 


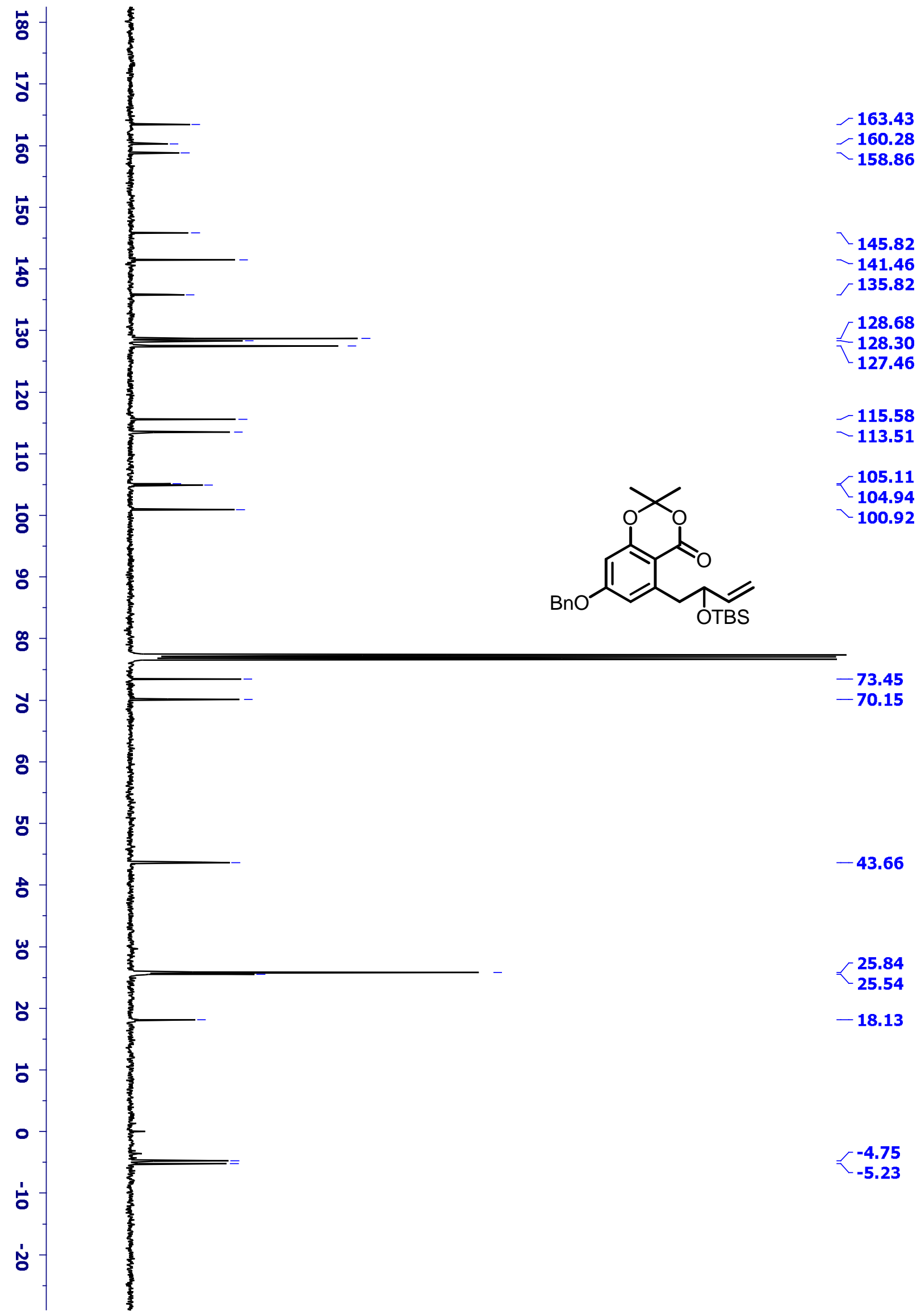

${ }^{13} \mathrm{C}$ NMR spectrum of $\mathbf{1 0}\left(100 \mathrm{MHz}, \mathrm{CDCl}_{3}\right)$ 


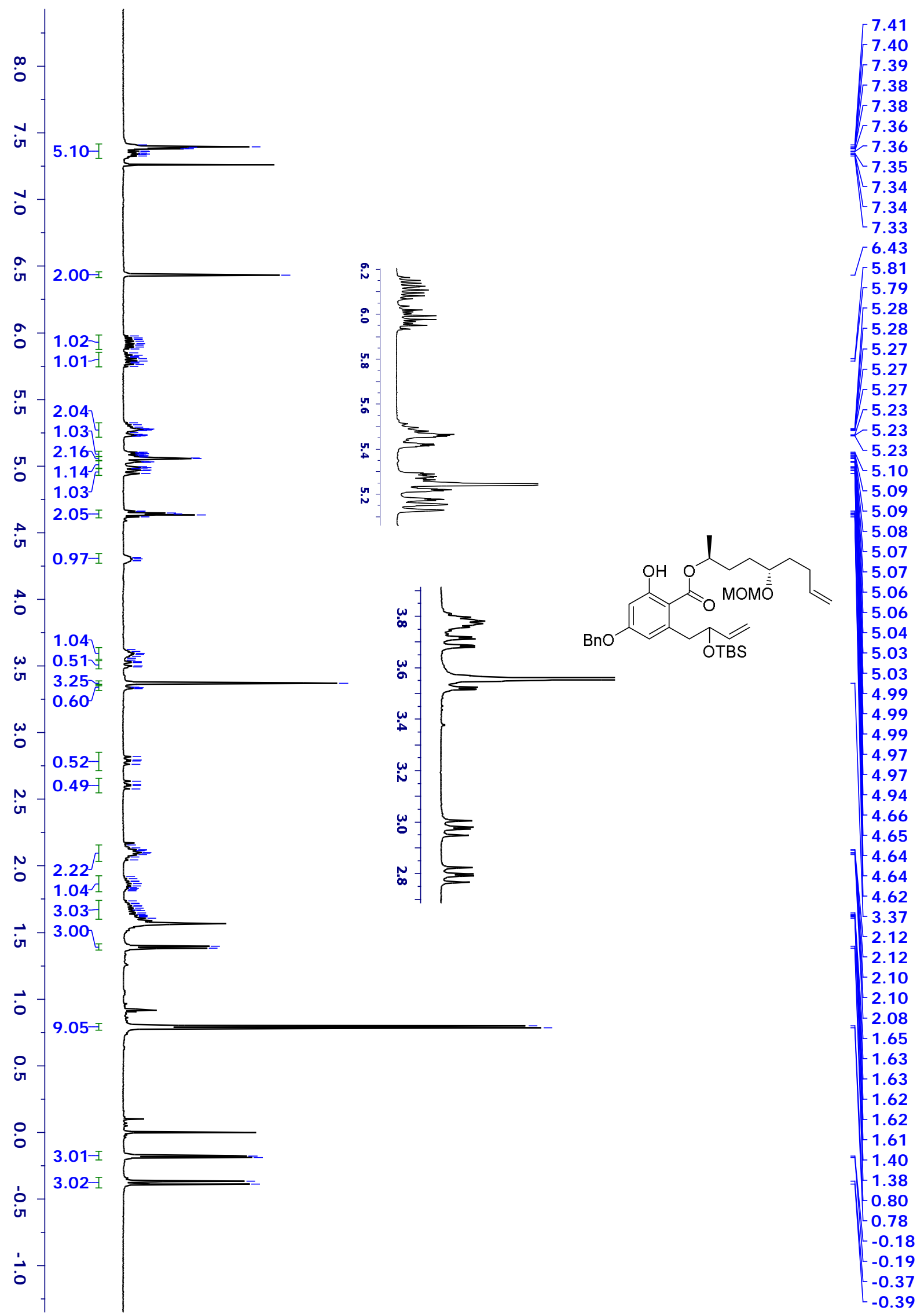

${ }^{1} \mathrm{H}$ NMR spectrum of $22\left(400 \mathrm{MHz}, \mathrm{CDCl}_{3}\right)$ 


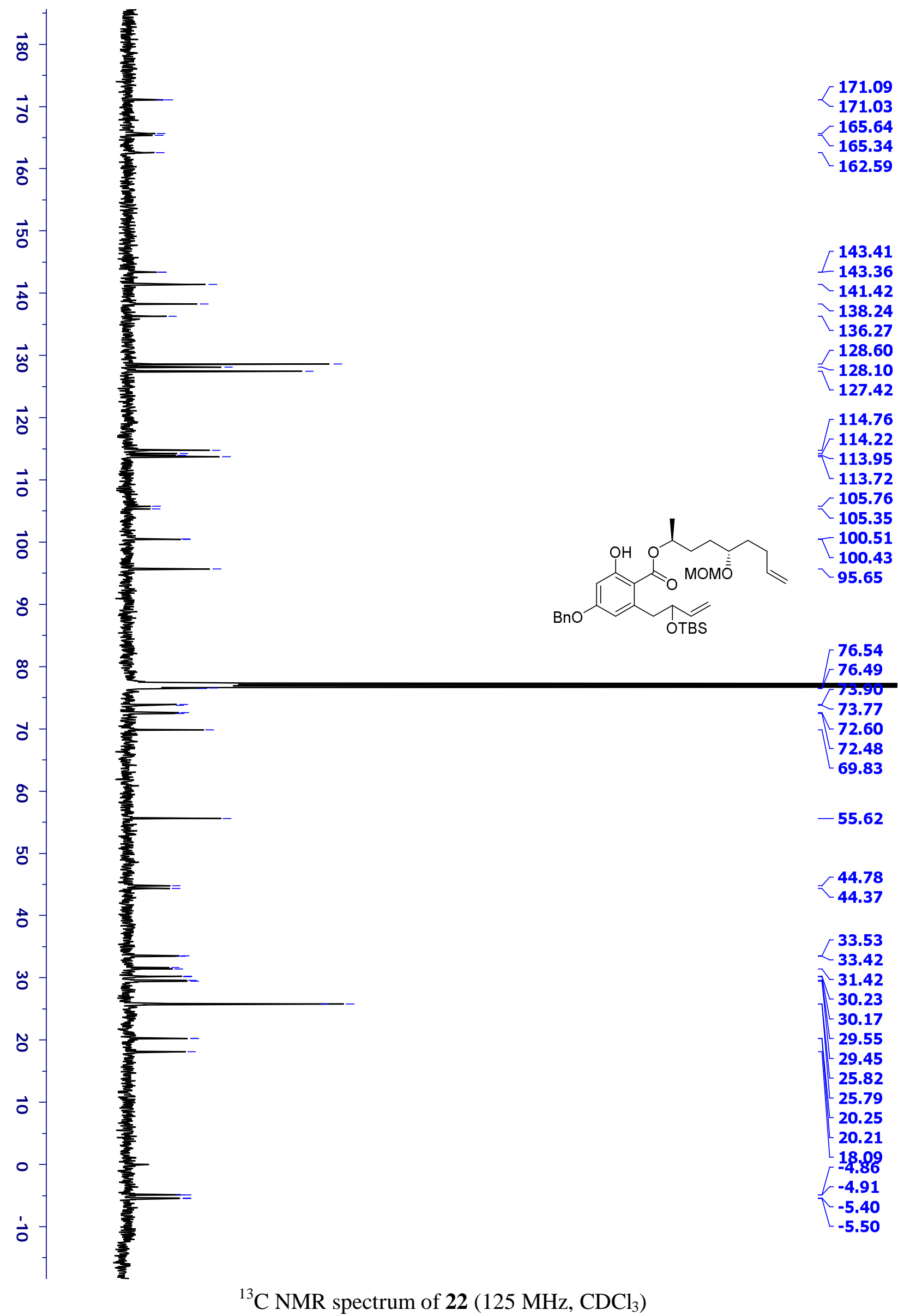




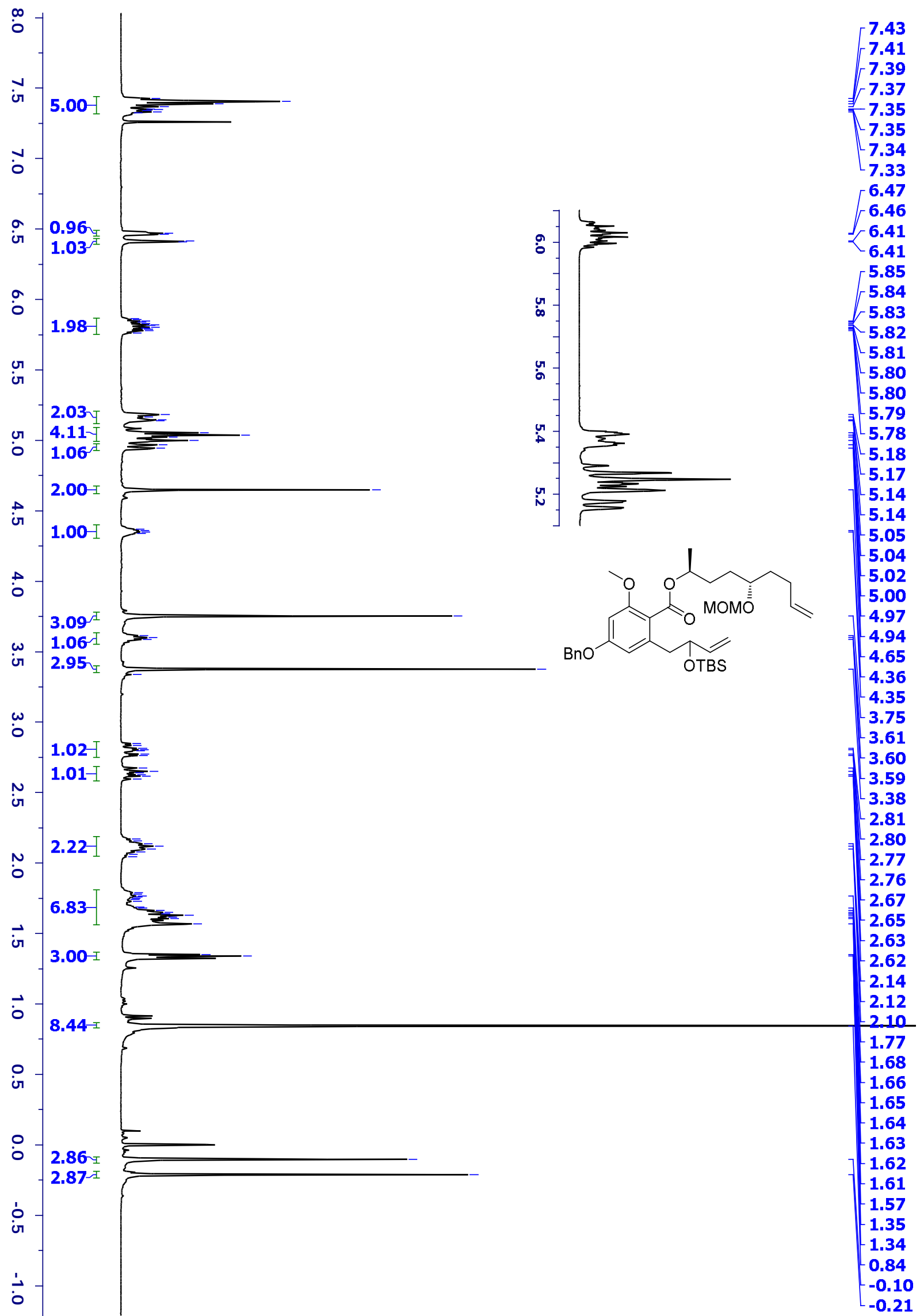

${ }^{1} \mathrm{H}$ NMR spectrum of $23\left(400 \mathrm{MHz}, \mathrm{CDCl}_{3}\right)$ 


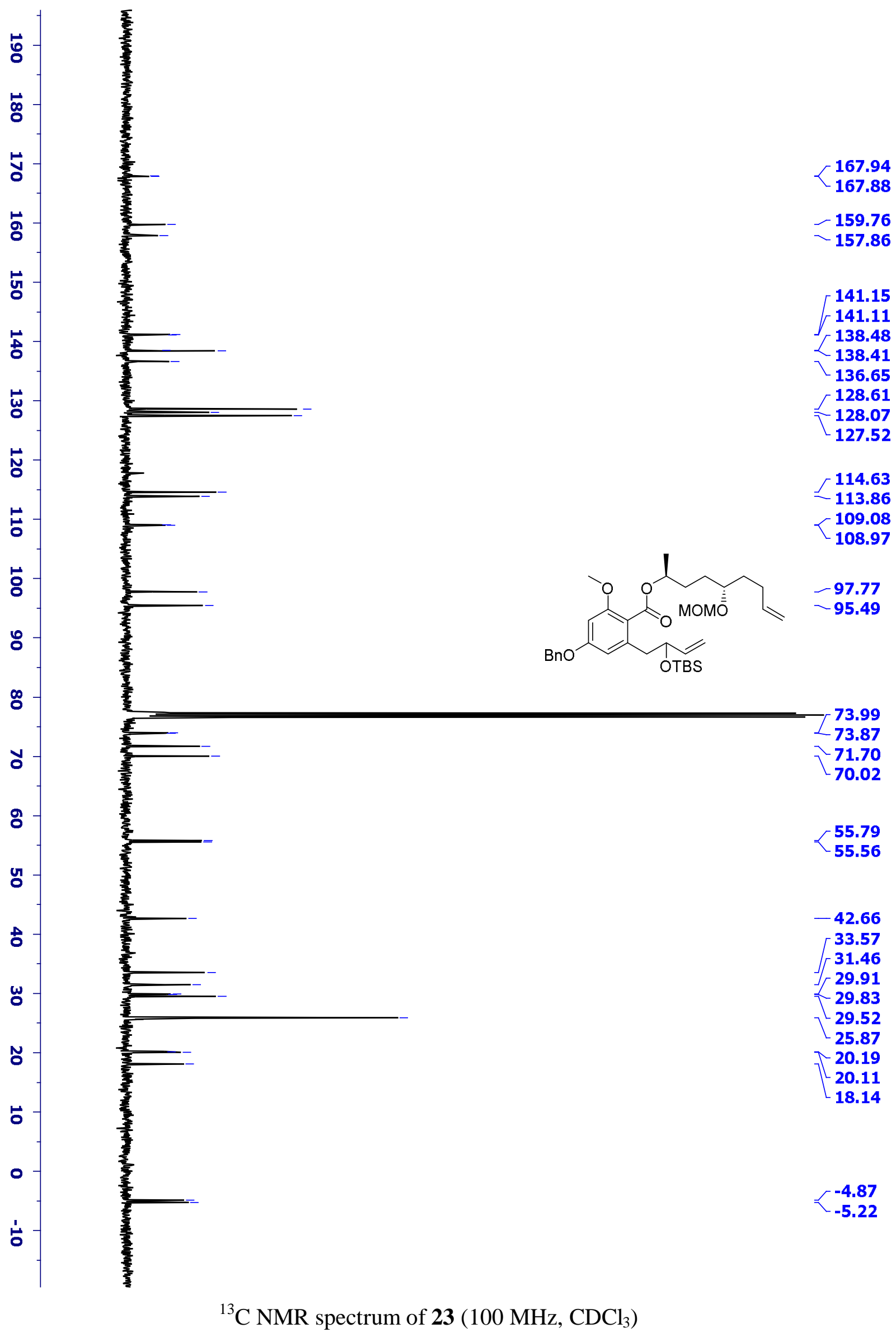




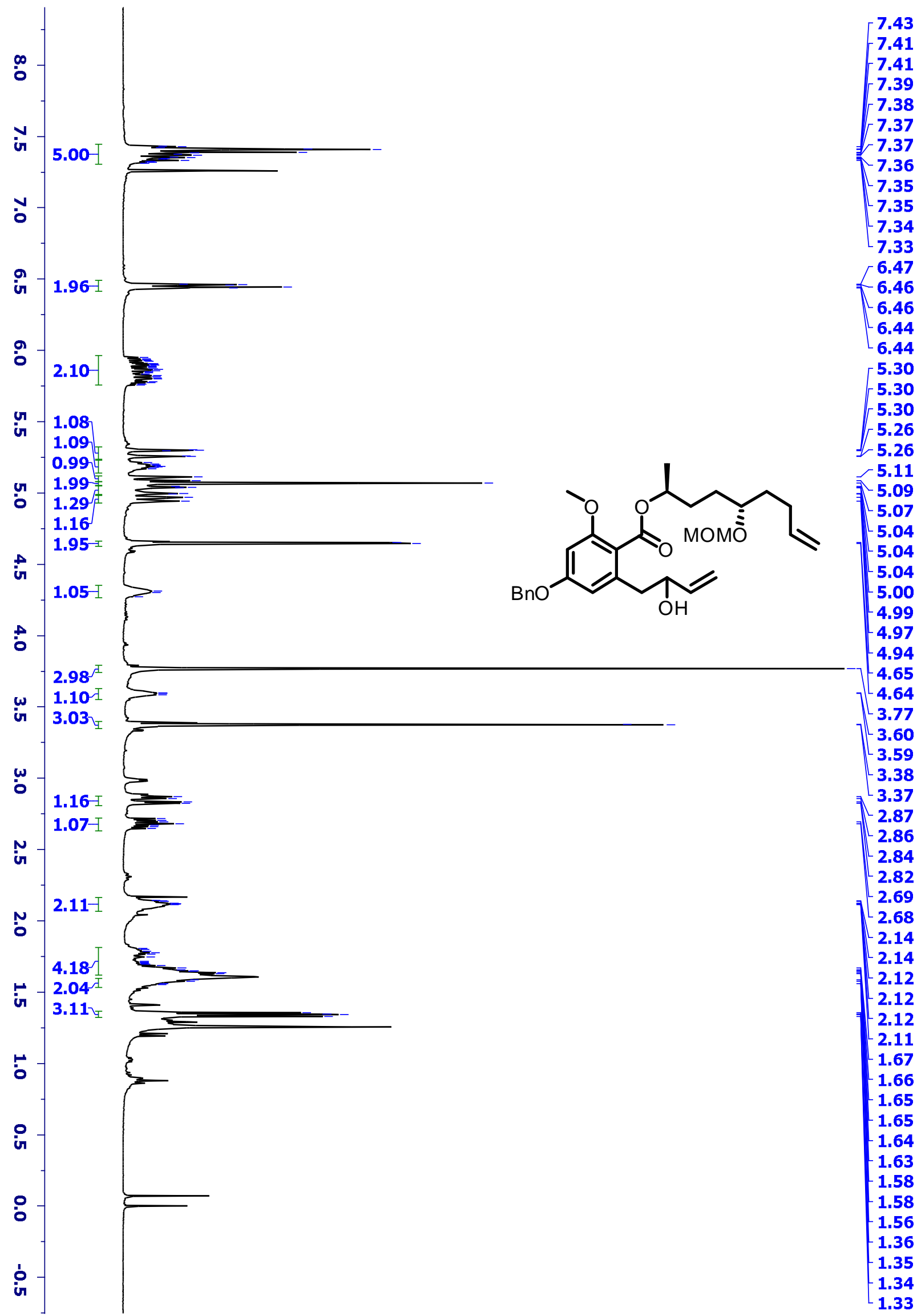

${ }^{1} \mathrm{H}$ NMR spectrum of $24\left(400 \mathrm{MHz}, \mathrm{CDCl}_{3}\right)$ 


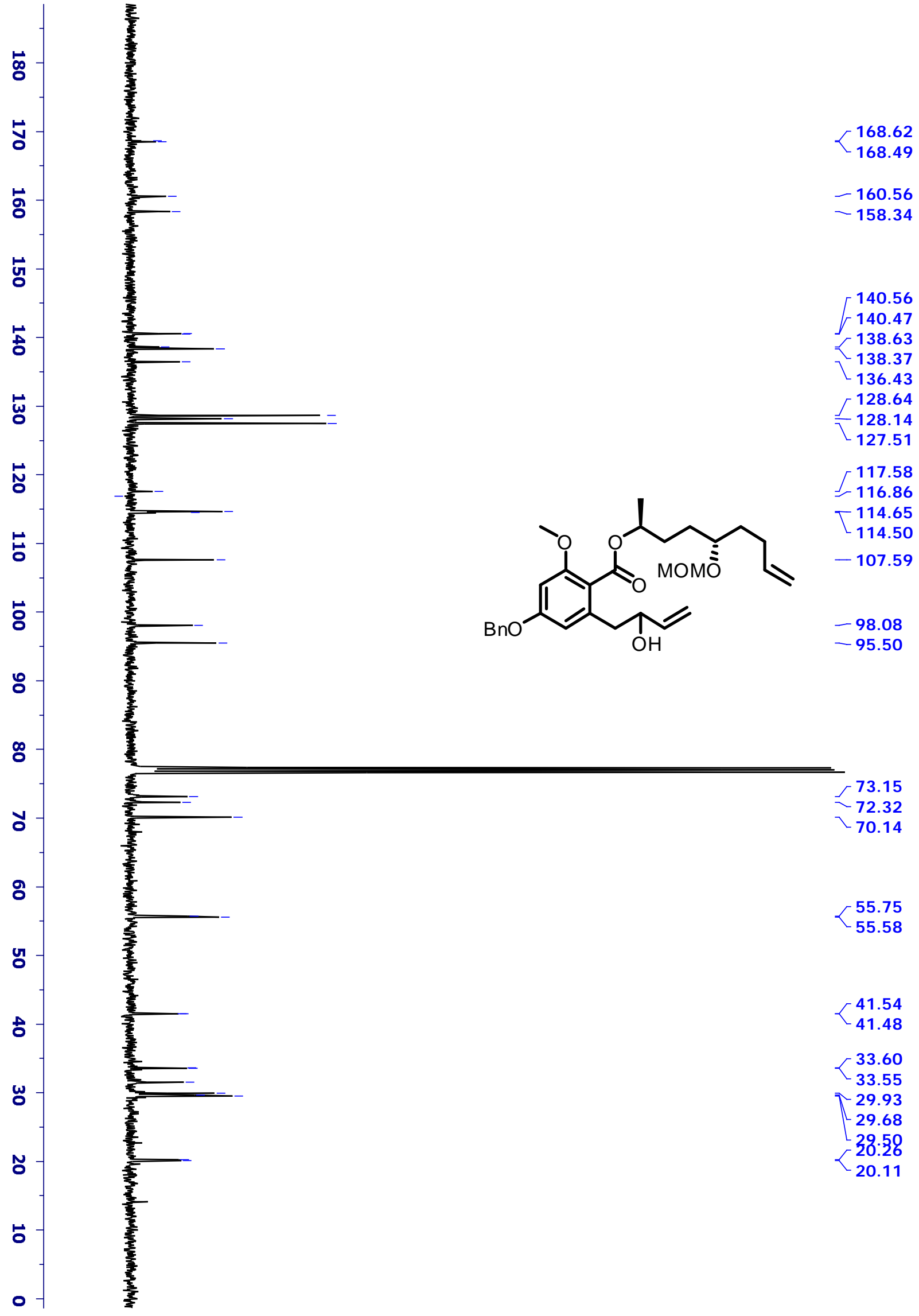

${ }^{13} \mathrm{C}$ NMR spectrum of $24\left(100 \mathrm{MHz}, \mathrm{CDCl}_{3}\right)$ 


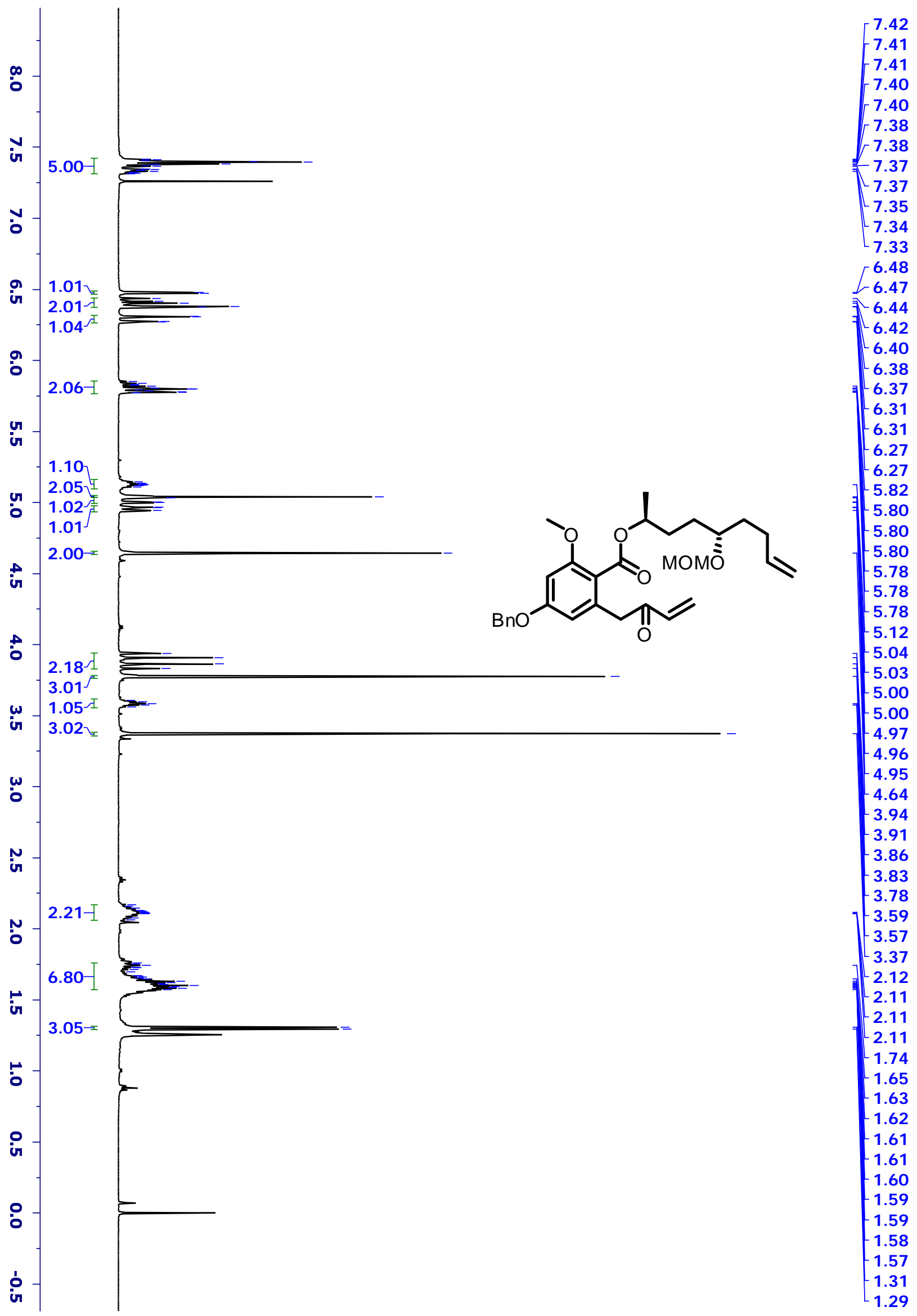

${ }^{1} \mathrm{H}$ NMR spectrum of $9\left(500 \mathrm{MHz}, \mathrm{CDCl}_{3}\right)$ 


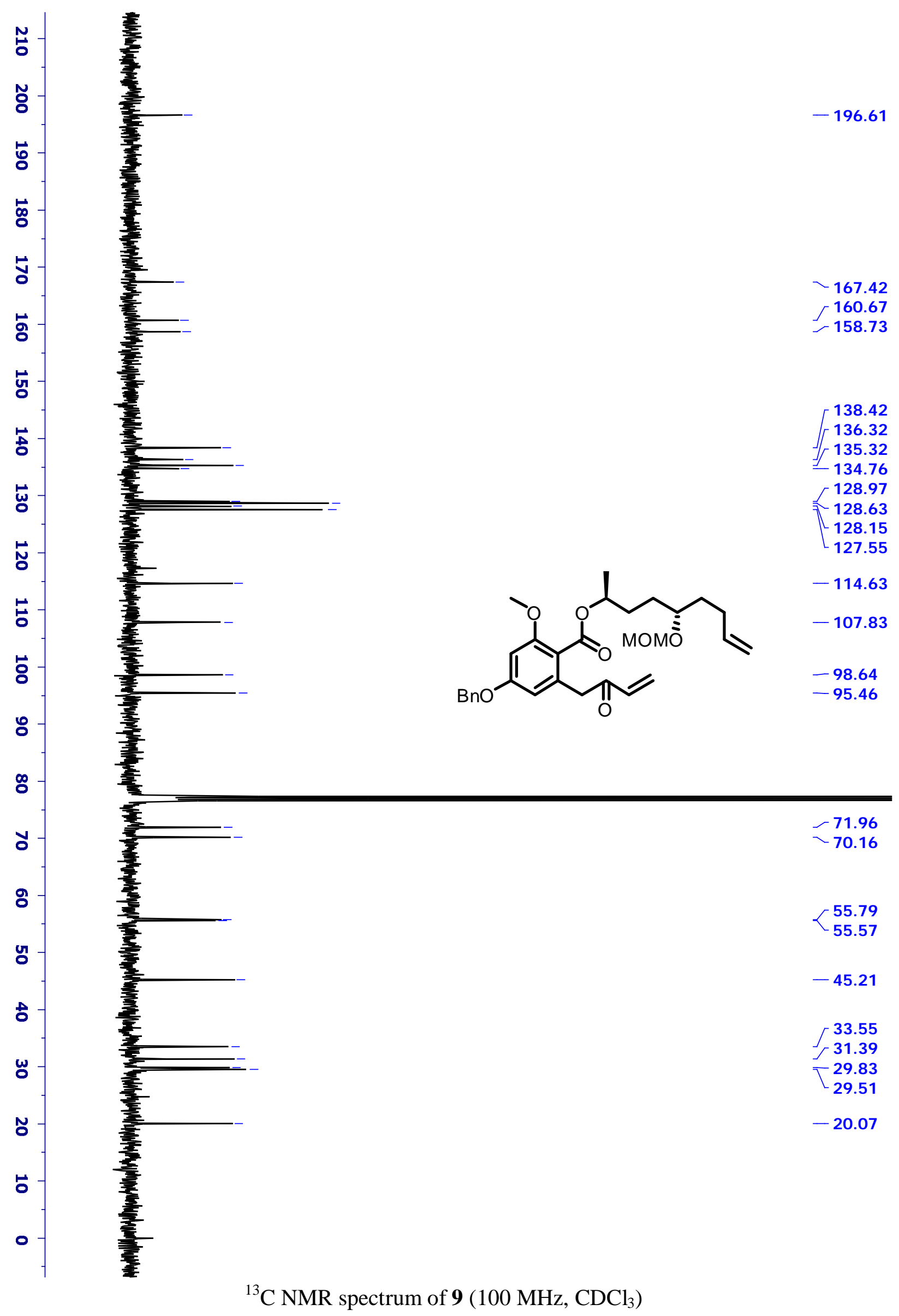




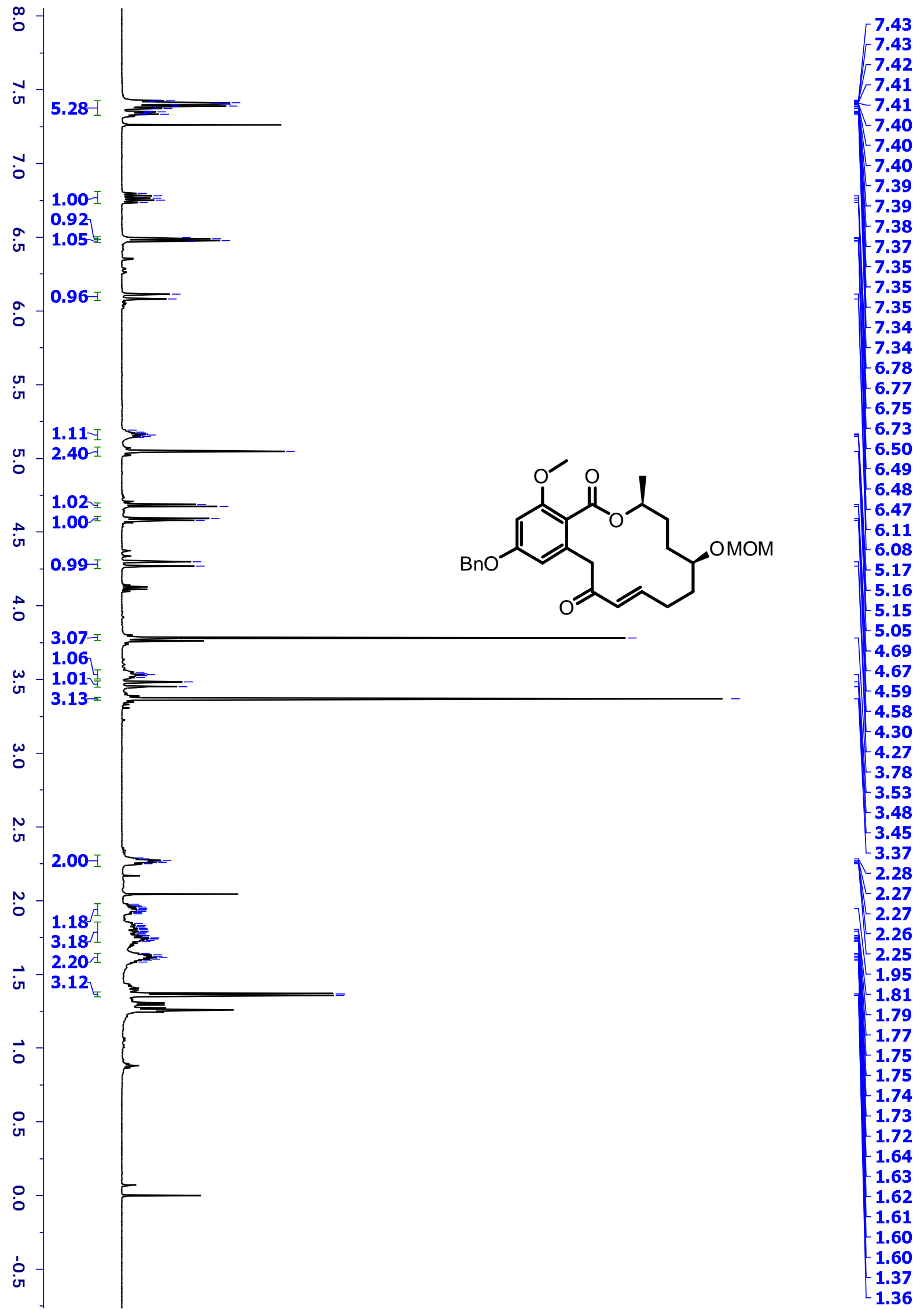

${ }^{1} \mathrm{H}$ NMR spectrum of $7\left(500 \mathrm{MHz}, \mathrm{CDCl}_{3}\right)$ 


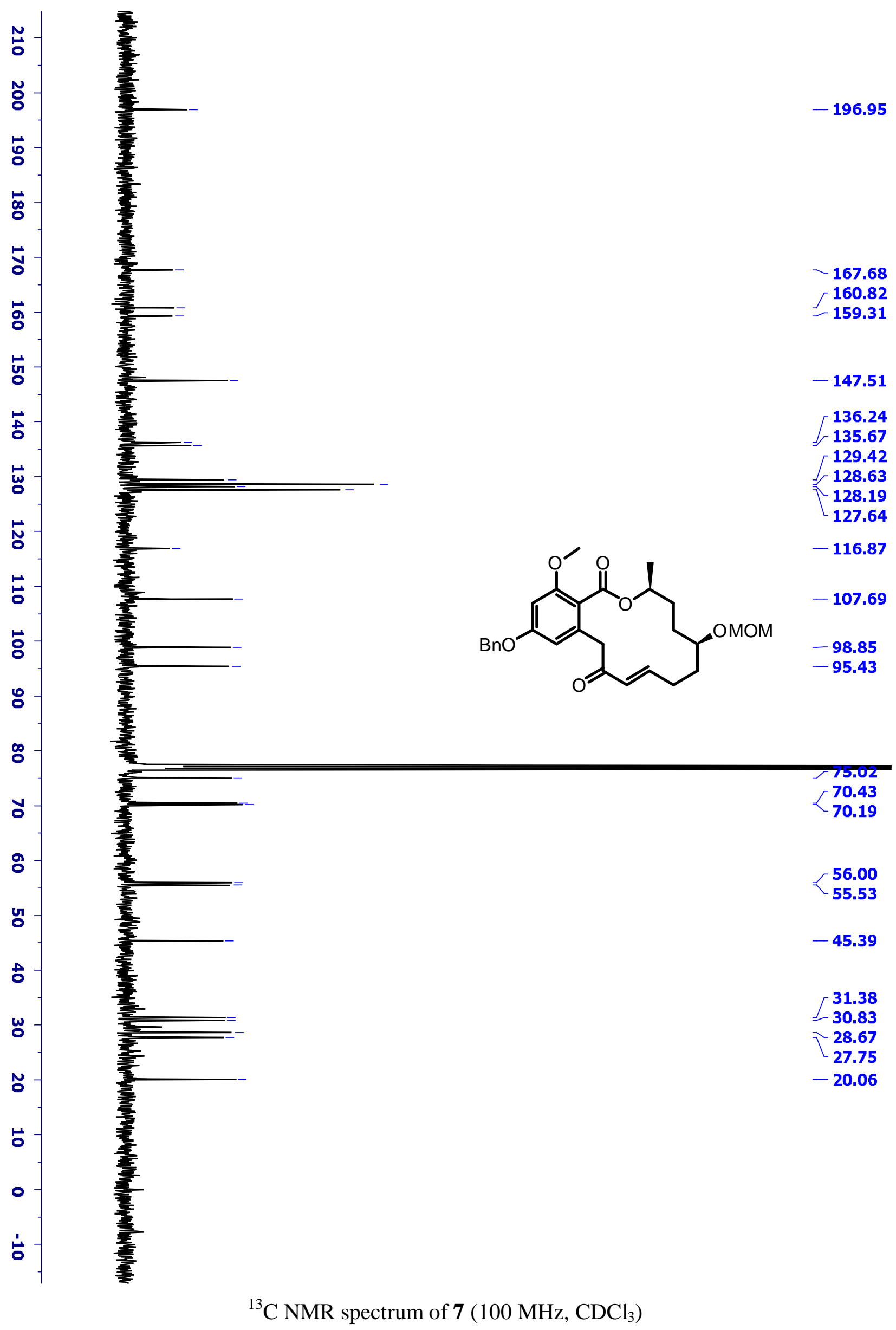



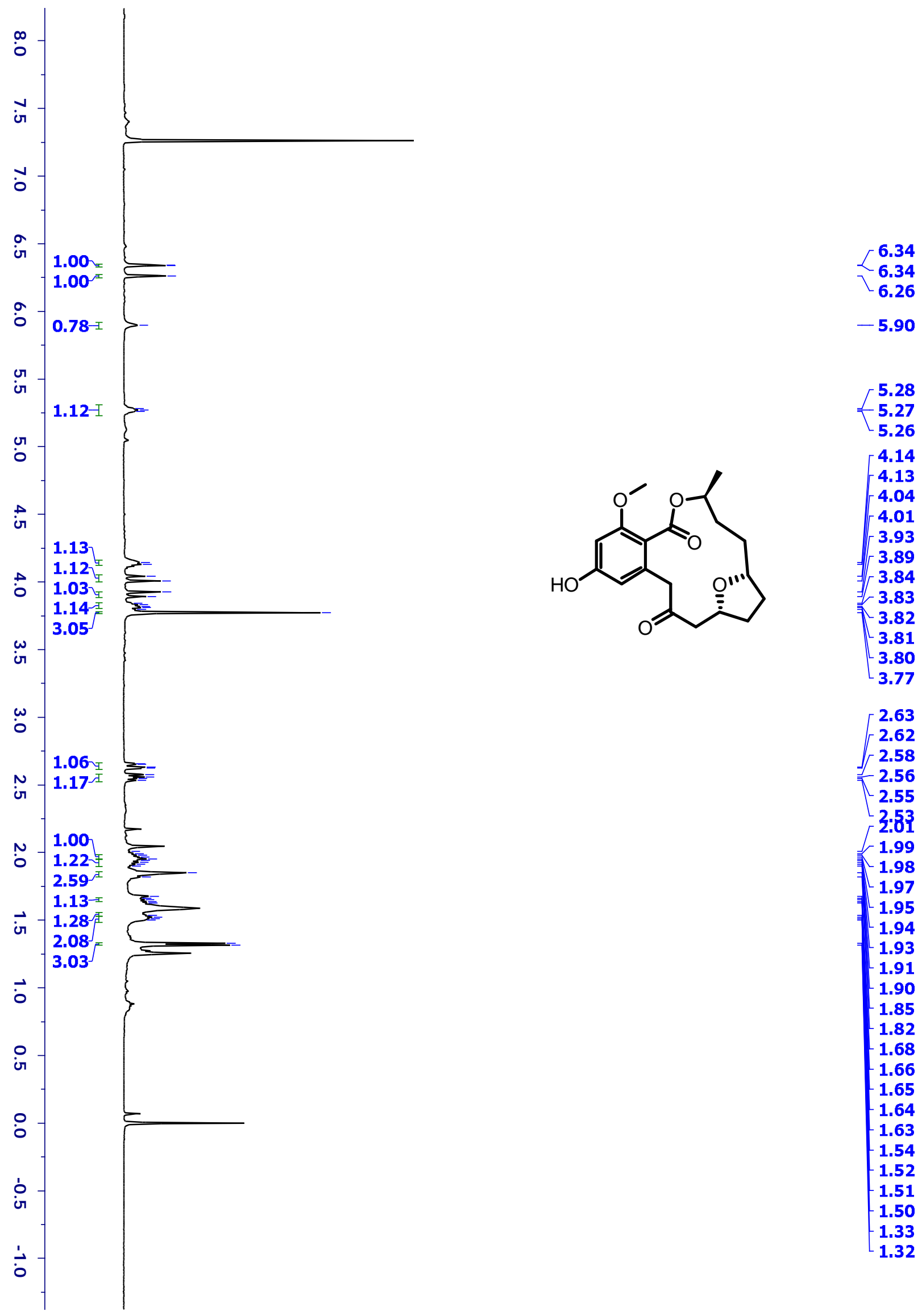

5.28

5.27

5.26

4.14

4.13

4.04

4.01

3.93

3.89

3.84

3.83

3.82

3.81

3.80

3.77

2.63

2.62

2.58

$-2.56$

2.55

2:53

1.99

1.98

1.97

1.95

1.94

1.93

1.91

1.90

1.85

1.82

1.68

1.66

1.65

1.64

1.63

1.54

$-1.52$

1.51

1.50

1.33

${ }^{1} \mathrm{H}$ NMR spectrum of $4 \mathbf{a}\left(500 \mathrm{MHz}, \mathrm{CDCl}_{3}\right)$ 


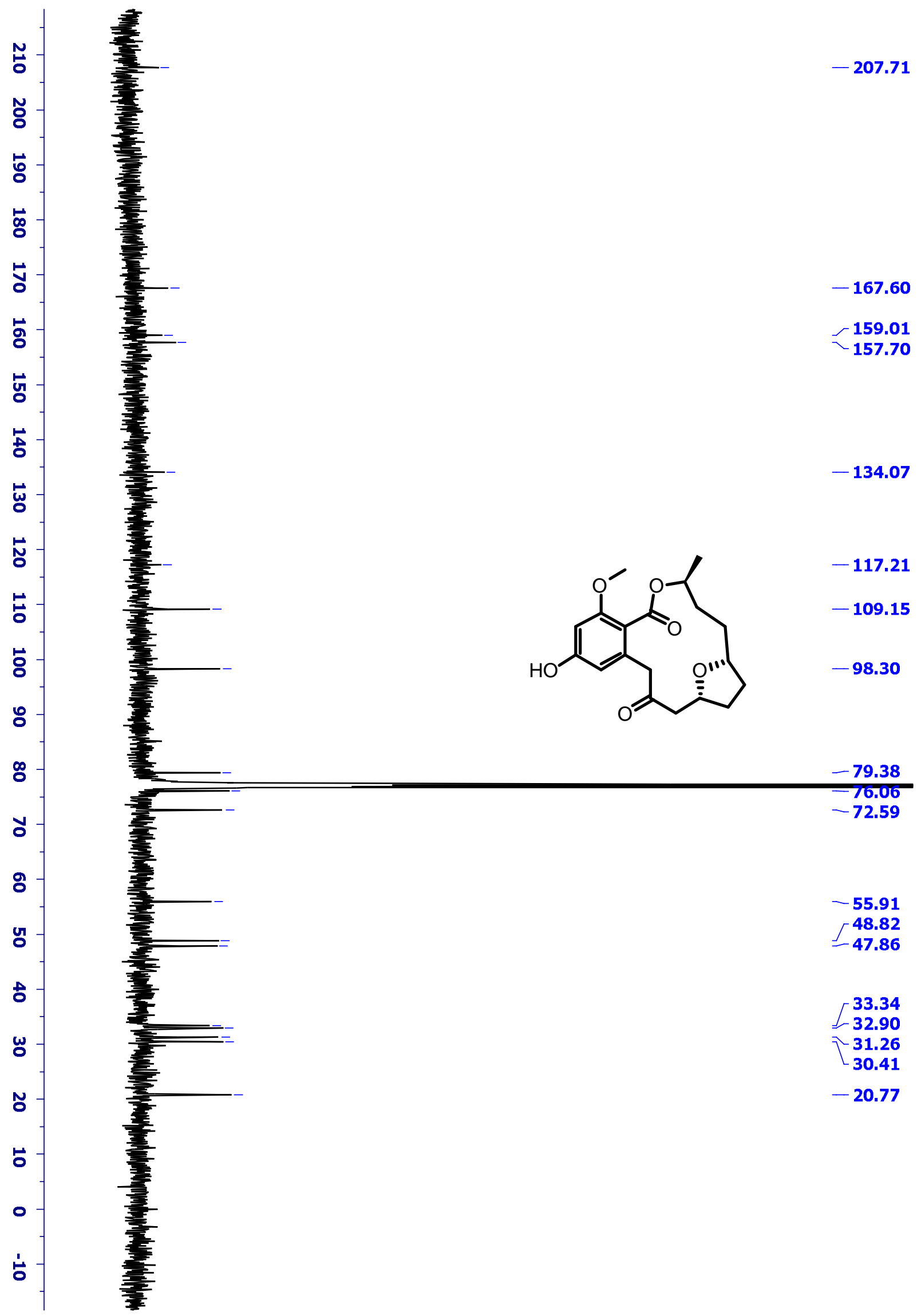

${ }^{13} \mathrm{C}$ NMR spectrum of $\mathbf{4 a}\left(125 \mathrm{MHz}, \mathrm{CDCl}_{3}\right)$ 


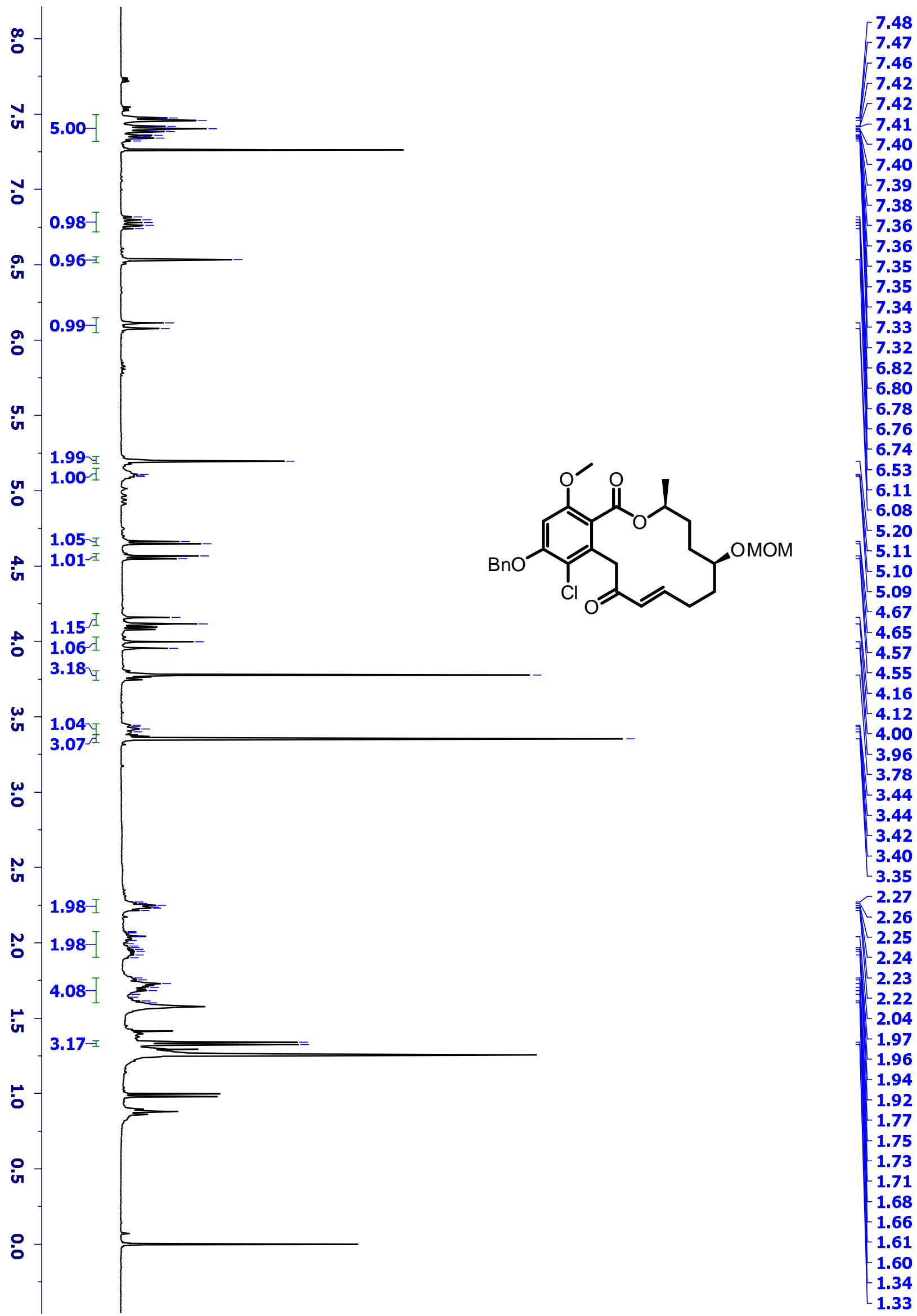

${ }^{1} \mathrm{H}$ NMR spectrum of $8\left(400 \mathrm{MHz}, \mathrm{CDCl}_{3}\right)$ 


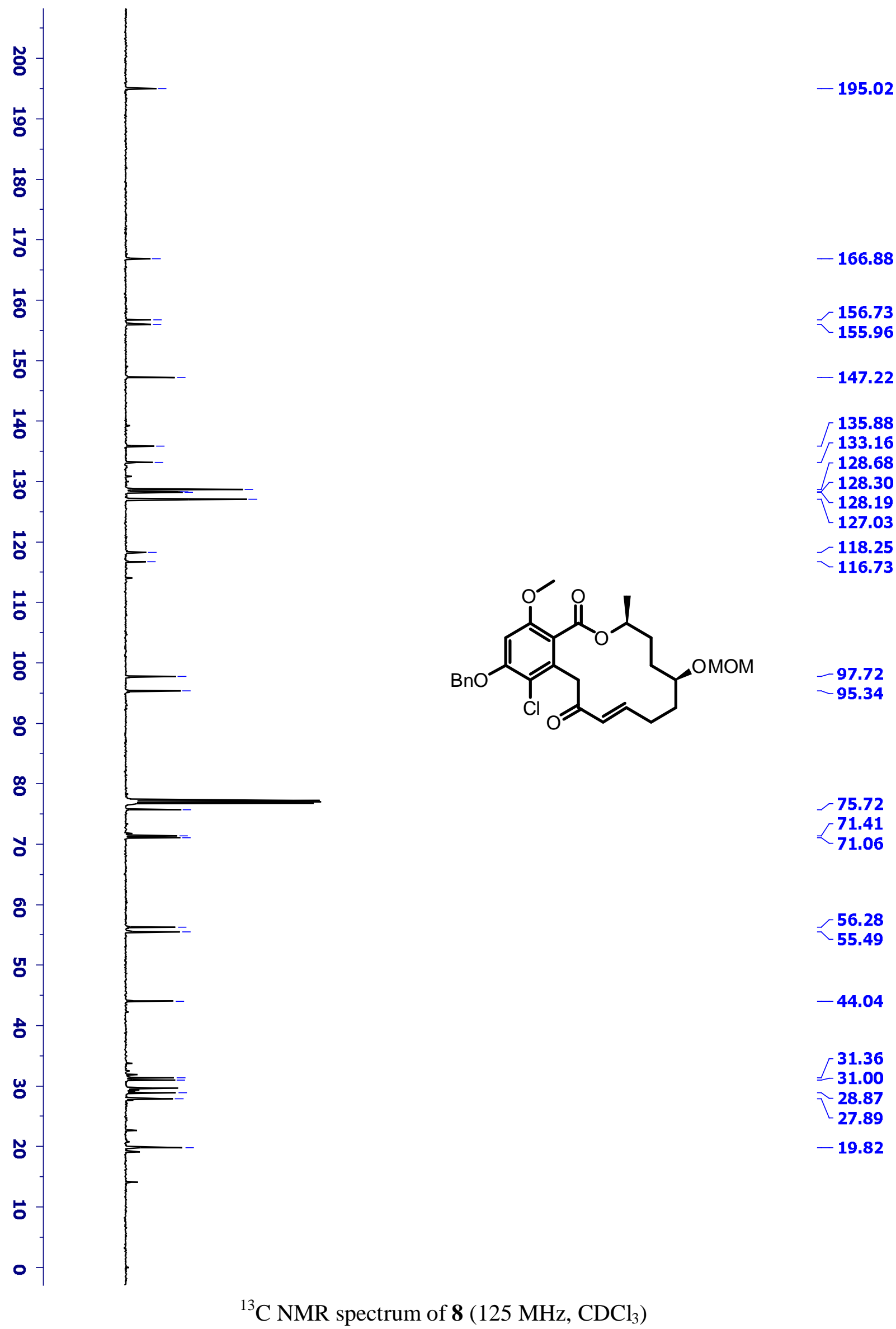



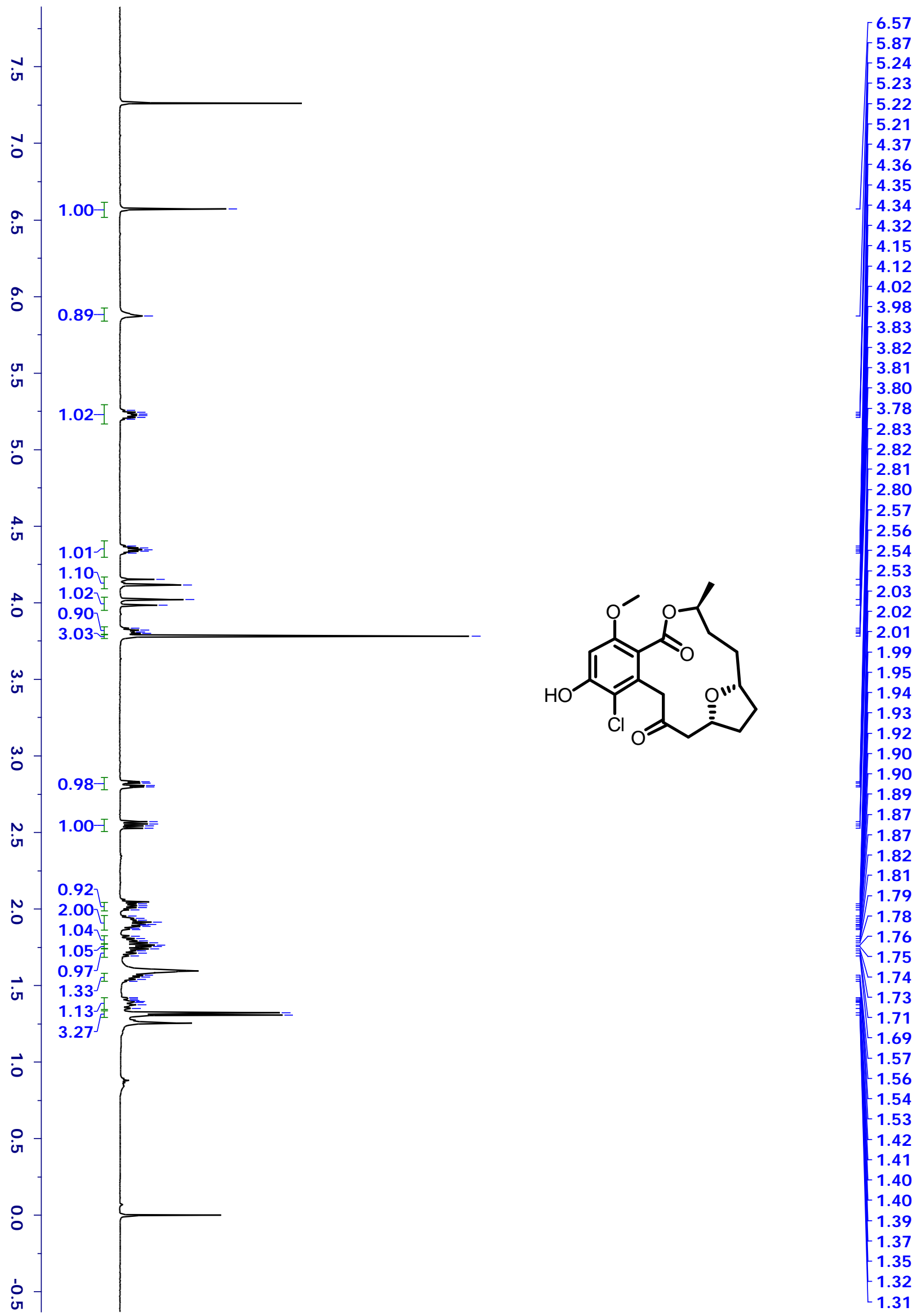

${ }^{1} \mathrm{H}$ NMR spectrum of $\mathbf{5 a}\left(400 \mathrm{MHz}, \mathrm{CDCl}_{3}\right)$ 


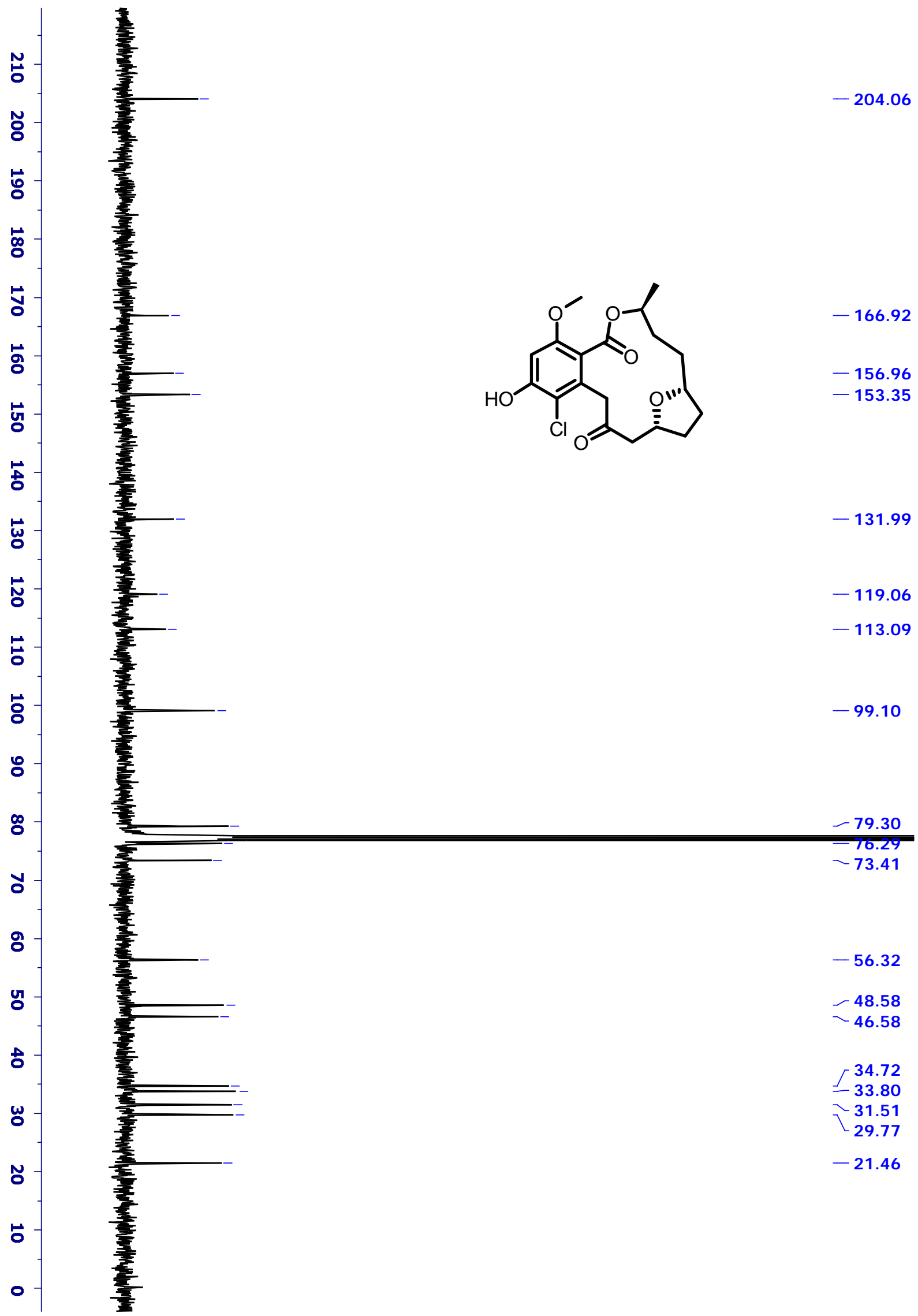

${ }^{13} \mathrm{C}$ NMR spectrum of $\mathbf{5 a}\left(100 \mathrm{MHz}, \mathrm{CDCl}_{3}\right)$ 


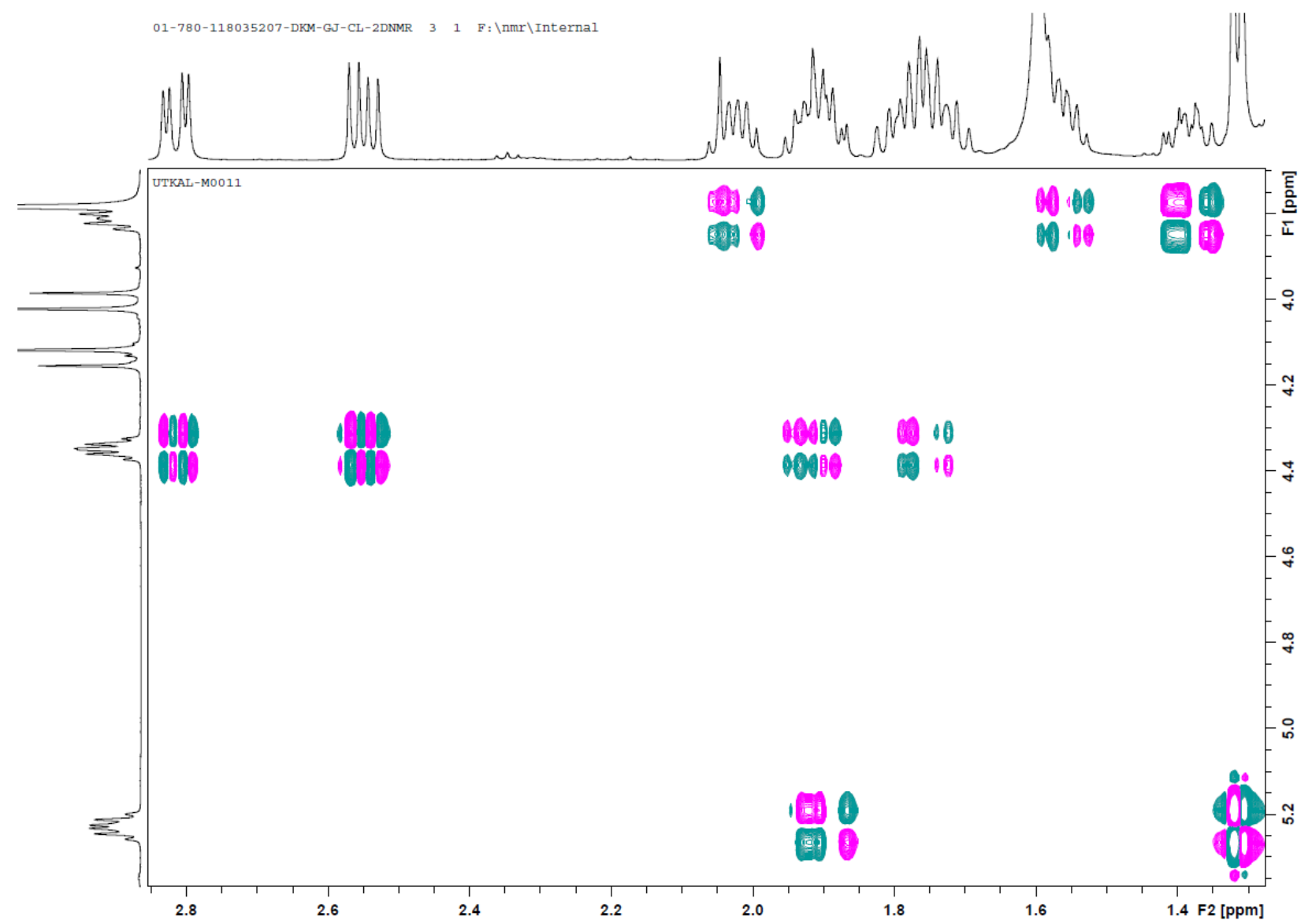

Exapnded version of the DQF-COSY Spectrum of $\mathbf{5 a}\left(500 \mathrm{MHz}, \mathrm{CDCl}_{3}\right)$

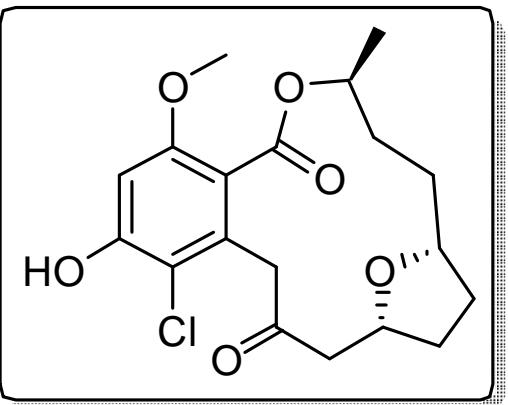




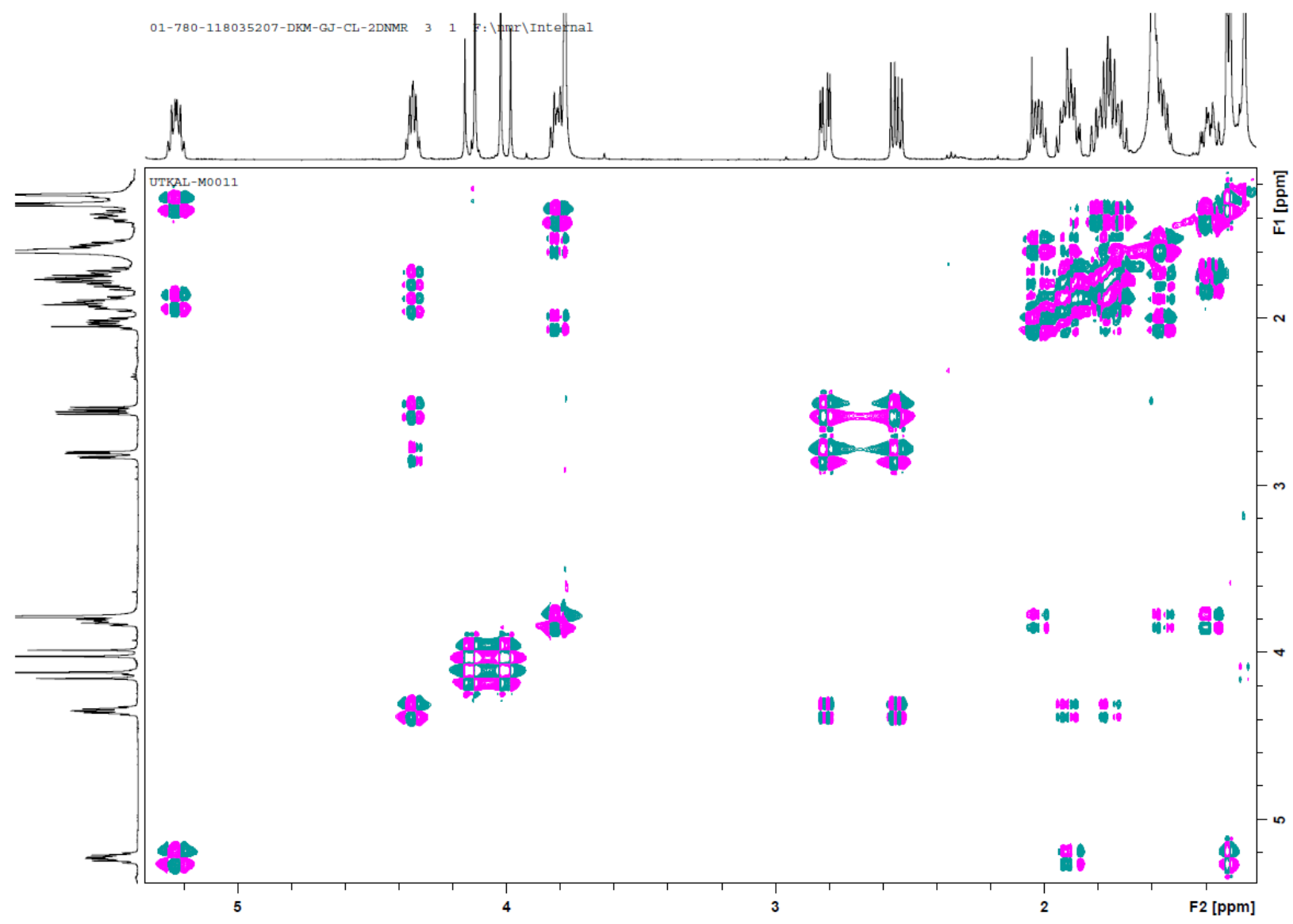

Exapnded version of the DQF-COSY Spectrum of $\mathbf{5 a}\left(500 \mathrm{MHz}, \mathrm{CDCl}_{3}\right)$

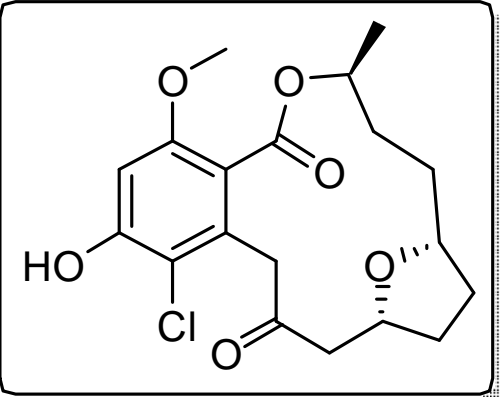




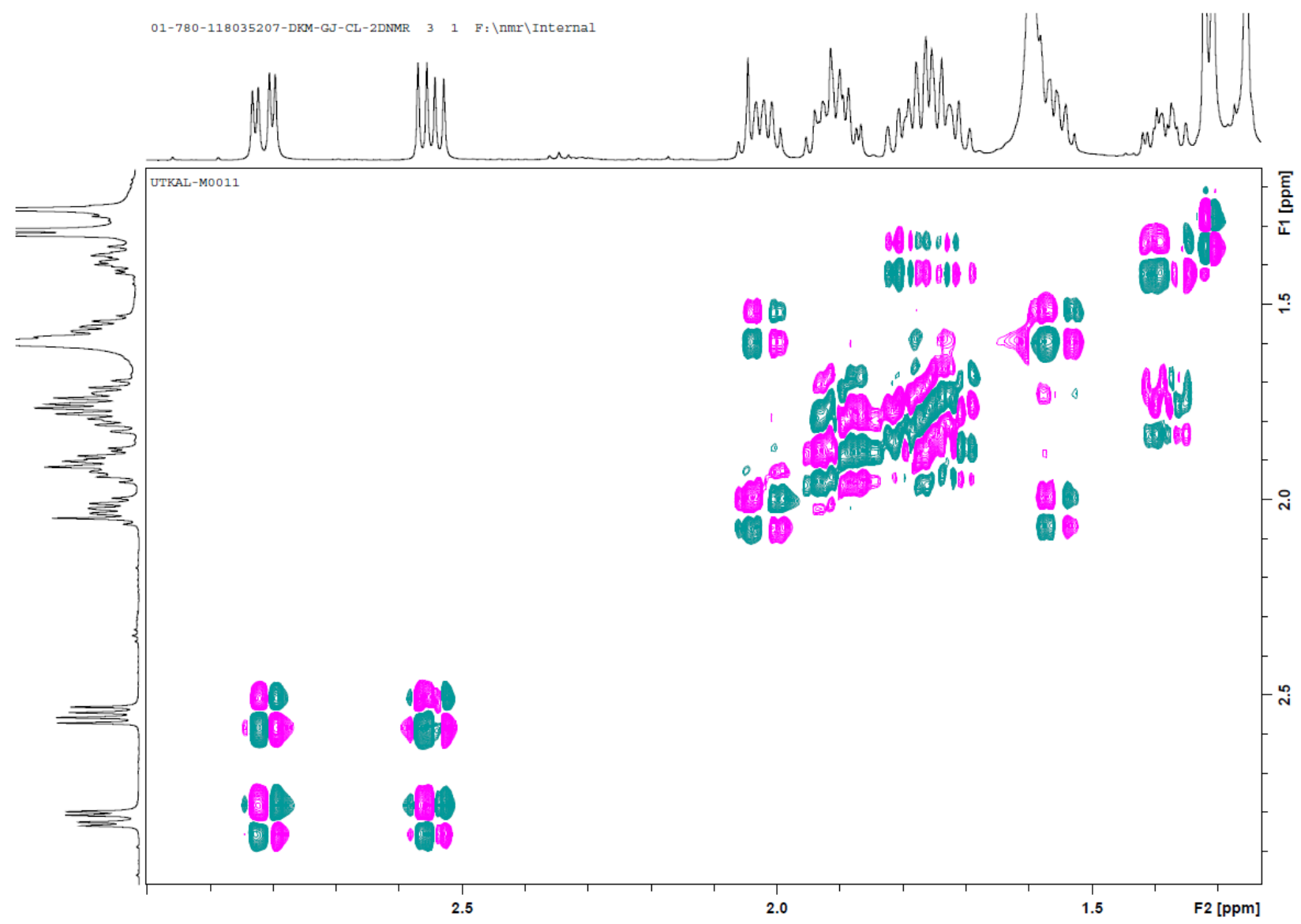

Exapnded version of the DQF-COSY Spectrum of $\mathbf{5 a}\left(500 \mathrm{MHz}, \mathrm{CDCl}_{3}\right)$

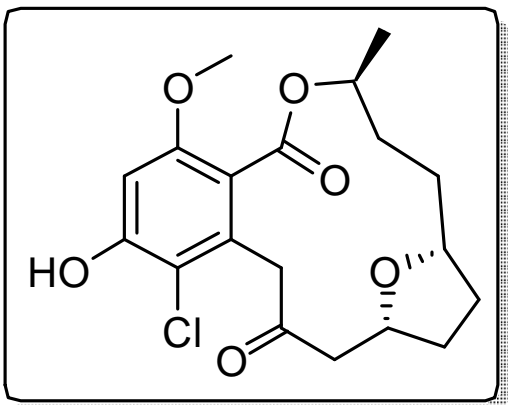




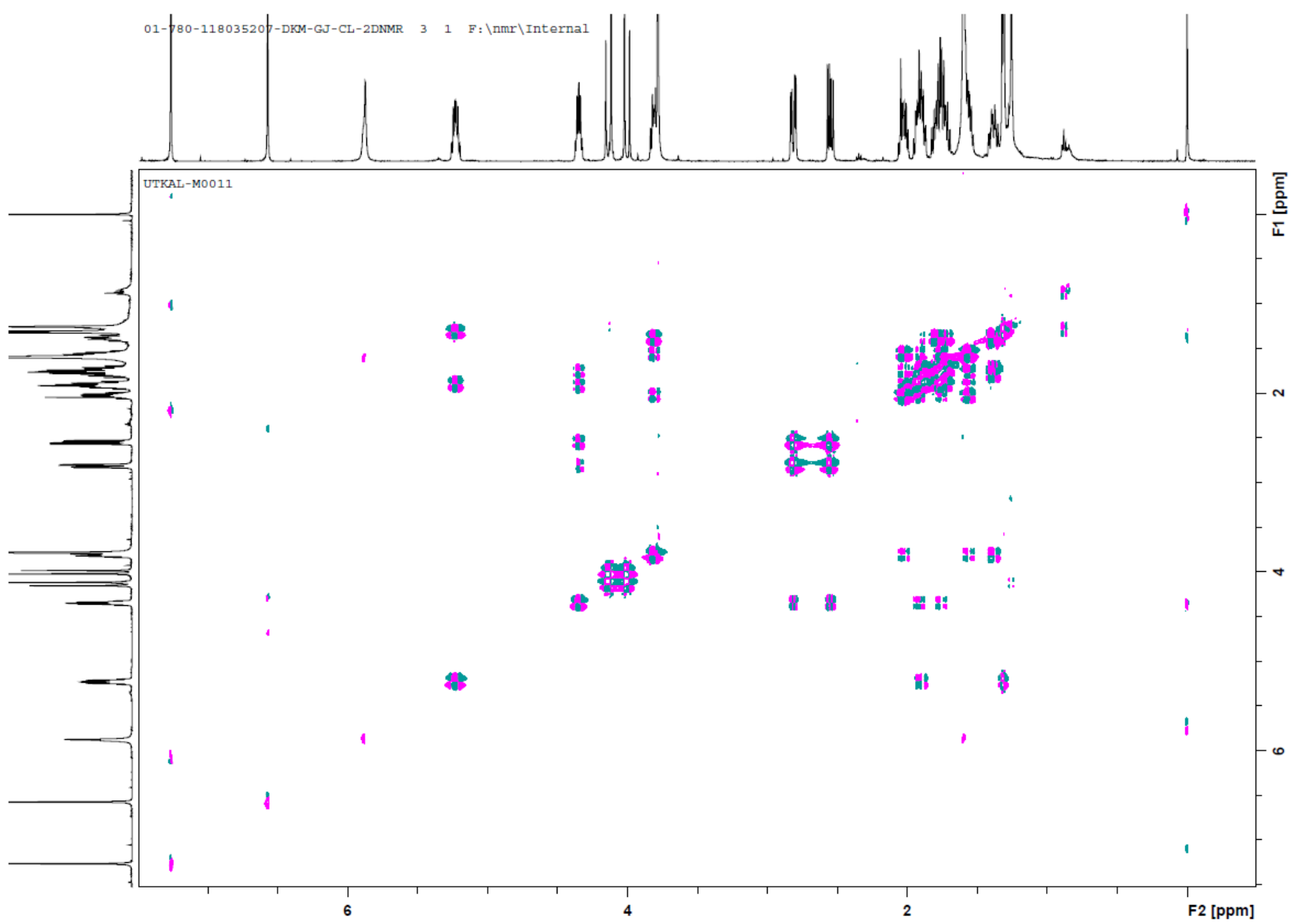

DQF-COSY Spectrum of 5a $\left(500 \mathrm{MHz}, \mathrm{CDCl}_{3}\right)$

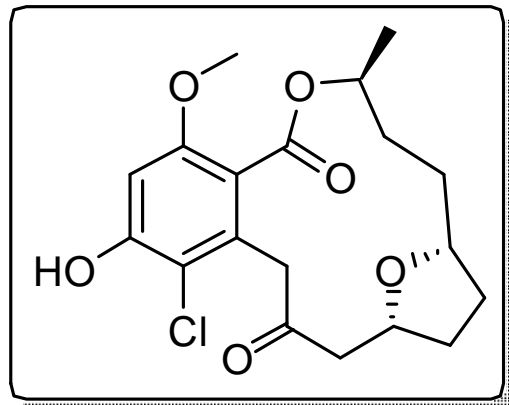




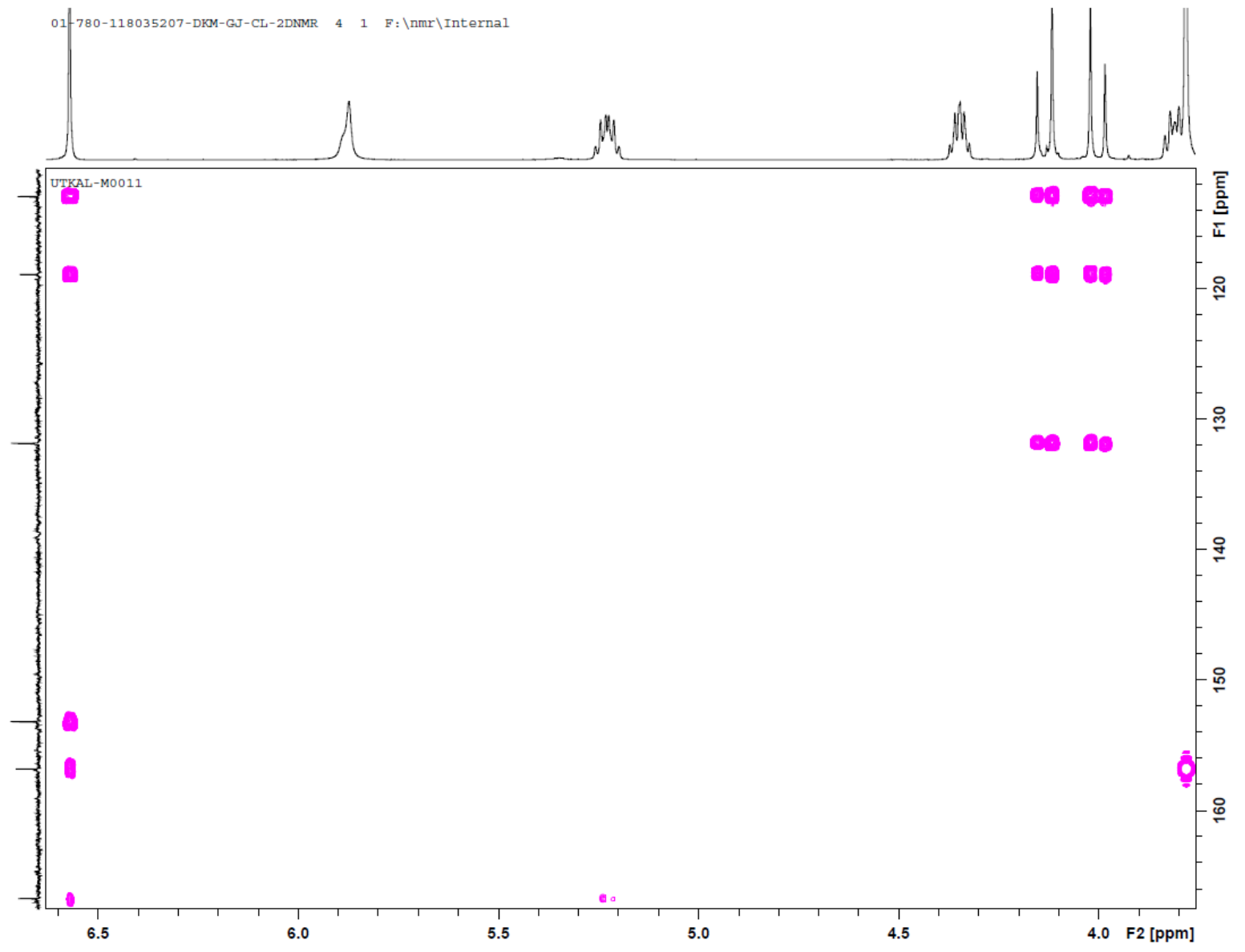

Expanded version of ${ }^{13} \mathrm{C}-{ }^{1} \mathrm{H}$ HMBC spectrum of compound $\mathbf{5 a}\left(500 \mathrm{MHz}, \mathrm{CDCl}_{3}\right)$

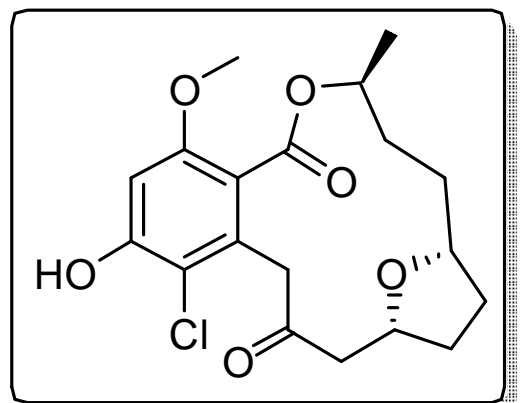




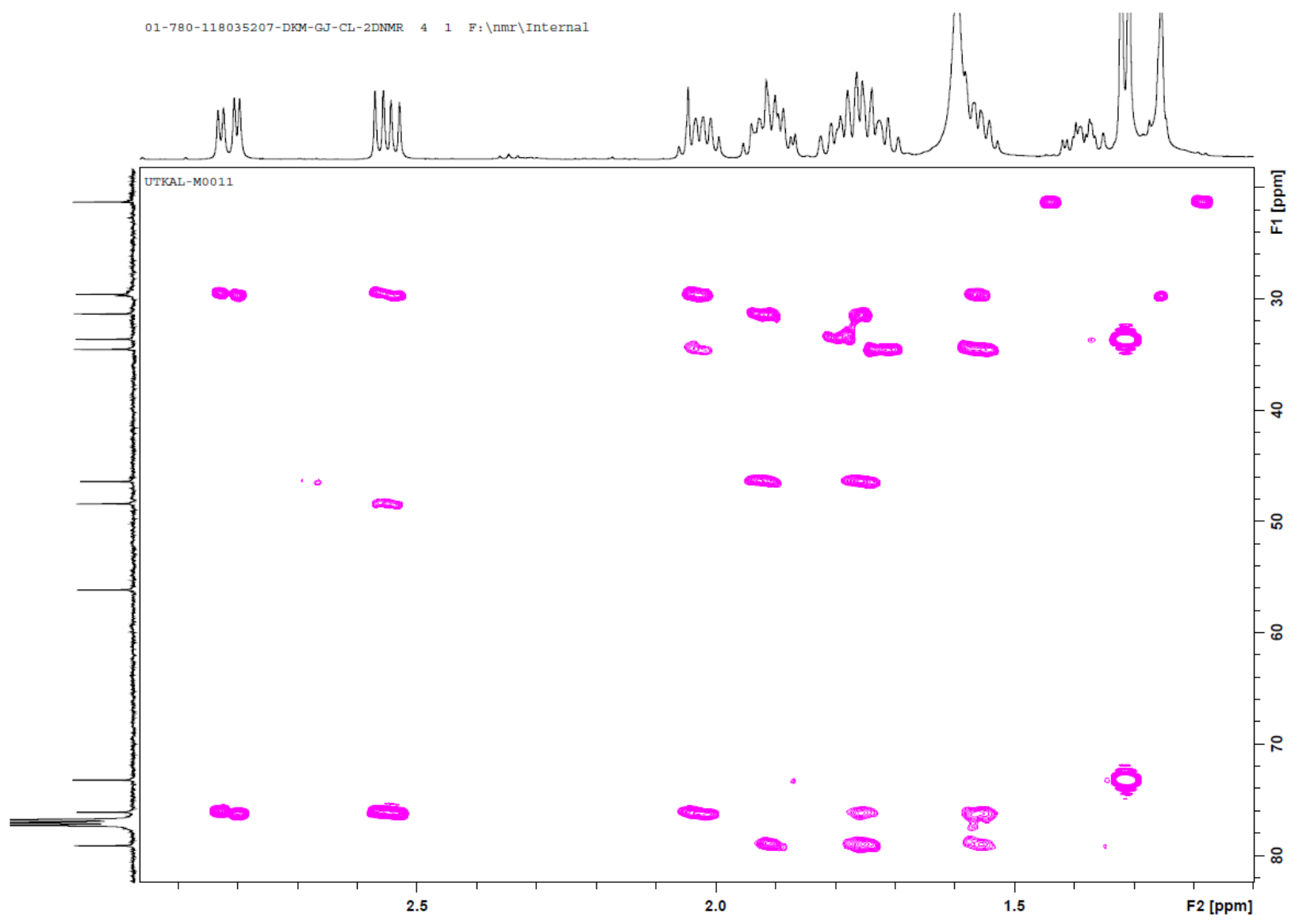

Expanded version of ${ }^{13} \mathrm{C}-{ }^{1} \mathrm{H}$ HMBC spectrum of compound $5 \mathbf{a}\left(500 \mathrm{MHz}, \mathrm{CDCl}_{3}\right)$

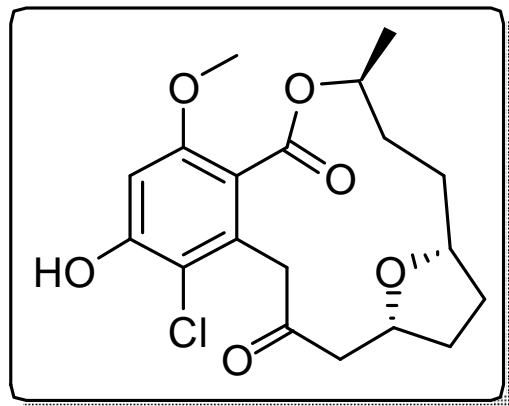




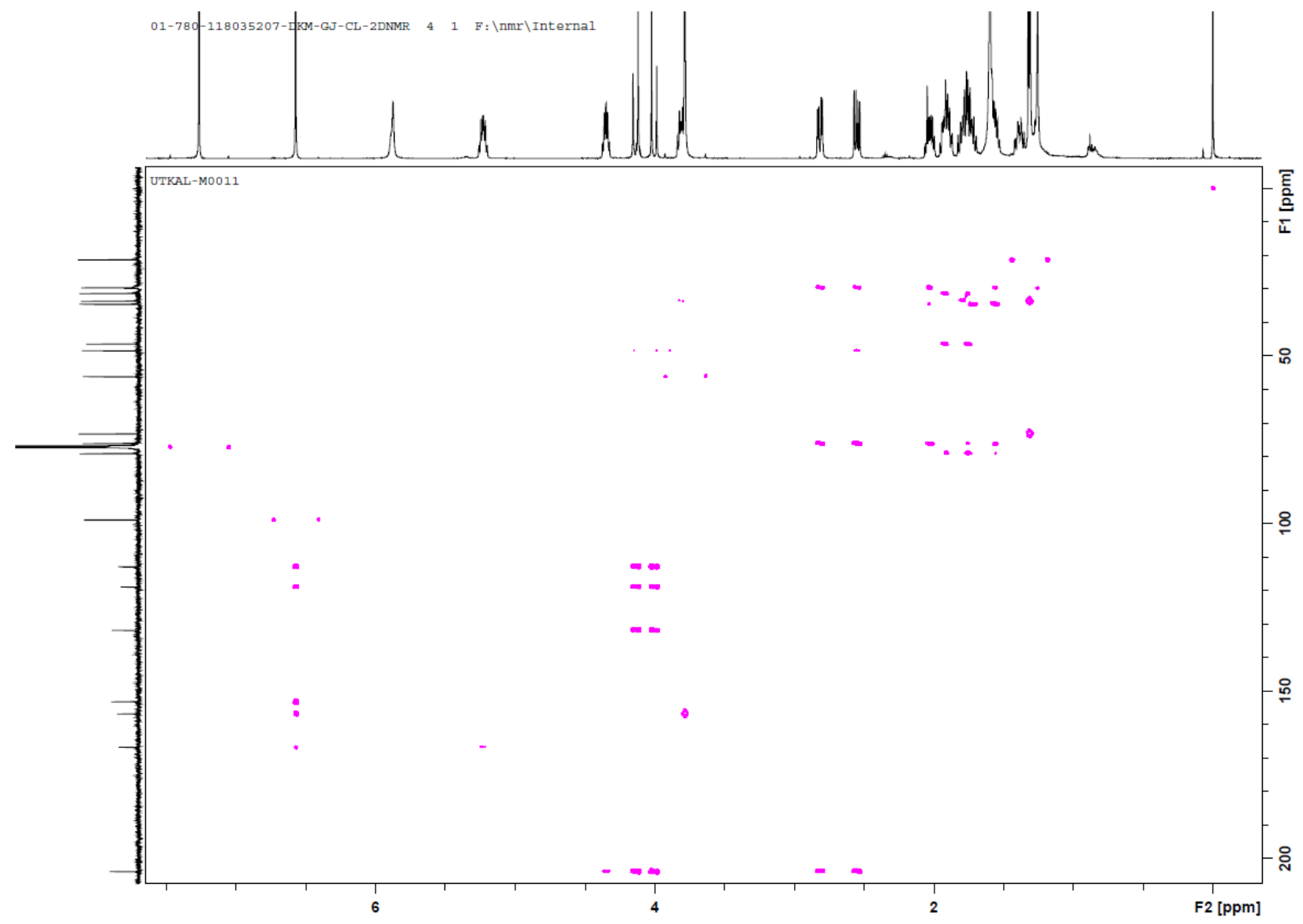

${ }^{13} \mathrm{C}-{ }^{1} \mathrm{H}$ HMBC spectrum of compound $5 \mathbf{a}\left(500 \mathrm{MHz}, \mathrm{CDCl}_{3}\right)$

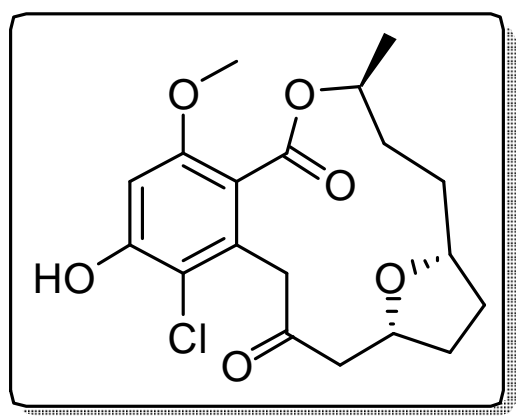




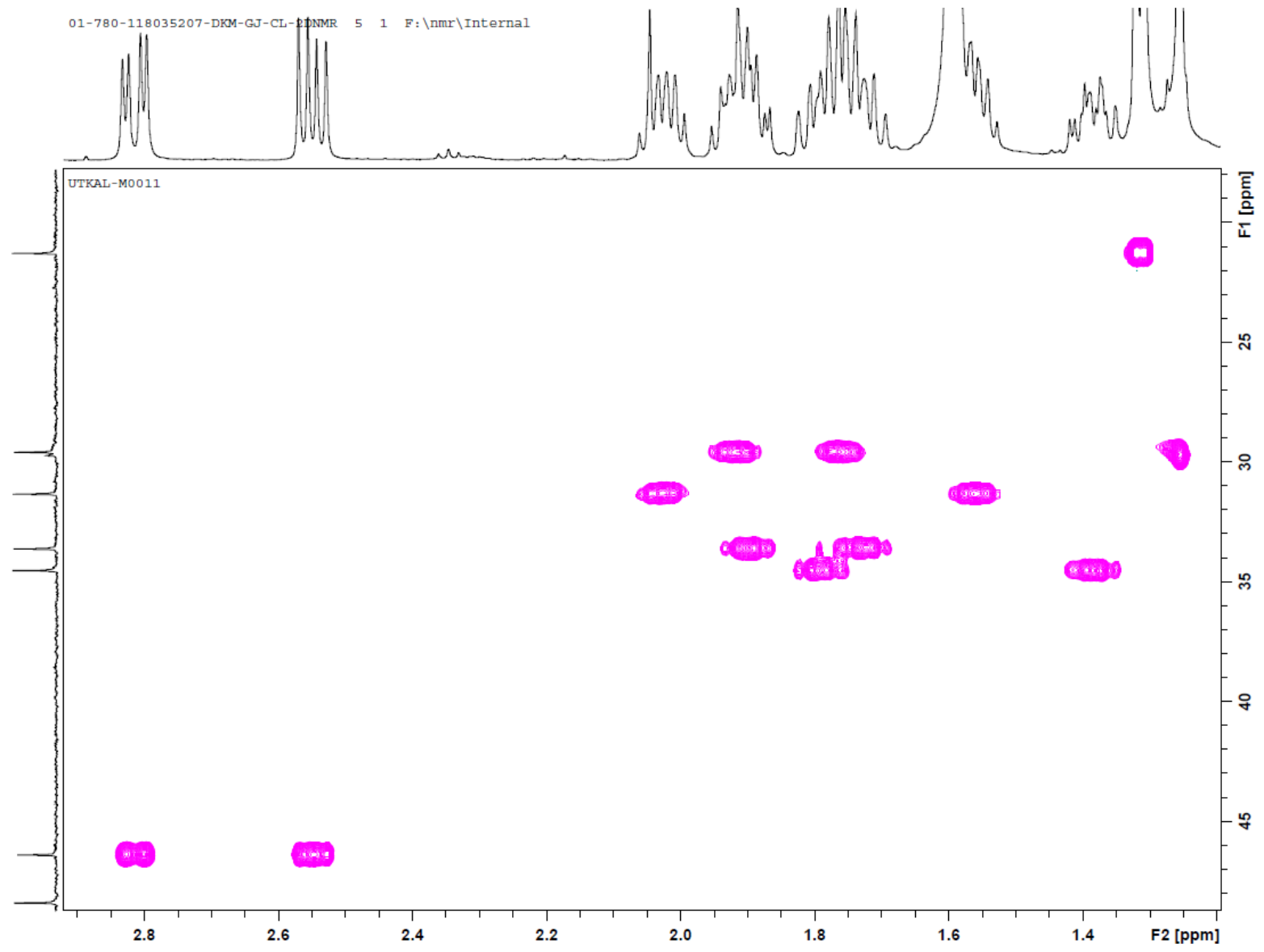

Expanded version of HSQC spectrum of compound $\mathbf{5 a}\left(500 \mathrm{MHz}, \mathrm{CDCl}_{3}\right)$

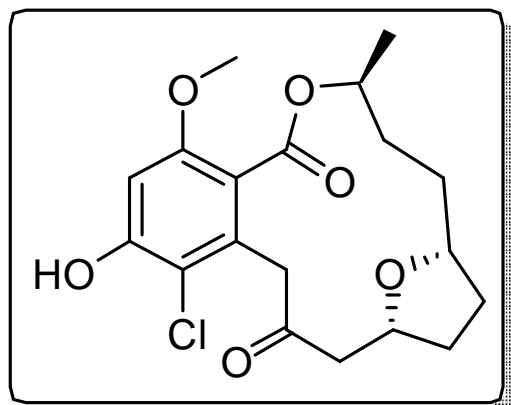




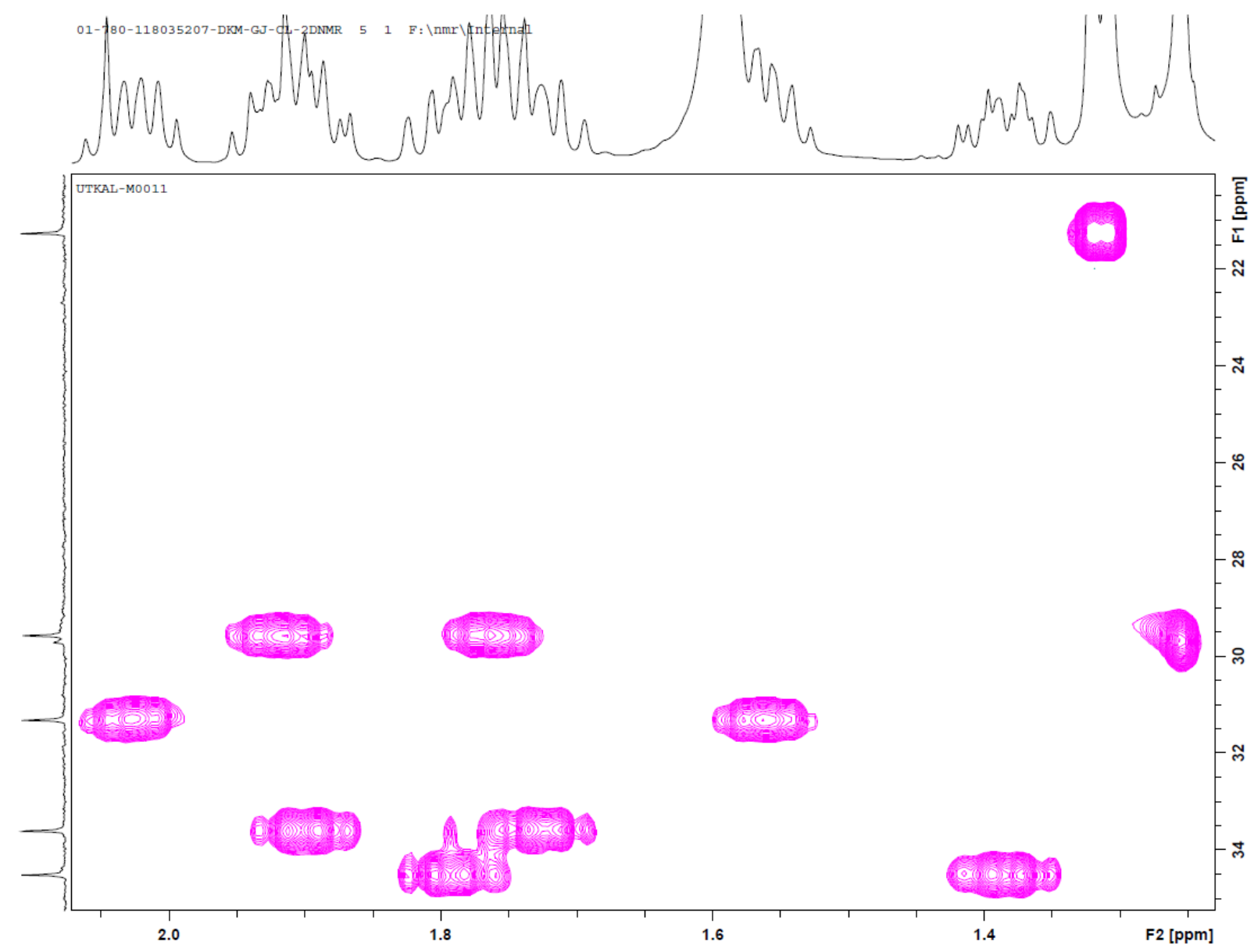

Expanded version of HSQC spectrum of compound $5 \mathbf{a}\left(500 \mathrm{MHz}, \mathrm{CDCl}_{3}\right)$

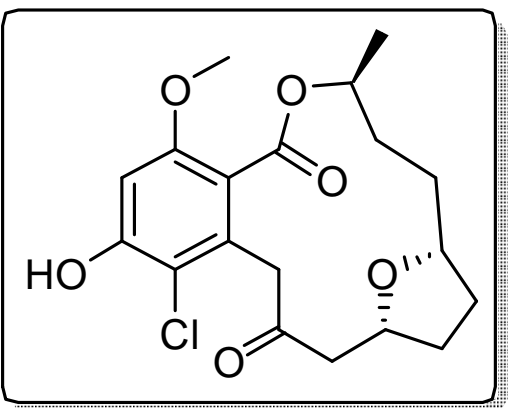




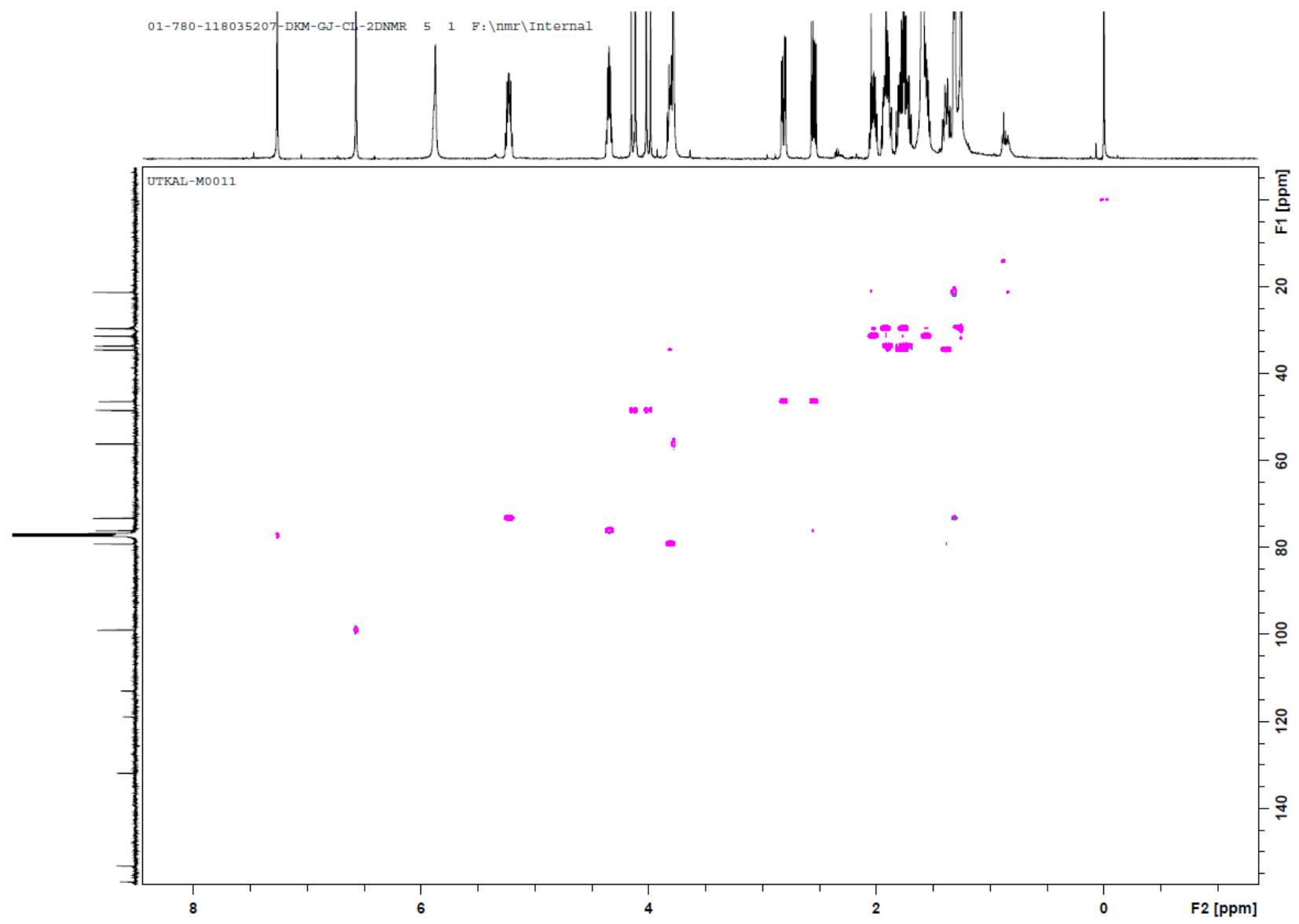

HSQC spectrum of compound $\mathbf{5 a}\left(500 \mathrm{MHz}, \mathrm{CDCl}_{3}\right)$

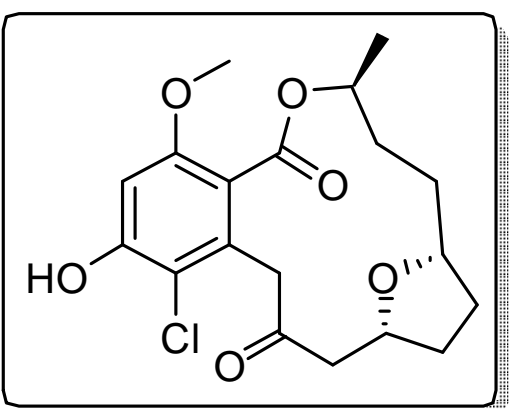




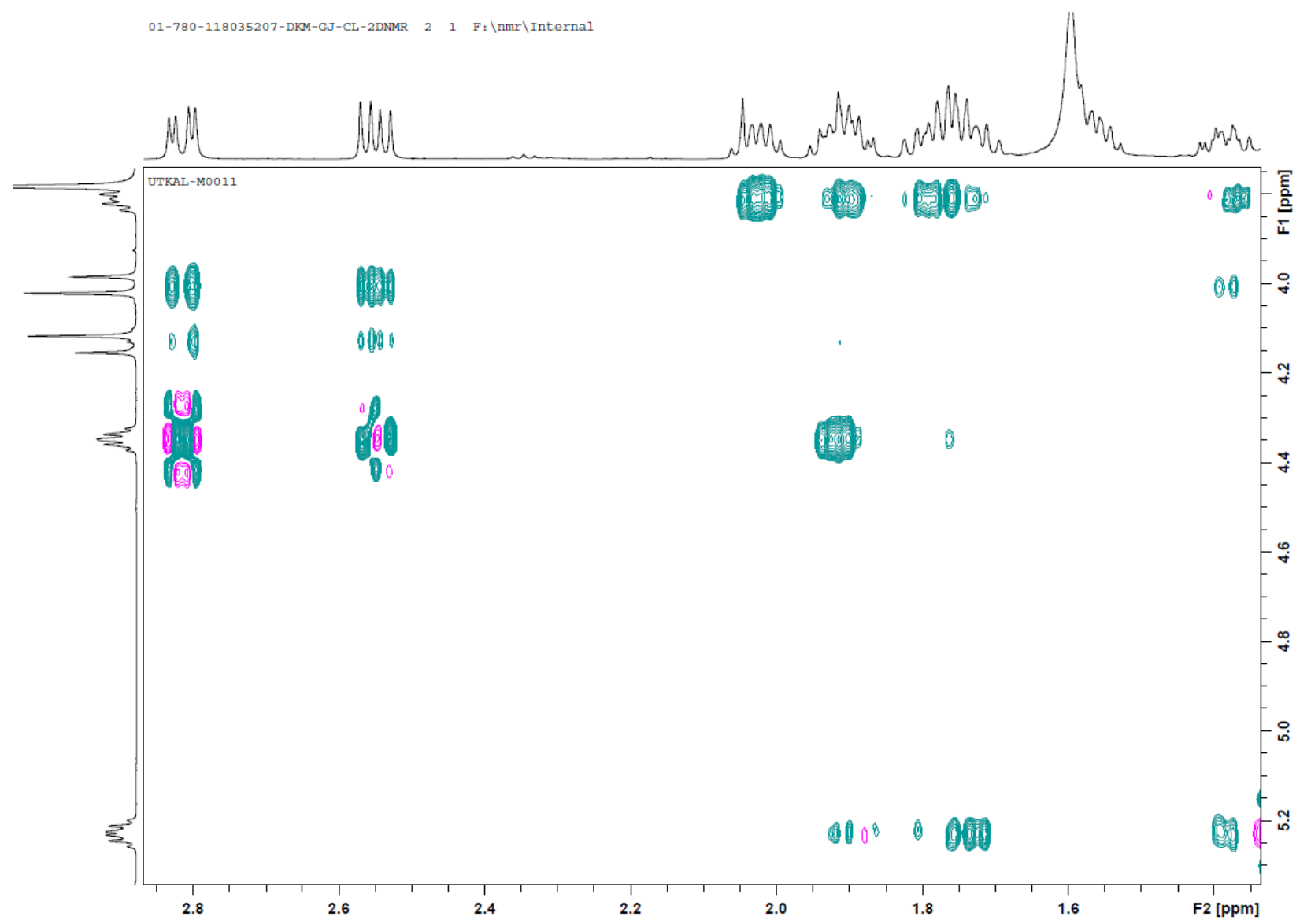

Expanded version of NOESY spectrum of compound $5 \mathbf{a}\left(500 \mathrm{MHz}, \mathrm{CDCl}_{3}\right)$

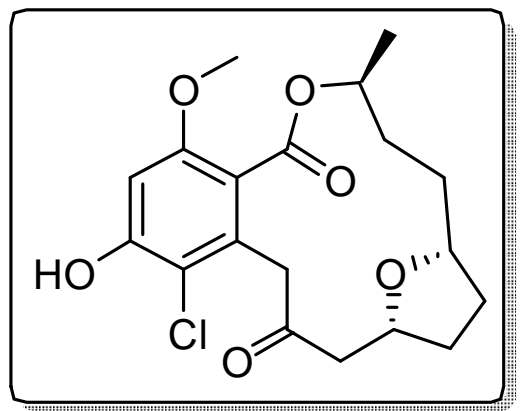




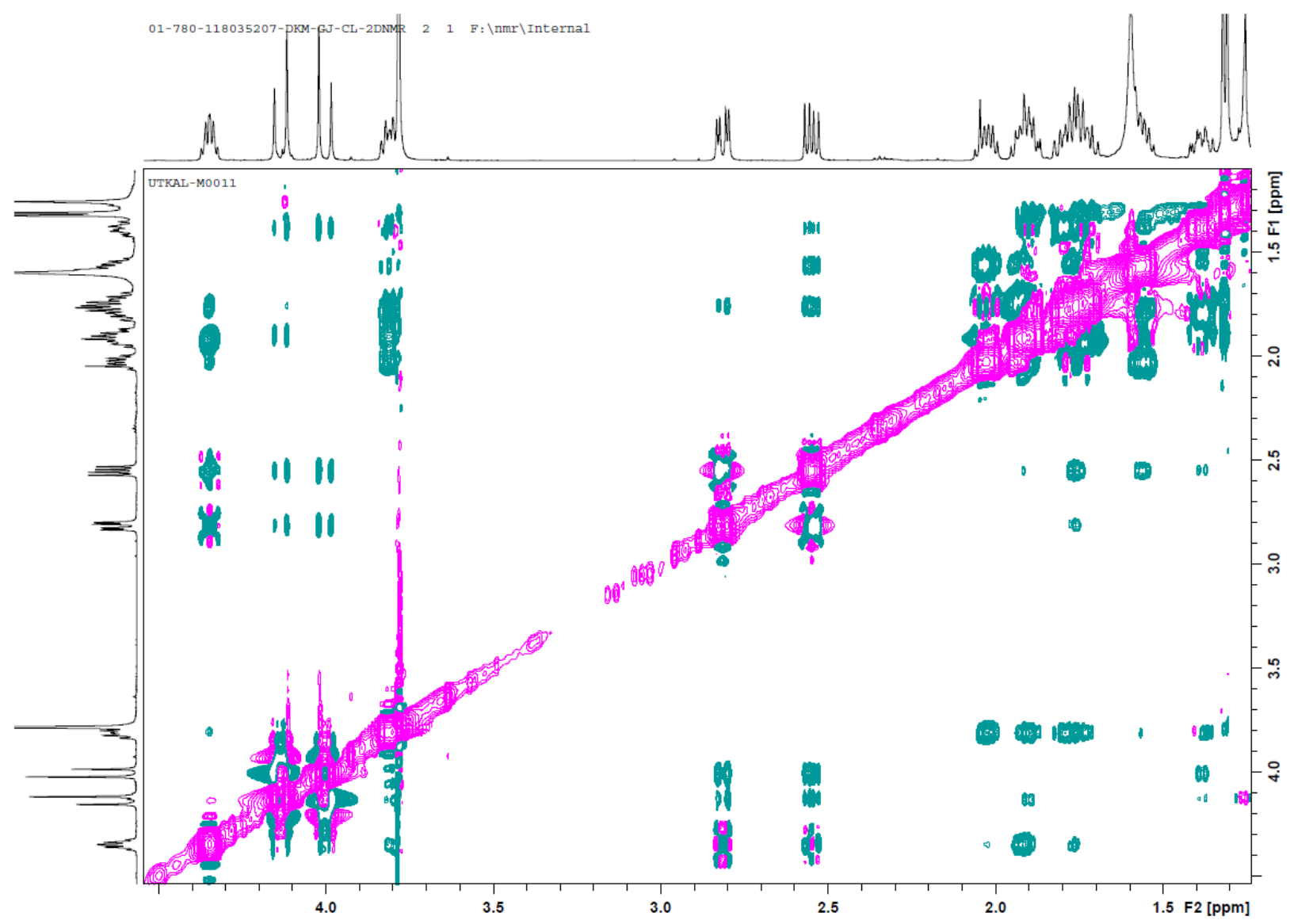

Expanded version of NOESY spectrum of compound $5 \mathbf{a}\left(500 \mathrm{MHz}, \mathrm{CDCl}_{3}\right)$

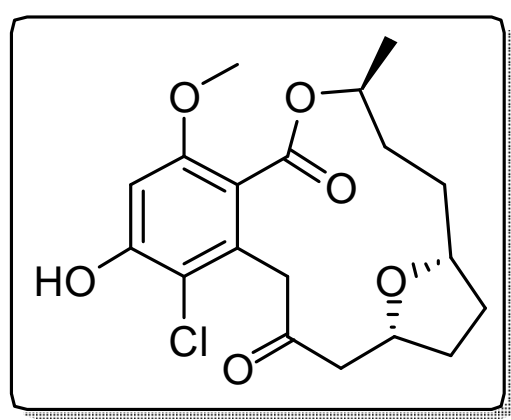




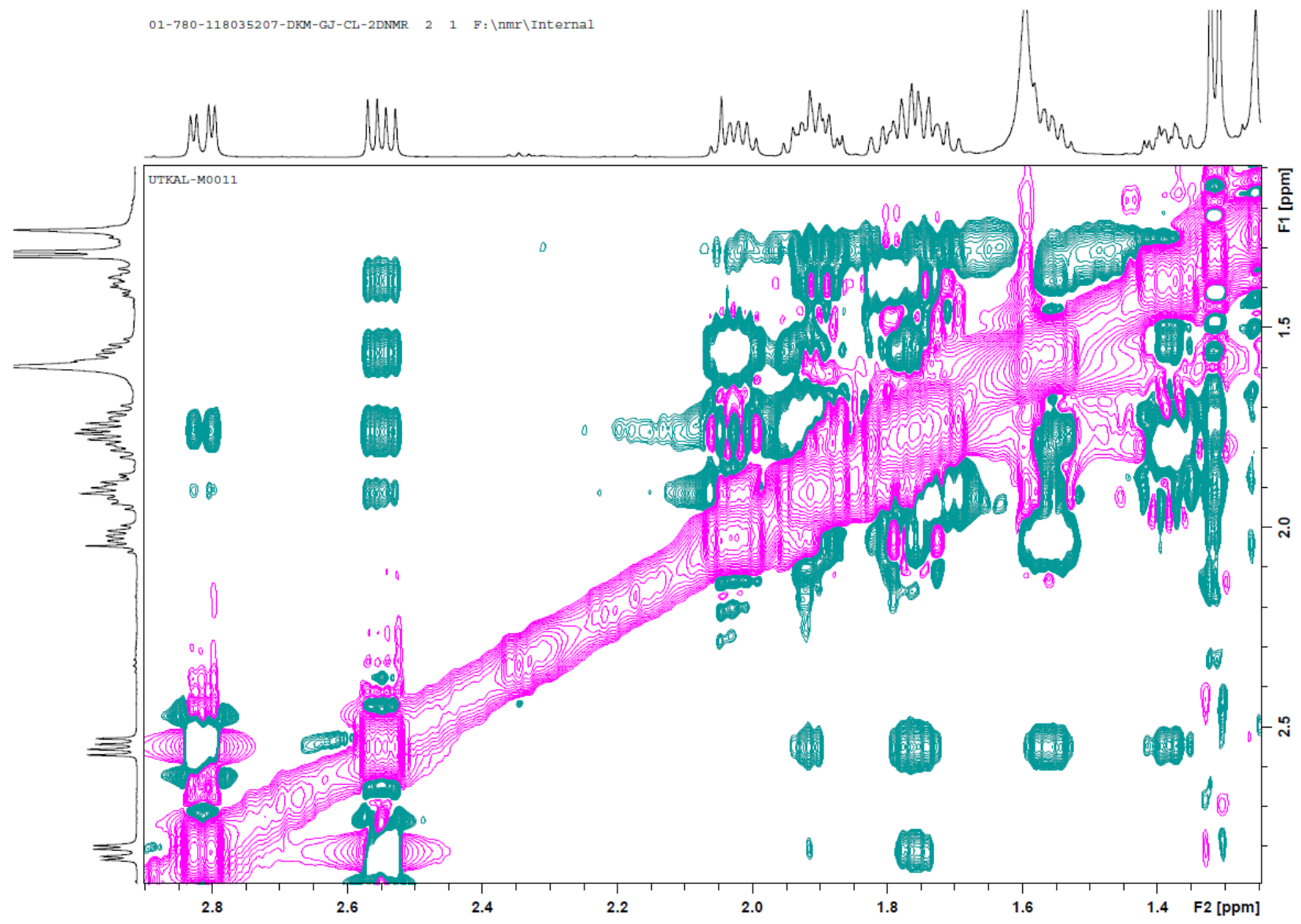

Expanded version of NOESY spectrum of compound $\mathbf{5 a}\left(500 \mathrm{MHz}, \mathrm{CDCl}_{3}\right)$

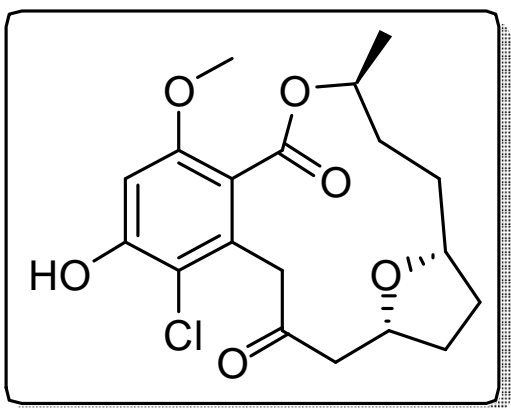




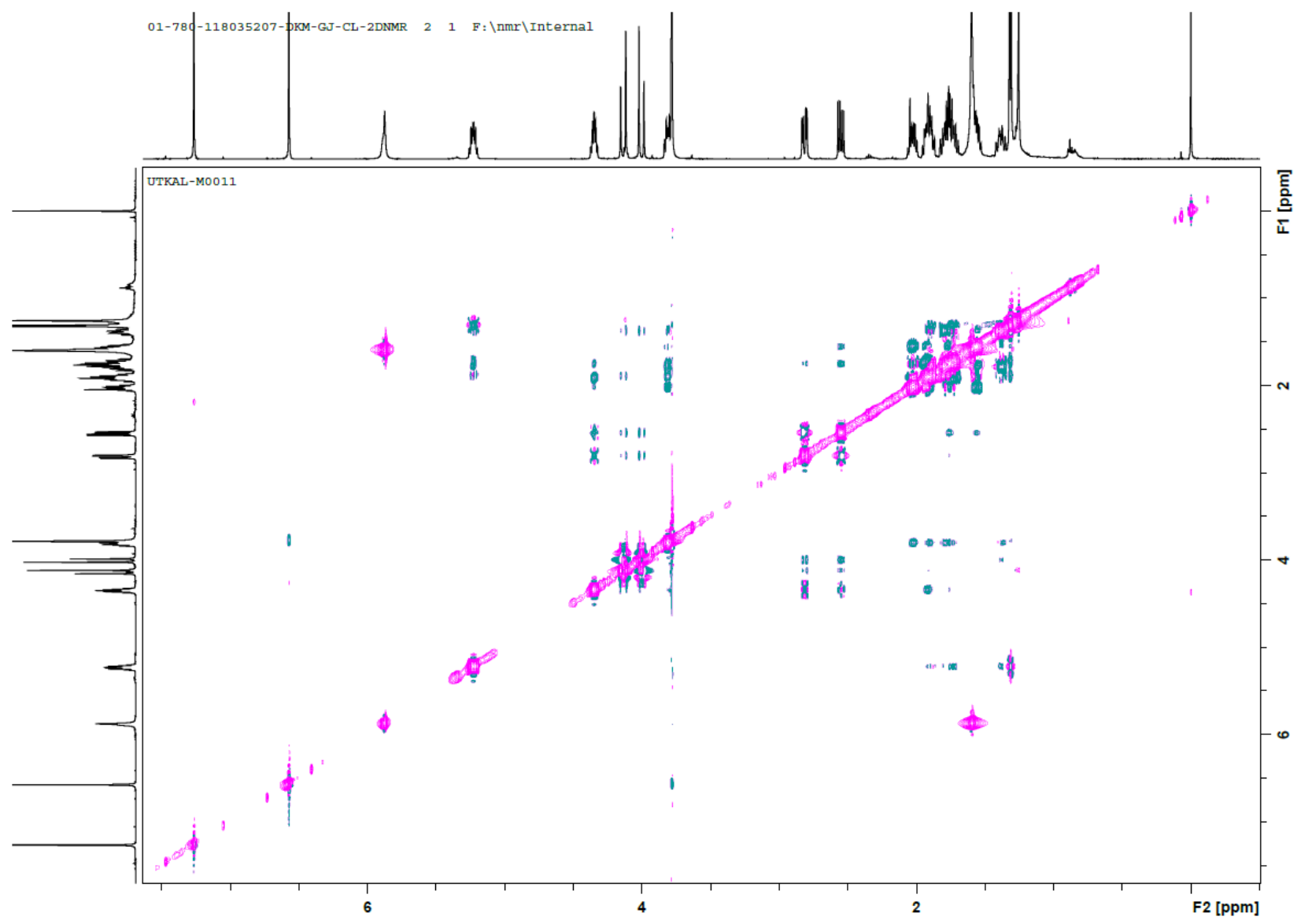

NOESY spectrum of compound $\mathbf{5 a}\left(500 \mathrm{MHz}, \mathrm{CDCl}_{3}\right)$

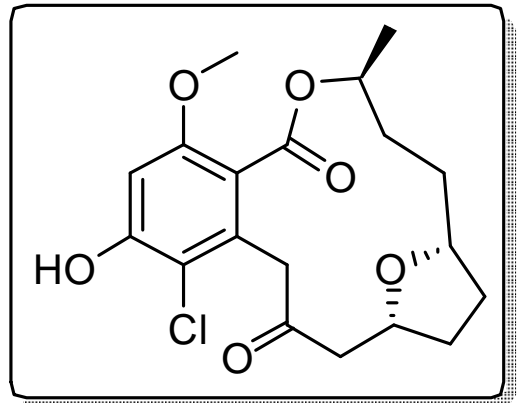




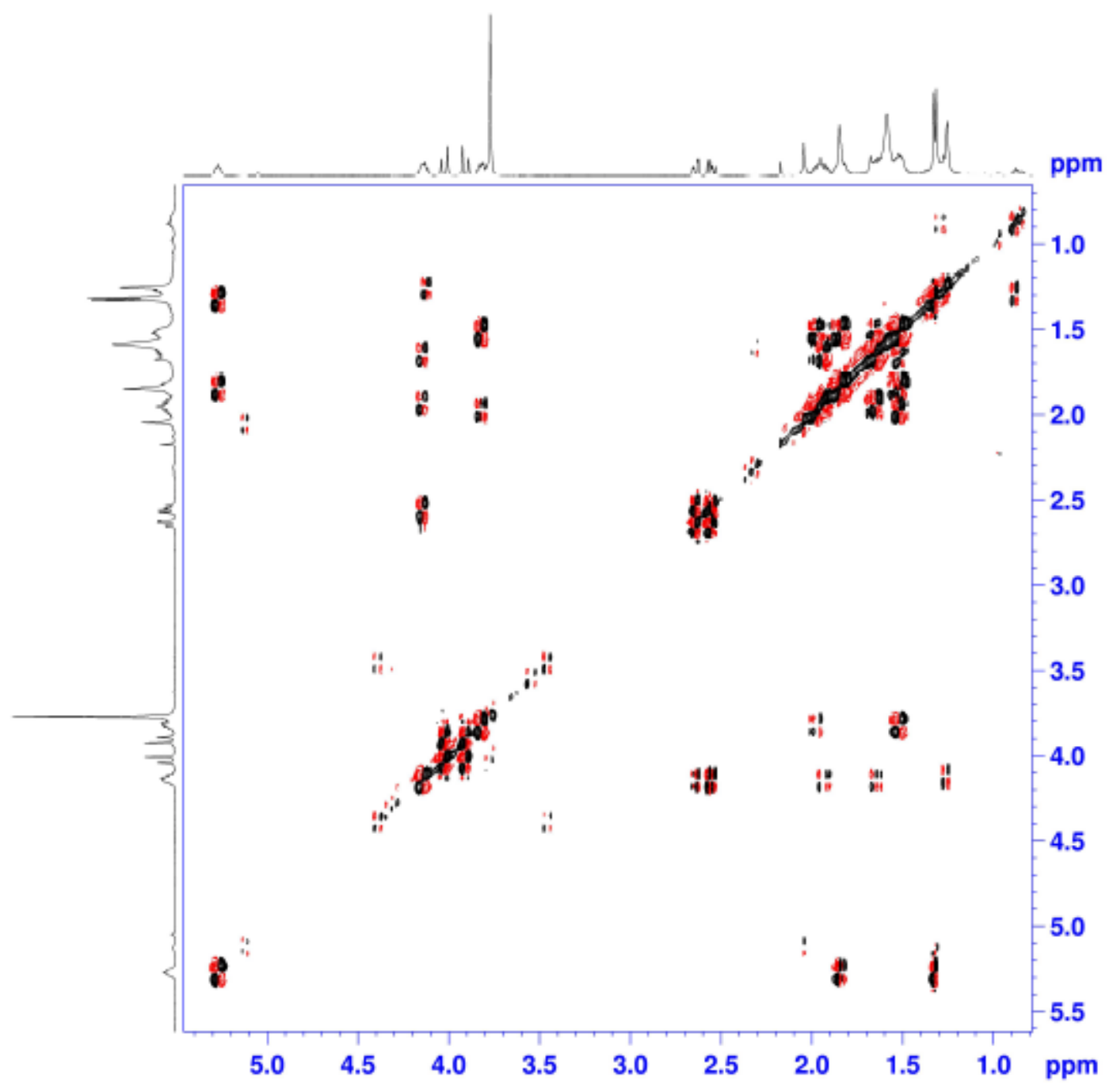

Expanded version of DQF-COSY spectrum of compound $4 \mathbf{a}\left(500 \mathrm{MHz}, \mathrm{CDCl}_{3}\right)$

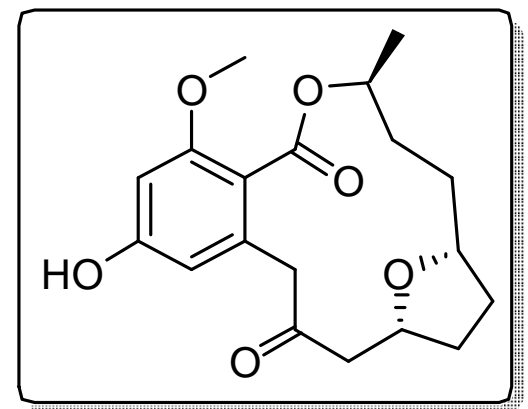




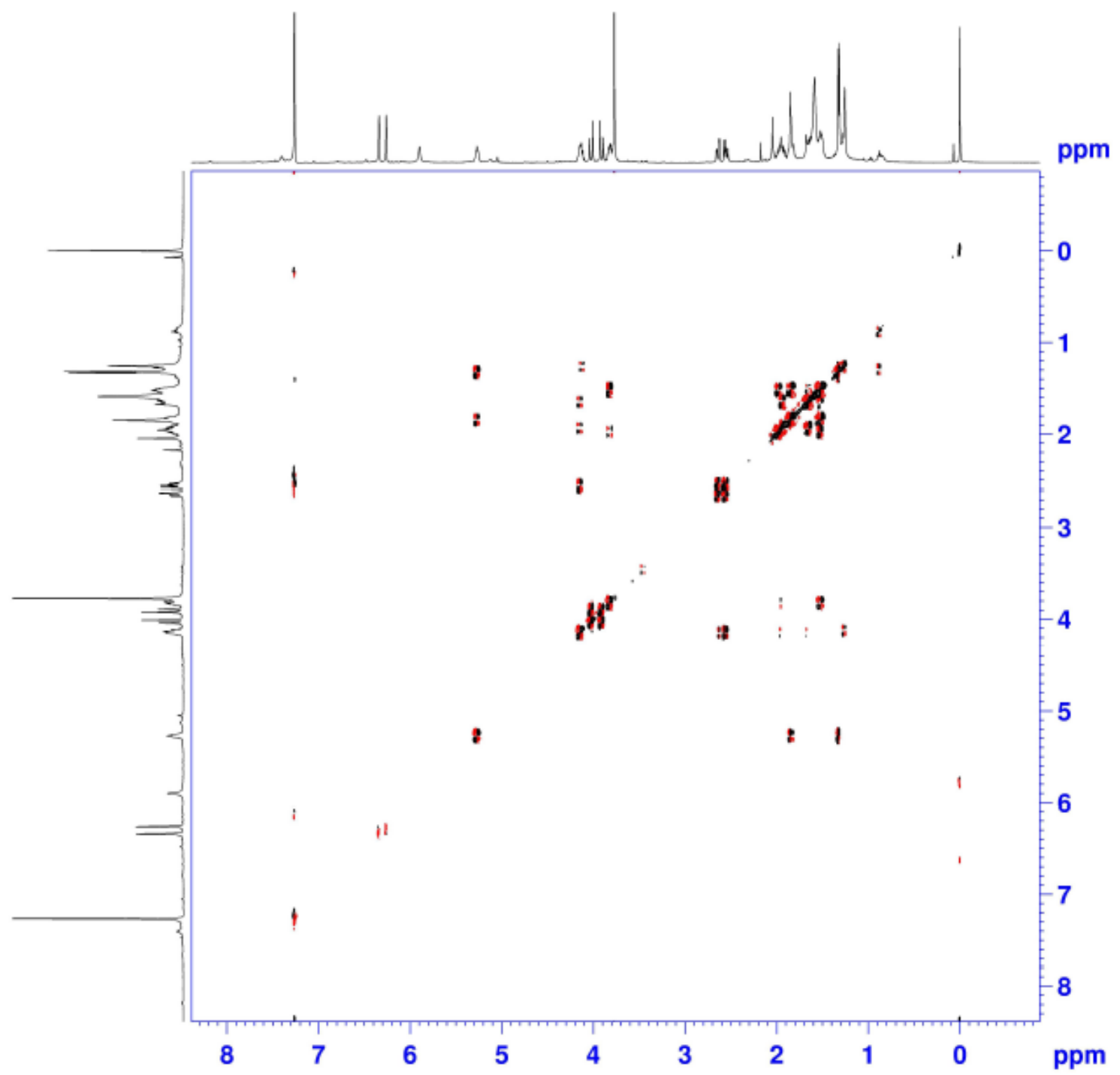

DQF-COSY spectrum of compound $4 \mathbf{a}\left(500 \mathrm{MHz}, \mathrm{CDCl}_{3}\right)$

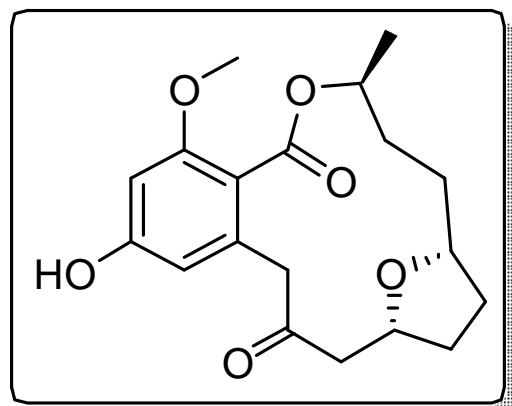




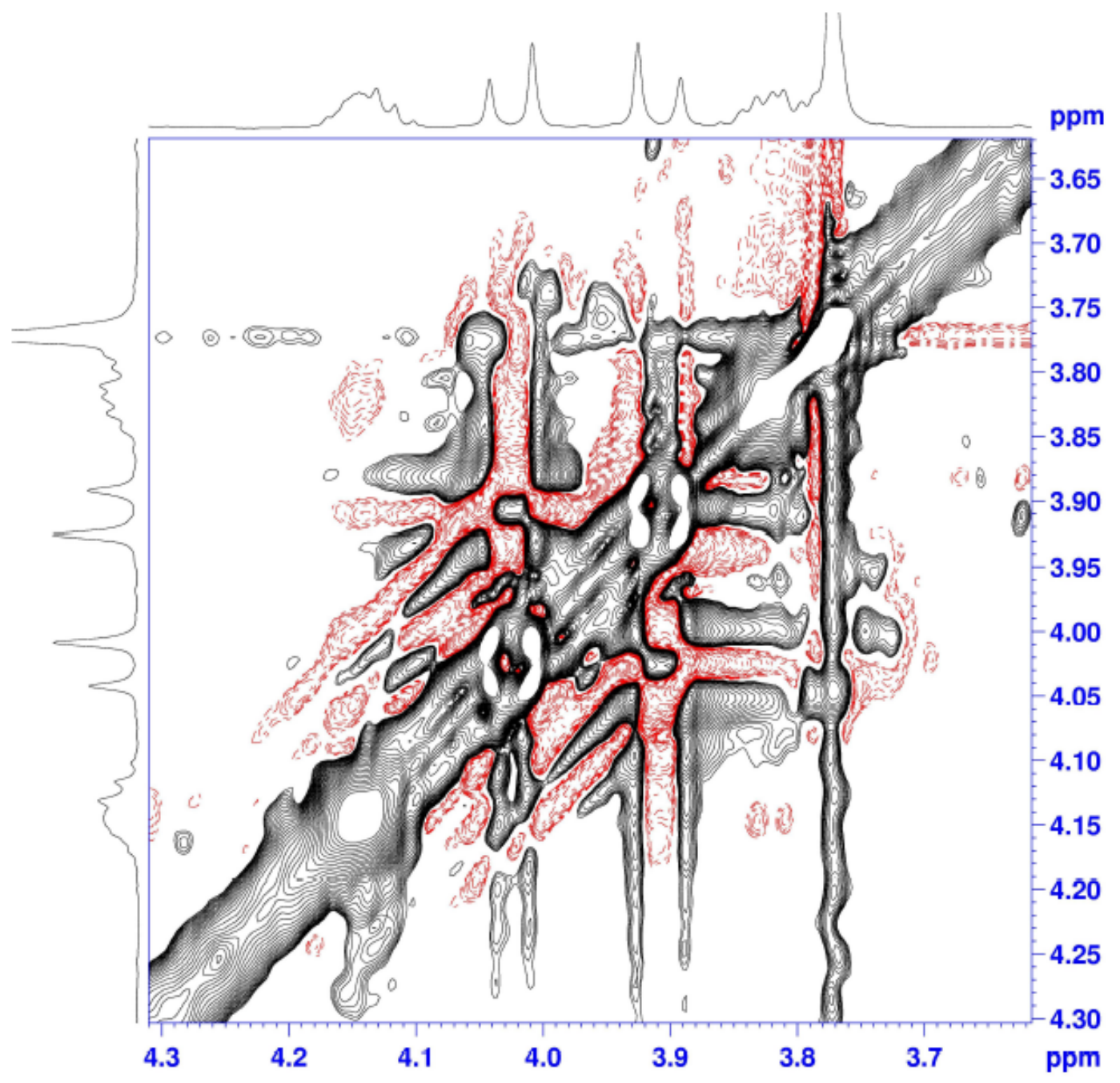

Expanded version of NOESY spectrum of compound $4 \mathbf{a}\left(500 \mathrm{MHz}, \mathrm{CDCl}_{3}\right)$

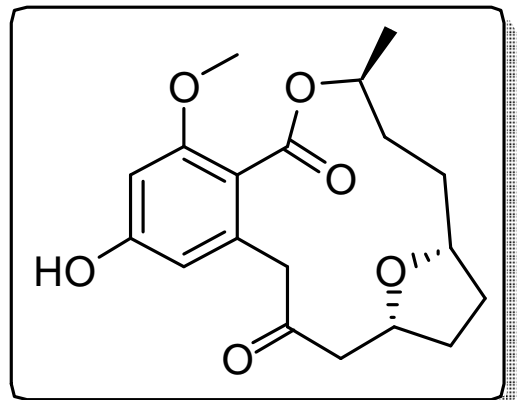




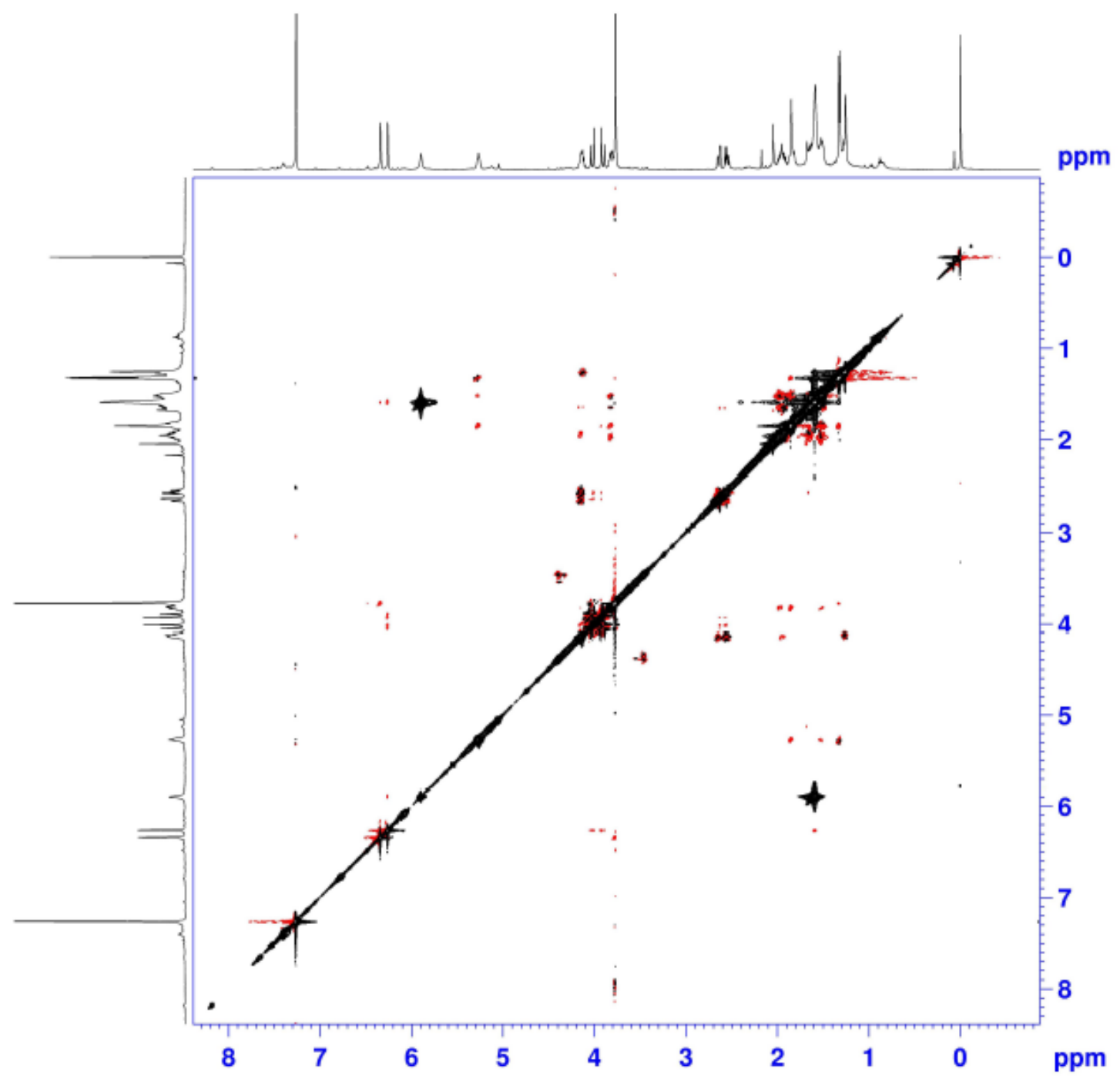

NOESY spectrum of compound $\mathbf{4 a}\left(500 \mathrm{MHz}, \mathrm{CDCl}_{3}\right)$

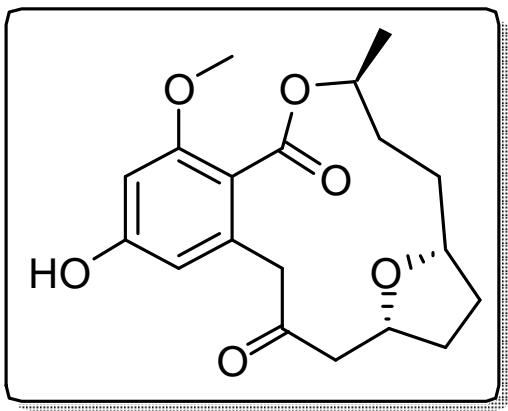




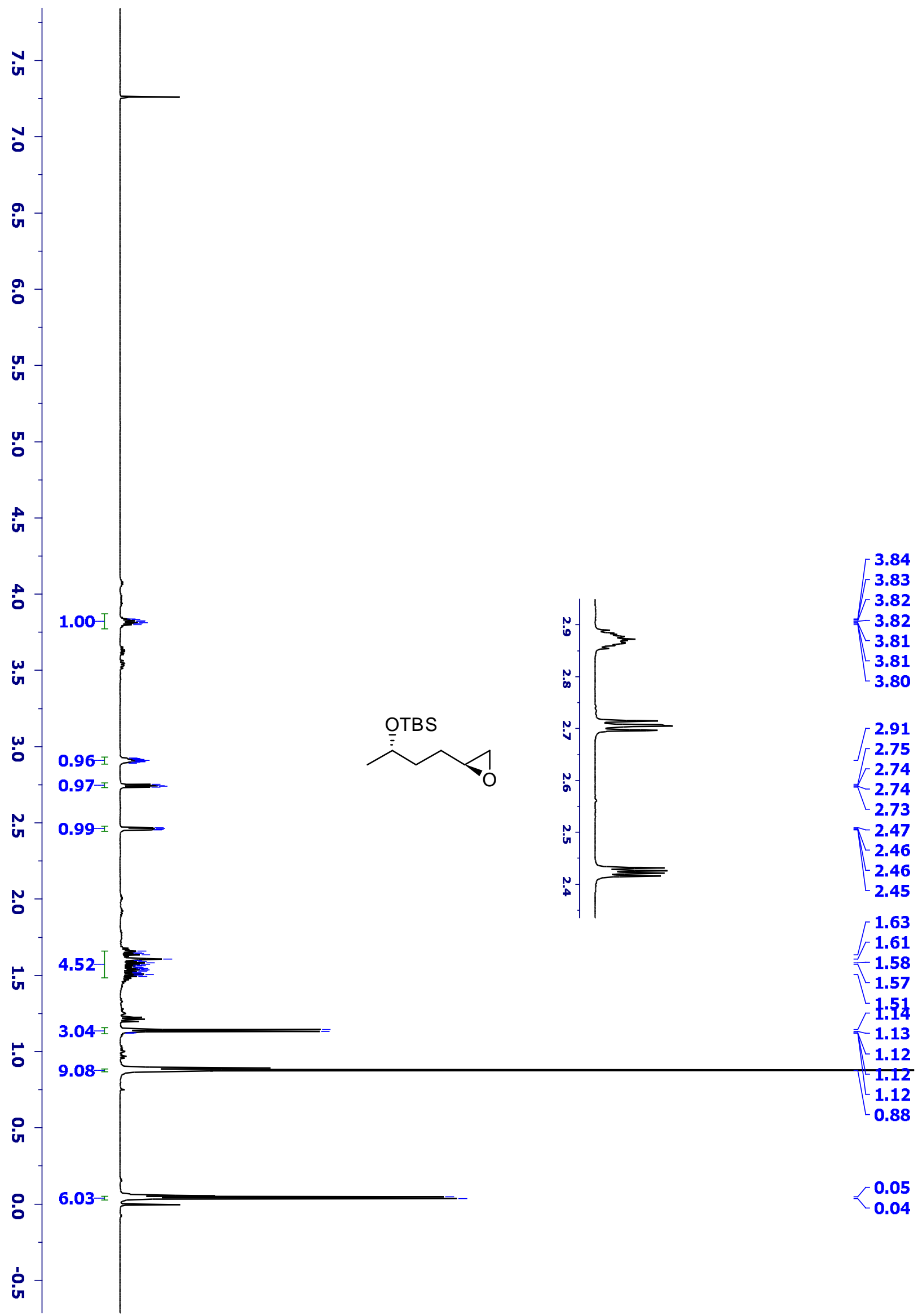

${ }^{1} \mathrm{H}$ NMR spectrum of $\mathbf{1 7 a}\left(500 \mathrm{MHz}, \mathrm{CDCl}_{3}\right)$ 


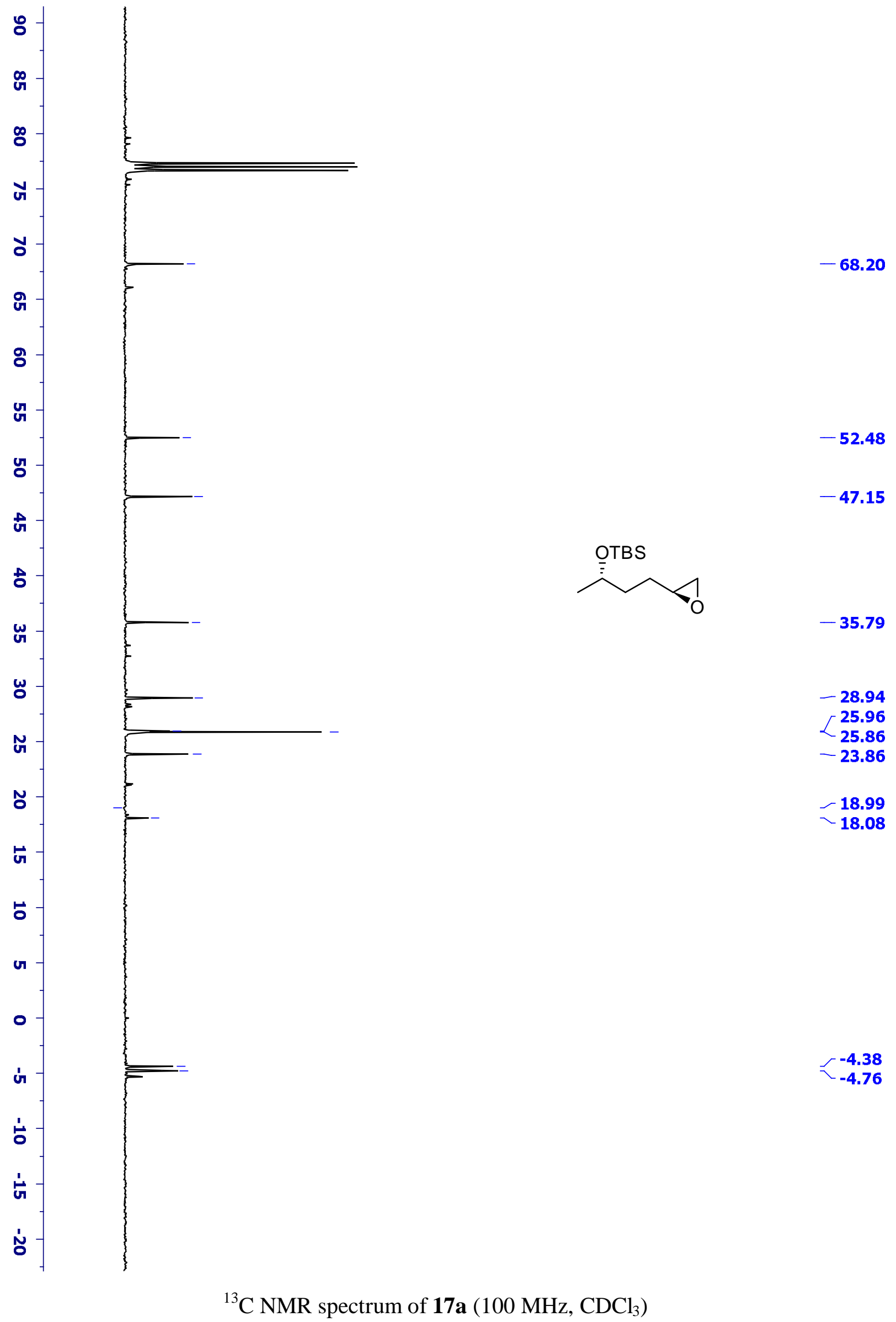




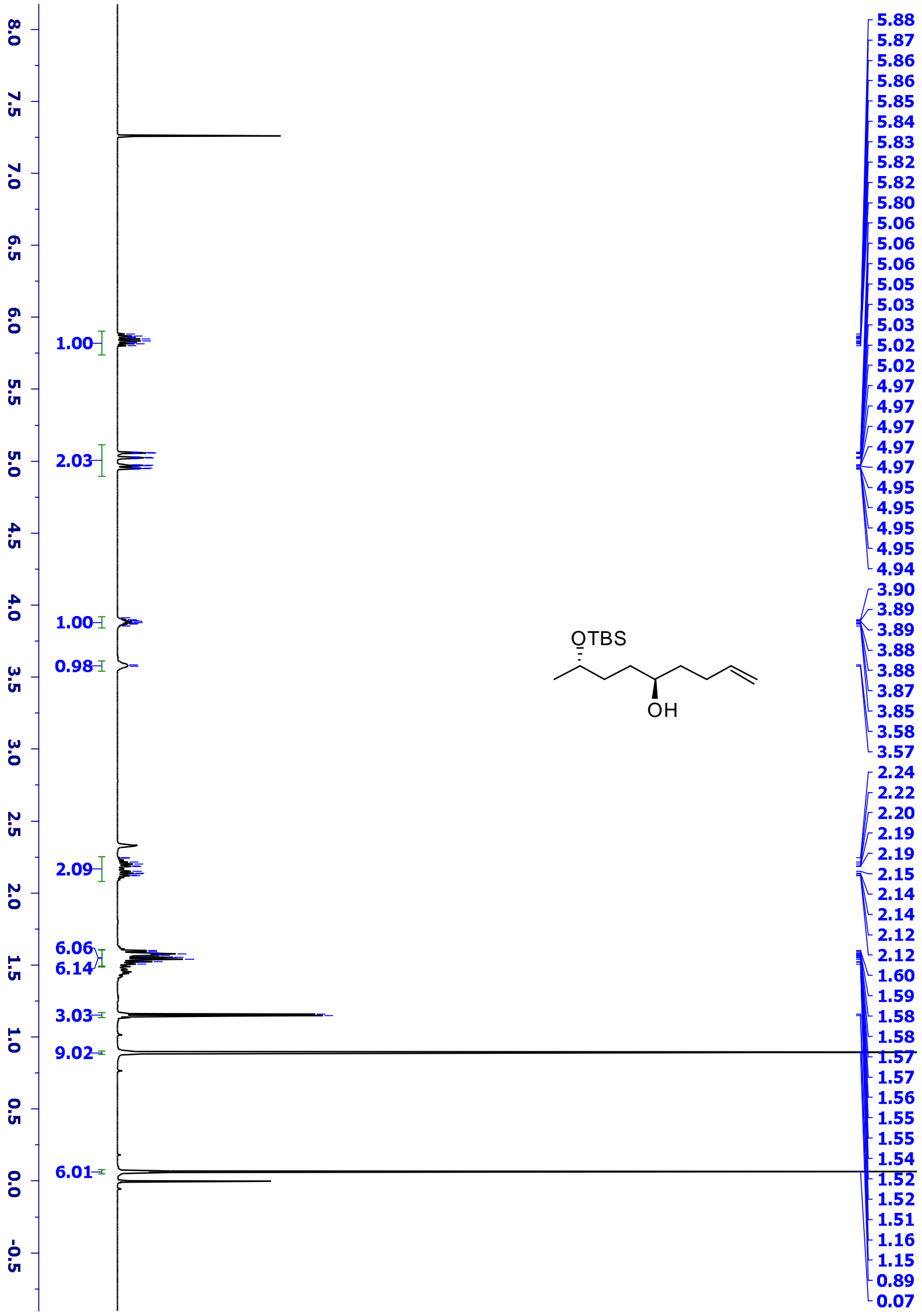

${ }^{1} \mathrm{H}$ NMR spectrum of $\mathbf{1 8 a}\left(500 \mathrm{MHz}, \mathrm{CDCl}_{3}\right)$ 


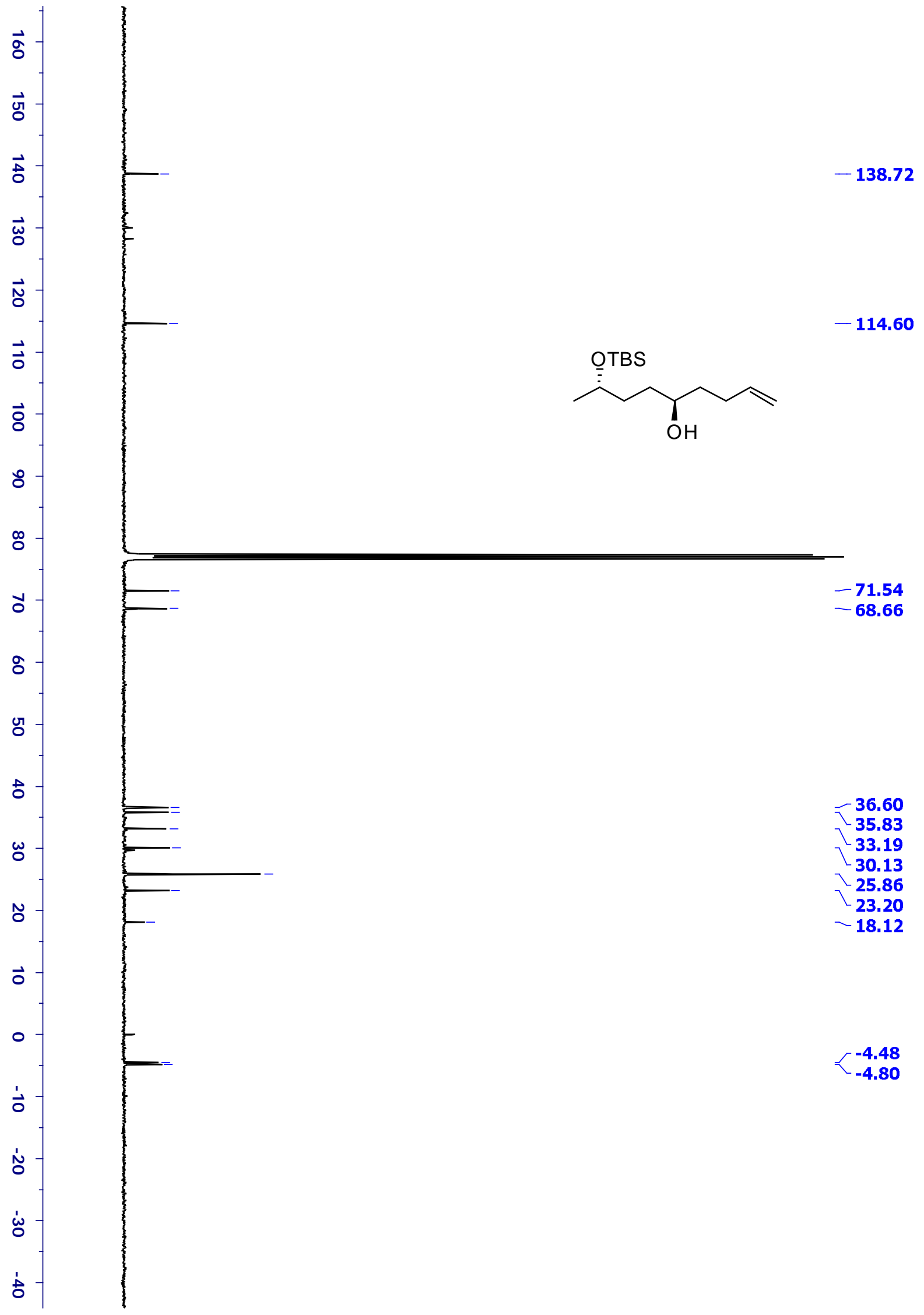

${ }^{13} \mathrm{C}$ NMR spectrum of $\mathbf{1 8 a}\left(100 \mathrm{MHz}, \mathrm{CDCl}_{3}\right)$ 


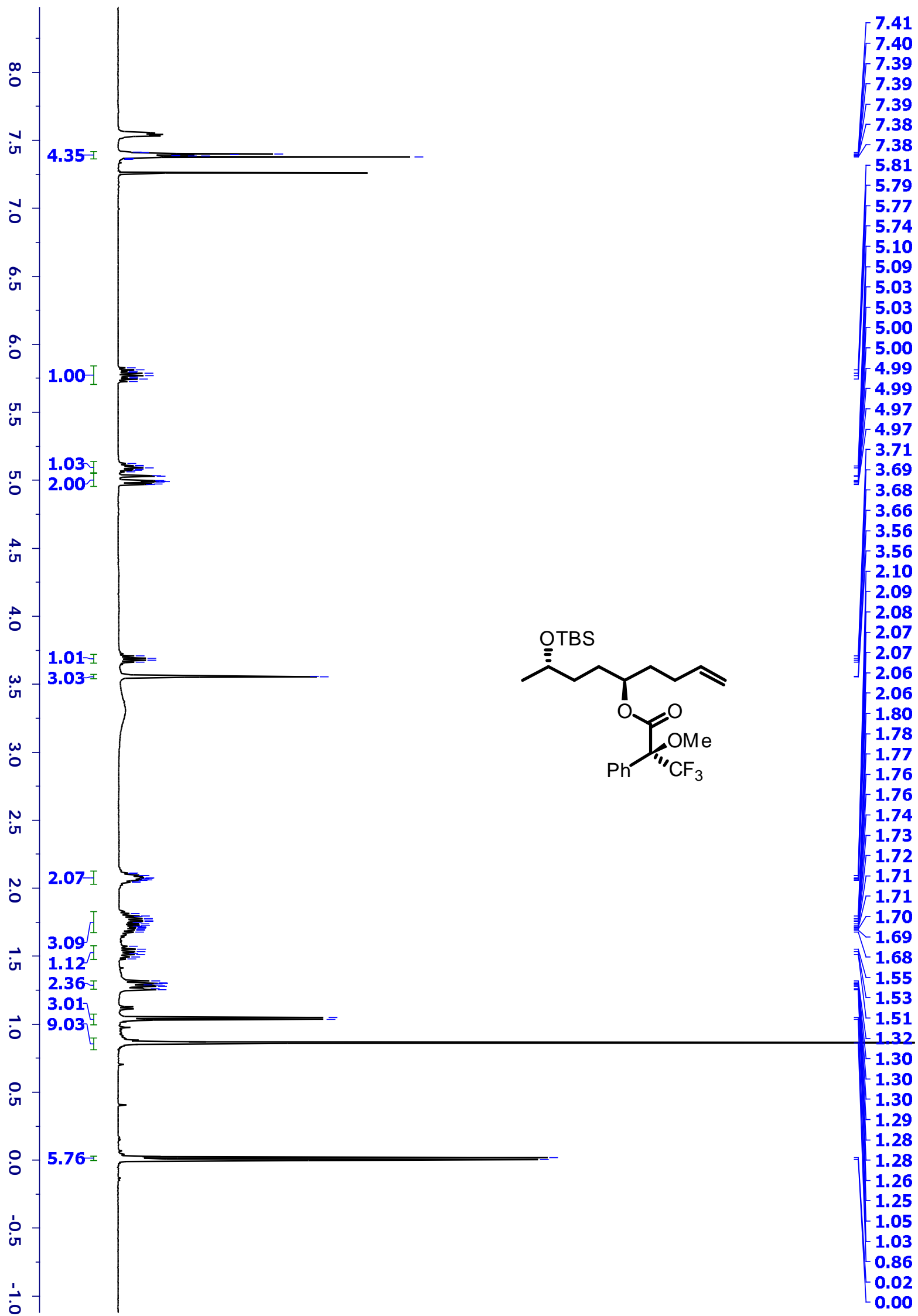

${ }^{1} \mathrm{H}$ NMR spectrum of $\mathbf{1 8 a} \mathbf{a}^{\prime}\left(500 \mathrm{MHz}, \mathrm{CDCl}_{3}\right)$ 


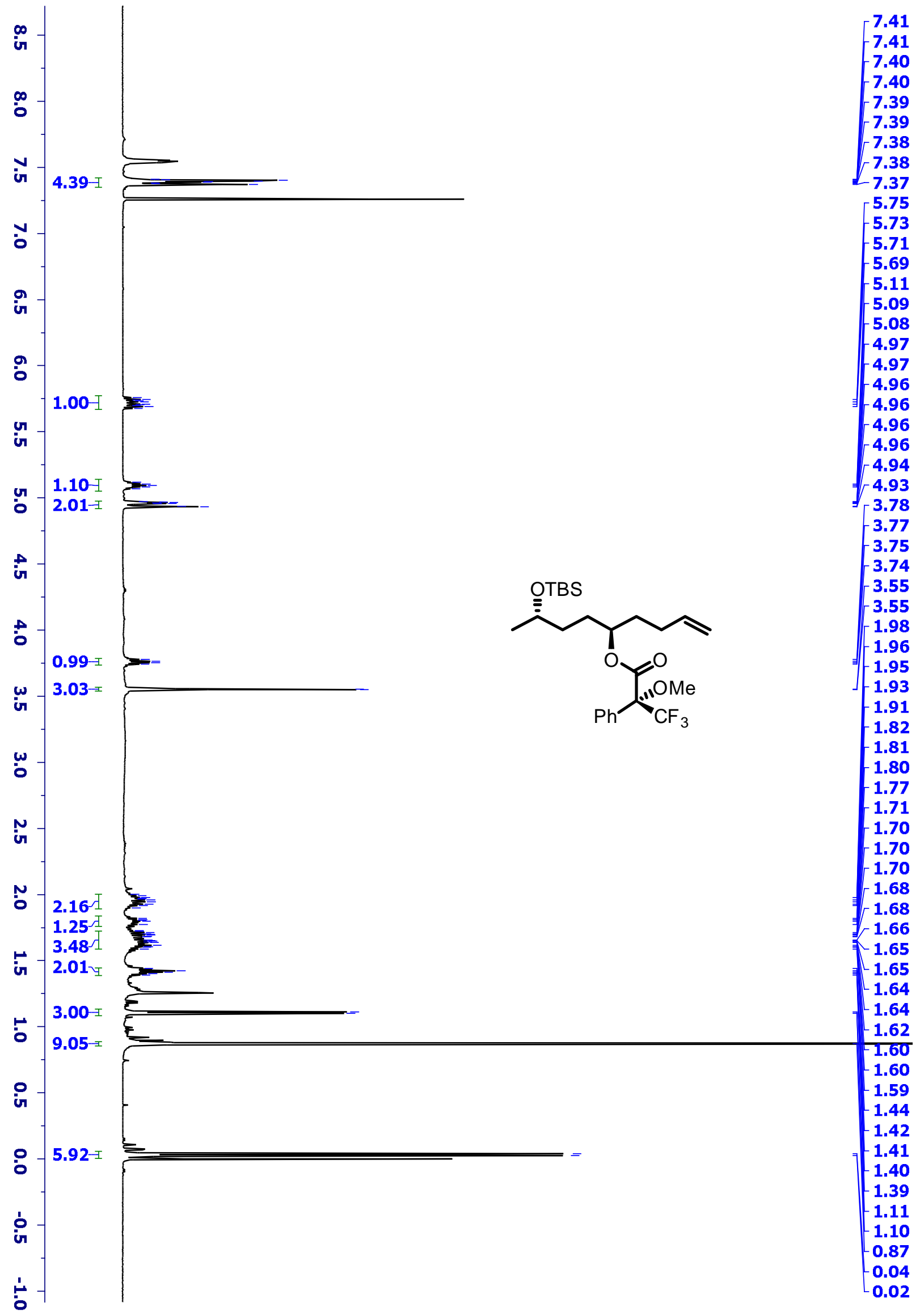

${ }^{1} \mathrm{H}$ NMR spectrum of $18 a^{\prime \prime}\left(500 \mathrm{MHz}, \mathrm{CDCl}_{3}\right.$ ) 


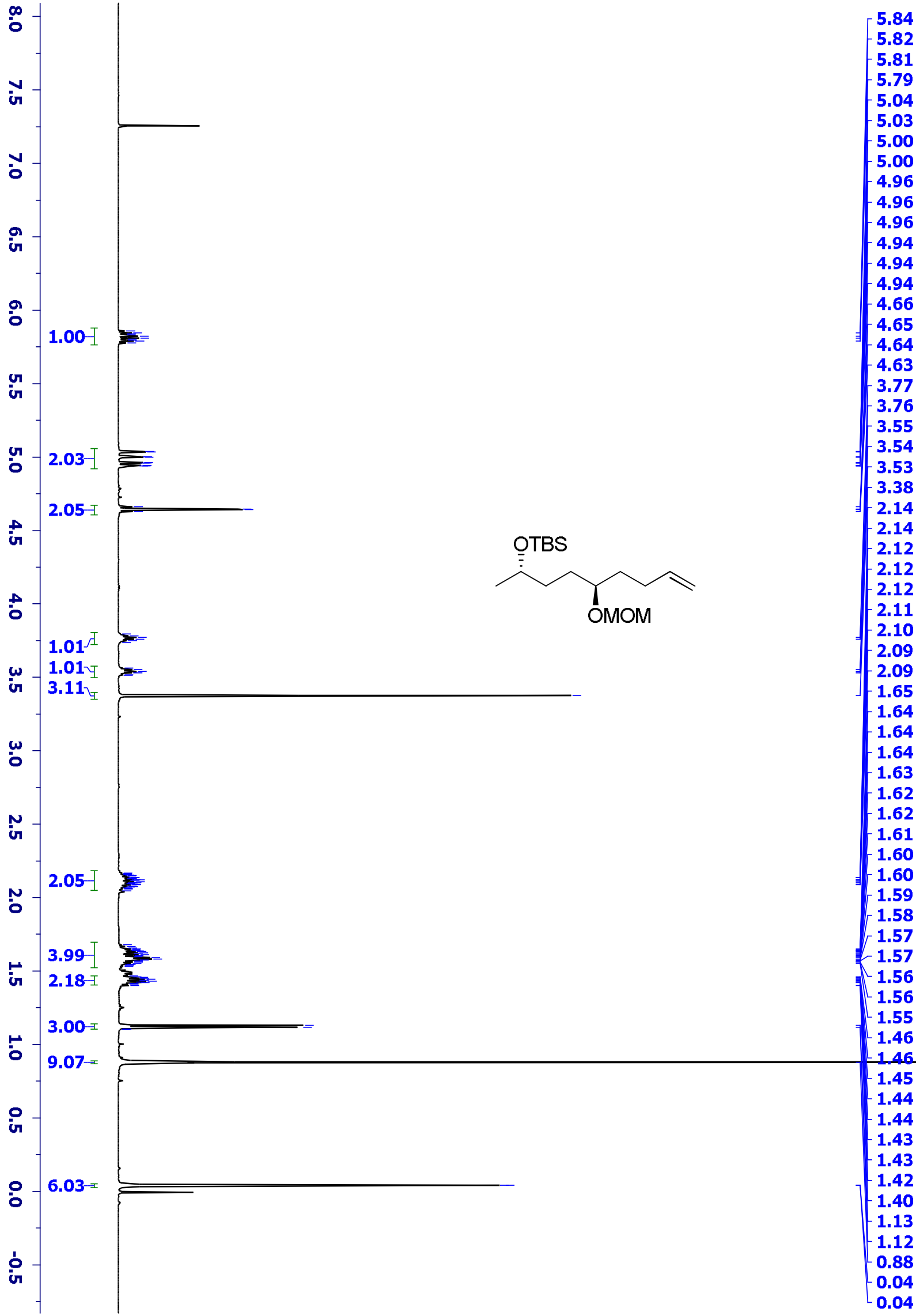

${ }^{1} \mathrm{H}$ NMR spectrum of $\mathbf{1 9 a}\left(500 \mathrm{MHz}, \mathrm{CDCl}_{3}\right)$ 


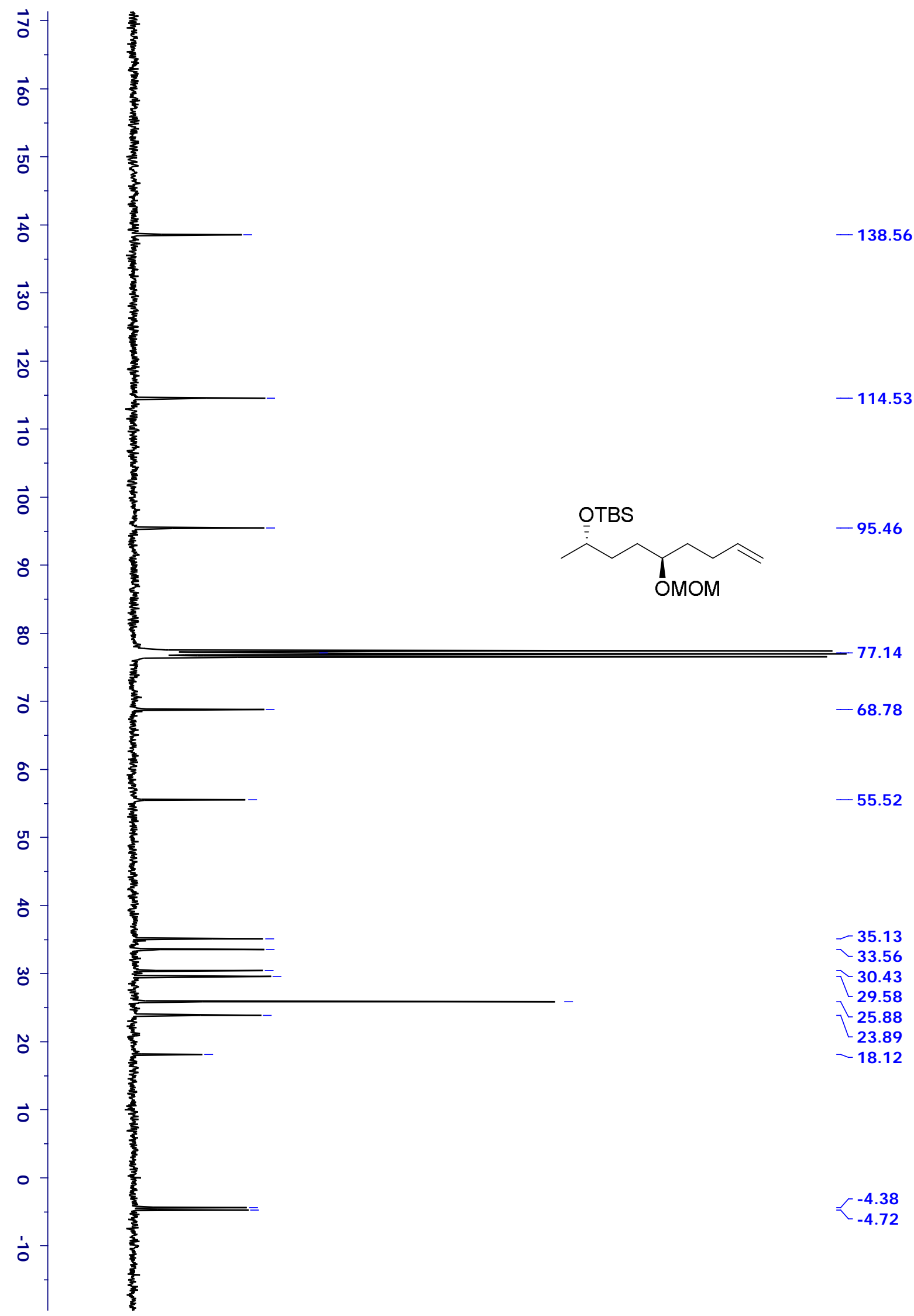

${ }^{13} \mathrm{C}$ NMR spectrum of $\mathbf{1 9 a}\left(100 \mathrm{MHz}, \mathrm{CDCl}_{3}\right)$ 


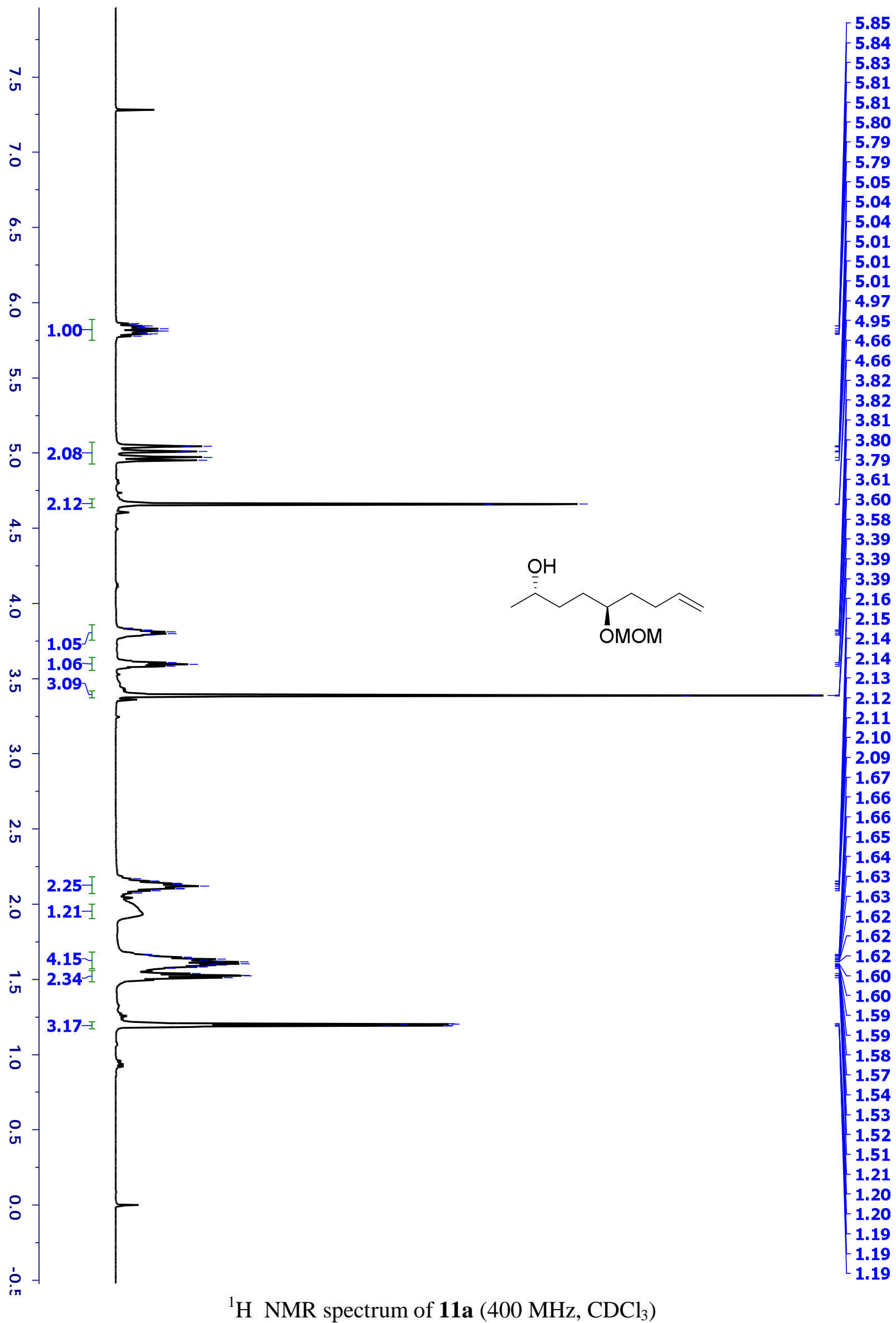




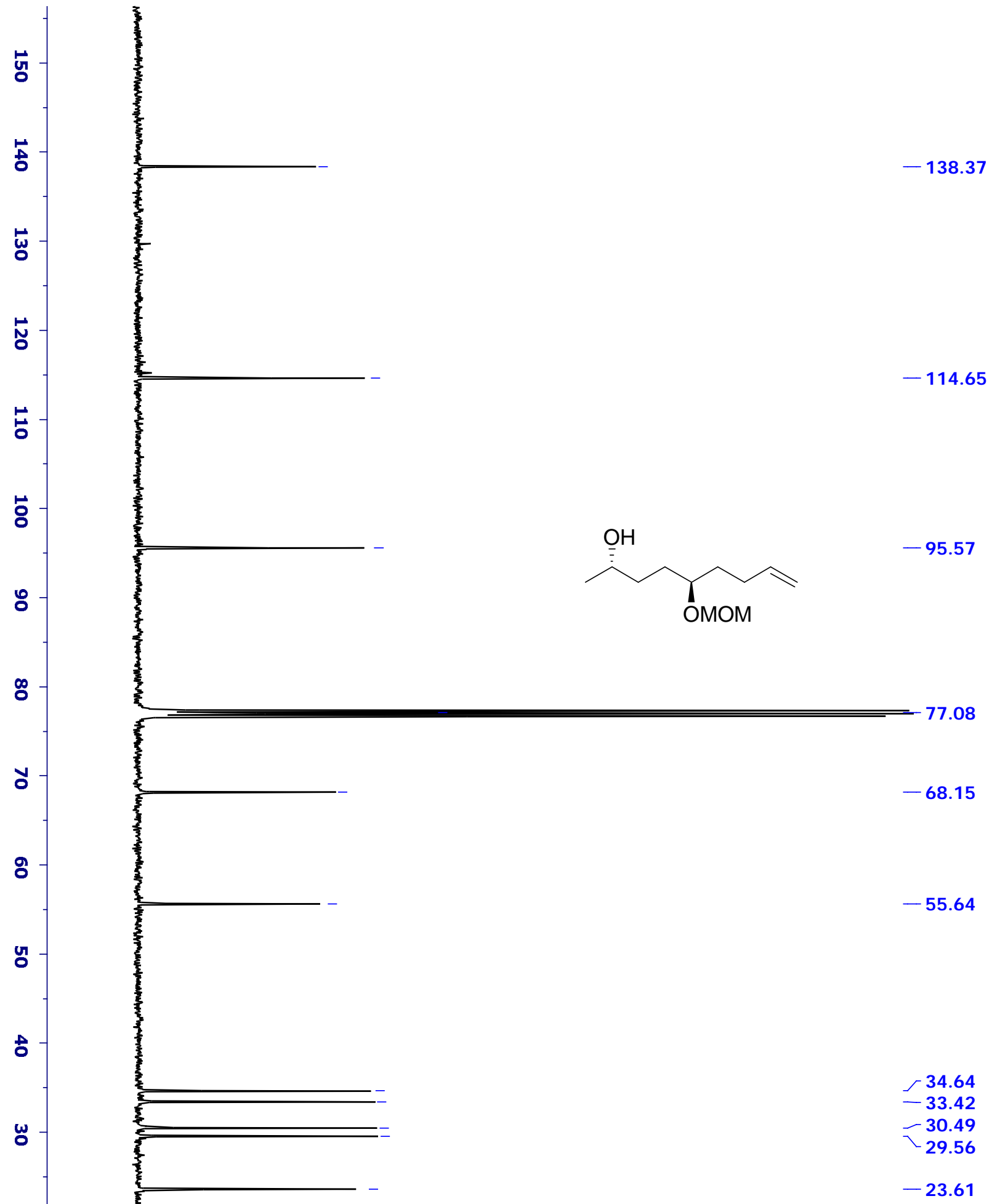

${ }^{13} \mathrm{C}$ NMR spectrum of $11 a\left(100 \mathrm{MHz}, \mathrm{CDCl}_{3}\right)$ 


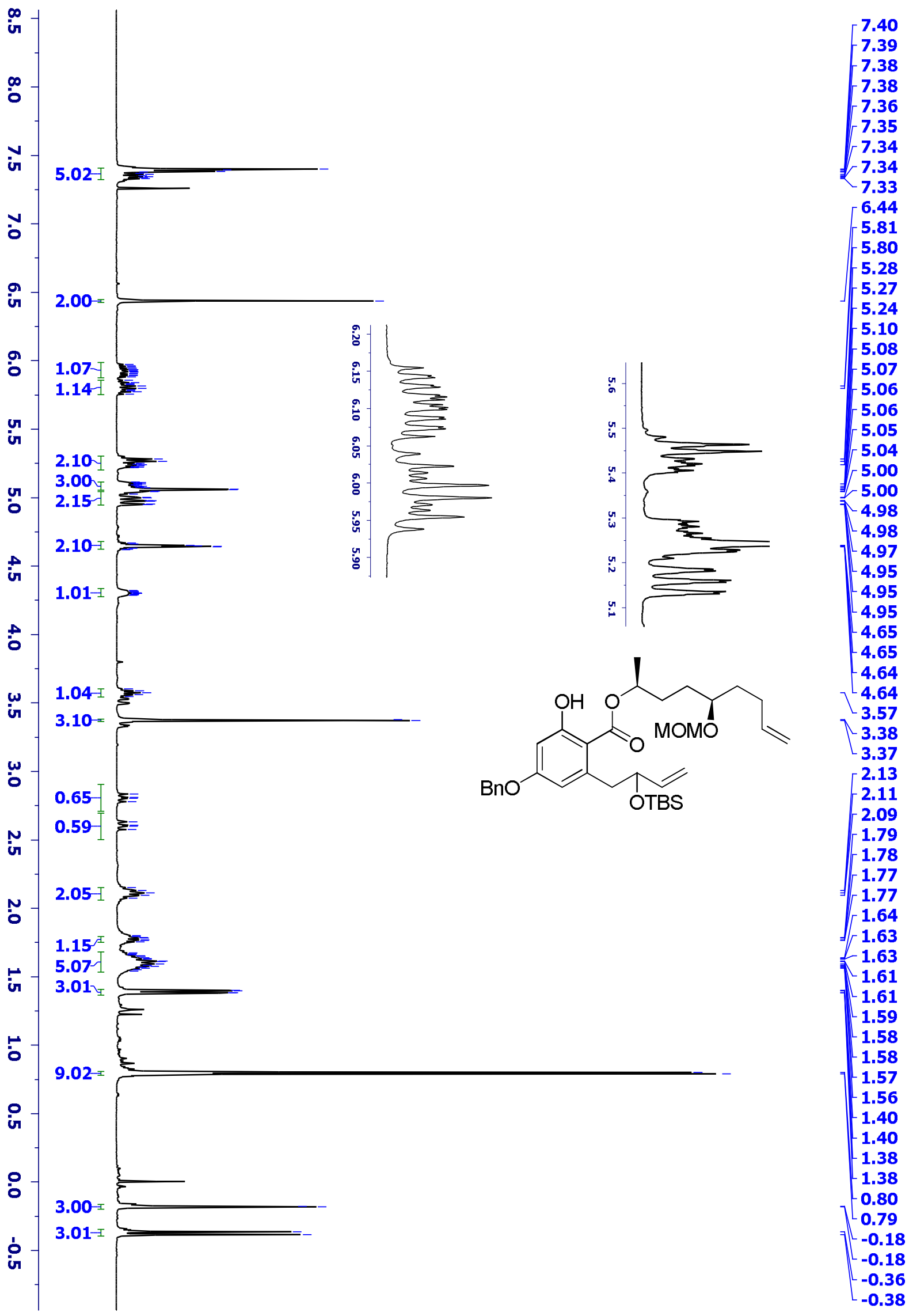

${ }^{1} \mathrm{H}$ NMR spectrum of $\mathbf{2 2 a}\left(400 \mathrm{MHz}, \mathrm{CDCl}_{3}\right)$ 


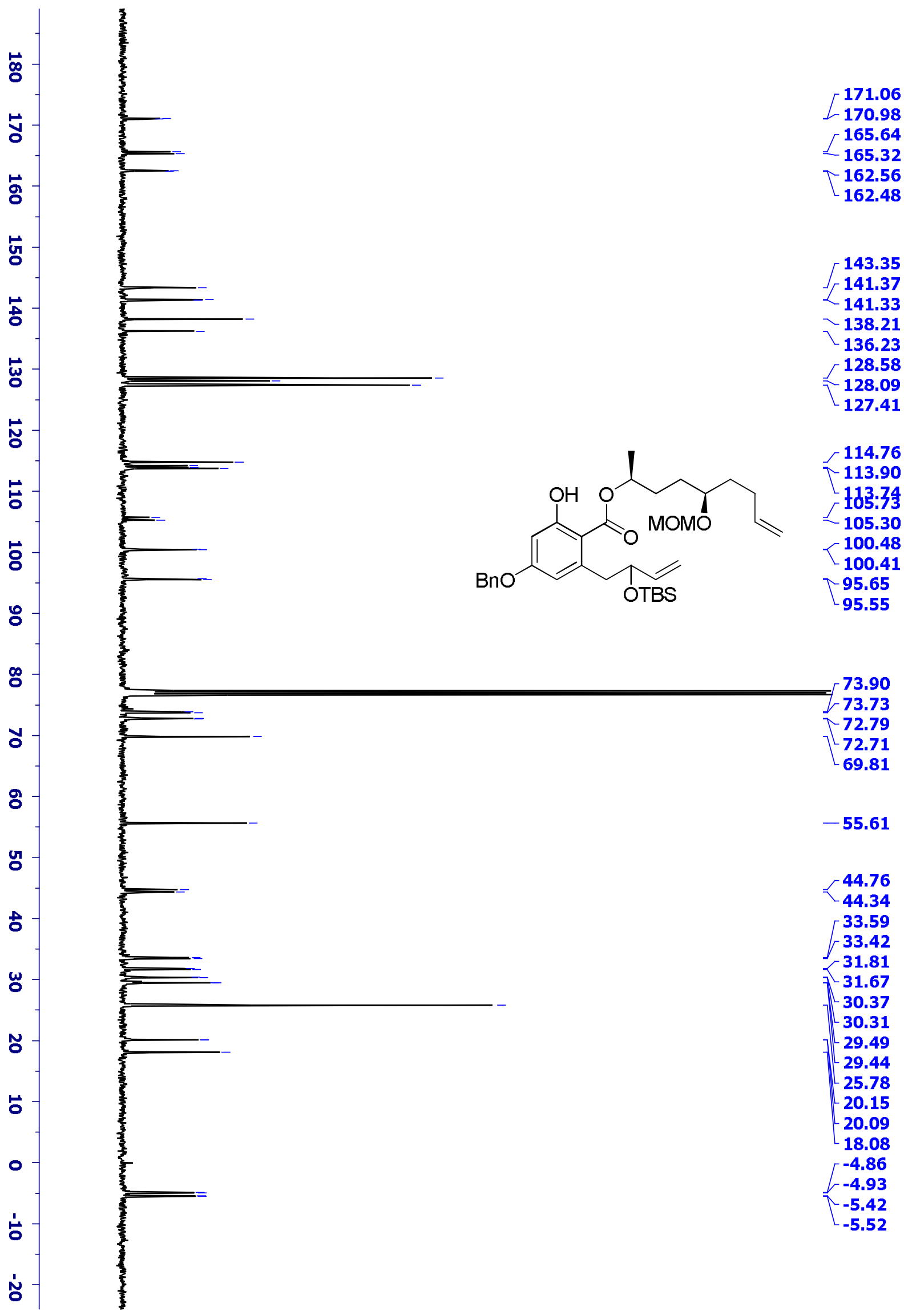

${ }^{13} \mathrm{C}$ NMR spectrum of $22 \mathbf{a}\left(100 \mathrm{MHz}, \mathrm{CDCl}_{3}\right)$ 


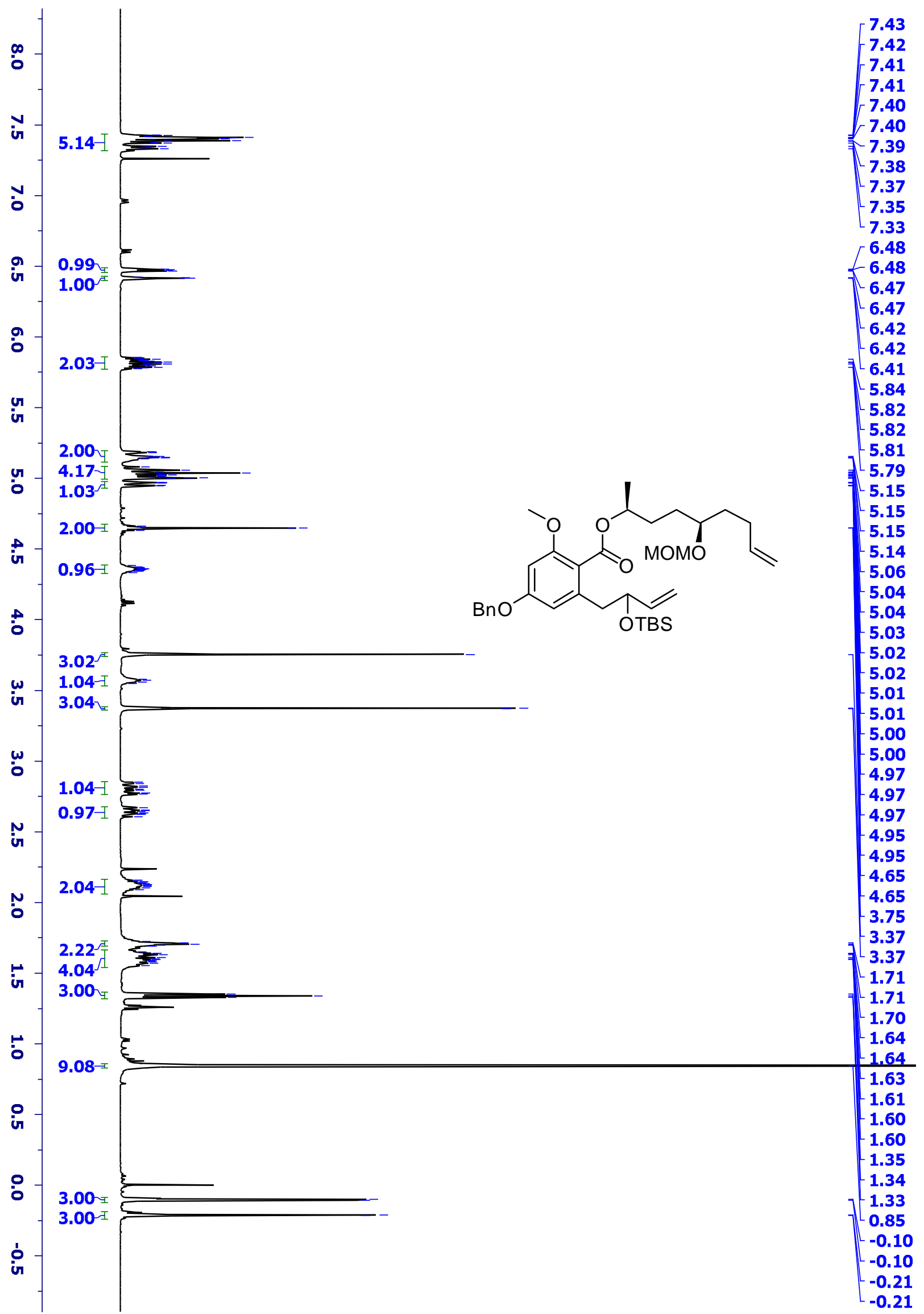

${ }^{1} \mathrm{H}$ NMR spectrum of 23a(500 MHz, $\left.\mathrm{CDCl}_{3}\right)$ 


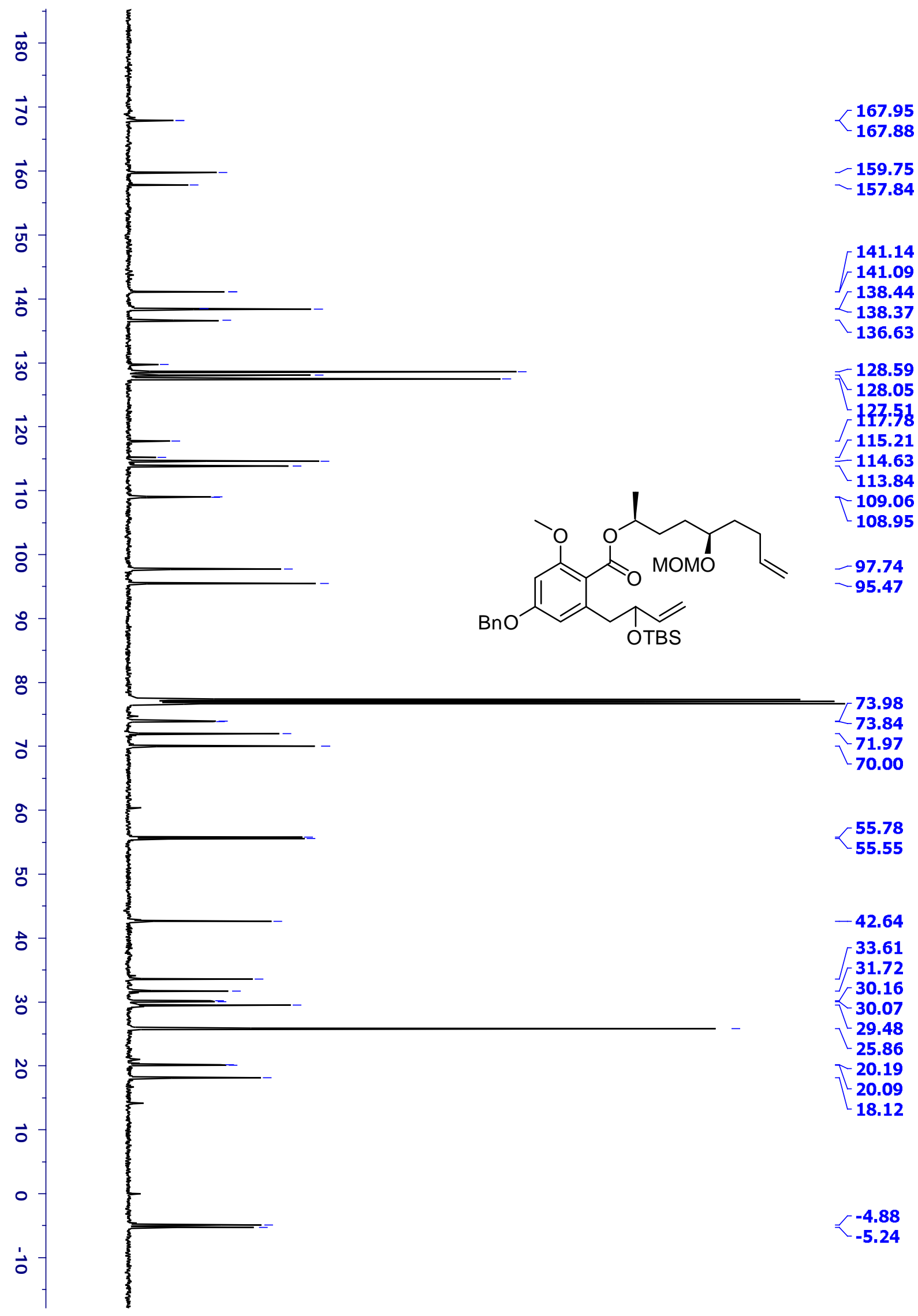

${ }^{13} \mathrm{C}$ NMR spectrum of $23 \mathbf{a}\left(100 \mathrm{MHz}, \mathrm{CDCl}_{3}\right)$ 


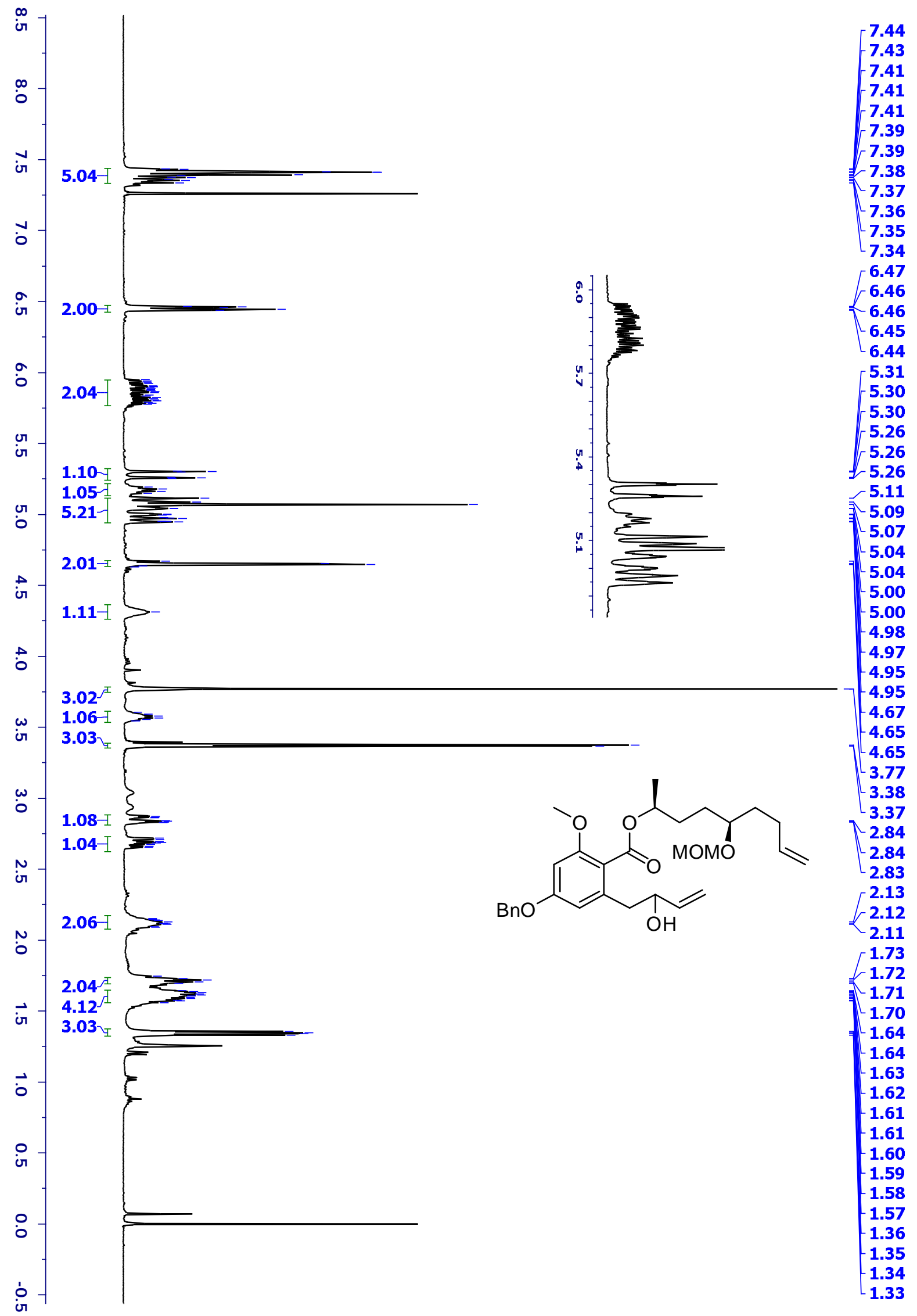

${ }^{1} \mathrm{H}$ NMR spectrum of $\mathbf{2 4 a}\left(400 \mathrm{MHz}, \mathrm{CDCl}_{3}\right)$ 


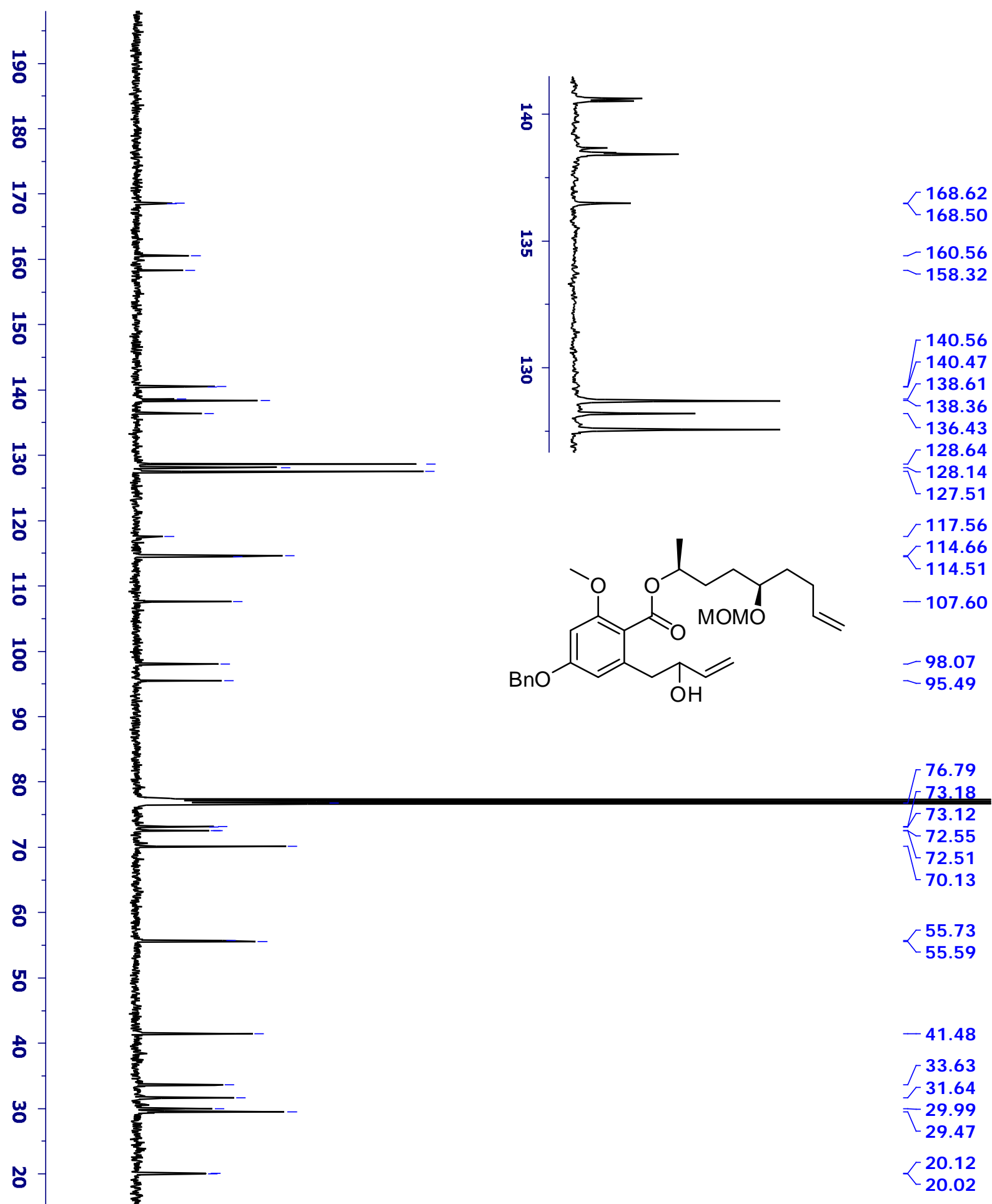

${ }^{13} \mathrm{C}$ NMR spectrum of $\mathbf{2 4 a}\left(100 \mathrm{MHz}, \mathrm{CDCl}_{3}\right)$ 


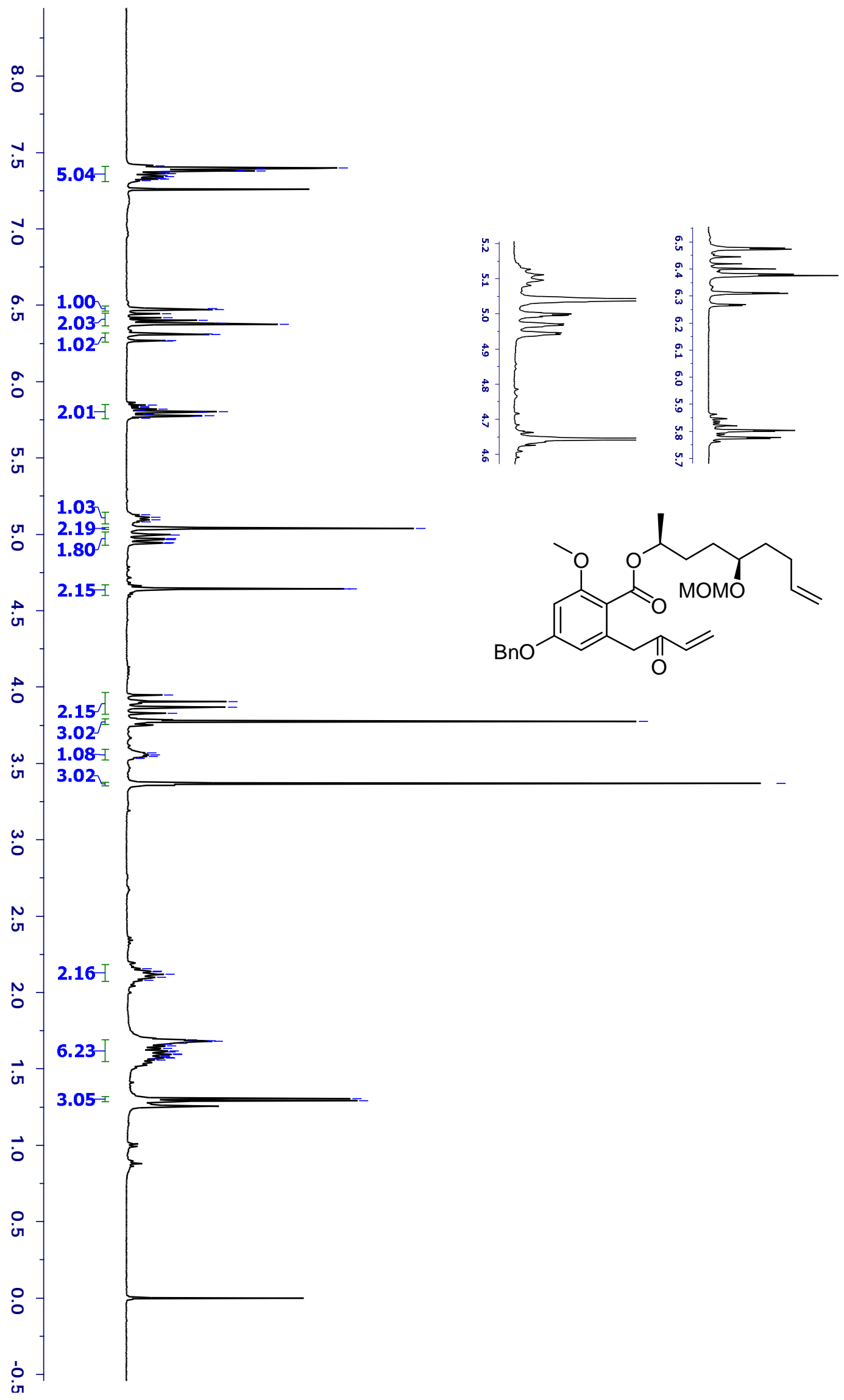

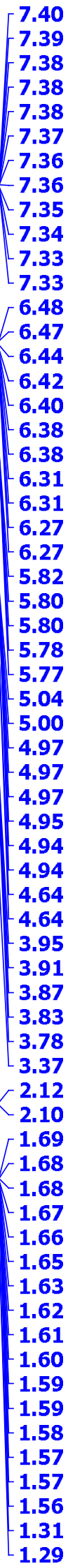

${ }^{1} \mathrm{H}$ NMR spectrum of $\mathbf{9 a}\left(400 \mathrm{MHz}, \mathrm{CDCl}_{3}\right)$ 


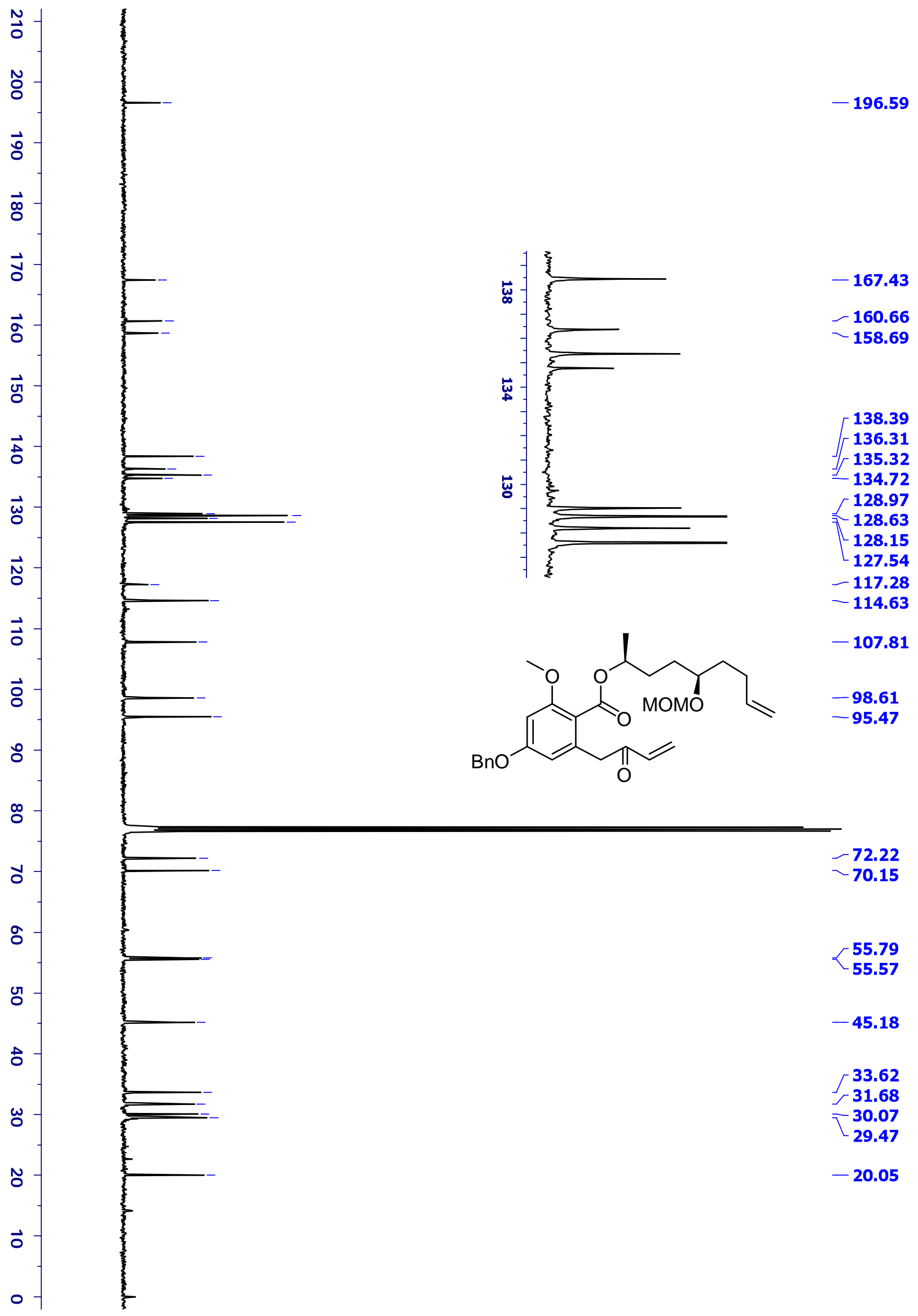

${ }^{13} \mathrm{C}$ NMR spectrum of 9a $\left(100 \mathrm{MHz}, \mathrm{CDCl}_{3}\right)$ 


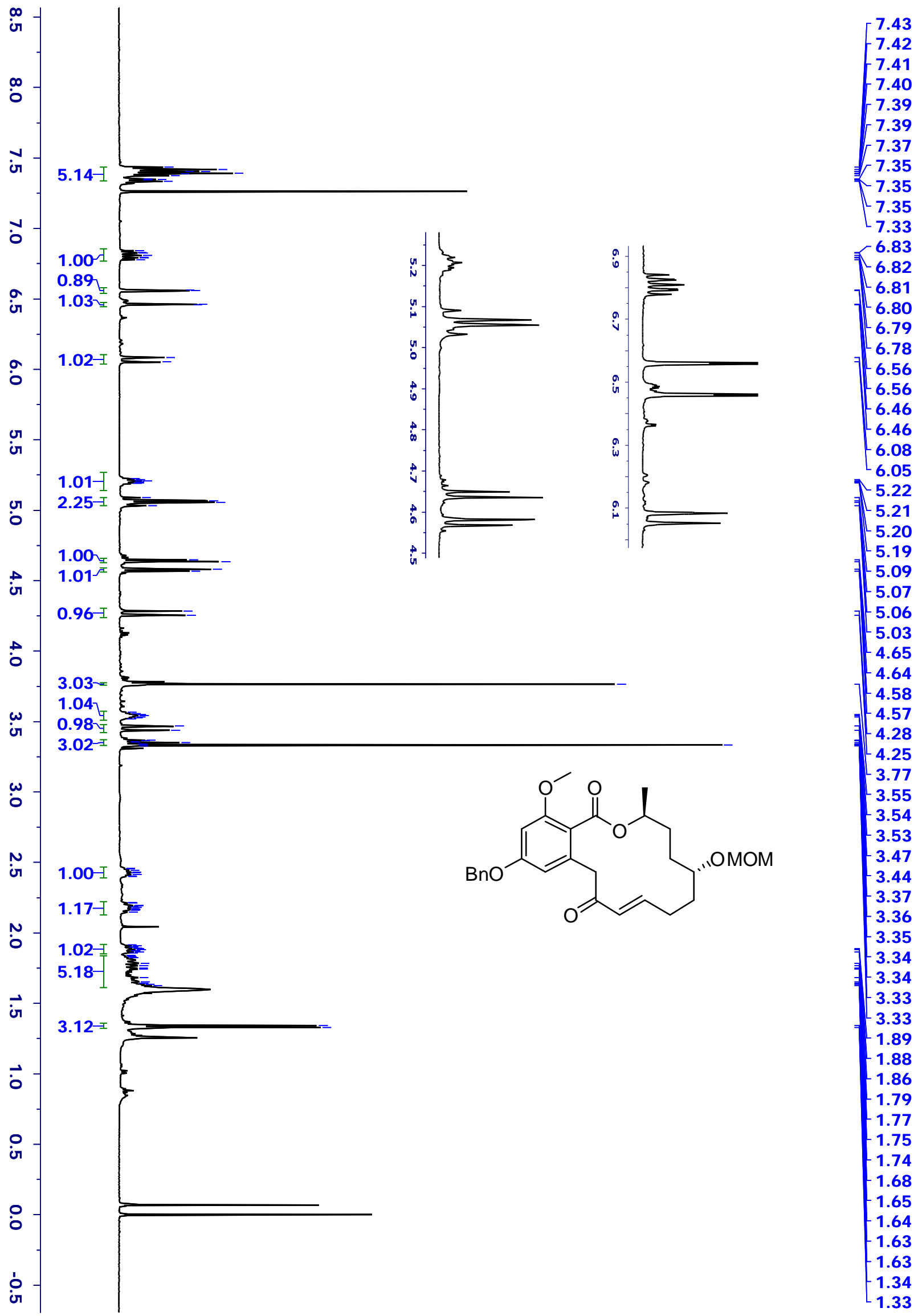

${ }^{1} \mathrm{H}$ NMR spectrum of $7 \mathbf{a}\left(500 \mathrm{MHz}, \mathrm{CDCl}_{3}\right)$ 


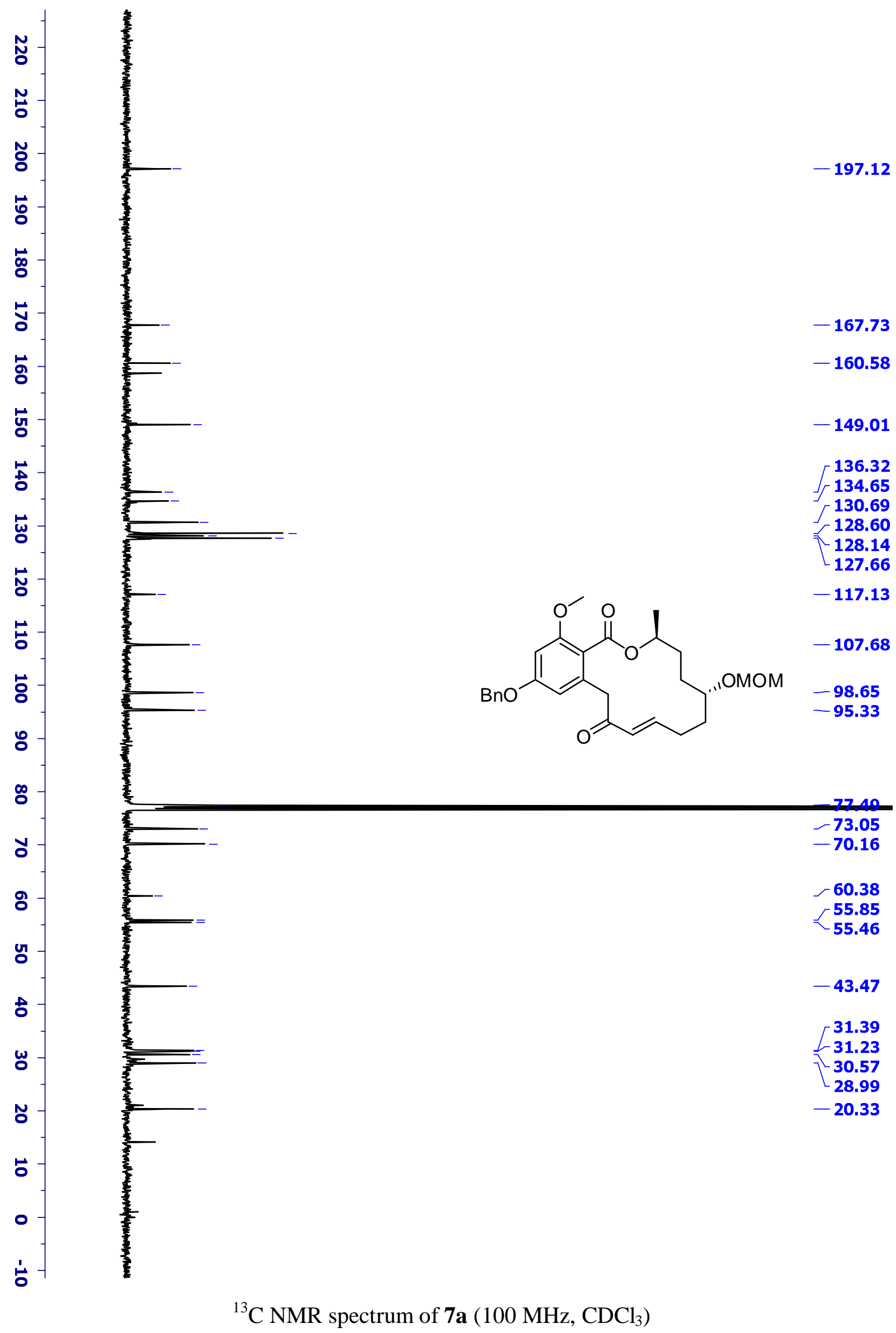




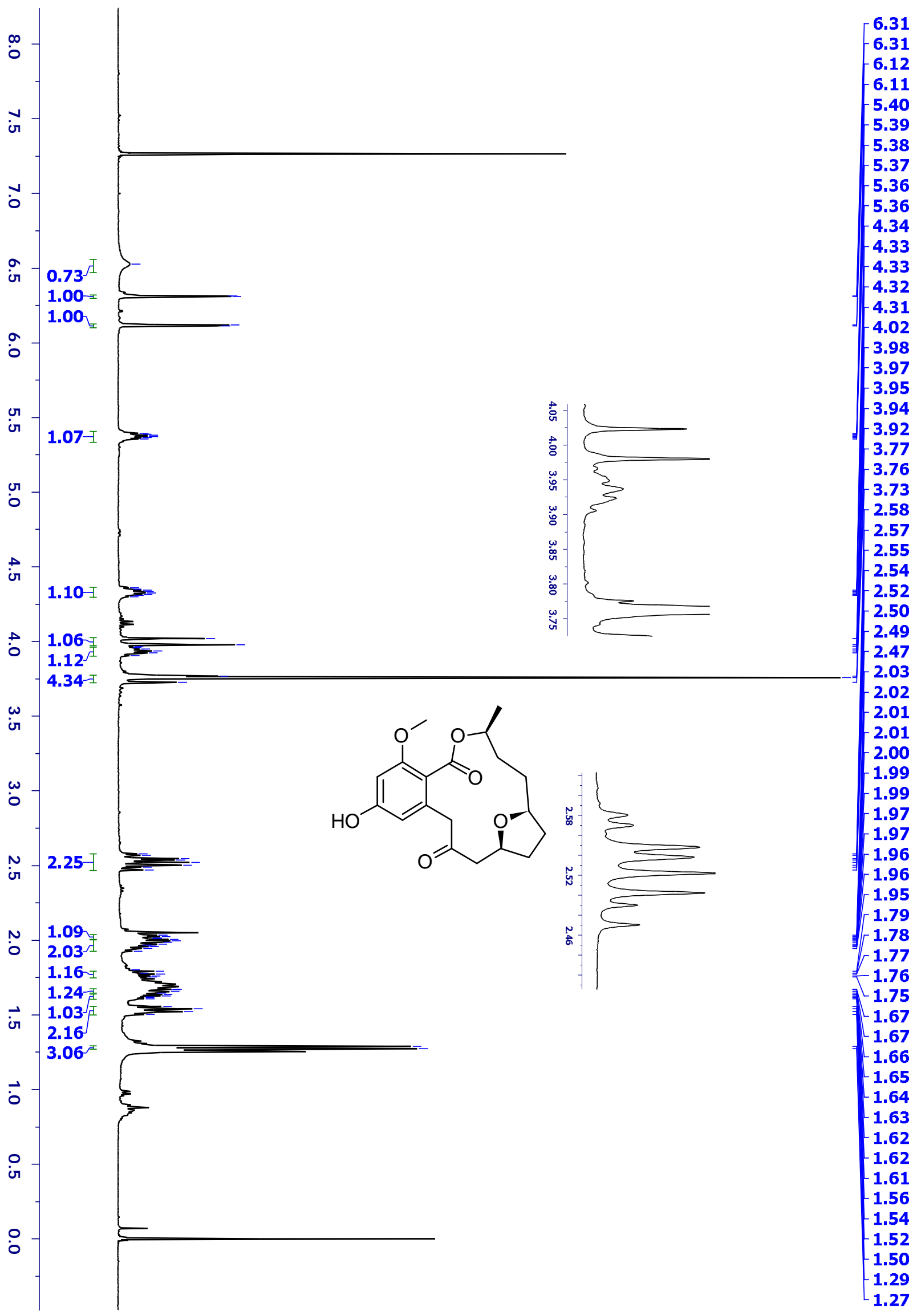

${ }^{1} \mathrm{H}$ NMR spectrum of $4\left(400 \mathrm{MHz}, \mathrm{CDCl}_{3}\right)$ 


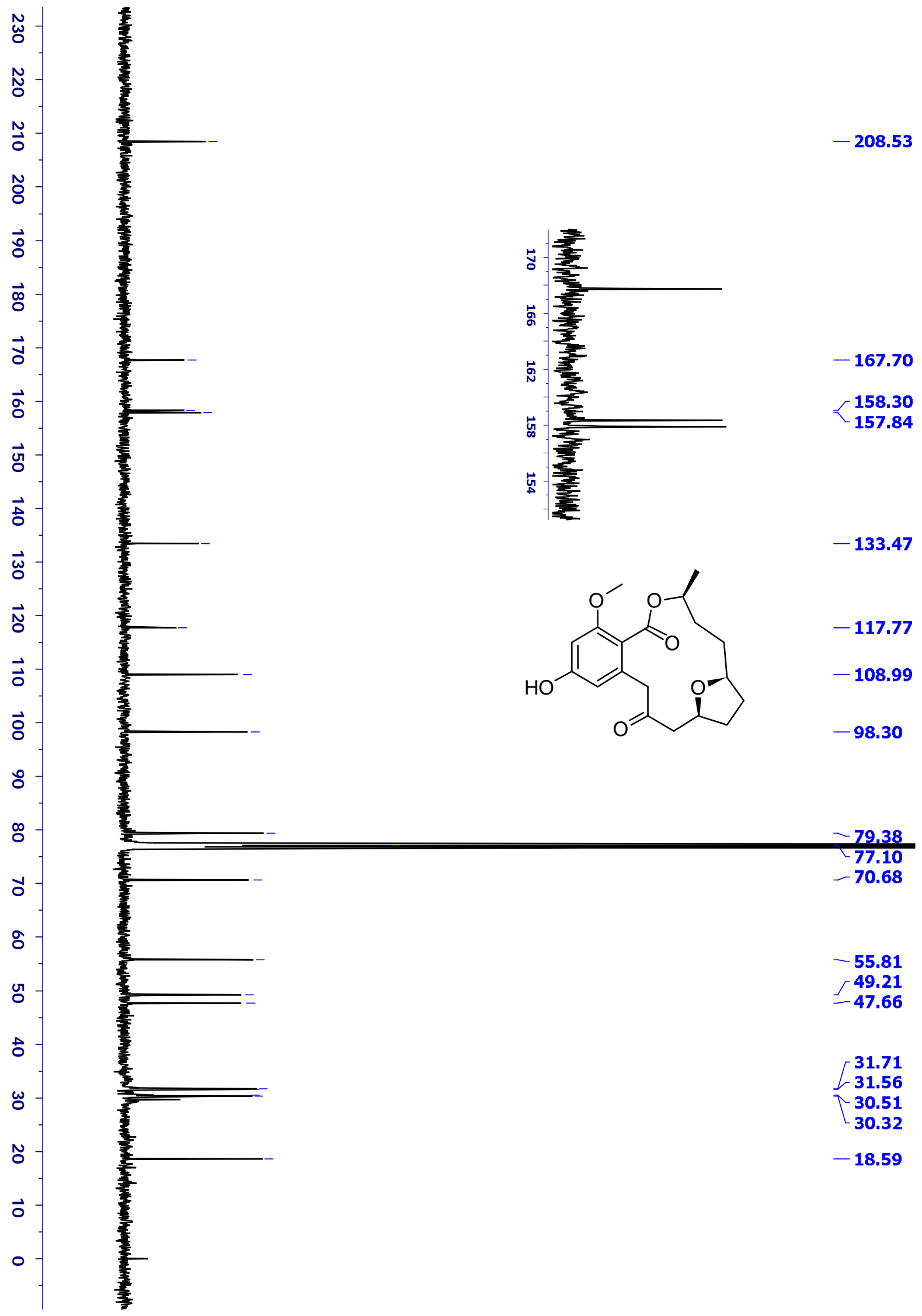

${ }^{13} \mathrm{C}$ NMR spectrum of $4\left(100 \mathrm{MHz}, \mathrm{CDCl}_{3}\right)$ 


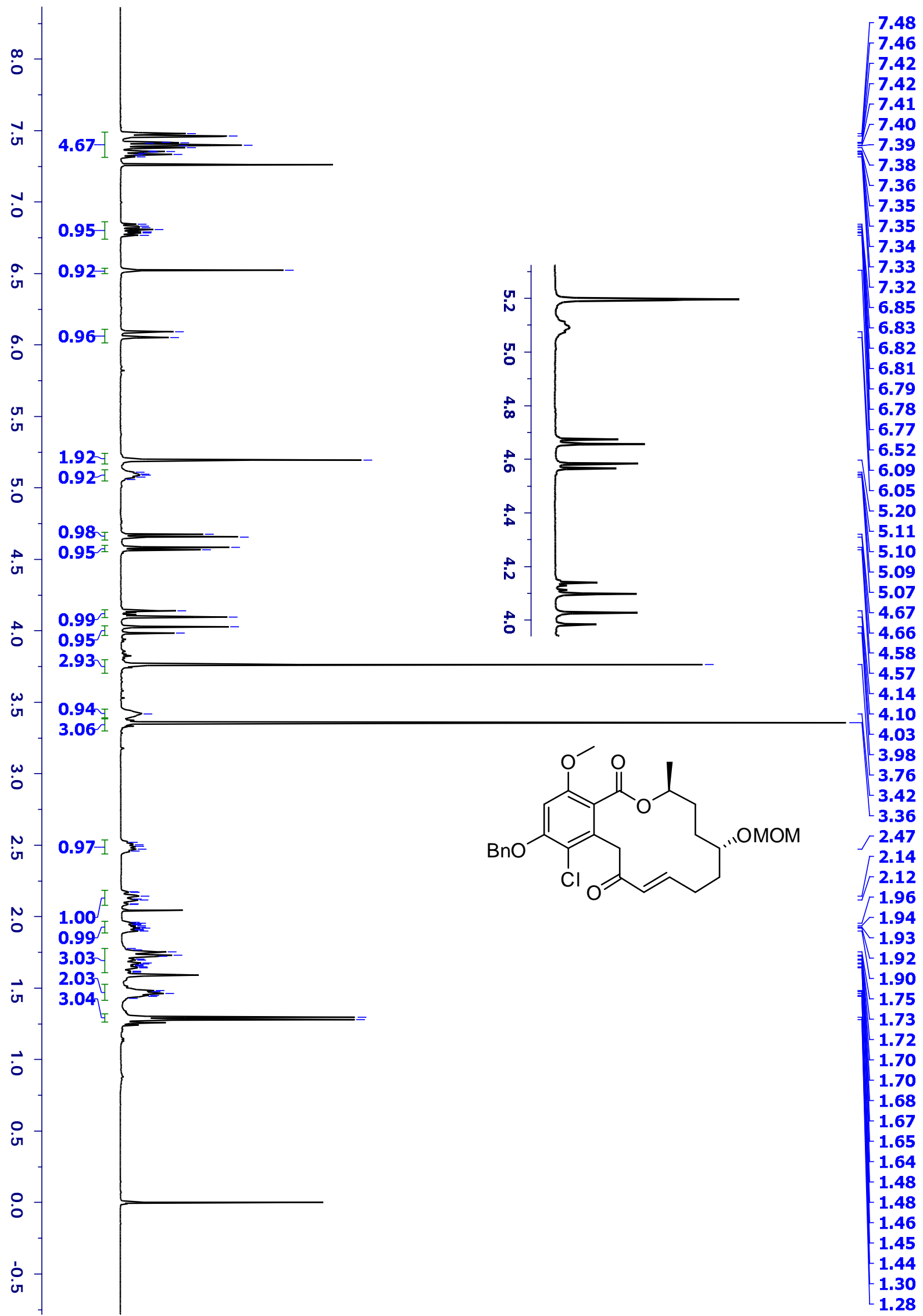

${ }^{1} \mathrm{H}$ NMR spectrum of $\mathbf{8 a}\left(400 \mathrm{MHz}, \mathrm{CDCl}_{3}\right)$ 


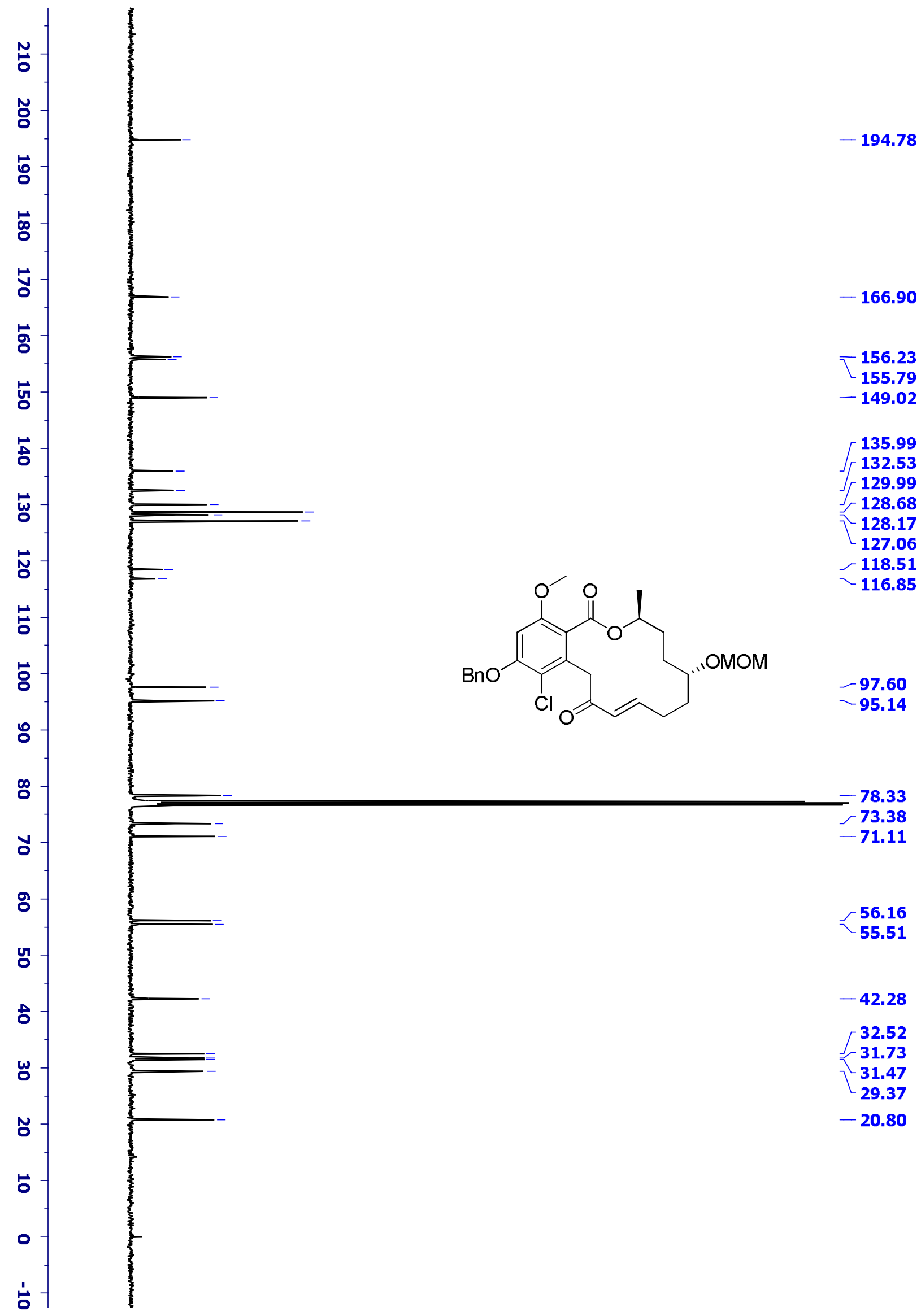

${ }^{13} \mathrm{C}$ NMR spectrum of $\mathbf{8 a}\left(100 \mathrm{MHz}, \mathrm{CDCl}_{3}\right)$ 


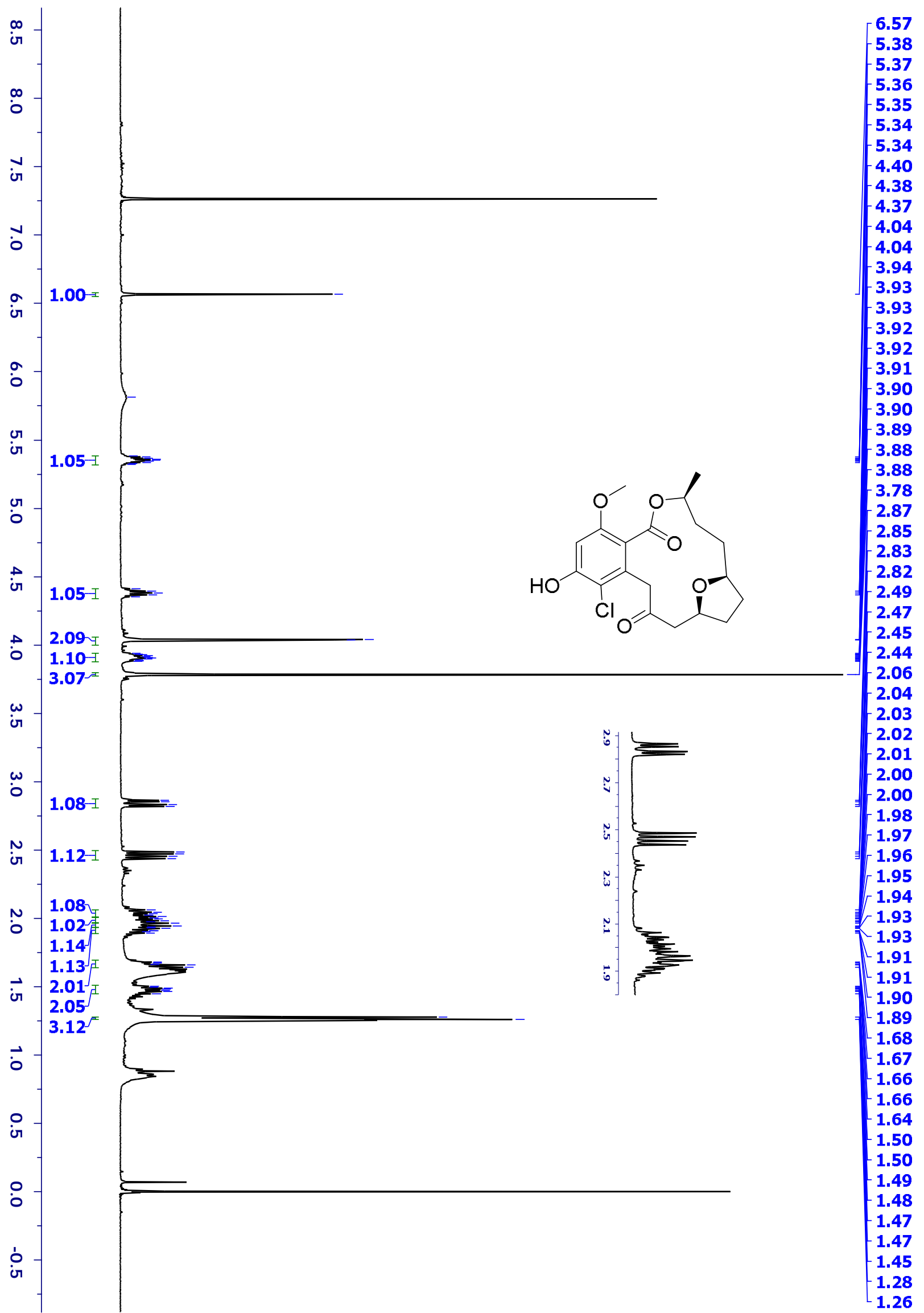

${ }^{1} \mathrm{H}$ NMR spectrum of 5 (400 MHz, $\mathrm{CDCl}_{3}$ ) 


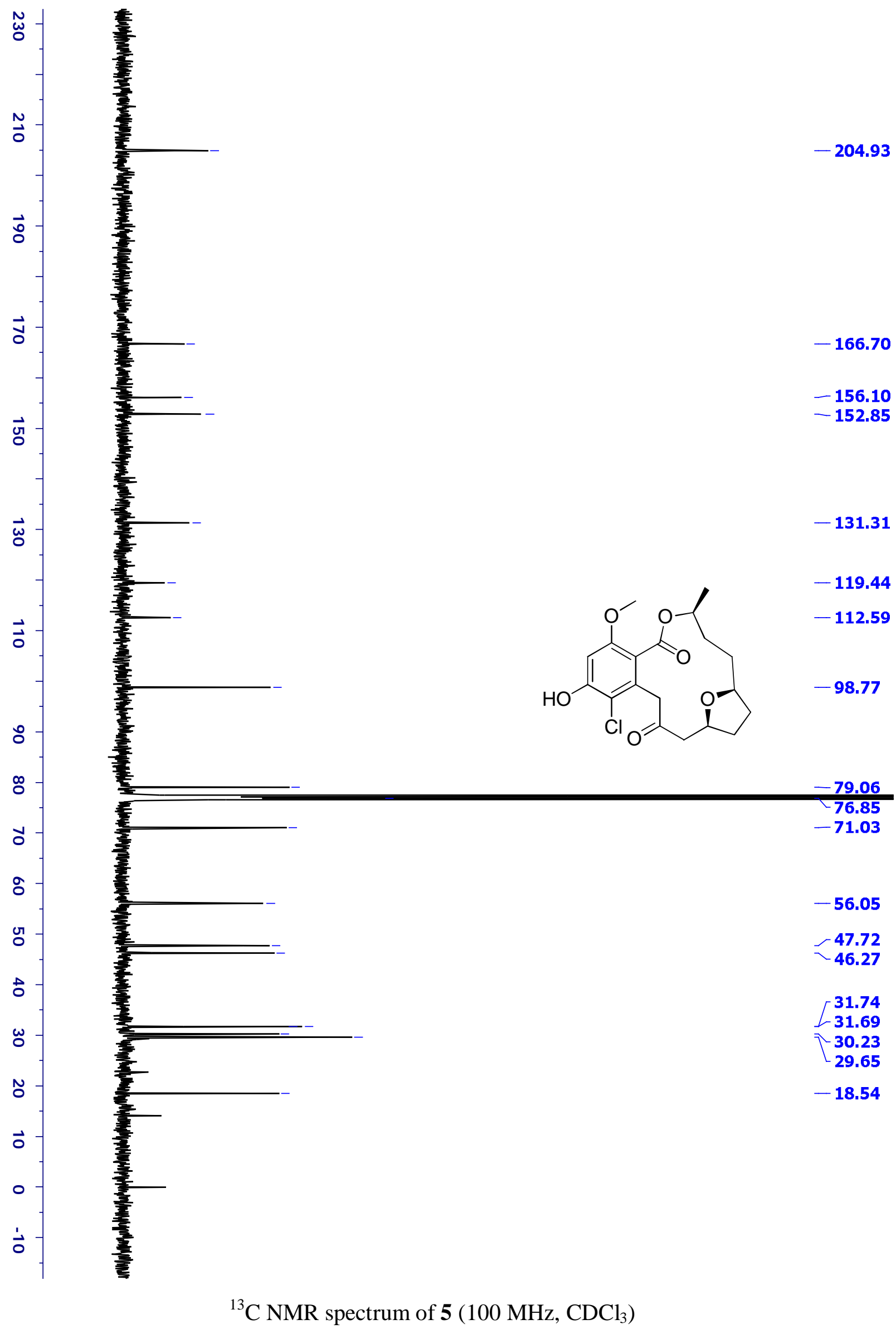




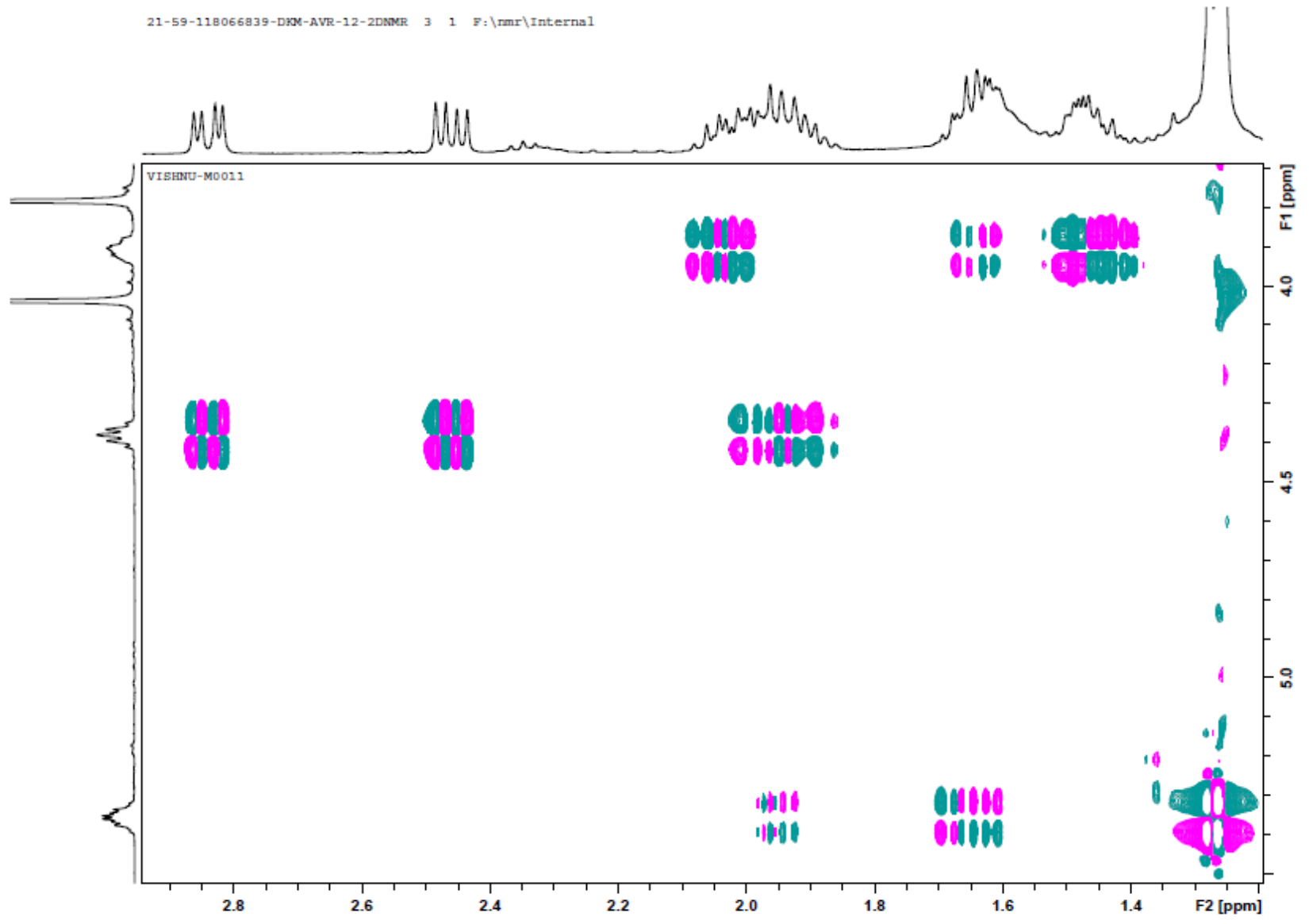

Exapnded version of the DQF-COSY Spectrum of $4\left(400 \mathrm{MHz}, \mathrm{CDCl}_{3}\right)$

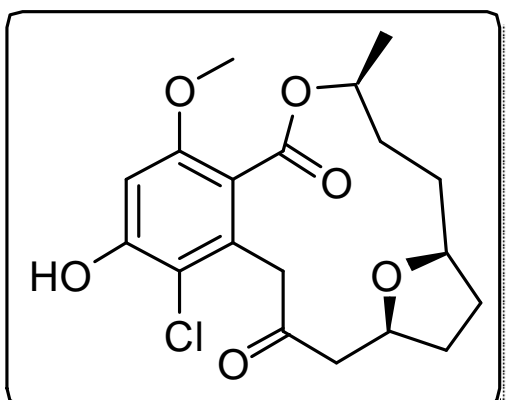




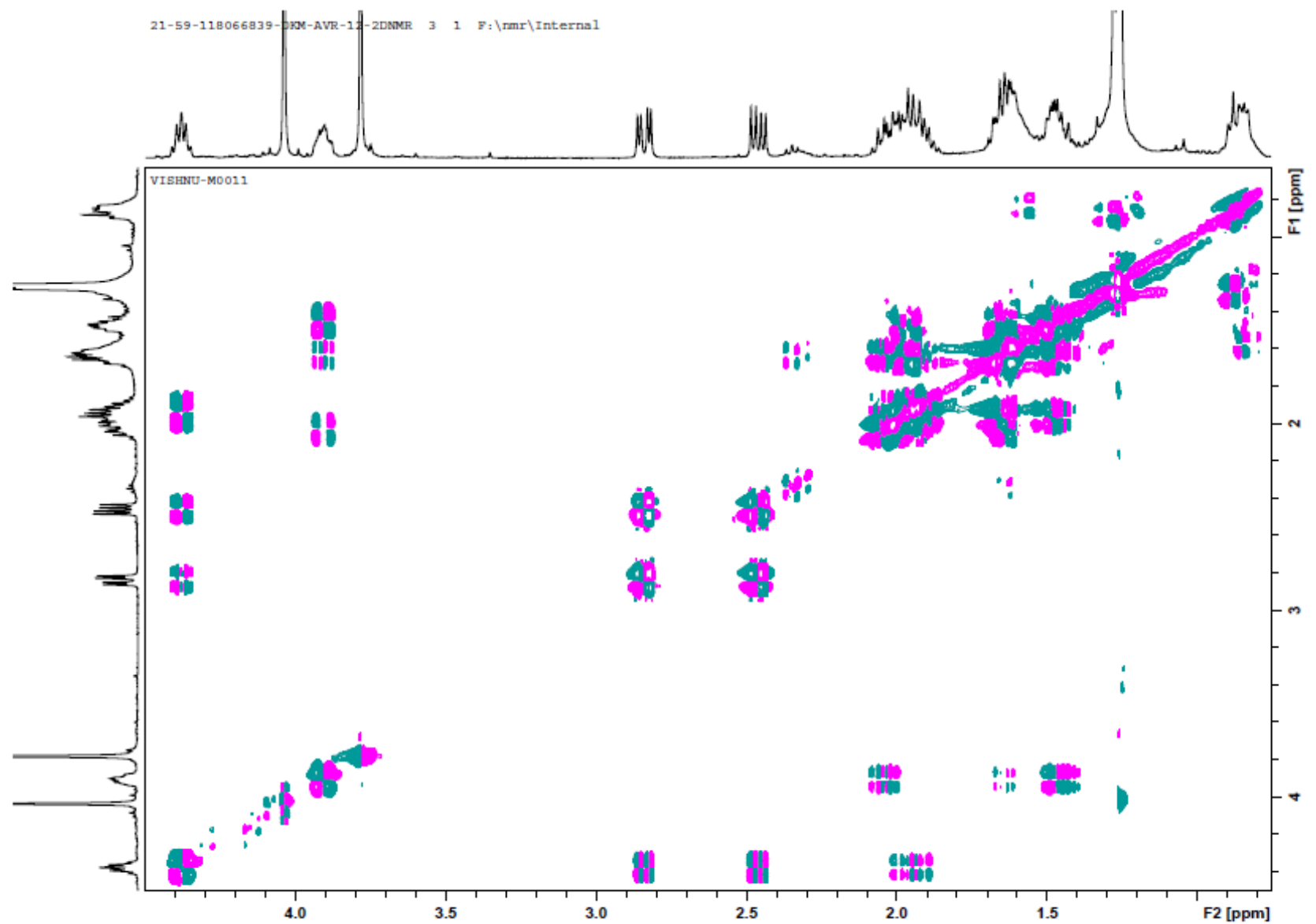

Exapnded version of the DQF-COSY Spectrum of $4\left(400 \mathrm{MHz}, \mathrm{CDCl}_{3}\right)$

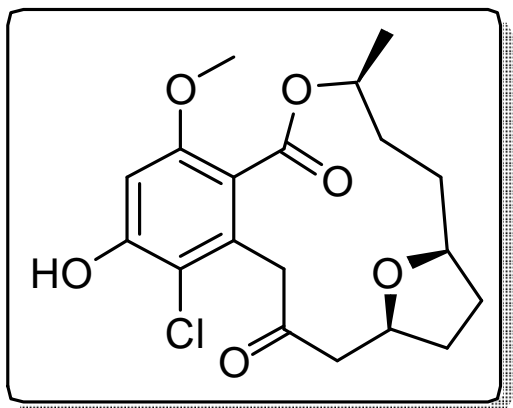




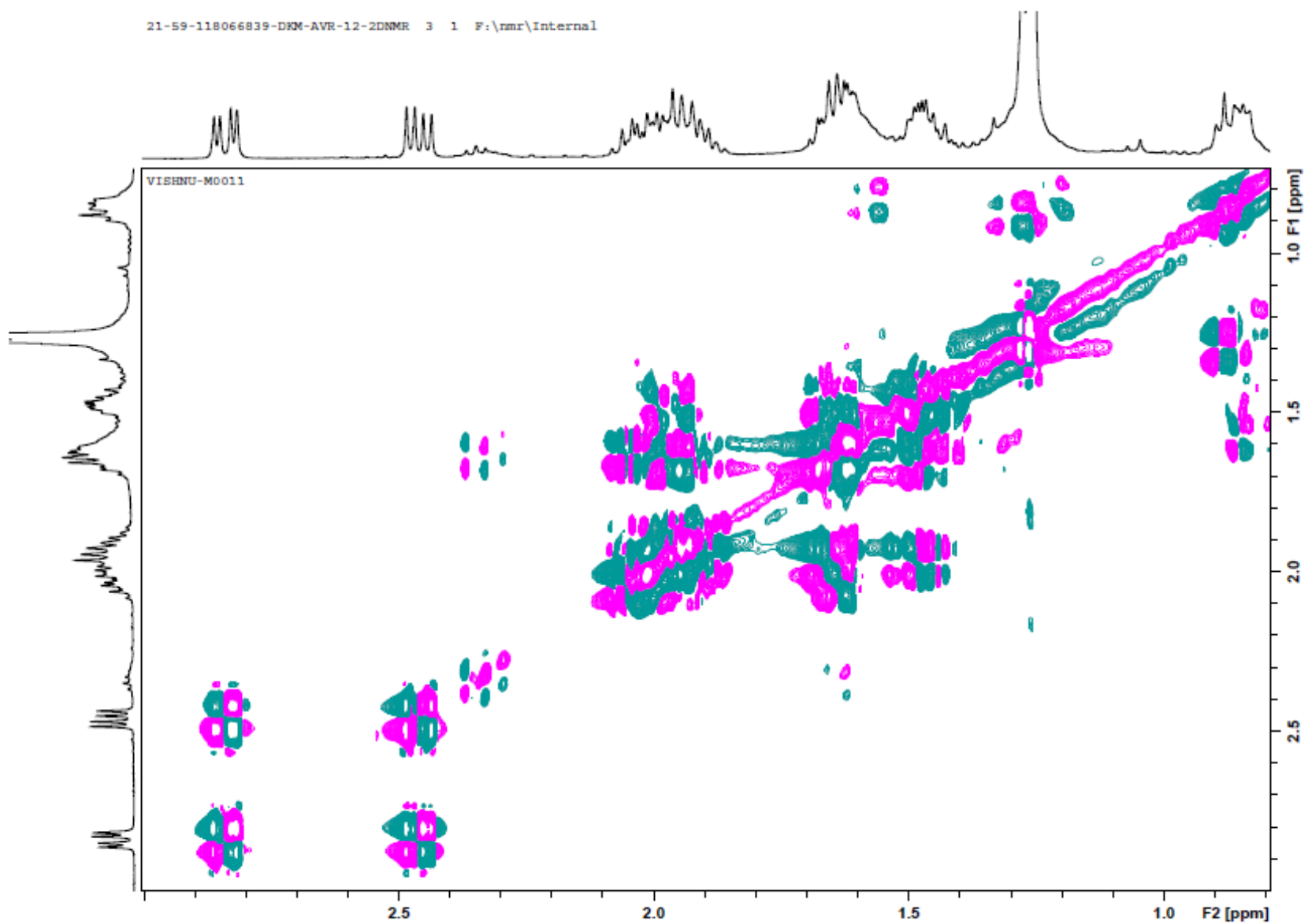

Exapnded version of the DQF-COSY Spectrum of $5\left(400 \mathrm{MHz}, \mathrm{CDCl}_{3}\right)$

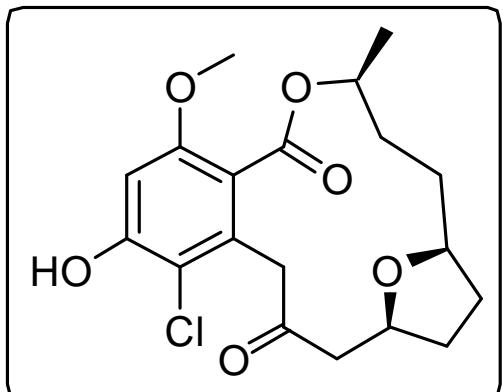




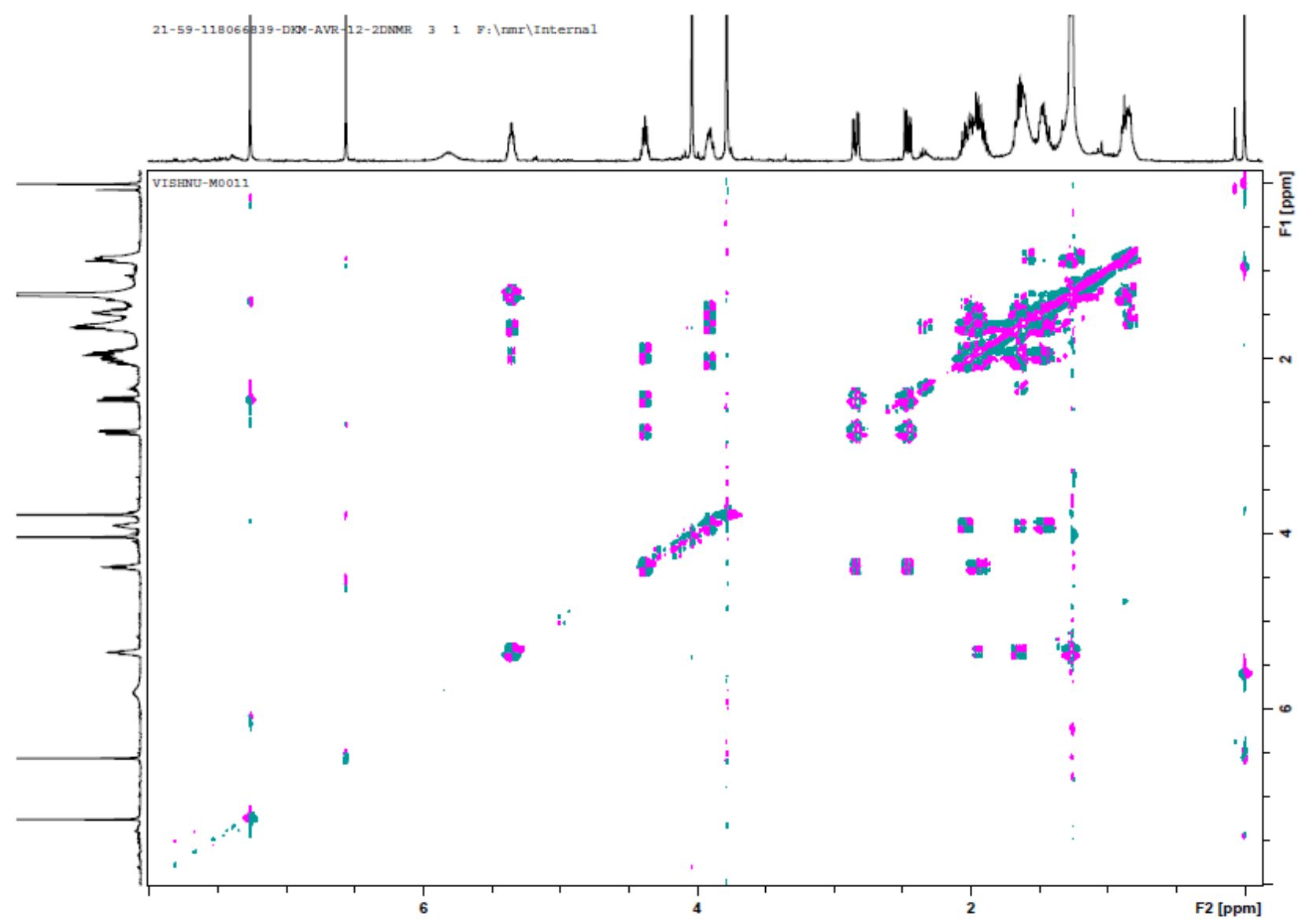

DQF-COSY Spectrum of $5\left(400 \mathrm{MHz}, \mathrm{CDCl}_{3}\right)$

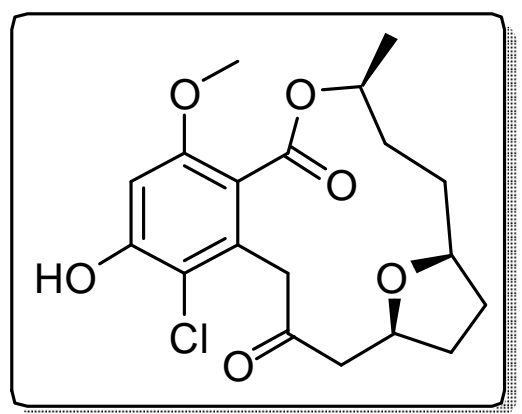




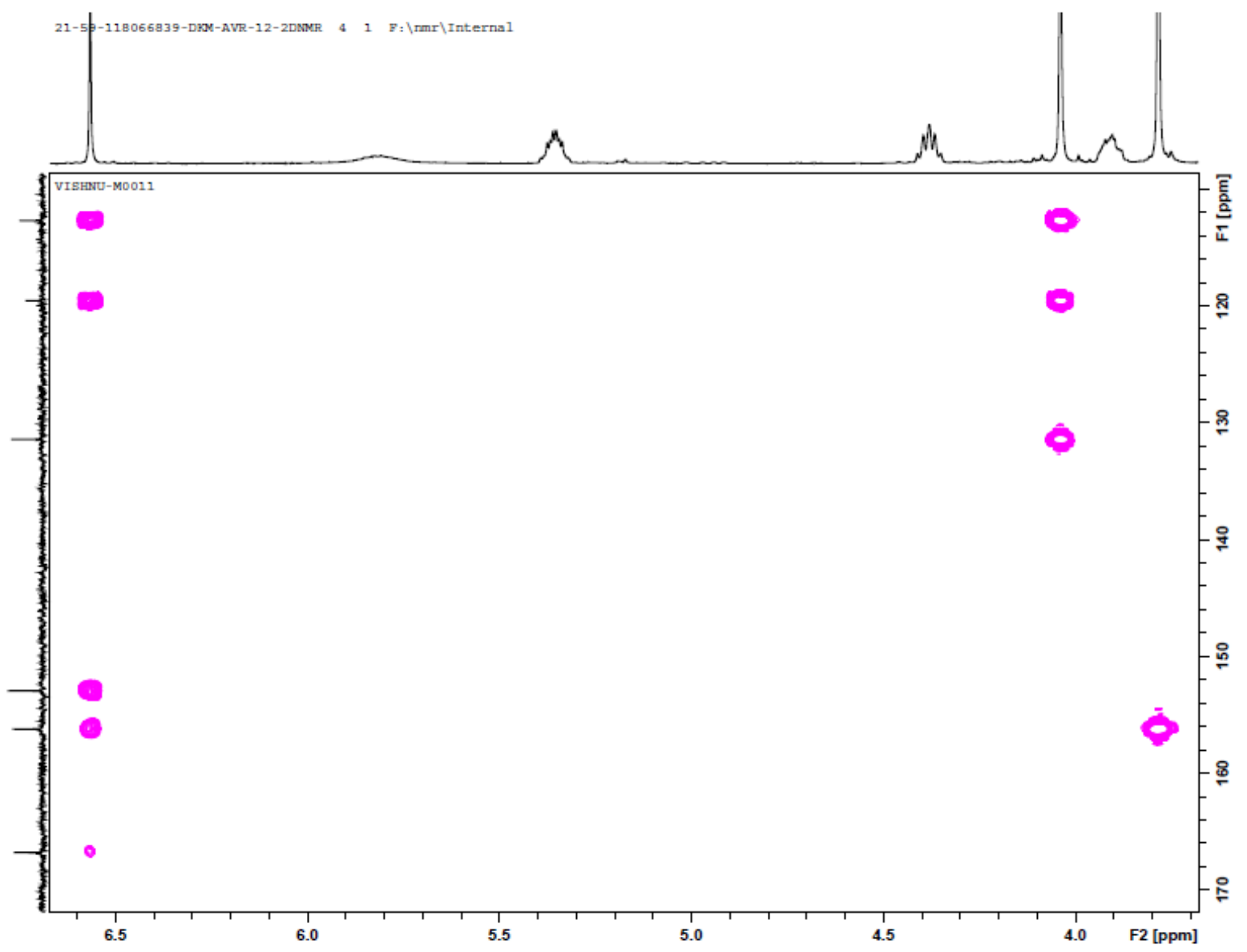

Expanded version of ${ }^{13} \mathrm{C}-{ }^{1} \mathrm{H} \mathrm{HMBC}$ spectrum of compound $5\left(400 \mathrm{MHz}, \mathrm{CDCl}_{3}\right.$ )<smiles>COc1cc(O)c(Cl)c(CC(=O)C[C@@H]2CC[C@H]3CC[C@H]2CC3)c1C(=O)O[C@H]1CCCO1</smiles> 


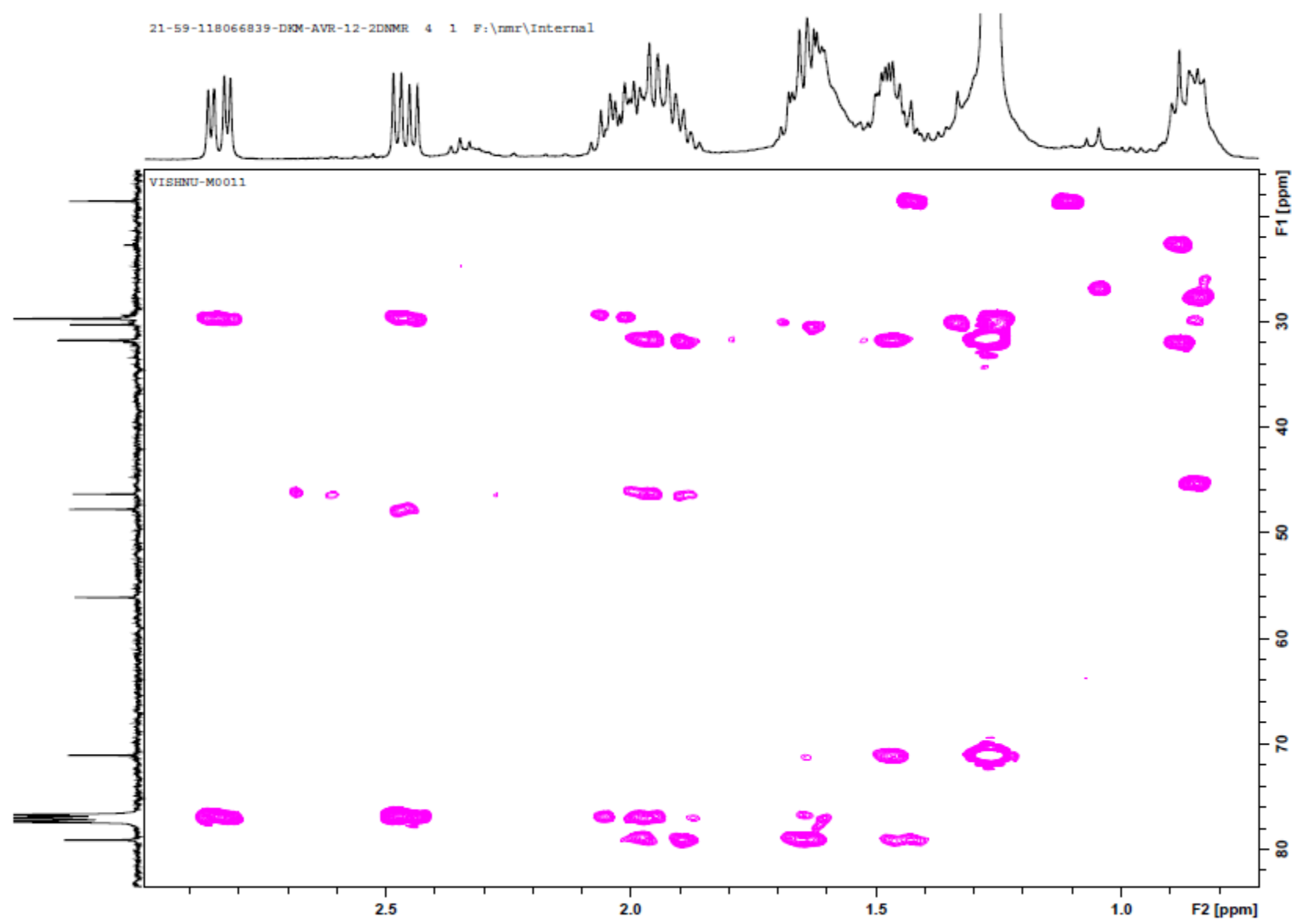

Expanded version of ${ }^{13} \mathrm{C}-{ }^{1} \mathrm{H}$ HMBC spectrum of compound 5 (400 MHz, $\mathrm{CDCl}_{3}$ )

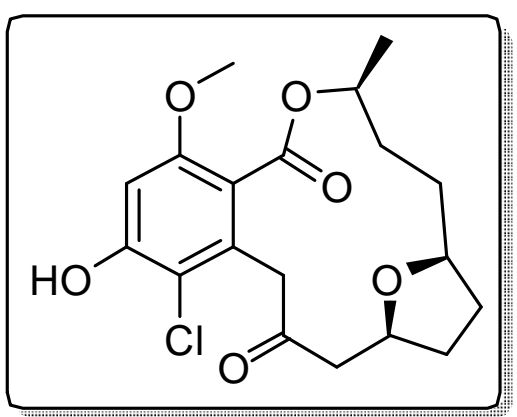




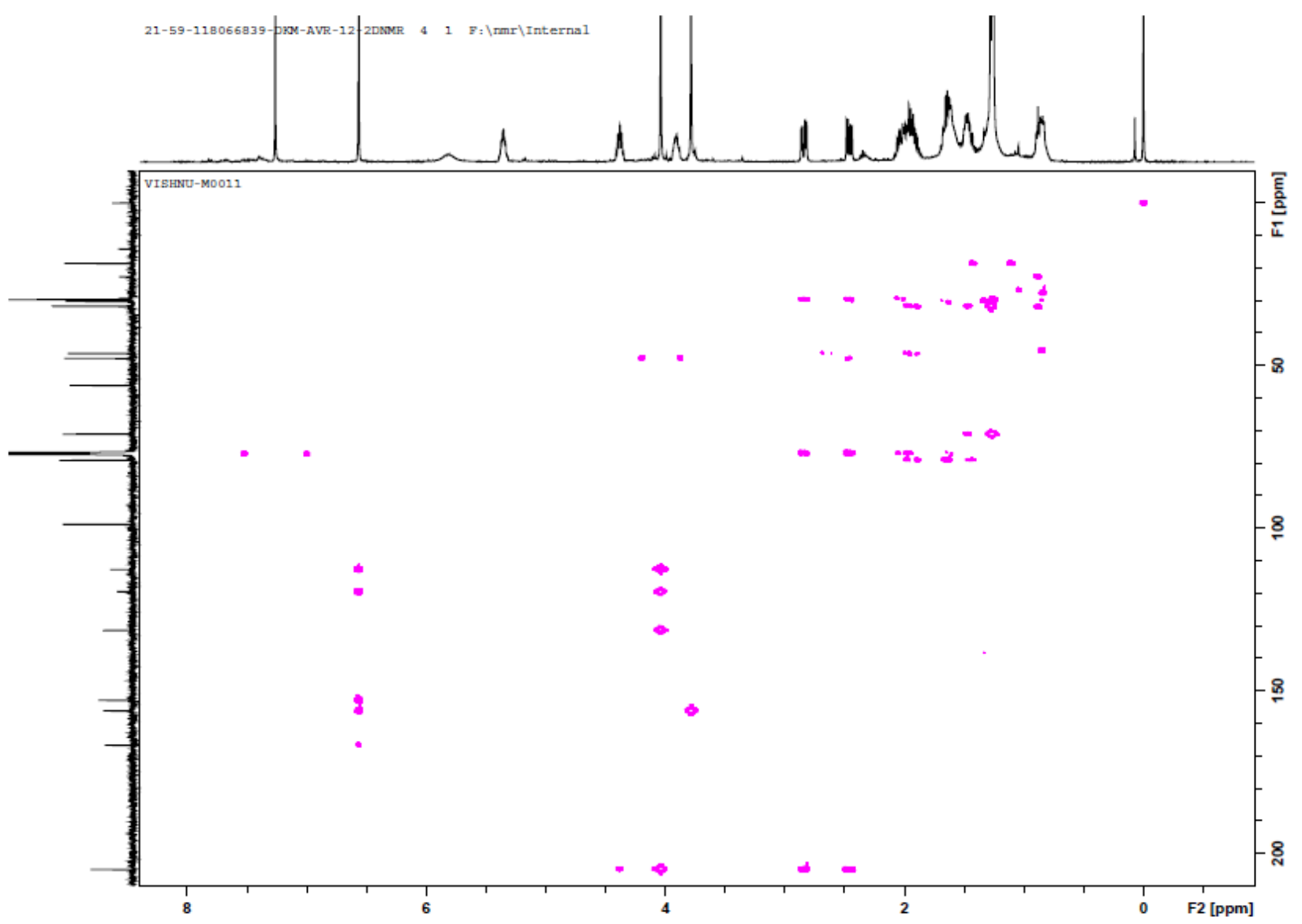

${ }^{13} \mathrm{C}-{ }^{1} \mathrm{H}$ HMBC spectrum of compound 5 (400 MHz, $\mathrm{CDCl}_{3}$ )

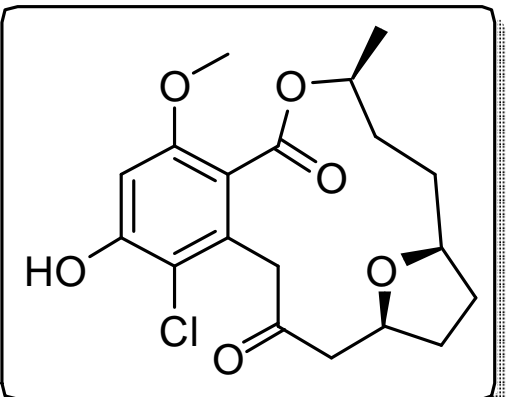




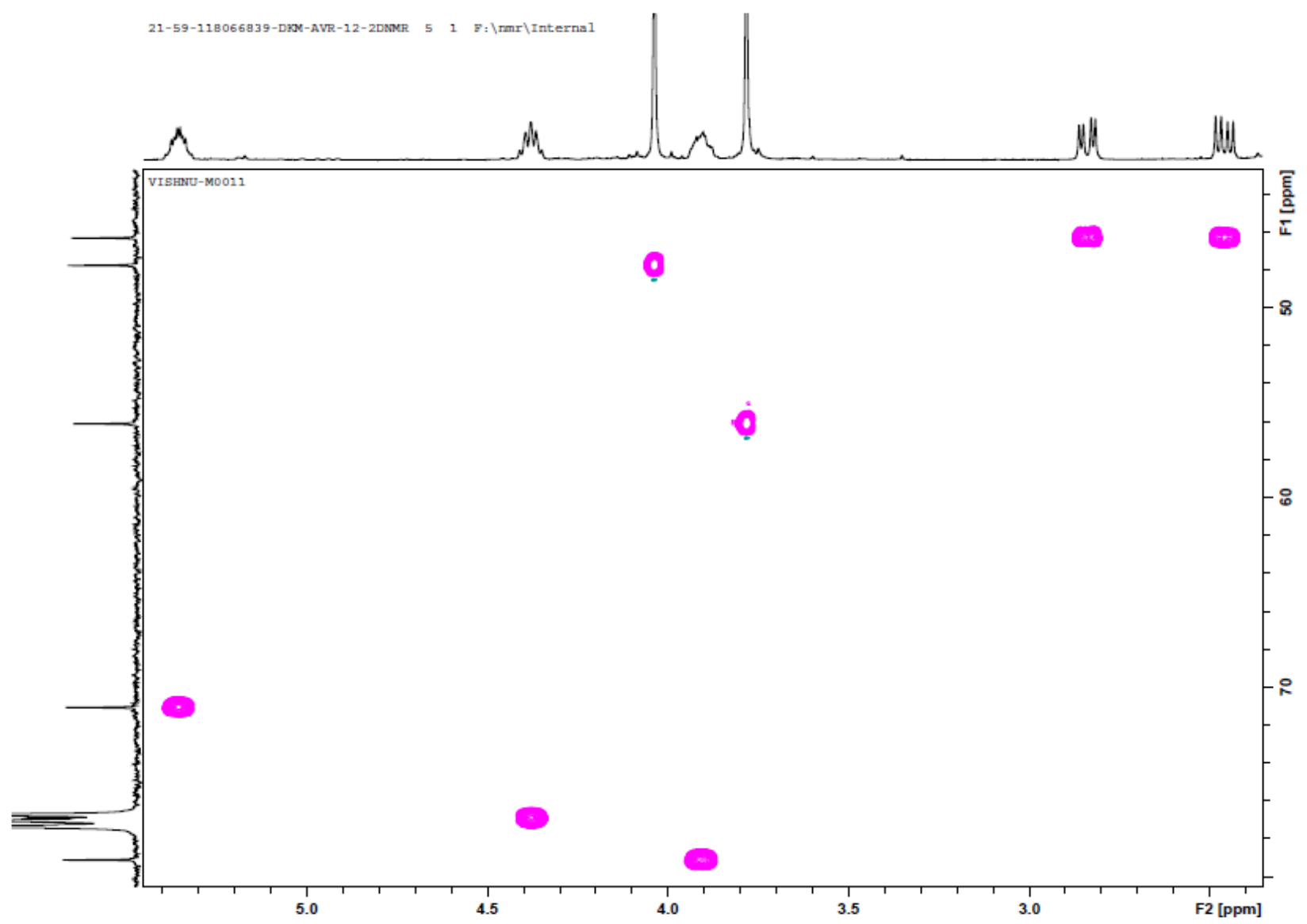

Expanded version of HSQC spectrum of compound $5\left(400 \mathrm{MHz}, \mathrm{CDCl}_{3}\right)$

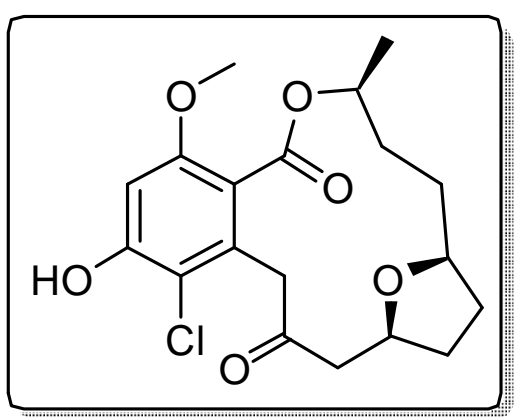




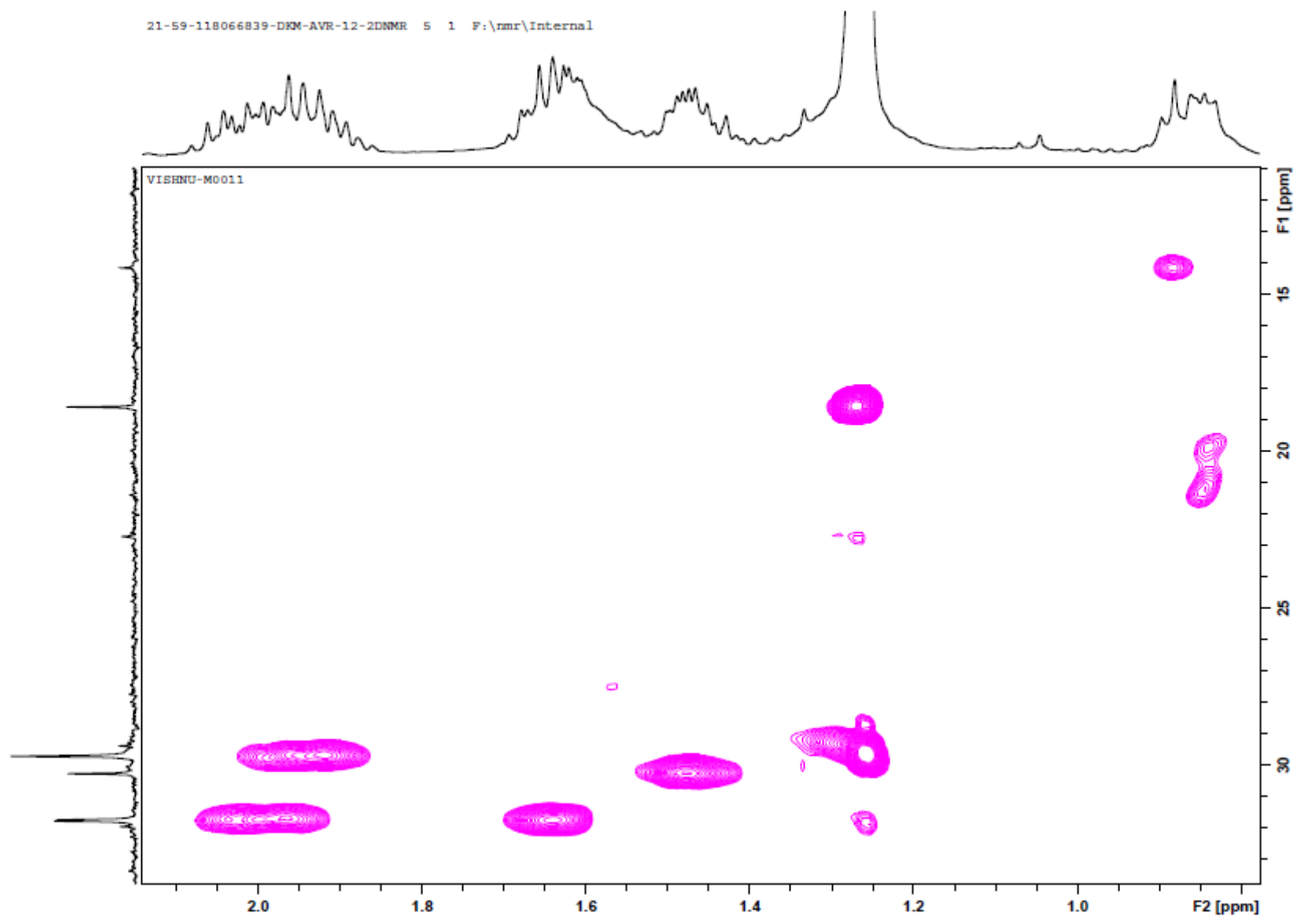

Expanded version of HSQC spectrum of compound $5\left(400 \mathrm{MHz}, \mathrm{CDCl}_{3}\right)$

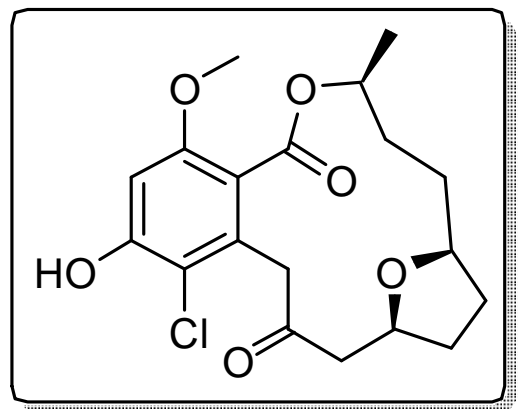




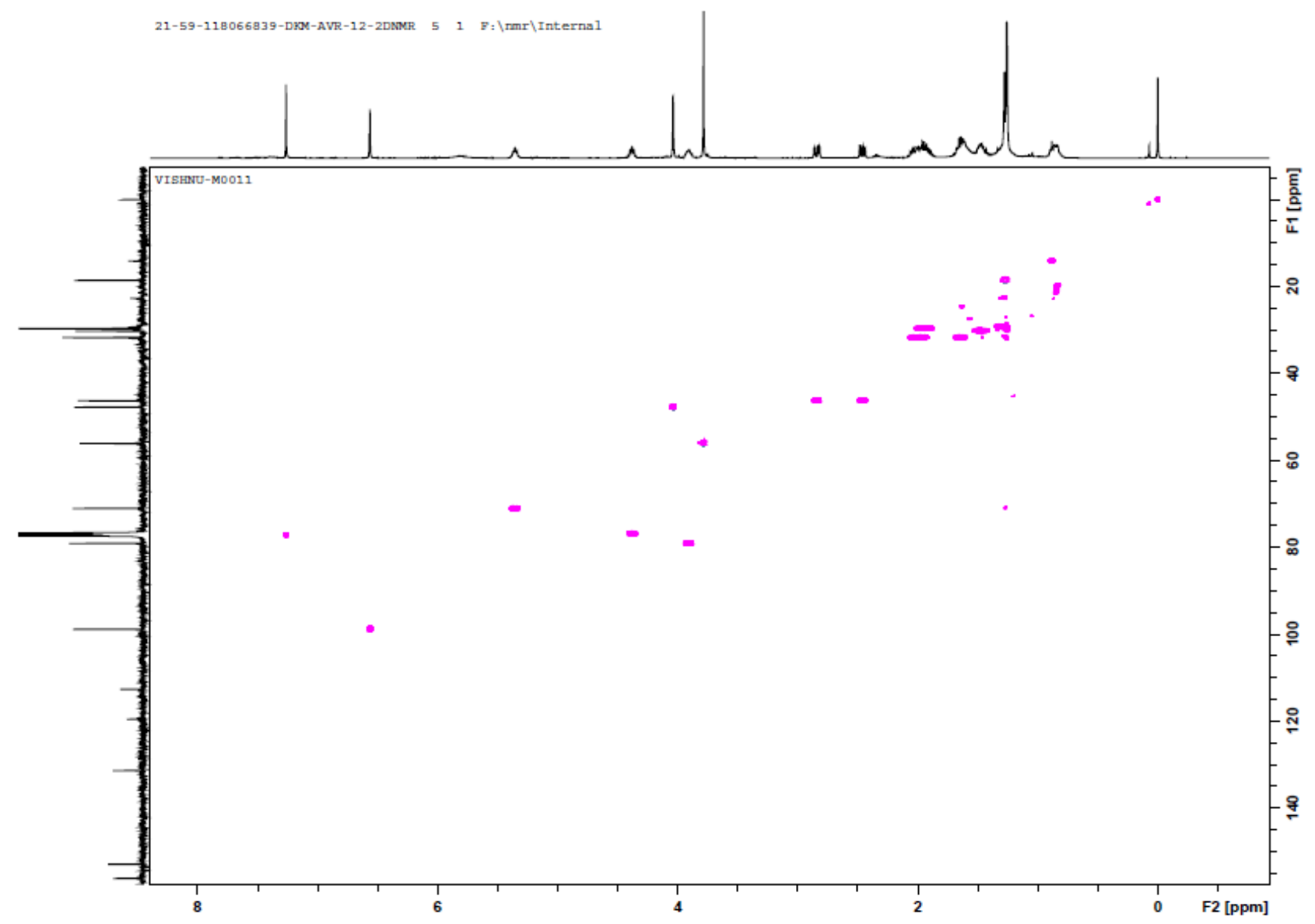

HSQC spectrum of compound $5\left(400 \mathrm{MHz}, \mathrm{CDCl}_{3}\right)$

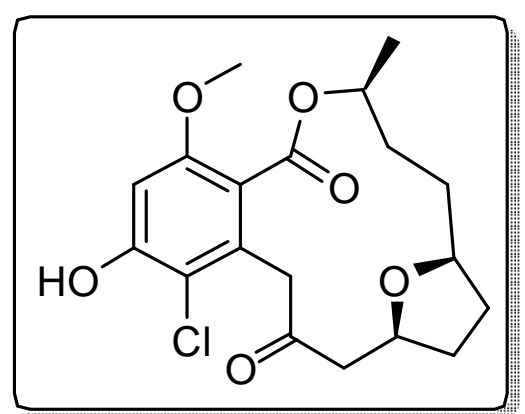




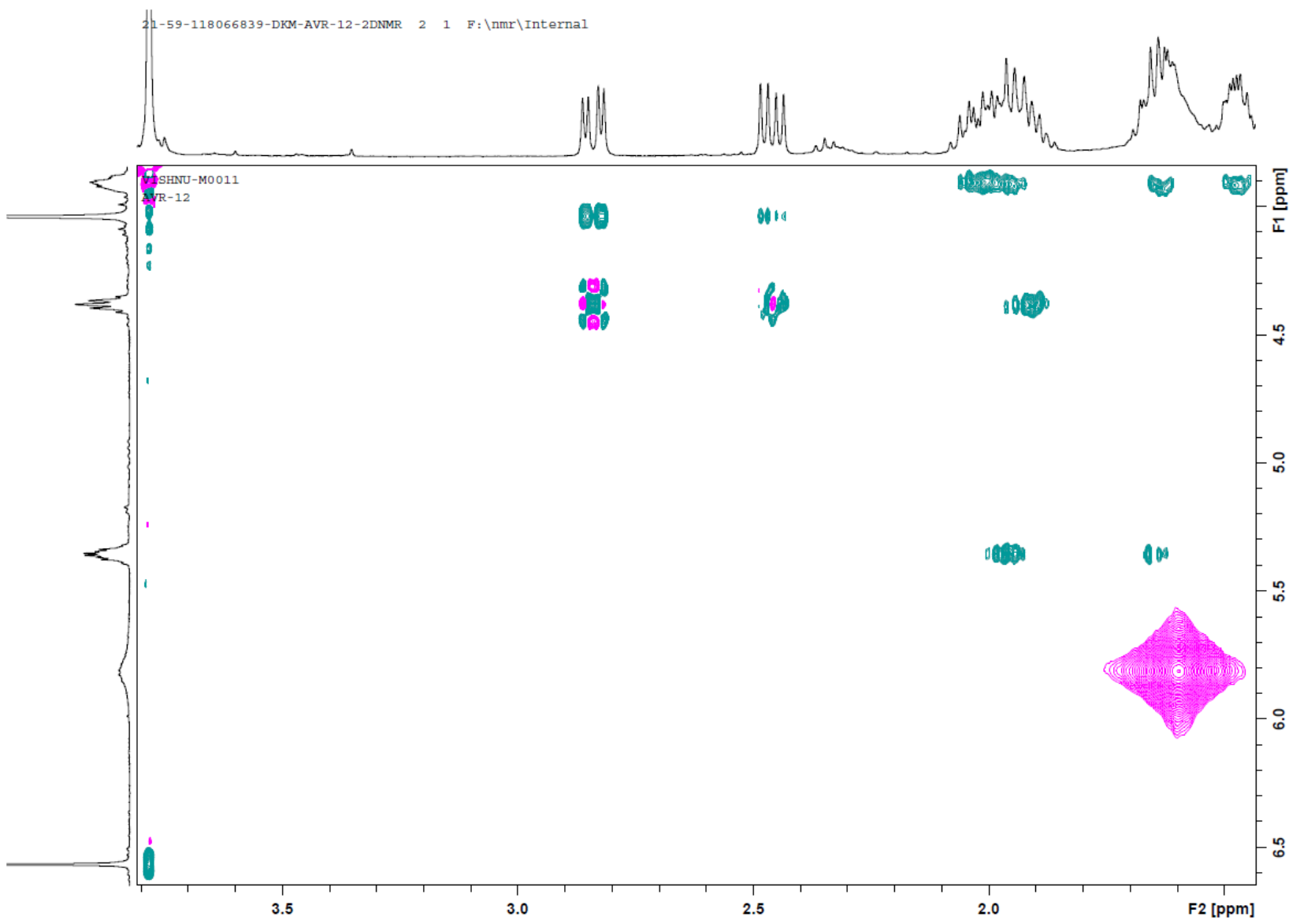

Expanded version of NOESY spectrum of compound $5\left(400 \mathrm{MHz}, \mathrm{CDCl}_{3}\right)$

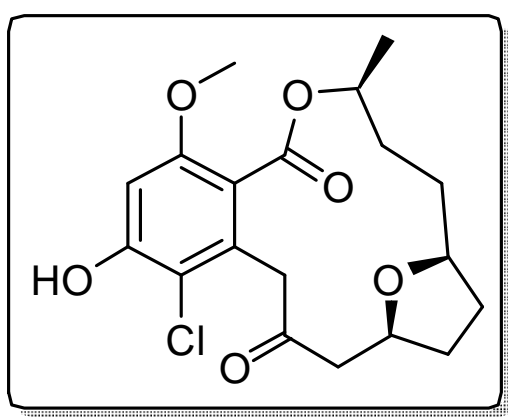




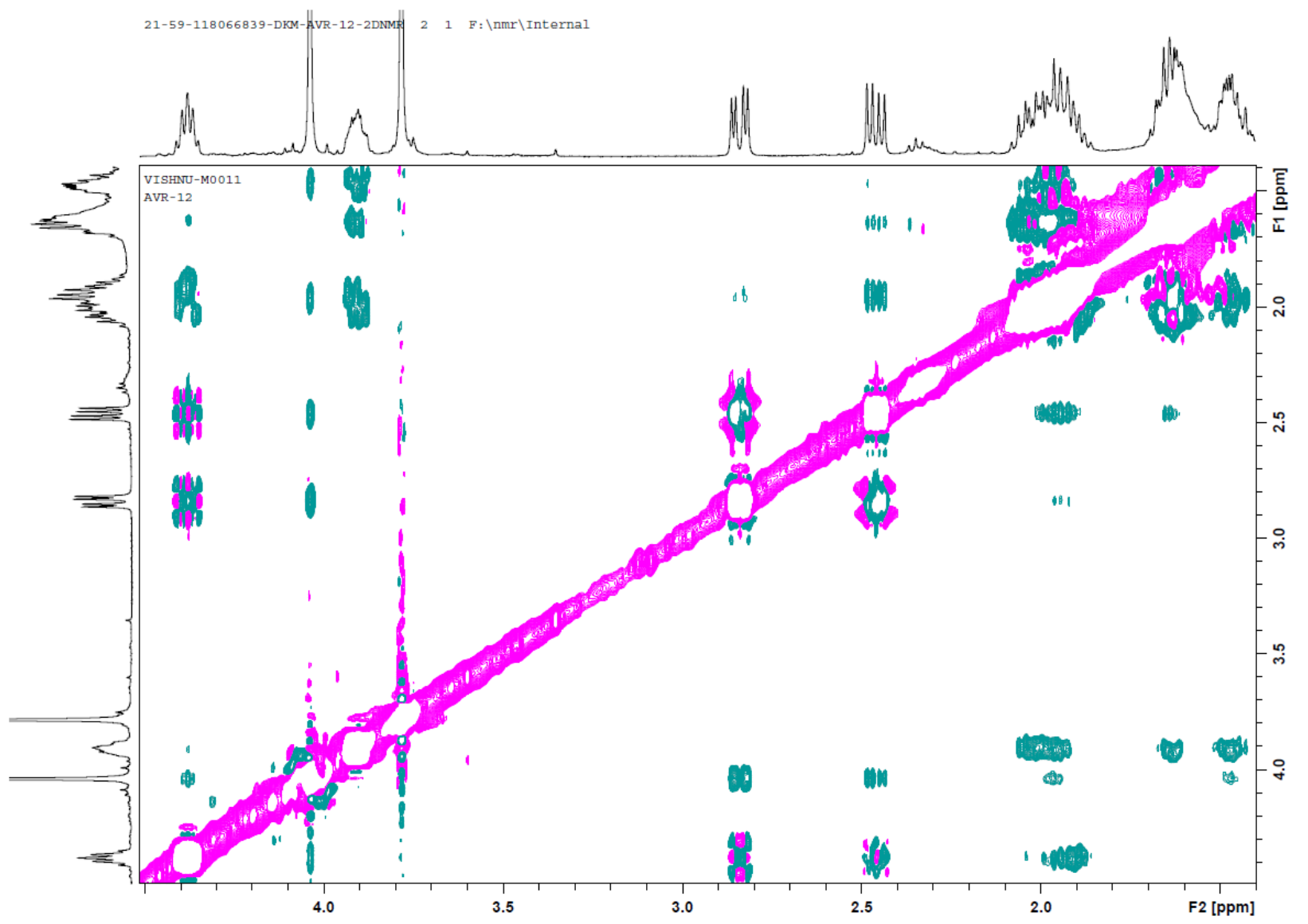

Expanded version of NOESY spectrum of compound $5\left(400 \mathrm{MHz}, \mathrm{CDCl}_{3}\right)$

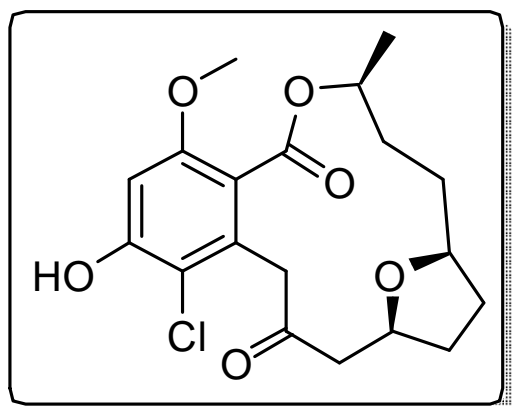




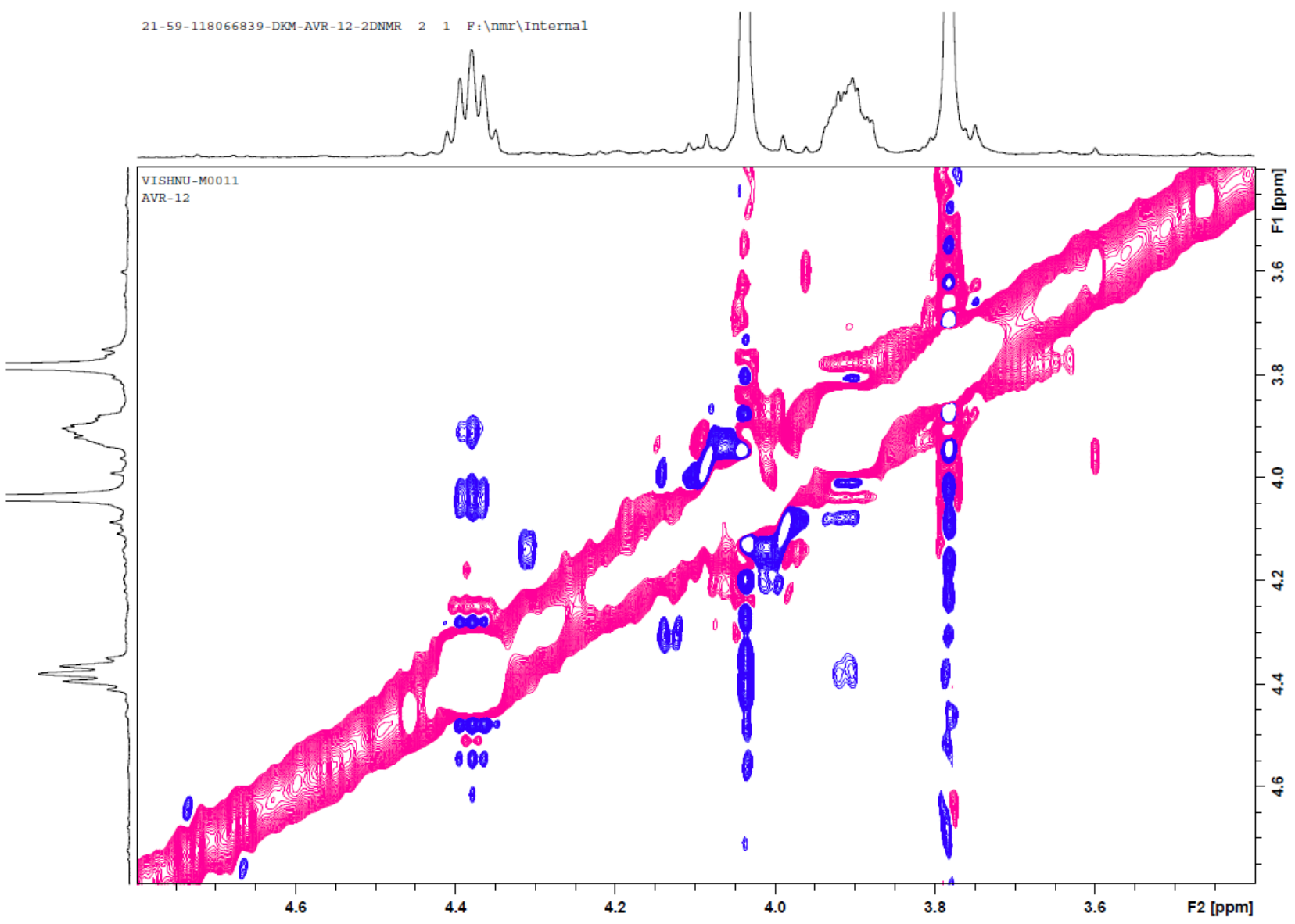

Expanded version of NOESY spectrum of compound $5\left(400 \mathrm{MHz}, \mathrm{CDCl}_{3}\right)$

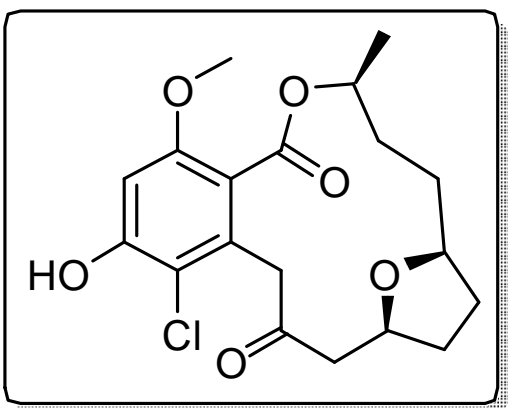




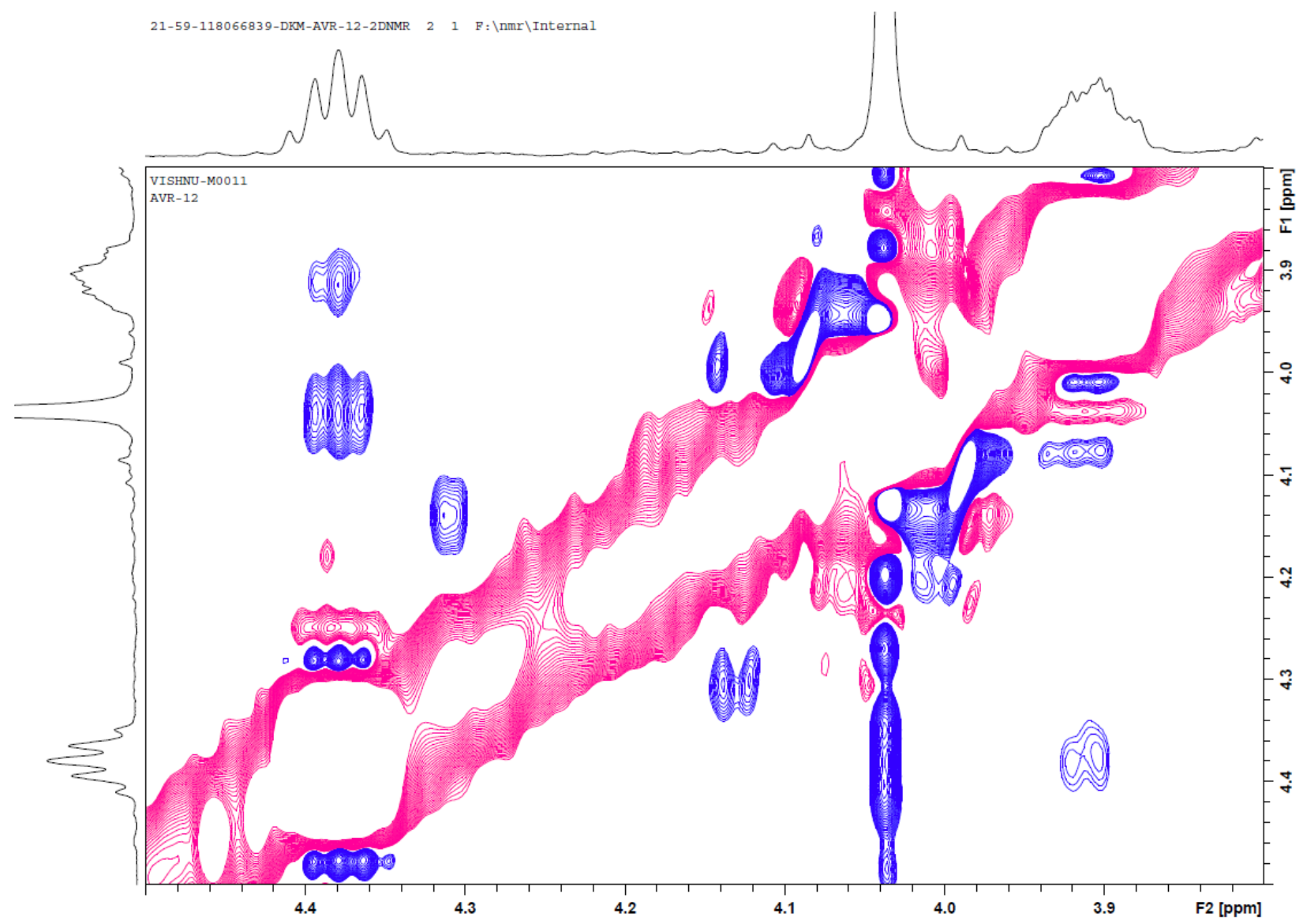

Expanded version of NOESY spectrum of compound $5\left(400 \mathrm{MHz}, \mathrm{CDCl}_{3}\right)$

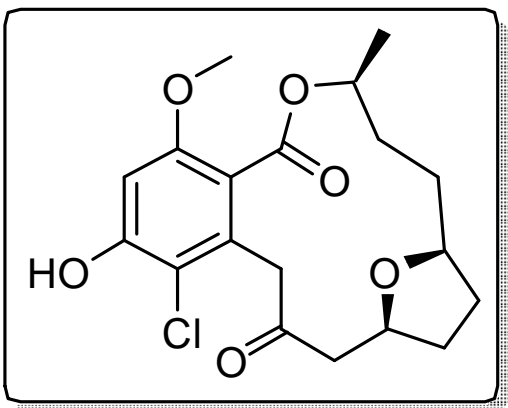




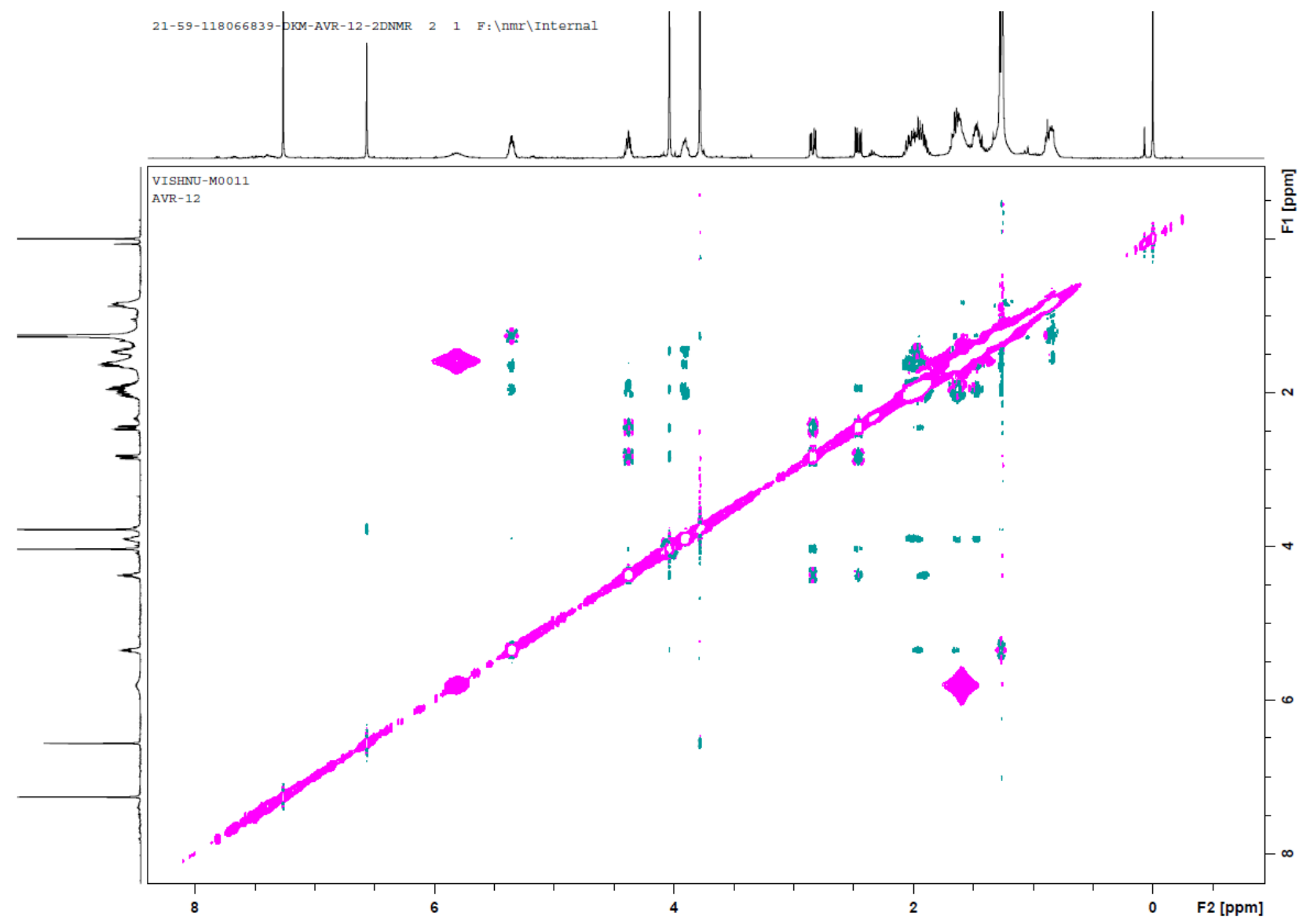

NOESY spectrum of compound $5\left(400 \mathrm{MHz}, \mathrm{CDCl}_{3}\right)$

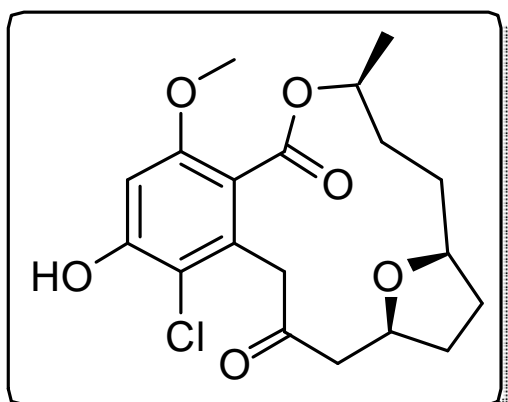




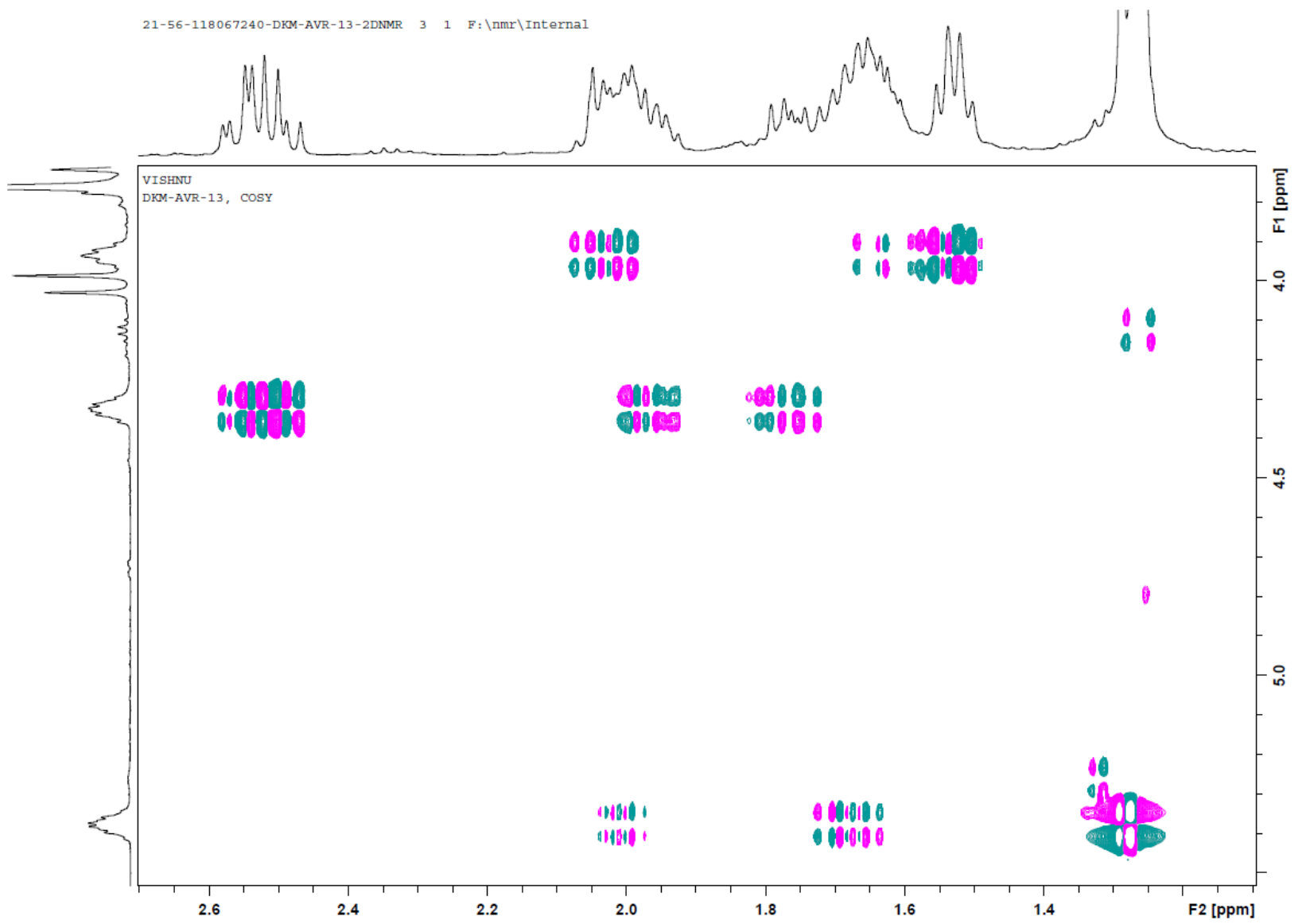

Exapnded version of the DQF-COSY Spectrum of $4\left(400 \mathrm{MHz}, \mathrm{CDCl}_{3}\right)$

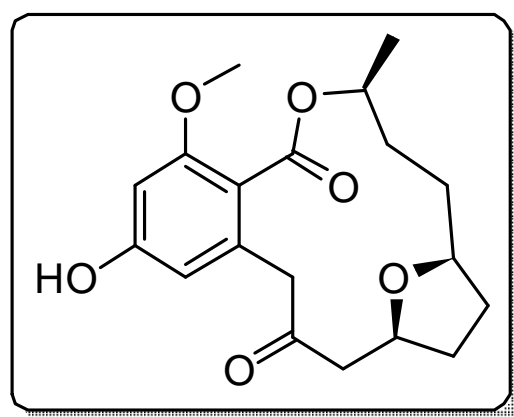




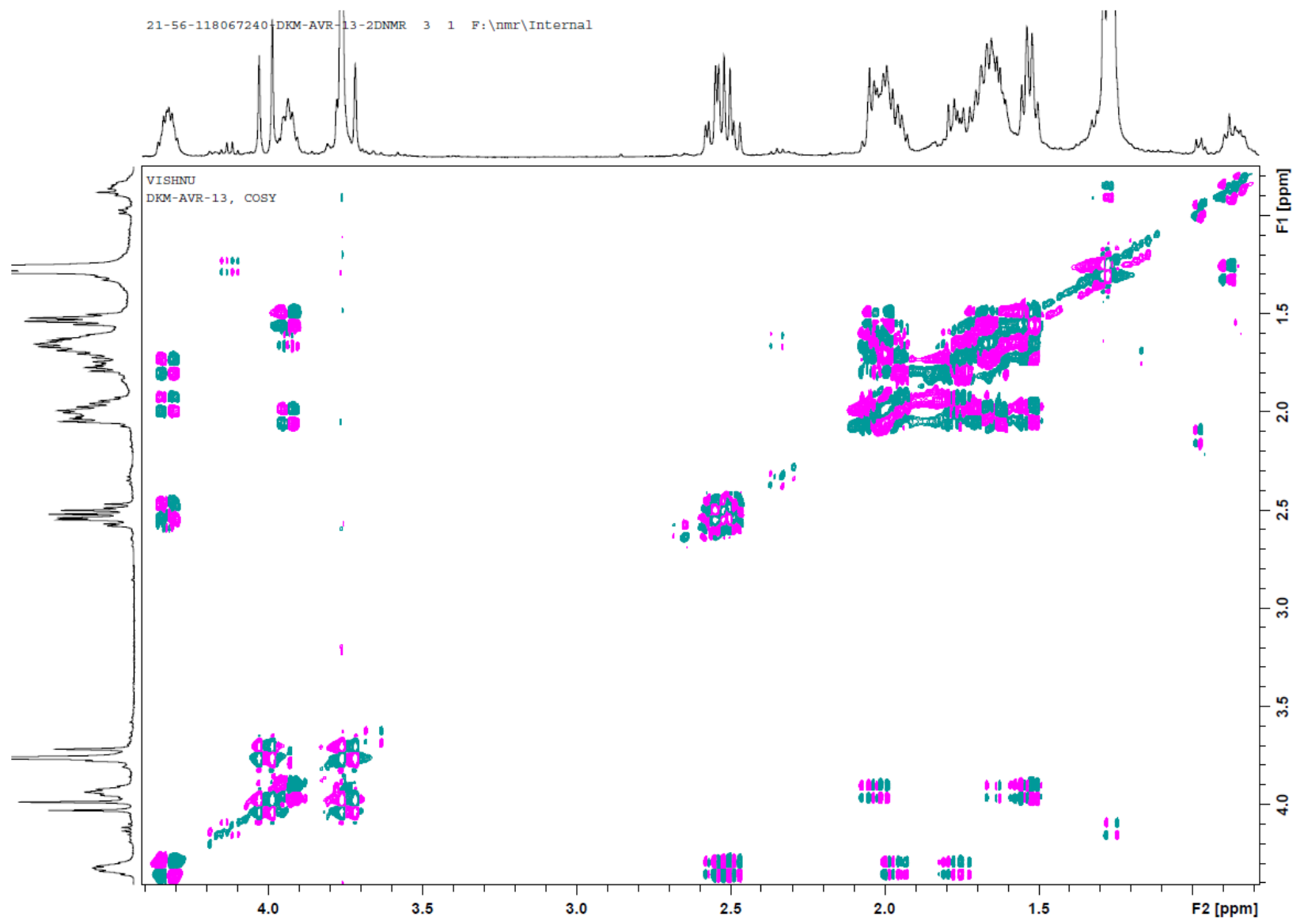

Exapnded version of the DQF-COSY Spectrum of $4\left(400 \mathrm{MHz}, \mathrm{CDCl}_{3}\right)$

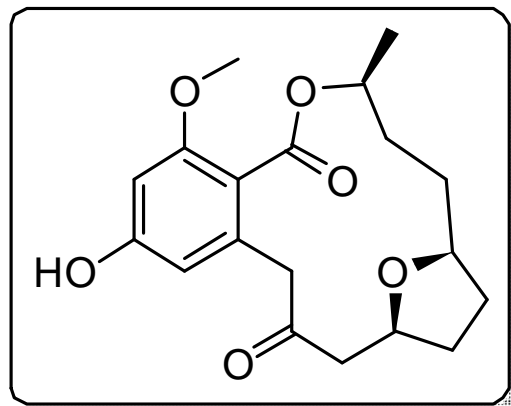




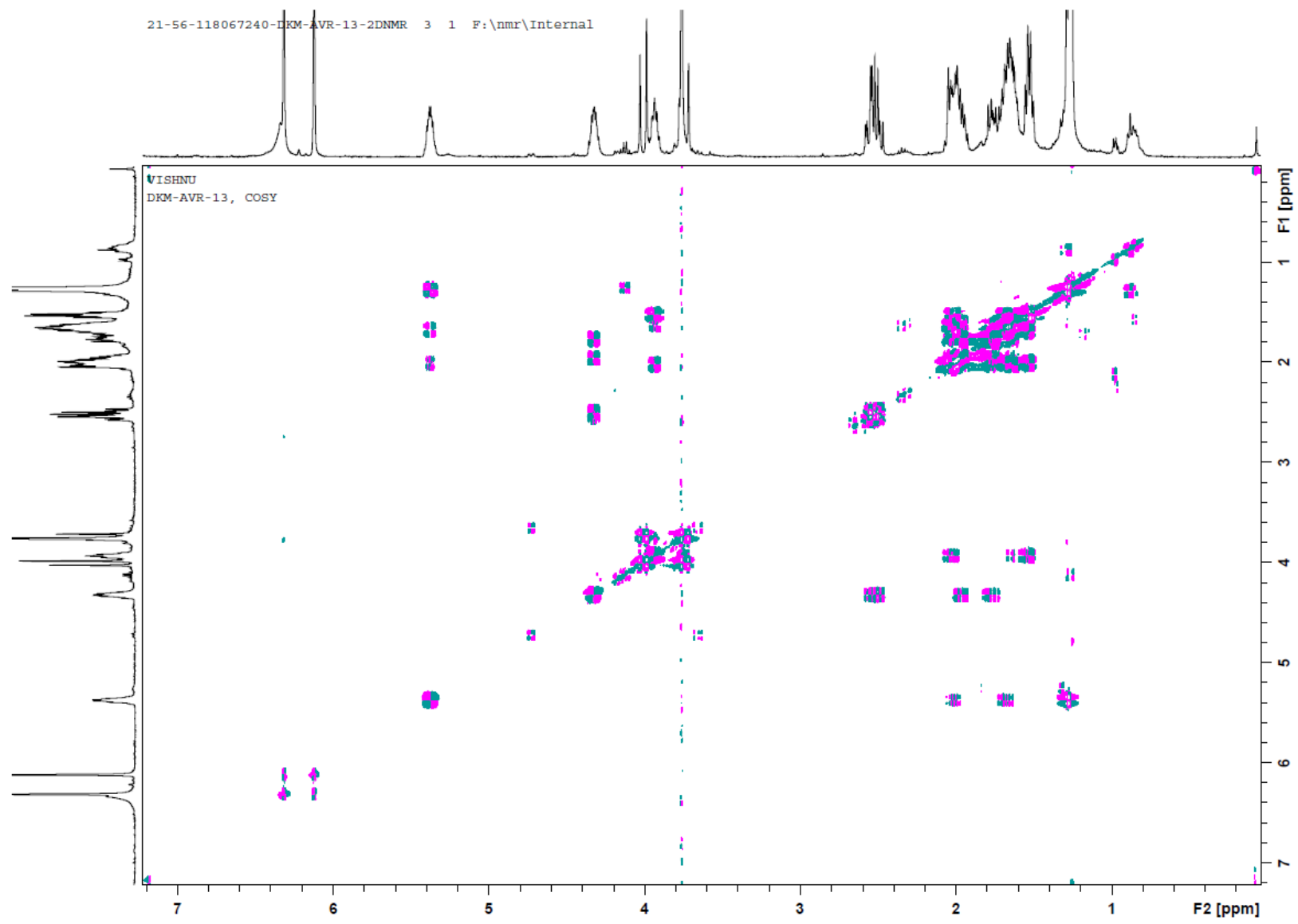

DQF-COSY Spectrum of $4\left(400 \mathrm{MHz}, \mathrm{CDCl}_{3}\right)$

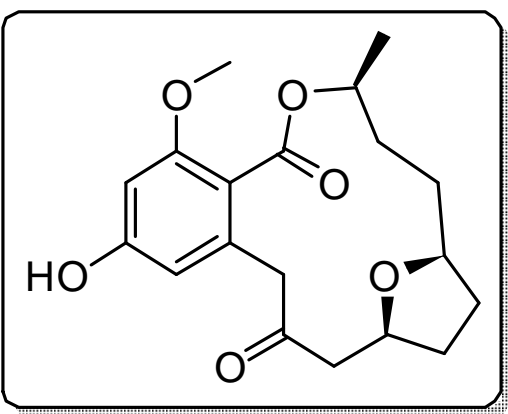


21-56-118067240-DKM-AVR-13-2DNMR $44 \quad 1 \quad \mathrm{~F}: \backslash \mathrm{nmr} \backslash$ Internal

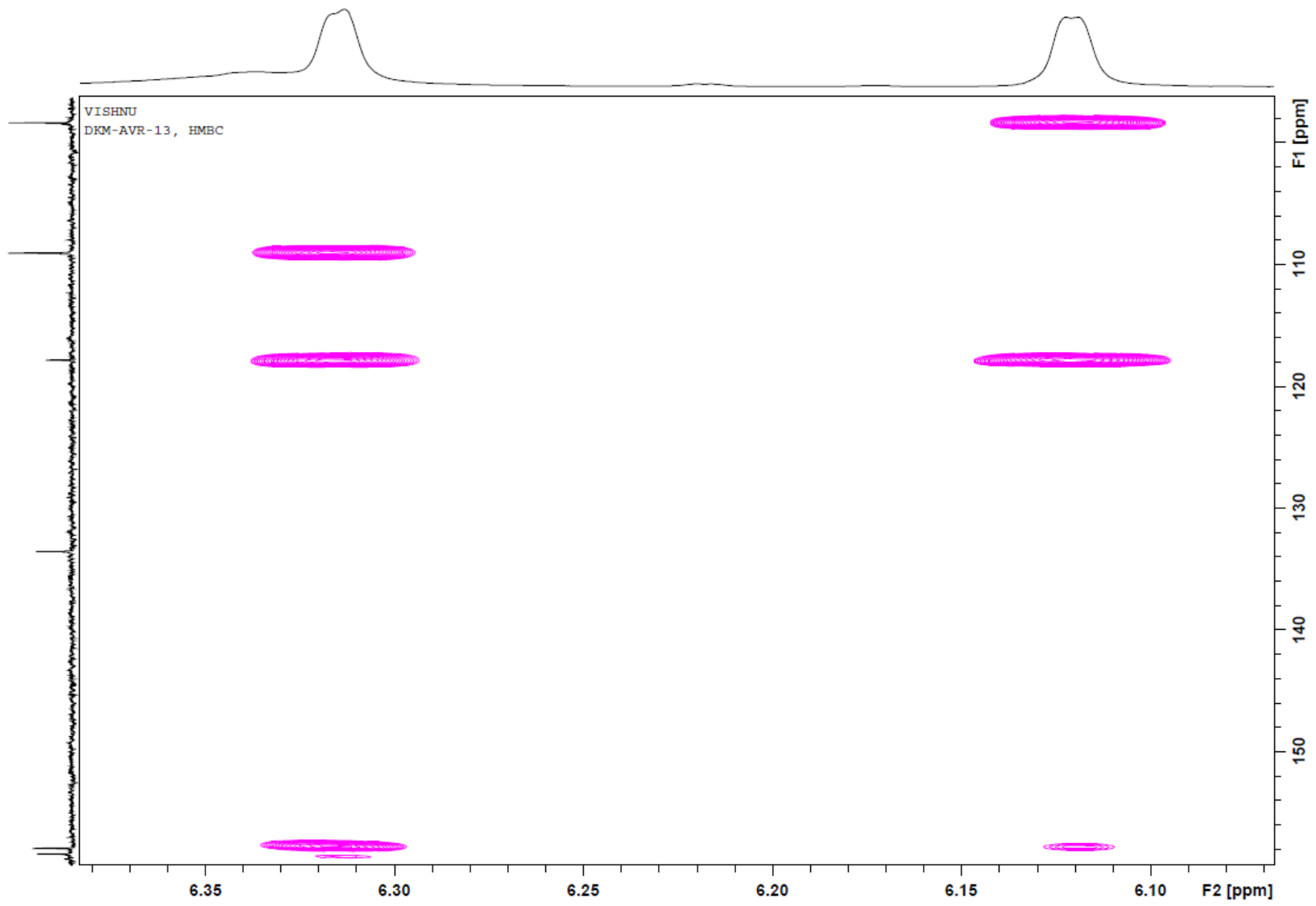

Expanded version of ${ }^{13} \mathrm{C}-{ }^{1} \mathrm{H}$ HMBC spectrum of compound 4 (400 MHz, $\left.\mathrm{CDCl}_{3}\right)$

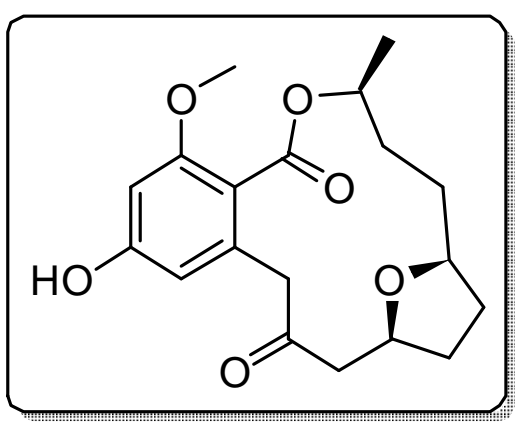




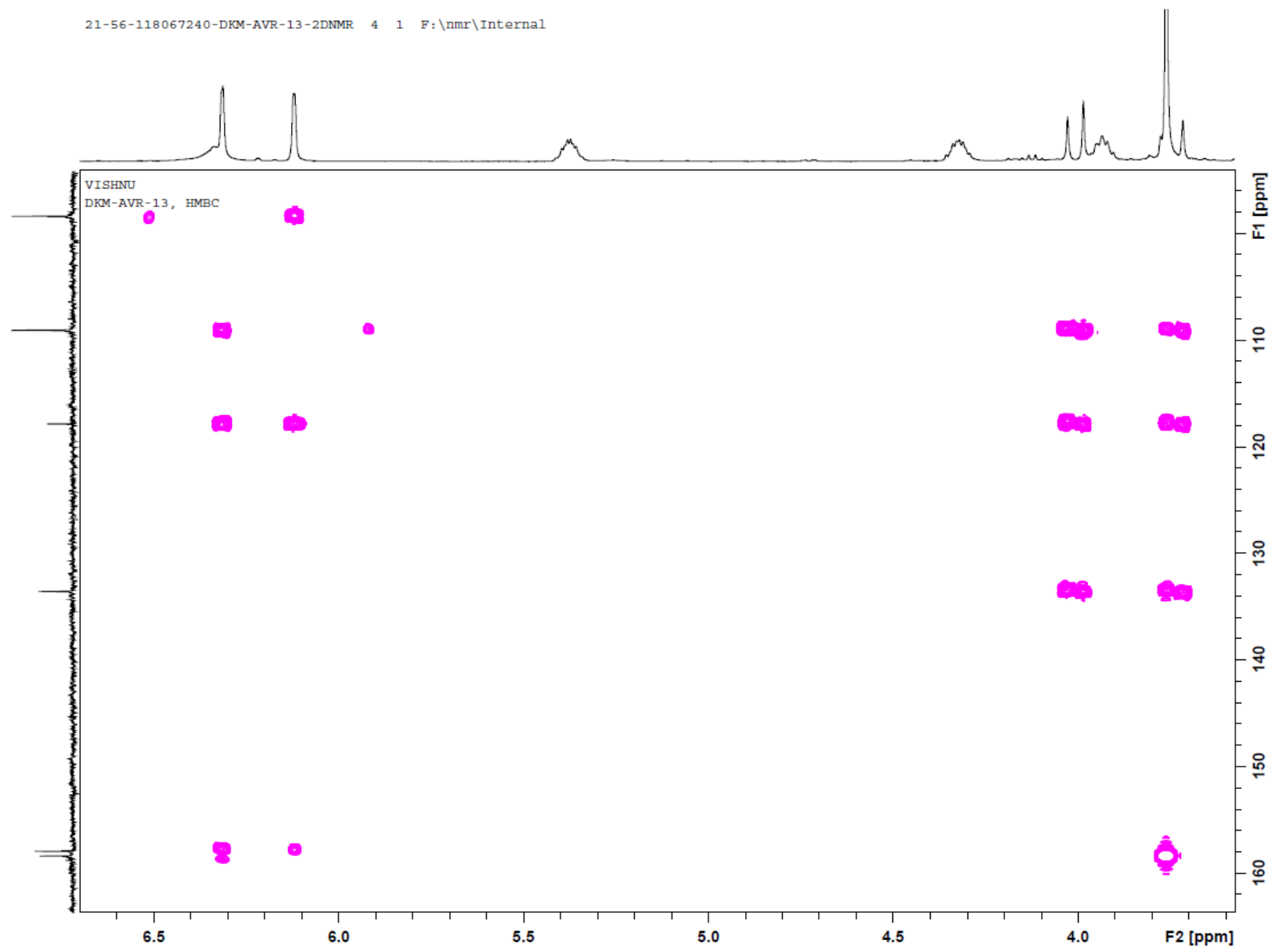

Expanded version of ${ }^{13} \mathrm{C}-{ }^{1} \mathrm{H}$ HMBC spectrum of compound $4\left(400 \mathrm{MHz}, \mathrm{CDCl}_{3}\right)$

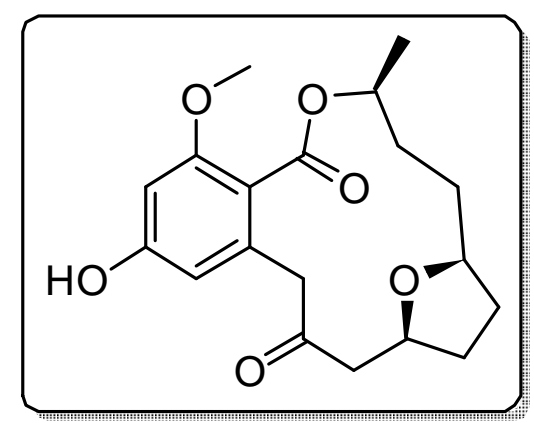




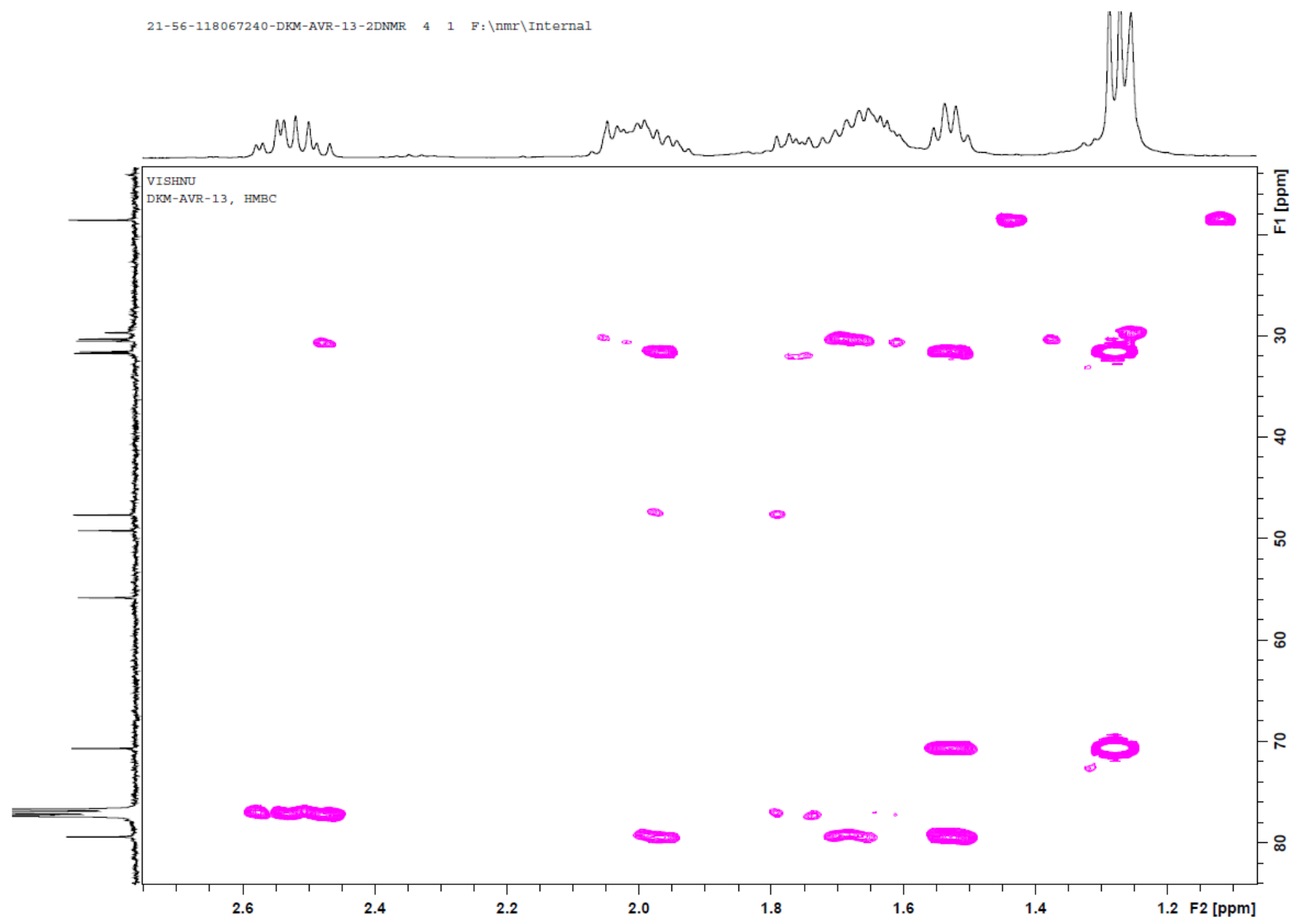

Expanded version of ${ }^{13} \mathrm{C}-{ }_{-}^{1} \mathrm{H}$ HMBC spectrum of compound $4\left(400 \mathrm{MHz}, \mathrm{CDCl}_{3}\right)$

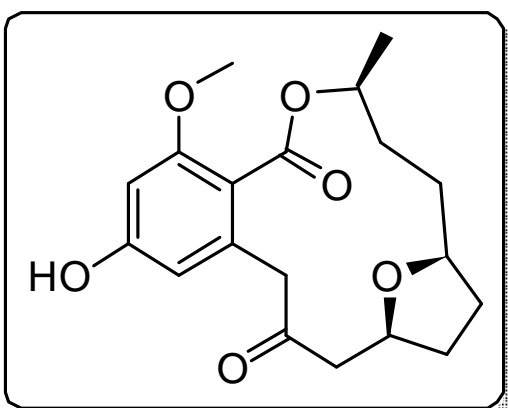




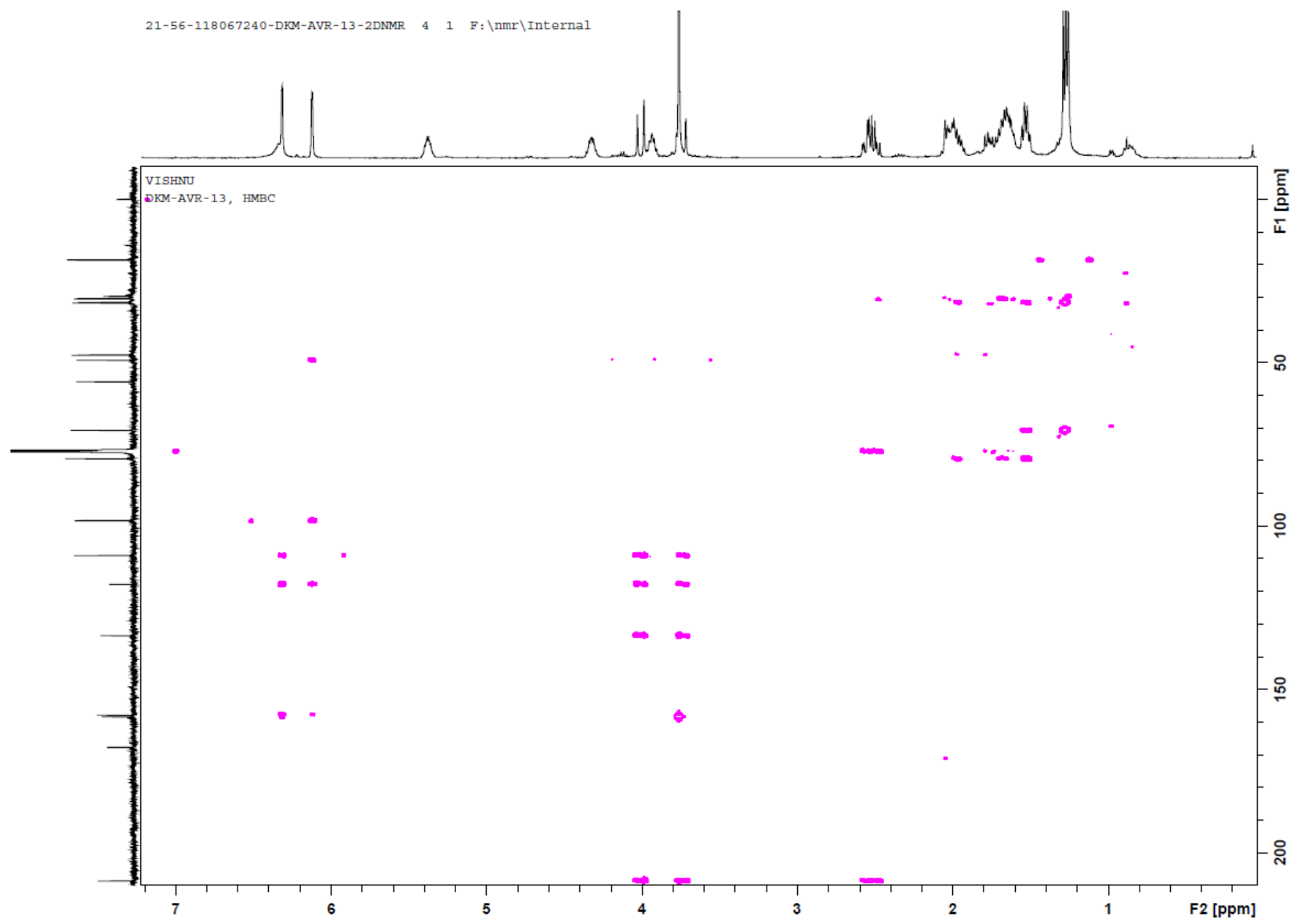

${ }^{13} \mathrm{C}-{ }^{1} \mathrm{H}$ HMBC spectrum of compound 4 (400 $\mathrm{MHz}, \mathrm{CDCl}_{3}$ )

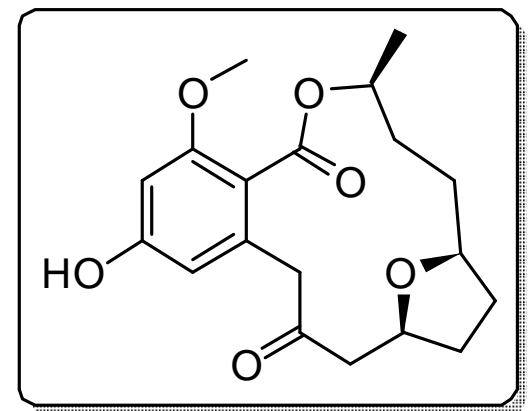




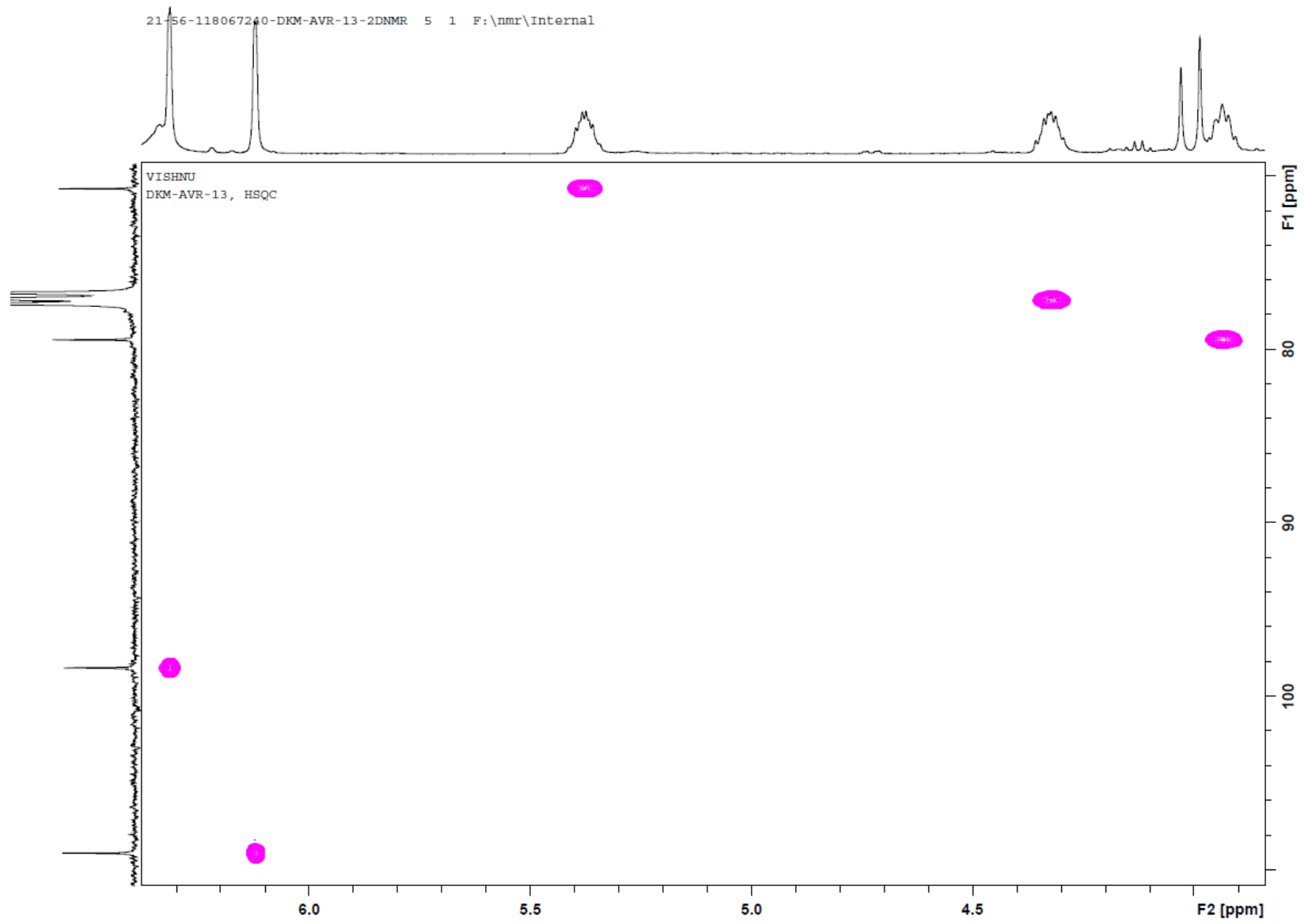

Expanded version of HSQC spectrum of compound 4 (400 MHz, $\mathrm{CDCl}_{3}$ )

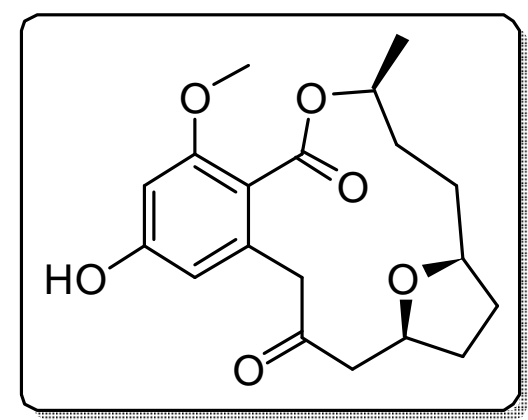




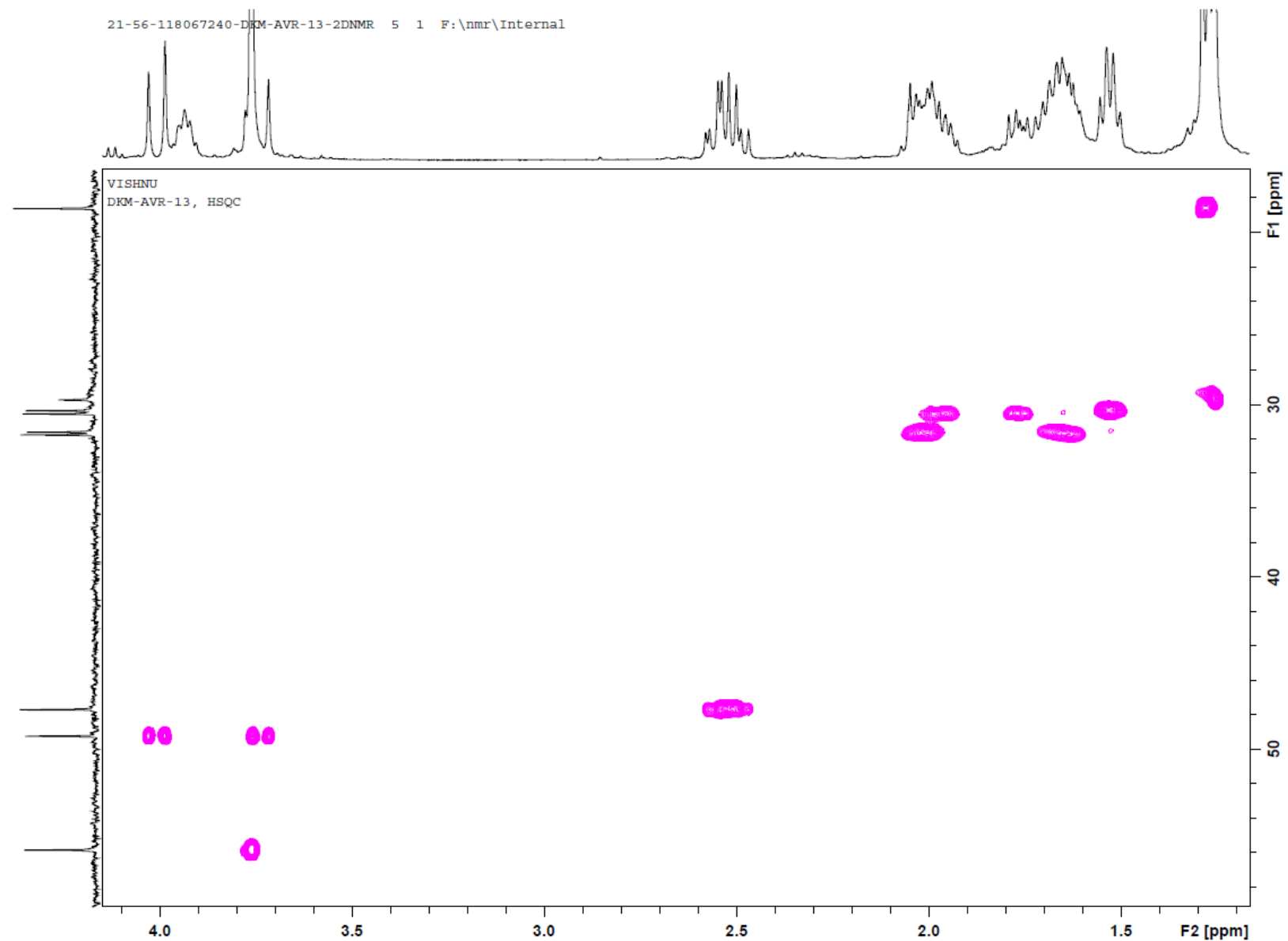

Expanded version of HSQC spectrum of compound $4\left(400 \mathrm{MHz}, \mathrm{CDCl}_{3}\right)$

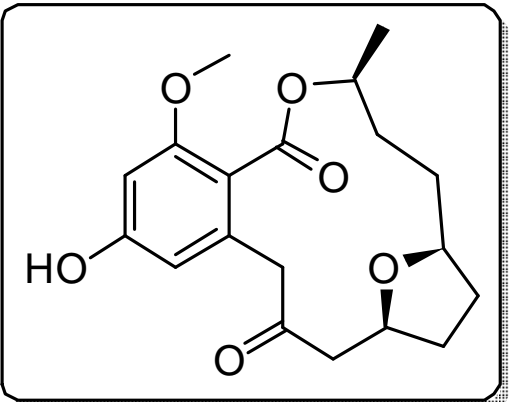




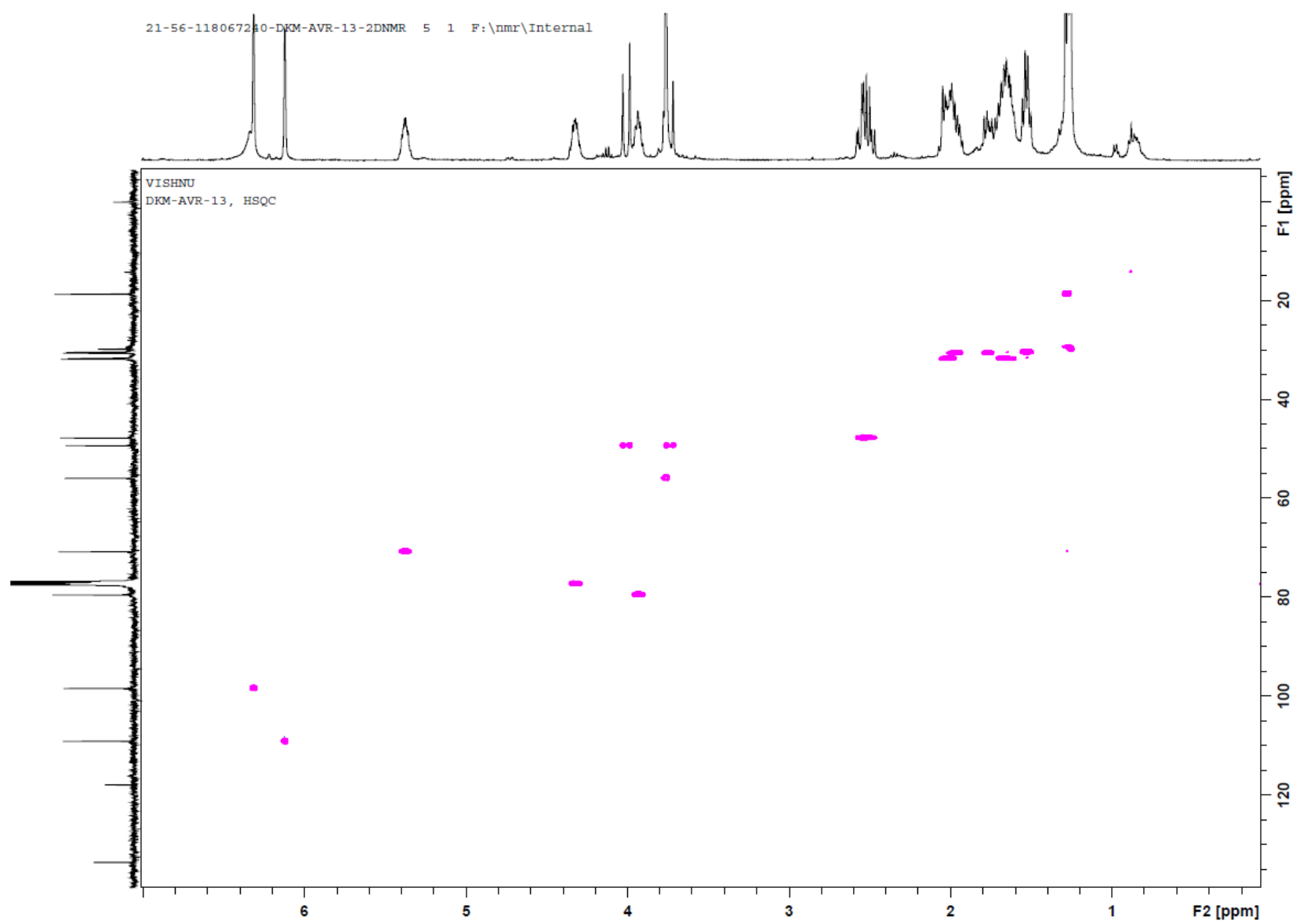

HSQC spectrum of compound $4\left(400 \mathrm{MHz}, \mathrm{CDCl}_{3}\right)$

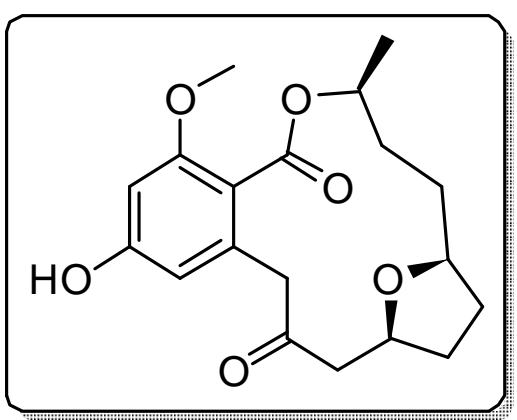




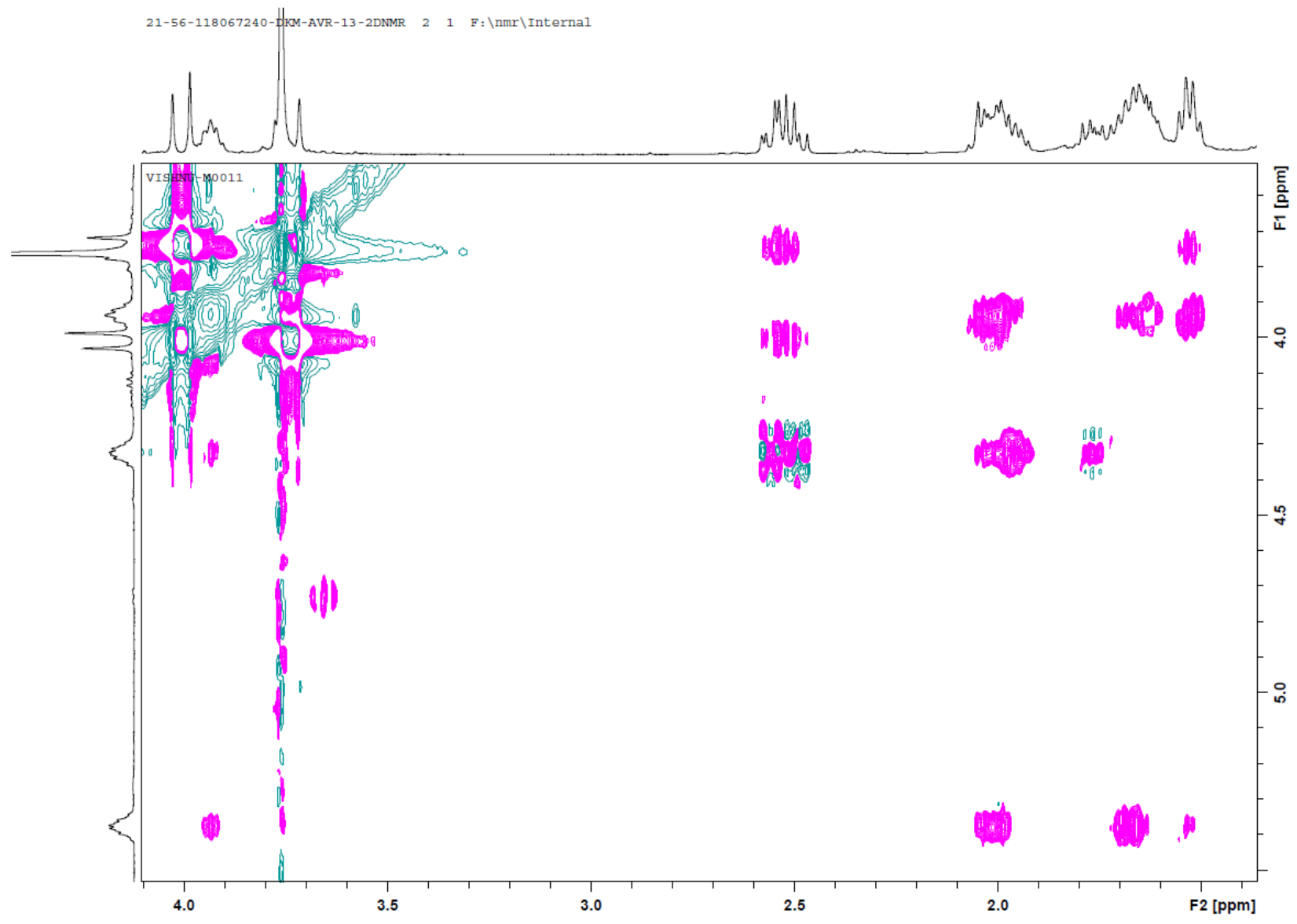

Expanded version of NOESY spectrum of compound $4\left(400 \mathrm{MHz}, \mathrm{CDCl}_{3}\right)$

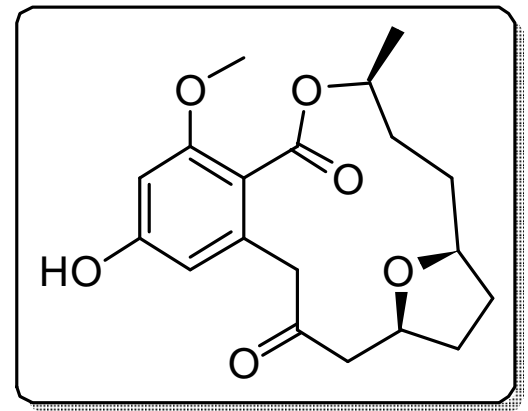




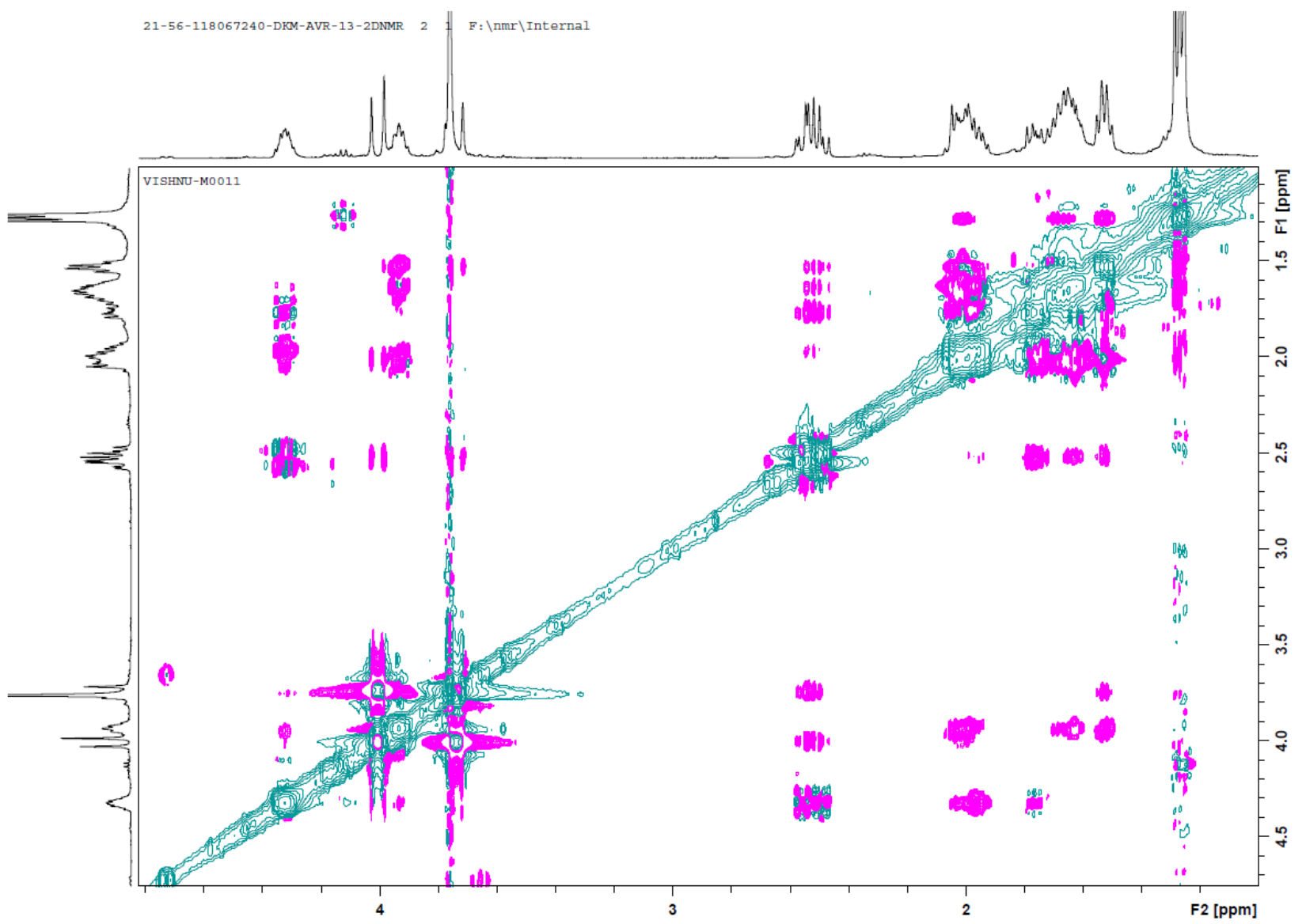

Expanded version of NOESY spectrum of compound $4\left(400 \mathrm{MHz}, \mathrm{CDCl}_{3}\right)$

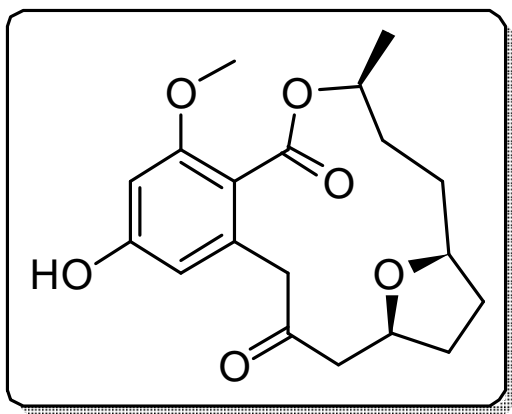




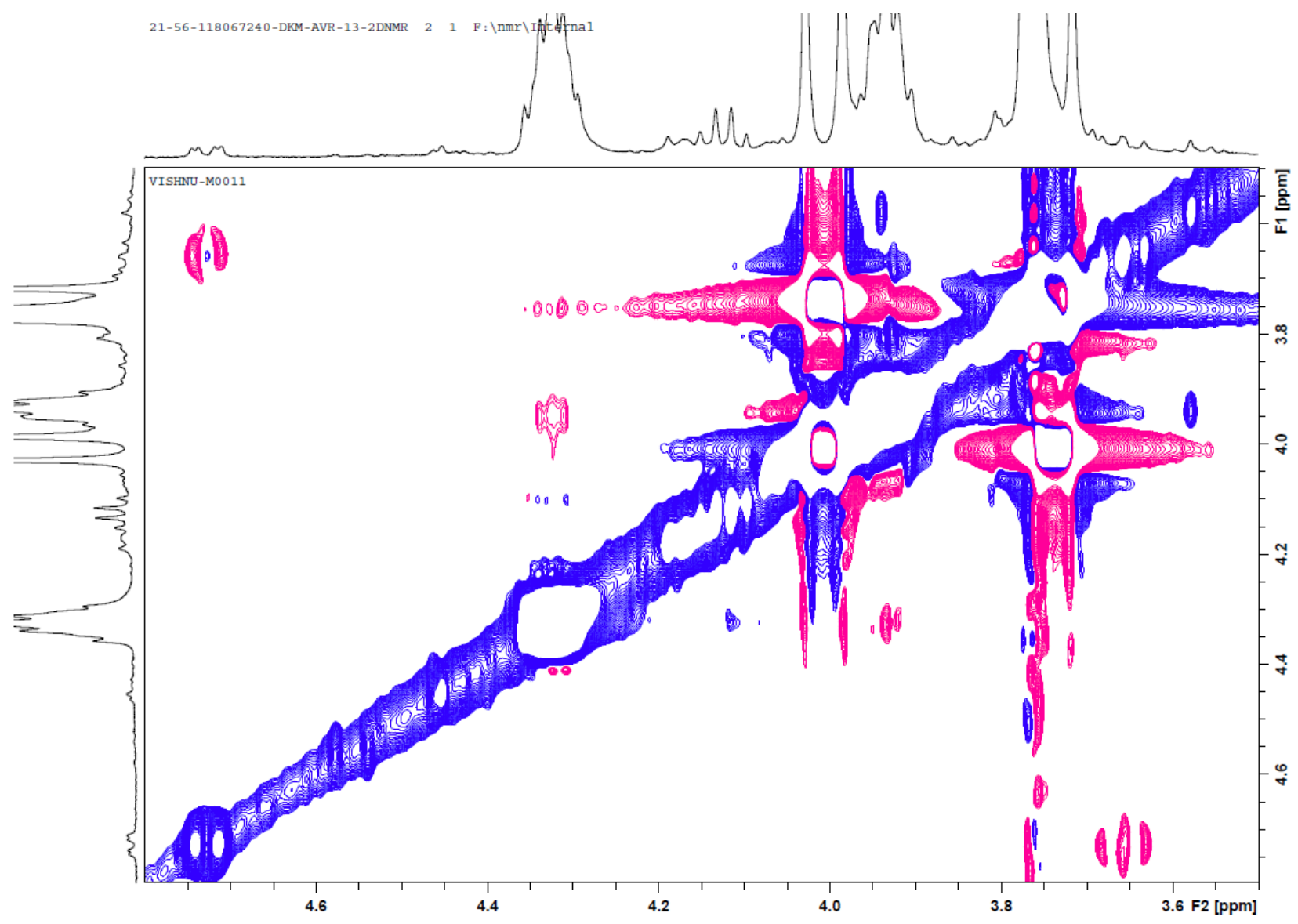

Expanded version of NOESY spectrum of compound $4\left(400 \mathrm{MHz}, \mathrm{CDCl}_{3}\right)$

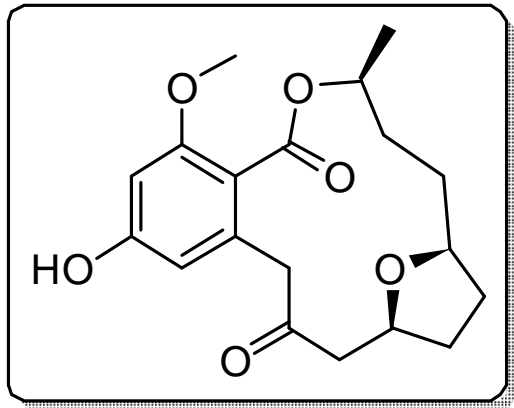




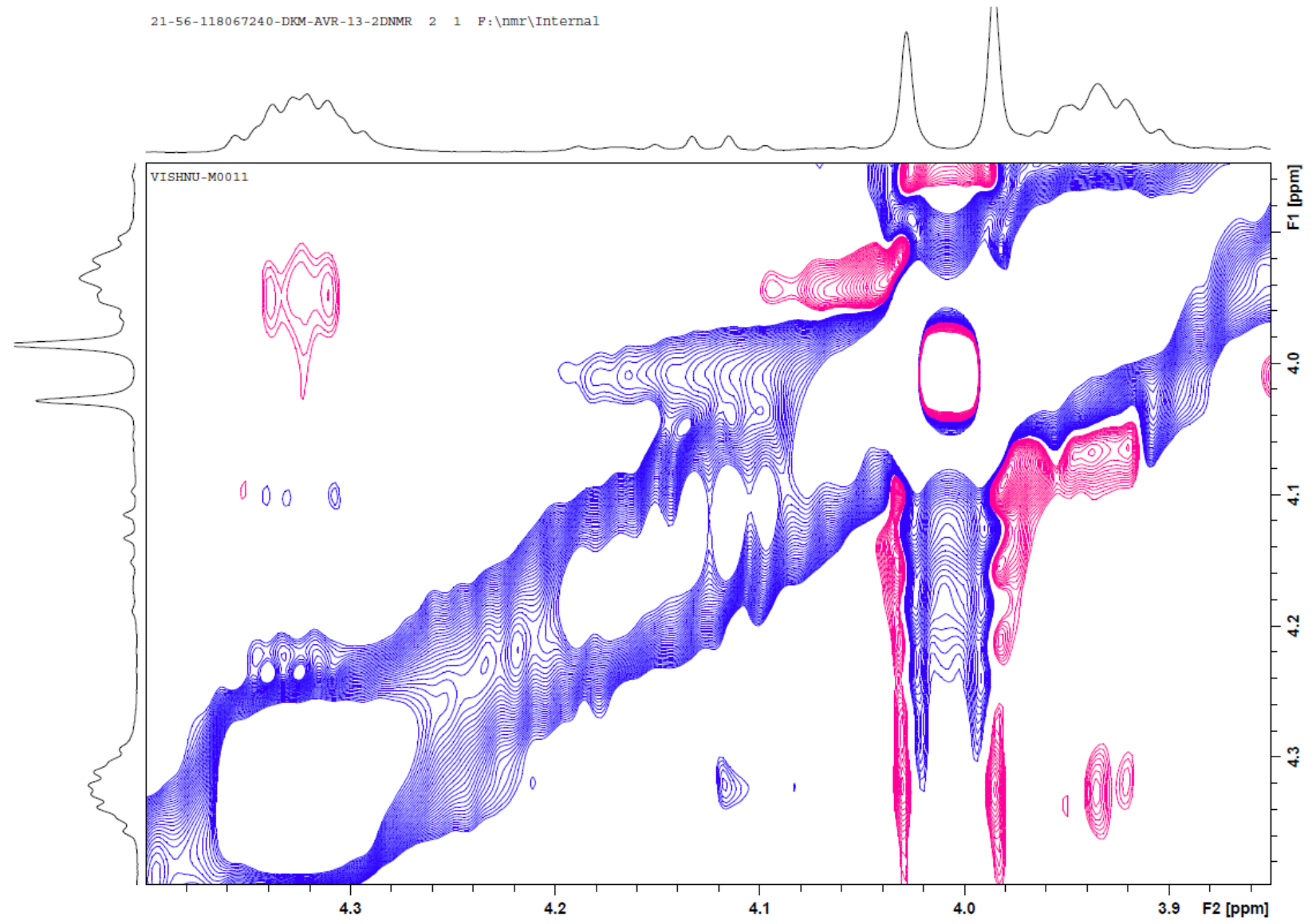

Expanded version of NOESY spectrum of compound $4\left(400 \mathrm{MHz}, \mathrm{CDCl}_{3}\right)$

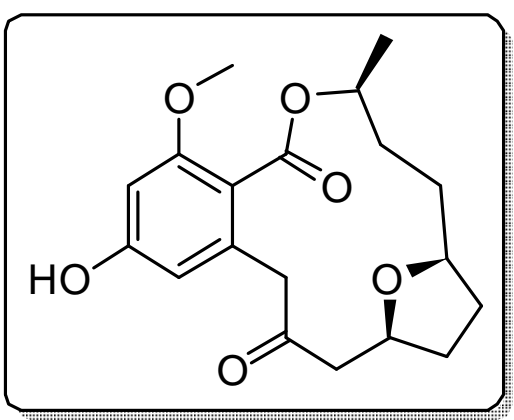




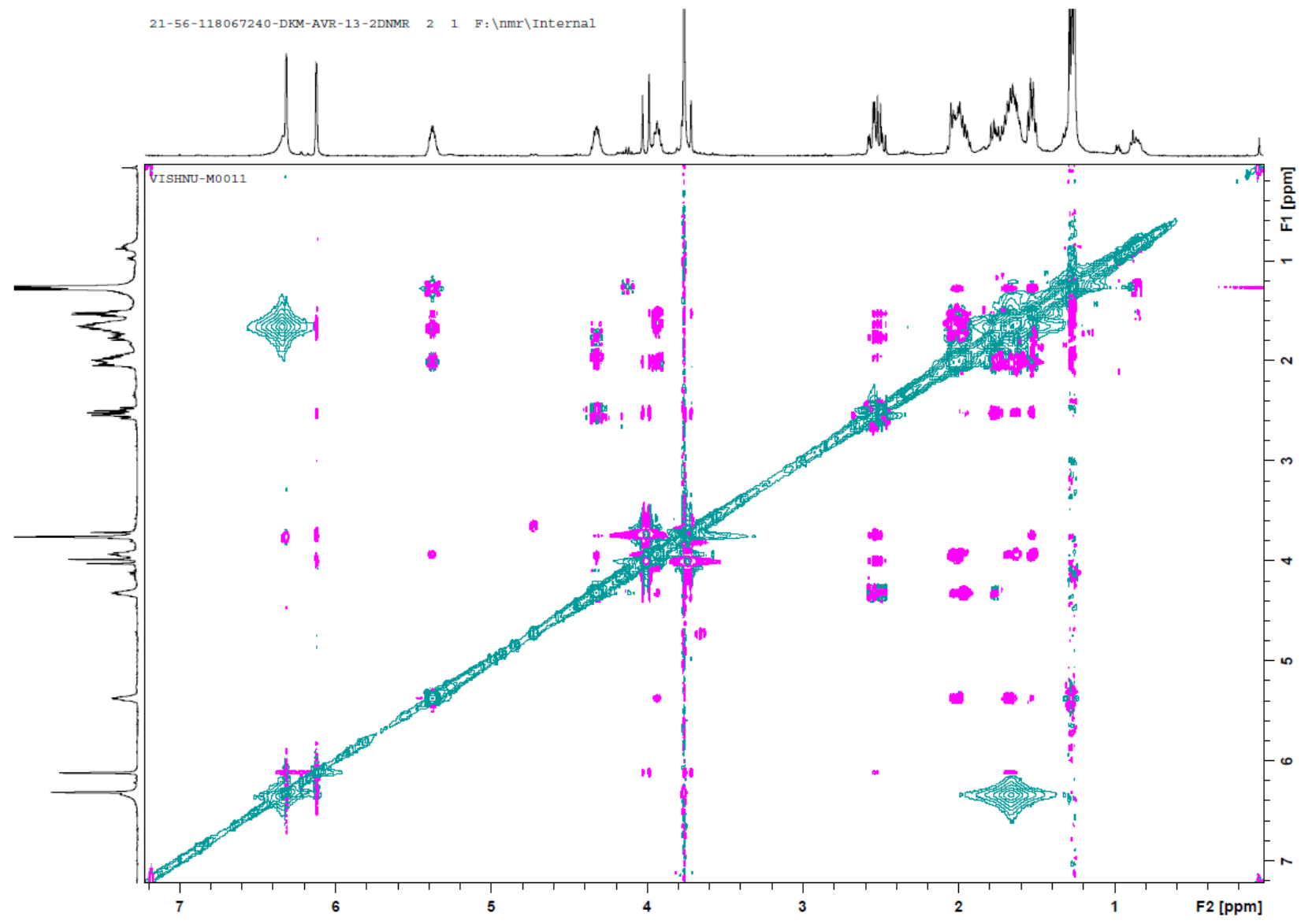

NOESY spectrum of compound $4\left(400 \mathrm{MHz}, \mathrm{CDCl}_{3}\right)$

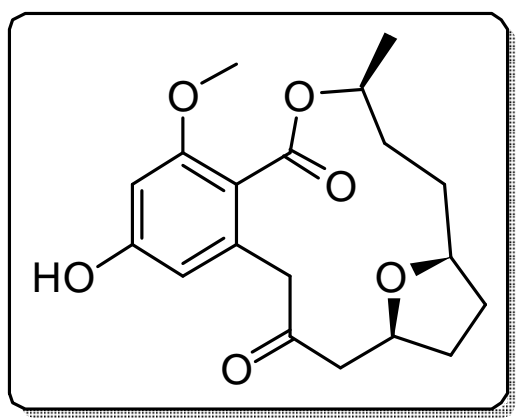

